\title{
Les indicateurs
}

de l'enseignement 



\section{Préface}

C'est toujours un plaisir d'initier un projet pour la première fois et de constater, particulièrement dans le domaine de l'Education, que tant sa qualité que sa fonction et son utilité sont reconnues par ceux qui en font usage.

Mais c'est encore plus satisfaisant, encore plus efficace, si ce projet est pérennisé tant dans sa forme que dans son fond et permet par là, année après année, de se voir prolongé, de gagner encore en valeur et en continuité, et d'avoir les effets escomptés.

C'est bel et bien ce qui advient des Indicateurs de l'Enseignement en Communauté française puisque, après une première publication prometteuse et fort bien réussie, cette deuxième édition, enrichie, mise à jour et attendue avec intérêt, illustre un an plus tard le développement que connaît cet outil d'information et de pilotage majeur pour le système éducatif tout entier.

Avec, entre autres, les évaluations externes des acquis des élèves, les enquêtes et études internationales et la recherche et développement en éducation de manière générale, cette publication de la deuxième édition des indicateurs de l'enseignement en Communauté française trouve pleinement sa place parmi les outils de pilotage nécessaires à tout système éducatif de qualité. Bien plus qu'un simple exercice de présentation et de démonstration statistique, elle éclairera chaque lecteur - qu'il soit usager du système éducatif, parent d'élève, responsable, journaliste ou simplement citoyen - sur le contexte dans lequel évolue l'enseignement de la Communauté française et ses besoins, sur son organisation et son fonctionnement, sur le rôle et la composition du corps enseignant ainsi que sur le suivi statistique des élèves et leur acquisition des compétences et savoirs qui leur sont indispensables au sein de la société moderne de la connaissance.

Ces informations harmonisées s'intègrent précisément dans le processus de modernisation et de transparence de la gestion du service public et dans la politique qui fait de l'évaluation un point clé essentiel. Elles apportent l'éclairage nécessaire permettant de faire émerger le débat démocratique objectif et de structurer les actions publiques.

Cette deuxième édition des indicateurs de l'enseignement est une nouvelle fois le fruit d'une étroite et riche collaboration entre la Commission de pilotage des enseignements organisés ou subventionnés par la Communauté française, le Service général du pilotage du système éducatif du Ministère de la Communauté française, l'Entreprise des Technologies Nouvelles de l'Information et de la Communication (ETNIC) et plusieurs experts et chercheurs universitaires.

Qu'ils reçoivent ici l'expression de toute ma gratitude pour le travail accompli.

Bonne lecture.

\section{Marie ARENA}

Ministre-Présidente de la Communauté française en charge de l'Enseignement obligatoire 



\section{GÉNÉRALITÉS}

p. 61 Participation des jeunes à l'enseignement de plein exercice et taux de scolarisation

p. 82 Origine géographique des effectifs scolaires de l'enseignement de plein exercice et en alternance

p. 103 Le coût de la scolarité à charge de la Communauté française

p. 124 Perspectives d'évolution de la population scolaire de l'enseignement fondamental et secondaire

\section{PUBLICS}

p. 145 Répartition différenciée des effectifs entre les différents niveaux, types et formes d'enseignement ordinaire

p. 166 Population scolaire, par réseau, dans l'enseignement fondamental et secondaire

p. 187 Disparités socioéconomiques dans l'enseignement fondamental et secondaire

p. 208 Implantations et effectifs scolaires en discrimination positive

p. 229 Publics de l'enseignement spécialisé : importance relative selon l'âge et le niveau socioéconomique

p. 2410 Publics de l'enseignement spécialisé : importance relative et répartition par type d'enseignement

p. 2611 Publics de l'enseignement spécialisé : les élèves de nationalité étrangère

p. 2812 Publics des différentes filières de l'enseignement secondaire ordinaire de plein exercice

\section{PARCOURS}

p. 3013 Retard scolaire dans l'enseignement ordinaire de plein exercice

p. 3214 Redoublement dans l'enseignement ordinaire de plein exercice

p. 3415 Changement d'établissement dans l'enseignement ordinaire de plein exercice

p. 3616 Attestations d'orientation délivrées par les Conseils de classe dans l'enseignement secondaire ordinaire de plein exercice

p. 3817 Parcours sur trois ans des élèves de première année de l'enseignement secondaire ordinaire

p. 4018 Flux d'élèves dans l'enseignement spécialisé

p. 4219 Taux d'accès à l'enseignement supérieur

\section{RÉSULTATS}

p. 4420 Compétences en lecture des élèves de $2^{\mathrm{e}}$ primaire -2007

p. 4621 Compétences en lecture des élèves de $5^{e}$ primaire -2007

p. 4822 Compétences en lecture des élèves de $2^{\mathrm{e}}$ secondaire -2007

p. 5023 Compétences en sciences des élèves de 15 ans - 2006

p. 5224 Obtention du Certificat d'Études de Base

p. 5425 Rythmes scolaires individuels et types de certification en $6^{\mathrm{e}}$ année de l'enseignement secondaire ordinaire de plein exercice

p. 5626 Taux de certification en $6^{\mathrm{e}}$ année de l'enseignement secondaire de plein exercice

p. 5827 Taux de réussite, de redoublement et d'abandon des étudiants de $1^{\text {re }}$ génération dans l'enseignement supérieur hors universités

p. 6028 Taux de réussite, de redoublement et d'abandon des étudiants de $1^{\text {re }}$ génération dans l'enseignement supérieur universitaire

\section{PERSONNELS}

p. 6229 Formation initiale des enseignants de l'enseignement obligatoire

p. 6430 Nombre de charges enseignantes de l'enseignement fondamental et secondaire ordinaires

p. 6631 Âge des enseignants de l'enseignement fondamental et secondaire

p. 6832 Féminisation du personnel enseignant de l'enseignement obligatoire 
Un peu plus de deux tiers des jeunes de 2 à 29 ans participent à l'enseignement. Ce résultat est principalement la conséquence de la scolarisation de la presque totalité des jeunes durant 15 ans, de 3 à 17 ans révolus.

En outre, en Communauté française, du niveau primaire au niveau supérieur, les écoles attirent des élèves provenant non seulement des pays étrangers, mais aussi des Communautés flamande et germanophone.

La mesure des taux de scolarisation par niveau d'enseignement met en évidence l'importance de l'accumulation progressive des retards dans l'enseignement primaire et surtout secondaire. Ainsi, $39 \%$ des personnes de 19 ans encore scolarisées en Communauté française le sont dans l'enseignement secondaire.

e $1^{\text {er }}$ janvier 2006, la Communauté française comptait un peu moins d'un million et demi de jeunes âgés de 2 à 29 ans ${ }^{1}$. Parmi ceux-ci, plus d'un million étaient scolarisés à temps plein. Par rapport à 1991-1992, on relève une croissance de plus de $7 \%$ de cette population scolaire à plein temps et une faible réduction de la population totale résidente de 2 à 29 ans. De ce fait, le taux brut de scolarisation ${ }^{2}$ à temps plein des jeunes de 2 à 29 ans est passé de $62,67 \%$ à $68,72 \%$ en quatorze ans.

Le calcul des taux de scolarisation par âge et par niveau d'enseignement permet de comparer la participation des jeunes de chaque classe d'âge aux différents niveaux d'enseignement et de mettre en évidence l'importance relative des groupes d'élèves et d'étudiants qui, par rapport à un cursus normal, sont en avance d'un an, "à l'heure » ou en retard d'une ou plusieurs années.

De 5 à 15 ans, les taux bruts sont systématiquement supérieurs à $100 \%$ au cours de la période observée (figure 2 ). Cette situation met en évidence des migrations d'élèves entre la Communauté française et les autres Communautés et pays limitrophes dont le solde est positif. A partir de 16 ans, on constate une diminution des taux bruts par rapport aux âges inférieurs mais les taux récents (2004-2005 et 2005-2006) sont supérieurs à ceux observés en 1991-1992, indiquant ainsi vraisemblablement pour ces âges une augmentation du taux net de scolarisation en Communauté française.

La figure 3 montre que la quasi-totalité des enfants sont inscrits à l'école maternelle à partir de 3 ans. À 5 ans, $2 \%$ ont déjà commencé des études primaires. À 11 ans, une même proportion d'enfants précoces sont inscrits en première année de l'enseignement secondaire.

En revanche, à 12 ans, un enfant sur quatre est toujours en primaire. Un an plus tard, à 13 ans, cette proportion est encore de $4 \%$. À 17 ans révolus, âge normal de fin d'enseignement secondaire et d'obligation scolaire, les étudiants en avance ne représentent plus qu'un pour cent et demi du total de la classe d'âge. Un an plus tard, à 18 ans, âge d'entrée dans l'enseignement supérieur, le taux de scolarisation à temps plein est de $88 \%$. Ce taux très élevé n'est cependant que la traduction d'un retard scolaire important puisque plus de la moitié des jeunes sont encore dans l'enseignement secondaire à 18 ans. Le taux d'élèves retardés dans le secondaire se résorbe ensuite lentement mais ne devient négligeable qu'à partir de 25 ans. Notons à ce sujet que $14 \%$ environ des jeunes de 20 ans suivant un enseignement de plein exercice sont encore dans le secondaire.

Avec des taux de scolarisation compris entre $32 \%$ et $50 \%$ pour les jeunes de 18 à 22 ans recensés dans l'enseignement supérieur, la Communauté française occupe une des premières places parmi les pays de I'OCDÉ. Cette position est due en grande partie au développement de l'enseignement de type court permettant à de nombreux élèves de poursuivre des études supérieures, type d'études qui, dans cette tranche d'âge, absorbe la moitié des étudiants de l'enseignement supérieur.

La comparaison avec l'année 1991-1992 montre aussi une croissance de plus de $20 \%$ des taux de participation à l'enseignement des jeunes de 21 ans et plus. Cette croissance, à partir de 23 ans, est supérieure dans l'enseignement supérieur de type court à celle observée dans I'enseignement de niveau universitaire.

1 Voir le tableau de la figure 1.

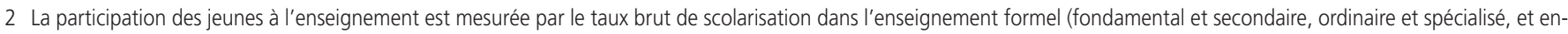
seignement supérieur de plein exercice). Afin de répondre aux définitions internationales (EUROSTAT, OCDÉ), il est également tenu compte de l'enseignement en alternance dans le calcul des taux. Par contre, par manque de données statistiques précises, les élèves en apprentissage (Classes Moyennes par exemple) n'ont pu être recensés. Le taux brut ainsi calculé est le résultat, exprimé en pourcentage, du rapport entre la population scolarisée dans une entité territoriale et la population de même âge résidant dans la même entité. Le volume de la population scolaire dépend du nombre de jeunes scolarisables dans l'entité, de leur taux net de scolarisation et, dans une moindre mesure, du solde des migrations d'élèves. Le taux brut de scolarisation intègre ces deux derniers facteurs explicatifs.

L'âge considéré est exprimé en années révolues au 1er janvier. La population totale est celle inscrite au Registre national le 1 er janvier. En Région bruxelloise, la population a été répartie linguistiquement selon la clé : 85 \% pour la Communauté française / 15 \% pour la Communauté flamande. 
1.1 Participation à l'enseignement de 1991-1992 à 2005-2006

\begin{tabular}{|c|c|c|c|c|c|c|c|}
\hline \multirow[t]{2}{*}{ Communauté française } & \multirow{2}{*}{$\begin{array}{c}\text { Total } \\
\text { Effectif }\end{array}$} & \multicolumn{2}{|c|}{$\begin{array}{l}\text { opulation résidente } \\
\qquad 2 \text { à } 29 \text { ans }\end{array}$} & \multirow{2}{*}{$\begin{array}{l}\text { Total } \\
\text { Effectif }\end{array}$} & \multicolumn{2}{|c|}{$\begin{array}{c}\text { Population scolaire } \\
2 \text { à } 29 \text { ans }\end{array}$} & \multirow{2}{*}{$\begin{array}{l}\text { Taux brut de } \\
\text { participation } \\
2 \text { à } 29 \text { ans }\end{array}$} \\
\hline & & Effectif & $\%$ & & Effectif & $\%$ & \\
\hline Année 1991-1992 & 4016273 & 1495923 & 37,25 & 942900 & 937465 & 99,42 & 62,67 \\
\hline Indice & 100 & 100 & & 100 & 100 & & \\
\hline Année 1995-1996 & 4051034 & 1470115 & 36,29 & 977519 & 968676 & 99,1 & 65,89 \\
\hline Indice & 100,87 & 98,27 & & 103,67 & 103,33 & & \\
\hline Année 1999-2000 & 4084105 & 1448273 & 35,46 & 977317 & 969143 & 99,16 & 66,92 \\
\hline Indice & 101,69 & 96,82 & & 103,65 & 103,38 & & \\
\hline Année 2004-2005 & 4179167 & 1450901 & 34,72 & 1011582 & 1000407 & 98,9 & 68,95 \\
\hline Indice & 104,06 & 96,99 & & 107,28 & 106,71 & & \\
\hline Année 2005-2006 & 4206842 & 1460175 & 34,71 & 1014314 & 1003492 & 98,9 & 68,72 \\
\hline Indice & 104,74 & 97,61 & & 107,57 & 107,04 & & \\
\hline
\end{tabular}

En 2005-2006, 1003492 jeunes de 2 à 29 ans ont participé à l'enseignement en Communauté française. Ils représentent 68,72 \% de la population résidente de la même tranche d'âge.

1.2 Taux brut de scolarisation dans l'enseignement en Communauté française - Comparaisons des années scolaires 1991-1992, 2004-2005 et 2005-2006

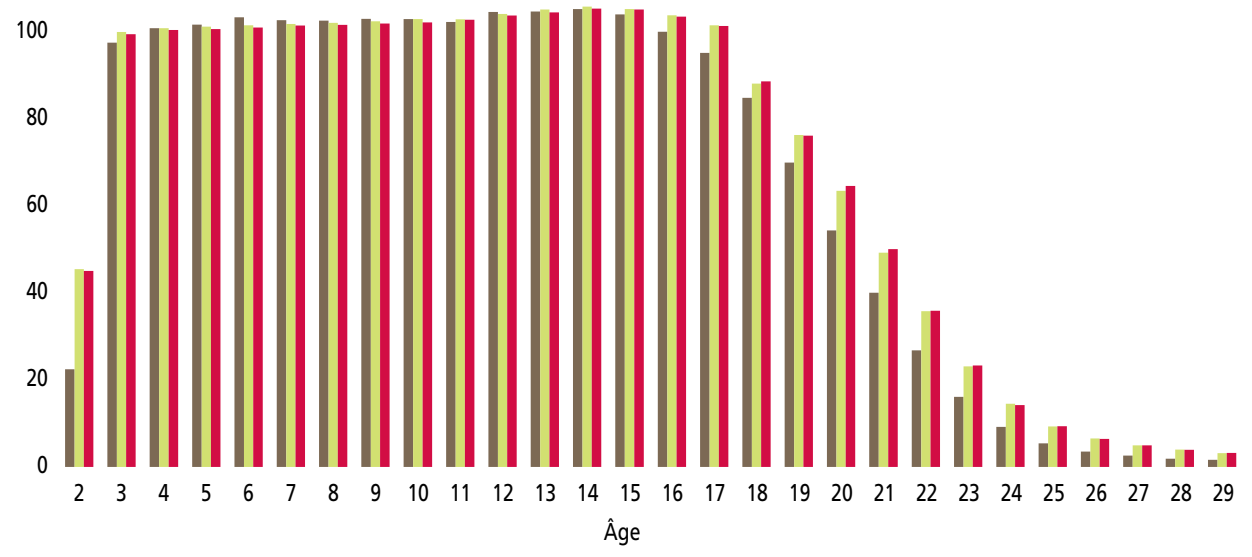

1991-1992

2004-2005

2005-2006

À l'âge de 21 ans, le taux brut de scolarisation dans l'enseignement en Communauté française était de $40 \%$ en 1991-1992, il est actuellement de $50 \%$; à l'âge de 2 ans, le passage du taux brut de $22 \%$ en 1991-1992 à $45 \%$ en 20052006 s'explique par un comptage des enfants scolarisés à des dates différentes (octobre dans le premier cas et janvier pour les deux dernières années).

\subsection{Taux brut de scolarisation par niveau dans l'enseignement en Communauté française en 2005-2006}

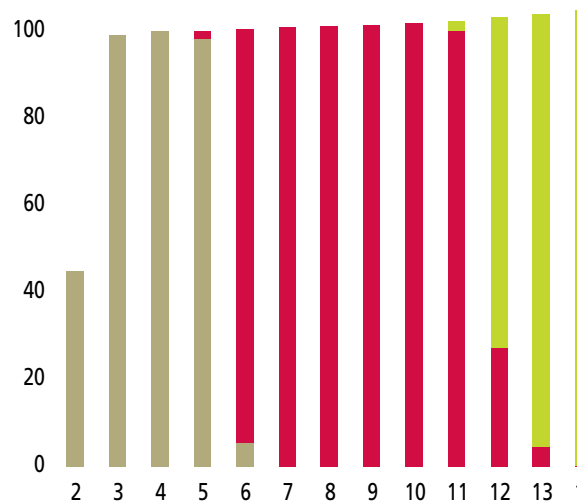

100

2

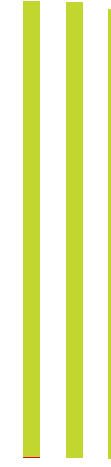

$14 \quad 15$
Préscolaire

Primaire

Secondaire

Supérieur de type court

Supérieur de niveau universitaire

À 12 ans, le taux brut de scolarisation dans l'enseignement en Communauté française est de $103,3 \%$, dont $76 \%$ correspond à une inscription dans l'enseignement secondaire et 27,3\% à une inscription dans l'enseignement primaire. Le taux brut supérieur à $100 \%$ indique qu'à cet âge le nombre d'élèves inscrits dans les écoles de la Communauté française est supérieur à la population résidente. 


\section{Origine géographique des effectifs scolaires de l'enseignement de plein exercice et en alternance}

Dans l'enseignement ordinaire obligatoire, les élèves domiciliés à l'étranger représentent moins de $3 \%$ des inscriptions dans les écoles de la Communauté française tandis qu'ils en représentent $13 \%$ dans l'enseignement supérieur.

Un peu moins de $2 \%$ des enfants de 3 à 15 ans domiciliés en Région wallonne fréquentent les écoles de la Communauté flamande tandis que 2,3 \% des enfants de cette même tranche d'âge domiciliés en Région flamande fréquentent les écoles de la Communauté française.

Les enfants de 3 à 15 ans domiciliés en Région de Bruxelles-Capitale fréquentent pour leur part les écoles de la Communauté française à concurrence de $81 \%$.

e financement de la Communauté française est en partie établi en fonction du nombre d'élèves de 6 à 17 ans régulièrement inscrits dans ses établissements scolaires. La construction de scénarios portant sur des perspectives d'évolution des populations scolaires ${ }^{1}$ se révèle donc indispensable à l'établissement des perspectives budgétaires. Parmi les facteurs susceptibles d'influencer le nombre d'élèves inscrits dans les écoles de la Communauté française figure en bonne place l'origine géographique des élèves.

A cet égard, la figure 1 présente, par forme et niveau d'enseignement, la répartition des élèves selon qu'ils sont domiciliés en Région wallonne, en Région bruxelloise, en Région flamande ou à l'étranger. L'intérêt essentiel de cette figure réside dans l'observation de la part des élèves domiciliés à l'étranger et fréquentant notre système d'enseignement. C'est cette part qui, dans la figure 1, varie le plus selon le niveau et la forme d'enseignement : elle ne représente que $1 \%$ de la population scolarisée dans l'enseignement fondamental ordinaire tandis qu'elle en représente $3 \%$ dans le secondaire ordinaire, $8 \%$ dans le spécialisé et $13 \%$ dans le supérieur.

En ce qui concerne les élèves domiciliés en Belgique, les scénarios d'évolution de la population scolarisée dans l'enseignement de la Communauté française doivent plutôt se baser sur les taux de participation présentés à la figure 2 . Ces taux de participation déterminent la proportion des jeunes d'un âge donné et domiciliés dans une région belge donnée qui se scolarisent dans les écoles de la Communauté française.

Il apparaît ainsi qu'en Région wallonne hors Communauté germanophone, le taux de participation des élèves de 3 à 15 ans dans l'enseignement de la Communauté française est de $98 \%$. Les $2 \%$ restants correspondent à une part d'enfants scolarisés dans des écoles de la Communauté flamande ou germanophone ou dans des écoles privées ou, encore, non scolarisés. Au delà de l'âge de scolarisation obligatoire, le taux de participation baisse de plus en plus suite aux sorties du système scolaire de plein exercice. A l'âge de 18 ans, $79 \%$ des jeunes sont encore inscrits dans une école de plein exercice de la Communauté française (50\% dans le secondaire et $29 \%$ dans le supérieur). A 20 ans, ils ne sont plus que $53 \%$, dont $11 \%$ toujours inscrits dans le secondaire et $42 \%$ inscrits dans le supérieur. C'est à 20 ans que le taux de participation à l'enseignement supérieur est le plus élevé.

En Région de Bruxelles-Capitale, le taux de participation des élèves de 3 à 15 ans dans l'enseignement de la Communauté française se situe entre $78 \%$ (à 3 ans) et $84 \%$ (à 15 ans). En dehors d'une fraction marginale d'enfants inscrits dans des écoles privées ou non scolarisés, le reste, soit entre 16 et $22 \%$, est scolarisé dans des écoles de la Communauté flamande. Comme en Région wallonne, au delà de l'âge de scolarisation obligatoire, le taux de participation baisse de plus en plus suite aux sorties du système scolaire de plein exercice. A 18 ans, $68 \%$ des jeunes domiciliés en Région bruxelloise sont inscrits dans un établissement de la Communauté française, dont $49 \%$ dans le secondaire et $19 \%$ dans le supérieur. Dans la tranche d'âge des 18 à 21 ans, le rapport secondaire/supérieur est plus élevé que celui observé en Région wallonne. Une autre différence concerne le choix des études supérieures : la population domiciliée en Région de Bruxelles-Capitale choisit proportionnellement davantage l'université que le supérieur hors universités. A l'âge de 18 ans, $46 \%$ des étudiants de l'enseignement supérieur domiciliés en Région wallonne sont inscrits à l'université et $54 \%$ dans le supérieur hors universités alors que $60 \%$ des étudiants de l'enseignement supérieur domiciliés en Région bruxelloise sont inscrits à l'université et $40 \%$ dans le supérieur hors universités ; cet écart entre les deux régions se maintient jusqu'à 21 ans inclus.

Dans les deux autres communautés, le taux de participation à l'enseignement de la Communauté française est évidemment nettement plus faible. En Région flamande, le taux de participation maximum est de $3 \%$, dans la tranche d'âge des 13 à 18 ans. En Communauté germanophone, le taux de participation est de l'ordre de 2 à $4 \%$ dans la tranche d'âge des 2 à 11 ans (enseignement fondamental) et de l'ordre de $10 \%$ dans la tranche d'âge des 12 à 17 ans (enseignement secondaire) ; le taux de participation de la population germanophone à l'enseignement supérieur de la Communauté française s'élève par contre à plus de $20 \%$ dans la tranche d'âge des 18 à 20 ans.

1 Voir Indicateur 4 - Perspectives d'évolution de la population scolaire de l'enseignement fondamental et secondaire. 
Fondamental ordinaire

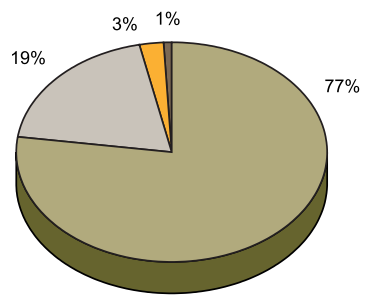

Spécialisé

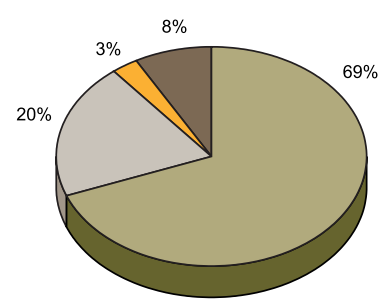

Secondaire ordinaire

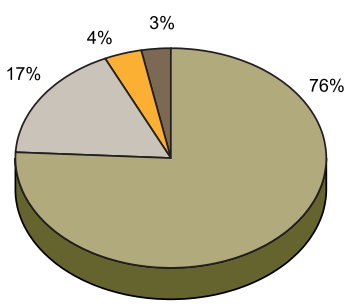

Supérieur

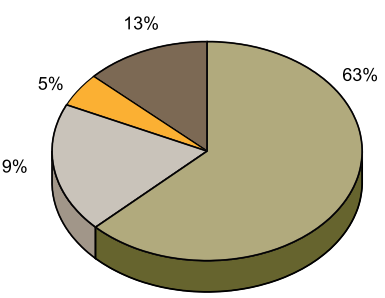

Région wallonne

Région Bruxelles-Capitale

Région flamande

À l'étranger

Dans l'enseignement spécialisé, $69 \%$ des élèves sont domiciliés en Région wallonne, $20 \%$ en Région bruxelloise, $3 \%$ en Région flamande et $8 \%$ sont domiciliés à l'étranger.

2.2 Taux de participation à l'enseignement de plein exercice et en alternance de la Communauté française des élèves domiciliés en Belgique, par région de domicile - Année scolaire 2005-2006

Région wallonne hors communes germanophones

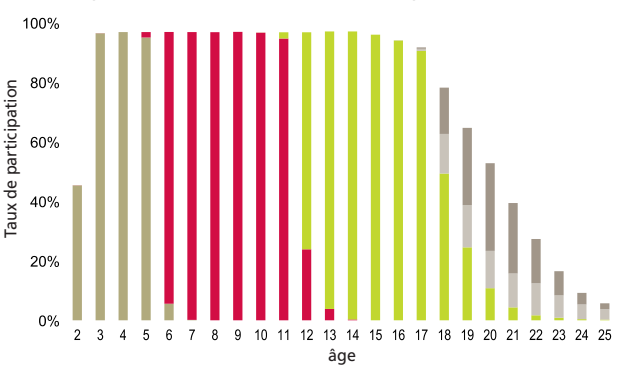

$100 \%$

Région de Bruxelles-Capitale

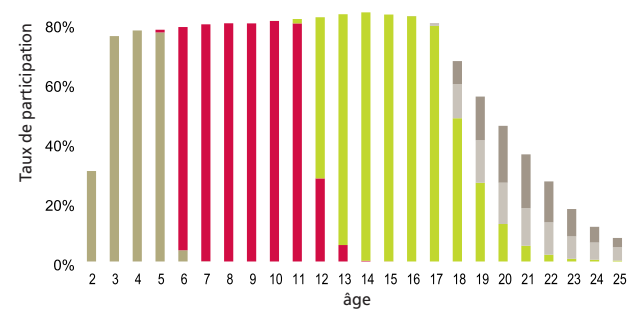

Communes germanophones

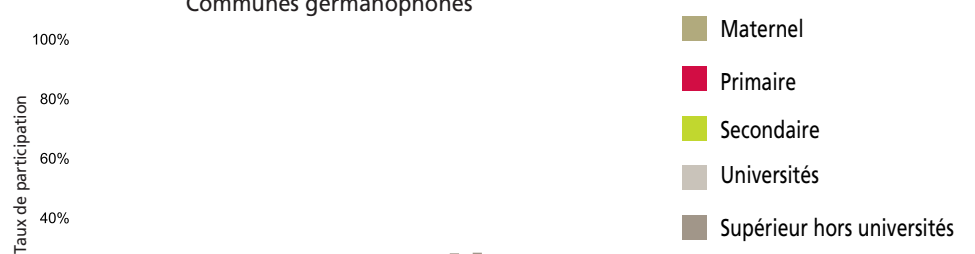

$20 \%$

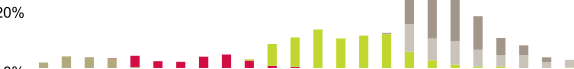

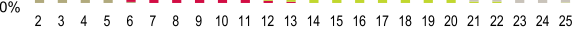
âge

Région flamande

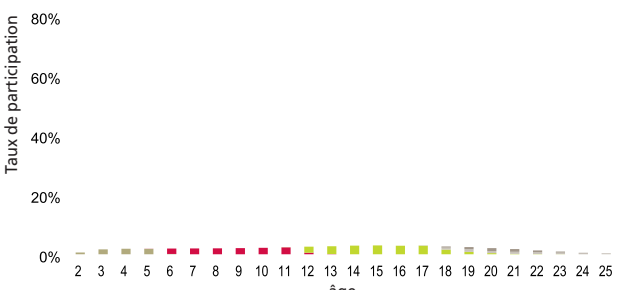

En Région wallonne hors communes germanophones, $97 \%$ de la population âgée de 15 ans est présente dans l'enseignement obligatoire de la Communauté française; ce est de $84 \%$ en Région bruxelloise, $10 \%$ dans les communes germanophones et $3 \%$ en Région flamande. même taux de participation 


\section{Le coût de la scolarité à charge de la Communauté française}

En 2006, la Communauté française de Belgique a consacré 5,7 milliards d'euros à son système éducatif. La dépense moyenne par étudiant varie de 2500 euros à 6000 euros dans l'enseignement obligatoire ordinaire et de 4900 euros à 8000 euros dans l'enseignement supérieur. L'enseignement spécialisé coûte quant à lui 12200 euros par élève, en moyenne.

Ainsi, une scolarité menée sans redoublement de la maternelle au terme de l'enseignement secondaire supérieur s'évalue à 64300 euros par élève (à charge de la Communauté française). Le coût moyen d'un baccalauréat peut varier de 79000 à 88700 euros tandis que celui d'un master (en 5 ans) peut varier de 88300 à 104400 euros selon qu'il est effectué hors ou au sein d'une université, sans retard scolaire depuis la maternelle.

En 2005-2006, le retard scolaire a généré un surcoût d'environ 335 millions d'euros dans l'enseignement obligatoire ordinaire.

e budget annuel global de l'enseignement à charge de la Communauté française s'élève, en 2006, à 5689 millions d'euros.

Le coût annuel par élève ou étudiant en 2006 est obtenu en divisant, par les effectifs 2005-2006, la partie du budget global destinée à l'enseignement lui-même (hors services communs, affaires générales, recherche en éducation, pilotage de l'enseignement, bâtiments scolaires, recherche scientifique, allocations et prêts d'études, inspection médicale...). Le coût annuel moyen par élève a été calculé globalement par niveau de scolarité indépendamment de la forme d'enseignement (générale, technique ou professionnelle) suivie dans le secondaire et indépendamment du type (court ou long) et du domaine d'études suivies dans le supérieur. Par conséquent, il convient de nuancer les coûts globaux ainsi présentés dans la figure 2 par le fait que la Communauté française octroiera à l'école des dotations ou subventions de fonctionnement plus importantes pour un élève du secondaire qualifiant (technique ou professionnel), au vu des équipements nécessaires à son apprentissage. De la même façon, les dotations ou subventions de fonctionnement attribuées aux établissements d'enseignement supérieurs sont pondérées en fonction du type d'enseignement (court ou long) et du domaine d'études suivies, générant des dépenses annuelles plus importantes pour certains domaines d'études.

A prix constants (figure 1), le coût moyen par élève dans l'enseignement fondamental ordinaire a augmenté d'environ $2 \%$ par an sur la période observée (1997-1998 à 2005-2006). Dans l'enseignement spécialisé, une augmentation s'est également produite au cours des trois dernières années, le coût moyen par élève passant de l'indice 100,8 en 2002-2003 à l'indice 108,5 en 2005-2006 (base 100 en 1997-1998). Dans l'enseignement supérieur, on observe par contre une baisse du coût moyen par étudiant (indice 98,2 dans l'enseignement supérieur hors universités et indice 96,4 dans l'enseignement universitaire en 2005-2006). A prix courants (figure 2), le coût moyen de l'année scolaire à charge de la Communauté française s'étend de 2548 euros par élève de l'enseignement maternel à 8008 euros par étudiant de l'enseignement universitaire, en 2005-2006. Le coût annuel moyen le plus important, 12185 euros par élève, est engendré par l'enseignement spécialisé.

Le cumul des dépenses annuelles par niveau d'enseignement dans la figure 3 permet de calculer la dépense théorique totale de plusieurs scolarités types, sans redoublement. Ainsi, par exemple, pour un élève quittant l'enseignement secondaire après 15 ans de scolarité (du maternel à la fin du secondaire), 64315 euros seront dépensés en moyenne par la Communauté française. Le coût de l'enseignement spécialisé sur une durée de 15 ans représenterait, quant à lui, un montant moyen de 182781 euros. En ce qui concerne l'enseignement supérieur, les cursus afférents au baccalauréat et au master sont organisés pour la première fois en 2007-2008, ils peuvent aussi bien être suivis dans un établissement d'enseignement supérieur que dans une université. Ces deux enseignements génèrent des coûts par étudiant différents. Dès lors, les études menant au titre de bachelier (en 3 ans) et de master (en 5 ans) engendrent un coût moyen total de 78920 et de 88657 euros si elles sont menées dans un établissement supérieur hors universités. Les mêmes titres obtenus à l'université s'évaluent respectivement en moyenne à 88339 et 104355 euros. Il convient d'interpréter ces divergences de coût en gardant à l'esprit le principe de financement de l'enseignement supérieur par enveloppe fermée avec, pour conséquence, un coût moyen par étudiant principalement influencé par le nombre d'étudiants. Puisque les étudiants sont plus nombreux en établissements supérieurs hors universités (les trois quarts suivent du type court), le coût moyen de cet enseignement par étudiant est plus faible, à enveloppe constante.

Au-delà du coût théorique, il y a lieu de prendre en compte les coûts supplémentaires engendrés par le redoublement et le retard scolaire qui en découle. Dans l'enseignement obligatoire ordinaire, ces coûts peuvent être approchés en imputant le coût moyen d'une année supplémentaire à chaque redoublant. Comme le montre la figure 4 , les retards scolaires auraient généré un surcoût s'élevant à environ 44,8 millions d'euros dans le primaire et à 289,9 millions d'euros dans le secondaire en 2005-2006, soit 5,9 \% au total du budget global de l'enseignement. Ces surcoûts sont probablement sous-estimés au niveau du secondaire par le fait que les taux de redoublement les plus élevés s'observent dans l'enseignement qualifiant (en $3 e, 4 e$ et 5 e années), plus cher. Le fait que les dépenses liées au redoublement n'ont cessé de croître depuis 10 ans est en partie expliqué par l'évolution du taux de redoublement lui-même qui est passé, entre 1997-1998 et 2005-2006, de 3,7 \% à 4,4 \% dans le primaire et de $10,4 \%$ à $13,4 \%$ dans le secondaire. 
3.1 Evolution du coût annuel moyen par élève/étudiant à charge de la Communauté française, à prix constants (base 100 de 1997)

\begin{tabular}{|c|c|c|c|c|c|c|c|c|c|}
\hline Coût moyen à prix constants (base 100) & 1997-1998 & 1998-1999 & $1999-2000$ & $2000-2001$ & 2001-2002 & 2002-2003 & 2003-2004 & $2004-2005$ & $2005-2006$ \\
\hline FONDAMENTAL & 100,0 & 103,1 & 104,9 & 106,9 & 108,8 & 111,0 & 113,8 & 117,7 & 121,0 \\
\hline Maternel & 100,0 & 105,7 & 109,1 & 113,4 & 117,3 & 121,8 & 118,6 & 115,5 & 118,5 \\
\hline Primaire & 100,0 & 103,3 & 107,2 & 112,6 & 116,9 & 121,1 & 112,3 & 113,6 & 116,2 \\
\hline SECONDAIRE & 100,0 & 101,7 & 100,9 & 100,2 & 98,6 & 98,3 & 98,3 & 101,0 & 101,5 \\
\hline SPECIALISE & 100,0 & 101,5 & 100,9 & 102,0 & 100,7 & 100,8 & 105,1 & 107,7 & 108,5 \\
\hline SUPÉRIEUR (hors universités) & 100,0 & 100,2 & 98,7 & 101,2 & 101,5 & 101,0 & 100,1 & 97,3 & 98,2 \\
\hline UNIVERSITES & 100,0 & 100,9 & 101,2 & 101,7 & 100,4 & 98,3 & 97,2 & 96,0 & 96,4 \\
\hline
\end{tabular}

Le coût annuel moyen à charge de la Communauté française d'un élève de l'enseignement fondamental a augmenté de 21 \% de $1997-1998$ à $2005-2006$.

\section{2 Évolution du coût annuel moyen par élève/étudiant à charge de la Communauté française, à prix courants, de 1997-1998 à 2005-2006}

\begin{tabular}{|c|c|c|c|c|c|c|c|c|c|}
\hline Coût moyen à euros courants & $1997-1998$ & $1998-1999$ & $1999-2000$ & $2000-2001$ & $2001-2002$ & $2002-2003$ & $2003-2004$ & $2004-2005$ & $2005-2006$ \\
\hline FONDAMENTAL & 2252 & 2345 & 2430 & 2543 & 2636 & 2727 & 2842 & 2947 & 3078 \\
\hline Maternel & 1903 & 2012 & 2076 & 2158 & 2233 & 2318 & 2502 & 2448 & 2548 \\
\hline Primaire & 2428 & 2507 & 2603 & 2733 & 2839 & 2939 & 3023 & 3238 & 3387 \\
\hline SECONDAIRE & 5177 & 5314 & 5374 & 5483 & 5491 & 5554 & 5645 & 5925 & 6058 \\
\hline SPECIALISE & 9745 & 9985 & 10109 & 10502 & 10556 & 10713 & 11352 & 11887 & 12185 \\
\hline SUPÉRIEUR (hors universités) & 4300 & 4347 & 4365 & 4596 & 4694 & 4737 & 4772 & 4741 & 4868 \\
\hline UNIVERSITES & 7204 & 7340 & 7500 & 7739 & 7781 & 7726 & 7761 & 7835 & 8008 \\
\hline
\end{tabular}

En 2005-2006, le coût annuel moyen à charge de la Communauté française d'un élève de l'enseignement spécialisé s'élève à 12185 euros.

3.3 Coûts annuels de scolarités types à charge du budget de la Communauté française (2005-2006)

\begin{tabular}{|c|c|c|c|c|c|c|c|c|c|}
\hline & \multicolumn{4}{|c|}{ Enseignement obligatoire } & \multicolumn{4}{|c|}{ Enseignement supérieur } & \\
\hline & \multirow{2}{*}{$\begin{array}{l}\text { Maternel } \\
\text { ordinaire }\end{array}$} & \multirow{2}{*}{$\begin{array}{l}\text { Primaire } \\
\text { ordinaire }\end{array}$} & \multirow{2}{*}{$\begin{array}{c}\text { Secondaire } \\
\text { ordinaire }\end{array}$} & \multirow[t]{2}{*}{ Spécialisé } & \multicolumn{2}{|c|}{ Baccalauréat } & \multicolumn{2}{|c|}{ Master } & \\
\hline & & & & & Hors Univ. & Univ. & Hors Univ. & Univ. & \\
\hline Coût annuel/ élève-étudiant (en euros) & 2548 & 3387 & 6058 & 12185 & 4868 & 8008 & 4868 & 8008 & \\
\hline \multicolumn{9}{|l|}{ SCOLARITES TYPES } & $\begin{array}{l}\text { Coût TOTAL } \\
\text { (en euros) }\end{array}$ \\
\hline Secondaire ordinaire & 3 ans & 6 ans & 6 ans & & & & & & 64315 \\
\hline Secondaire spécialisé & & & & 15 ans & & & & & 182781 \\
\hline Baccalauréat hors universités & 3 ans & 6 ans & 6 ans & & 3 ans & & & & 78920 \\
\hline Master hors universités & 3 ans & 6 ans & 6 ans & & 3 ans & & 2 ans & & 88657 \\
\hline Baccalauréat universités & 3 ans & 6 ans & 6 ans & & & 3 ans & & & 88339 \\
\hline Master universités & 3 ans & 6 ans & 6 ans & & & 3 ans & & 2 ans & 104355 \\
\hline
\end{tabular}

En 2005-2006, le coût théorique (sans redoublement) à charge de la Communauté française d'un master obtenu dans une université s'élève à 104355 euros en moyenne par étudiant.

3.4 Évolution du taux du redoublement de l'enseignement primaire et secondaire ordinaires et du coût annuel engendré par le retard scolaire à charge du budget de la Communauté française de 1997-1998 à 2005-2006

\begin{tabular}{|c|c|c|c|c|c|c|c|c|c|}
\hline Taux de redoublement & $1997-1998$ & 1998-1999 & $1999-2000$ & $2000-2001$ & $2001-2002$ & $2002-2003$ & $2003-2004$ & $2004-2005$ & $2005-2006$ \\
\hline Primaire Ordinaire & $3,7 \%$ & $3,4 \%$ & $3,5 \%$ & $3,3 \%$ & $3,4 \%$ & $4,0 \%$ & $4,1 \%$ & $4,5 \%$ & $4,4 \%$ \\
\hline Secondaire Ordinaire & $10,4 \%$ & $9,8 \%$ & $10,4 \%$ & $10,5 \%$ & $11,4 \%$ & $12,8 \%$ & $12,7 \%$ & $13,2 \%$ & $13,4 \%$ \\
\hline Coût redoublement (euros courants): & 1997-1998 & 1998-1999 & $1999-2000$ & $2000-2001$ & $2001-2002$ & $2002-2003$ & 2003-2004 & $2004-2005$ & $2005-2006$ \\
\hline Primaire Ordinaire & 28635972 & 27159705 & 29178851 & 28808553 & 30325697 & 36534223 & 37572298 & 44360600 & 44767262 \\
\hline Secondaire Ordinaire & 182376355 & 176517583 & 187086819 & 194678749 & 213167731 & 246455121 & 254115151 & 279362920 & 289901867 \\
\hline
\end{tabular}

En 2005-2006, le coût du retard scolaire à charge de la Communauté française s'élève à 44762262 euros dans l'enseignement primaire et à 289901867 euros dans l'enseignement secondaire. 


\section{Perspectives d'évolution de la population scolaire de l'enseignement fondamental et secondaire}

Le scénario retenu en matière de perspectives de population scolaire fait apparaître, à l'horizon 2024-2025, une diminution de plus de 6 \% des effectifs au niveau secondaire, dont $4 \%$ de perte sur les 8 prochaines années.

En revanche, la population de l'enseignement fondamental diminuerait de 2 \% à l'horizon 2015-2016 et augmenterait à due concurrence à l'horizon 2024-2025.

n appliquant les perspectives démographiques établies dans l'étude ${ }^{1}$ Co l'Institut National des Statistiques (INS), un scénario d'évolution de population a été appliqué aux élèves recensés en Communauté française. Les perspectives de population ont été étudiées pour l'enseignement fondamental et secondaire (en ce compris le spécialisé et I'alternance) de la manière suivante.

Le taux de scolarisation, par âge et par région de domiciliation, observé dans les écoles organisées ou subventionnées par la Communauté française en 2005-2006 a été appliqué sur les perspectives démographiques à l'horizon 2024-2025. Le scénario choisi maintient constants les flux entrants et sortants d'élèves domiciliés à l'étranger étudiant en Communauté française et la migration des élèves entre régions ${ }^{2}$.

La figure 1 décrit, pour l'année de départ 2005-2006, la répartition par région de la population scolaire entre les niveaux maternel, primaire et secondaire, ordinaire et spécialisé, en Communauté française. On y recense une répartition assez semblable dans les trois niveaux d'enseignement qui, au total, comprennent $5 \%$ d'élèves domiciliés en Région flamande ou hors Belgique.

La figure 2 présente la perspective sur 20 ans calculée à partir d'une base de 100 élèves dans chaque niveau, en 2004-2005. Elle révèle une diminution de la population scolaire (toutes régions confondues) qui atteindrait, au total, un maximum de 3,2 \% en 2018-2019. Cette diminution serait essentiellement due à la baisse des effectifs aux niveaux primaire et secondaire. Sur les 6 années suivantes, la population de l'enseignement maternel tendrait à dépasser son effectif de départ tandis que celle de l'enseignement primaire rejoindrait son niveau de départ. Au secondaire, par contre, une diminution systématique des effectifs devrait atteindre, en 20 ans, plus de $6 \%$ de la population actuelle.

Les figures 4.3.a, 4.3.b et 4.3.c décrivent l'évolution de la variation annuelle des populations aux trois niveaux d'enseignement. Dans le maternel, une diminution de $1,8 \%$ de l'effectif actuel est prévue dans les 8 prochaines années après quoi la tendance s'inverserait principalement durant les 7 années suivantes. Dans le primaire, les effectifs devraient être globalement en baisse sur les 13 ans à venir (avec une perte de 2,7 \% de l'effectif actuel). Une augmentation progressive est prévue dans les années suivantes. Dans le secondaire, les effectifs seraient systématiquement en baisse dans les 19 prochaines années. Des variations importantes sont prévues durant les 10 premières années étudiées avec une perte potentielle de 3,9\% de l'effectif actuel. Durant les dix années suivantes, une nouvelle diminution de $2,1 \%$ de l'effectif serait observée.

Ces perspectives à long terme nécessiteront d'être adaptées périodiquement aux dernières hypothèses démographiques de I'INS.

1 Démographie mathématique - Perspectives de population 2000-2050 - Direction générale Statistique et Information économique - Service Démographie - Octobre 2001 La base de départ de ces perspectives de la population est la situation au $1^{\text {er }}$ janvier 2000. Outre les quatre mouvements démographiques (mortalité, fécondité, migrations internes et externes), ces perspectives tiennent compte de la naturalisation et de la régularisation des sans papiers. Les calculs sont effectués par sexe, par âge et par arrondissement.

2 Une note méthodologique complète ainsi que des tableaux de résultats des « Perspectives de populations scolaires 2006-2007 à 2024-2025 » se trouvent sur le site http://www.statistiques.cfwb.be/rapports.php. 
4.1 Répartition des effectifs selon le domicile de l'élève par niveau d'enseignement - Année scolaire 2005-2006

\begin{tabular}{|c|c|c|c|c|}
\hline Région de domicile de l'élève & Niveau Maternel & Niveau Primaire & Niveau Secondaire & $\begin{array}{l}\text { Enseignement } \\
\text { fondamental et } \\
\text { secondaire }\end{array}$ \\
\hline Effectifs 2005-2006 & 176146 & 315133 & 372804 & 864083 \\
\hline Région de Bruxelles-Capitale & $20,0 \%$ & $19,1 \%$ & $17,3 \%$ & $18,5 \%$ \\
\hline Région wallonne & $77,0 \%$ & $77,1 \%$ & $75,6 \%$ & $76,4 \%$ \\
\hline Région flamande & $2,2 \%$ & $2,8 \%$ & $3,8 \%$ & $3,1 \%$ \\
\hline Hors - Belgique & $0,8 \%$ & $1,0 \%$ & $3,3 \%$ & $2,0 \%$ \\
\hline TOTAL & $100 \%$ & $100 \%$ & $100 \%$ & $100 \%$ \\
\hline
\end{tabular}

\subsection{Perspectives 2006-2007 à 2024-2025 d'évolution du nombre d'élèves (en base 100 en 2004-2005) dans les écoles fondamentales et secondaires de la Communauté française}

105

100

95

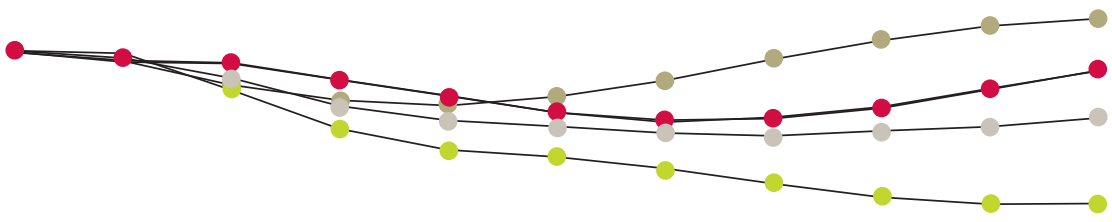

90

2004-2005 2006-2007 2008-2009 2010-2011 2012-2013 2014-2015 2016-2017 2018-2019 2020-2021 2022-2023 $2024-2025$

\section{- - Niveau maternel \\ - - Niveau primaire \\ - - Niveau secondaire \\ - Tous niveaux}

En Communauté française, la population scolaire dans l'enseignement fondamental et secondaire (tous niveaux) mise à l'indice 100 en 2004-2005 passe à un minimum de 96,8 en 2018-2019 (soit une prévision de perte de $3,2 \%$ de la population scolaire en 12 ans) et remonte à 97,5 en 2024-2025.

\subsection{Evolution de la variation annuelle de la population scolaire dans les écoles de la Communauté française - Perspectives 2006-2007 à 2024-2025}

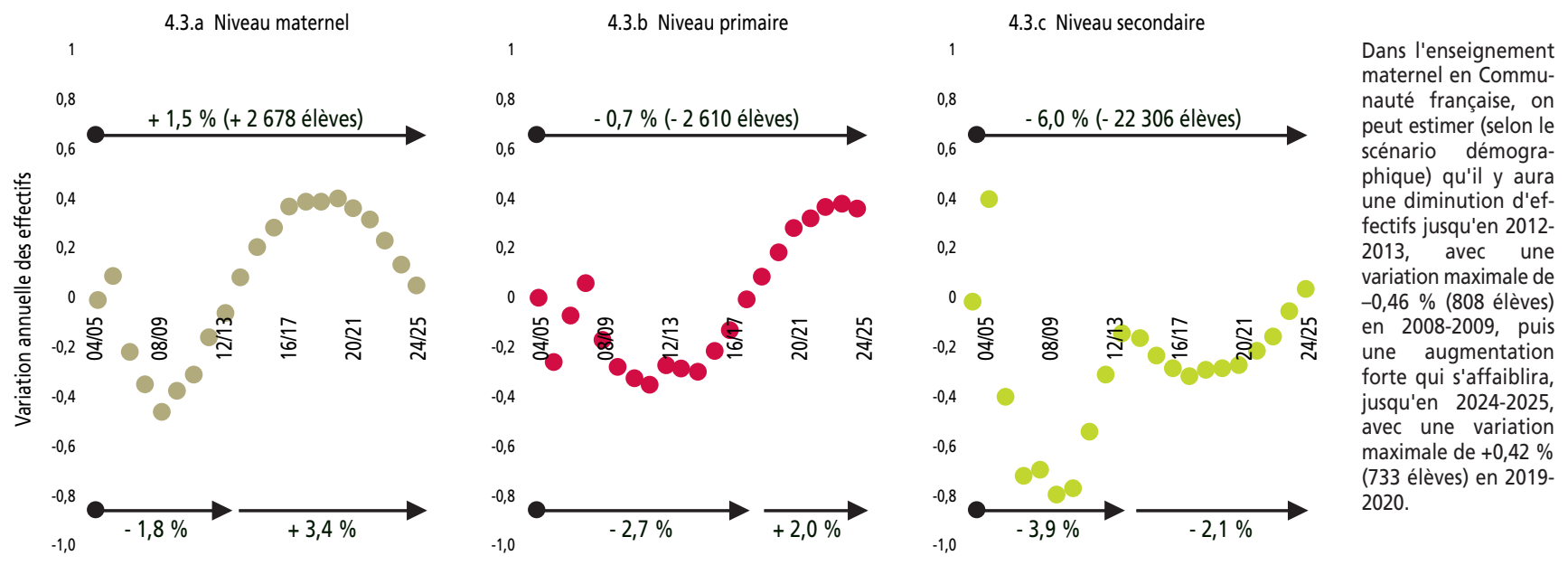


L'examen simultané de la différenciation des élèves selon le retard, le sexe et la nationalité fait apparaître des discriminations progressives durant le parcours scolaire, principalement à l'entame du secondaire et ensuite lors du choix des formes techniques et professionnelle, au début du deuxième degré. II s'agit là des deux principaux moments de l'orientation et du développement du phénomène de discrimination rencontrés lors du cheminement scolaire.

e Décret « Missions » de 1997 prévoit un accès égal à toutes les formations pour les filles et les garçons et une absence de hiérarchie entre établissements, formes et sections d'enseignement ${ }^{1}$. Dans cette perspective, un indicateur permettant de mesurer l'importance, I'homogénéité et le recours aux différentes composantes de l'offre scolaire consiste à mesurer par forme, section et année d'études quelques caractéristiques individuelles des élèves. Trois caractéristiques ont été retenues afin d'illustrer la répartition différenciée des effectifs au sein des diverses composantes du système d'enseignement : le retard scolaire, le sexe et la nationalité.

Pour chaque variable, un taux a été calculé pour chaque année d'études de l'enseignement fondamental et secondaire ordinaire et de I'enseignement en alternance (CEFA). Sur la base des taux obtenus pour chaque variable, les années d'études ont été systématiquement réparties en cinq catégories de taux permettant de distinguer par un graphique les classes extrêmes et la classe médiane de la distribution des taux relatifs à chacune des trois variables du système d'enseignement.

Le système d'enseignement ordinaire se caractérise d'abord par un taux de retard scolaire élevé ${ }^{2}$. Ainsi, en $1{ }^{\text {re }}$ primaire, $11 \%$ des élèves ont plus de 6 ans et sont donc déjà considérés en retard dès leur entrée dans l'enseignement primaire. Les retards s'additionnant au cours du cursus, les taux augmentent ensuite lentement durant le primaire et dépassent $21 \%$ en $5^{\mathrm{e}}$ et $6^{\mathrm{e}}$ années. La figure 1 montre qu'une différenciation des effectifs se manifeste ensuite dès le début du secondaire. Passant de $29 \%$ environ en $1^{\text {re }}$ année à $28 \%$ en $6^{\mathrm{e}}$, la forme générale est marquée par une stabilisation des taux. La situation est différente dans toutes les autres formes du secondaire où, quelle que soit l'année d'études, les taux sont près de deux fois supérieurs à ceux du général. Une première sélection apparaît clairement à l'entame du $1^{\text {er }}$ degré, puisque le taux de retard en $1 \mathrm{~B}$ est de $76 \%$ et passe à $70 \%$ en $2 \mathrm{P}$ pour seulement $29 \%$ en $1 \mathrm{~A}$ et $2 \mathrm{C}$. Une seconde sélection s'effectue au début du $2^{\mathrm{e}}$ degré, hiérarchisant de façon permanente les formes d'enseignement jusqu'à la fin du secondaire. En effet, en $3^{e}$ année, le taux de retard passe de $25 \%$ pour le général à $54 \%$ en technique de transition, $69 \%$ en technique de qualification, $77 \%$ en professionnelle et plus de $90 \%$ dans les CEFA.

Si l'on établit la répartition différenciée des effectifs selon le sexe (figure 2), on observe d'abord un bon équilibre entre garçons et filles durant tout le fondamental et le $1^{\text {er }}$ degré du secondaire général. Comme pour le retard, la différenciation des effectifs augmente ensuite faiblement dans le secondaire, surtout jusque la fin du $2^{\mathrm{e}}$ degré. Globalement, la forme générale est de plus en plus féminine, comptant de $50 \%$ à $55 \%$ de filles de la $2^{\mathrm{e}}$ à la $6^{\mathrm{e}}$ année. Les autres formes sont majoritairement masculines, principalement durant le $1^{\mathrm{er}}$ degré où la proportion de garçons est proche de $58 \%$. Seuls les CEFA et la $7^{e}$ préparatoire à l'enseignement supérieur enregistrent plus de $64 \%$ de garçons et, à l'opposé, le 4 e degré professionnel complémentaire compte plus de $85 \%$ de filles.

Un examen de la figure 3 montre que le processus de différenciation entre Belges et élèves de nationalité étrangère est similaire à celui observé entre filles et garçons et surtout au processus de différenciation selon le retard scolaire. Si la proportion d'élèves de nationalité étrangère en primaire est proche de $10 \%$, moyenne observée en Communauté française, lors du parcours scolaire en secondaire, ce pourcentage se réduit progressivement dans les sections de transition pour se limiter à $5,8 \%$ en $6^{\mathrm{e}}$ générale. À l'opposé, la proportion d'élèves de nationalité étrangère augmente dans les sections de qualification pour atteindre un maximum en professionnelle, principalement au $4^{\mathrm{e}}$ degré, type d'études complémentaires où la proportion d'élèves de nationalité étrangère, principalement des étudiants français, atteint $58 \%$ en $1^{\text {re }}$ année.

De façon très générale, les formes d'enseignement et les années d'études enregistrant le plus d'élèves en retard scolaire sont aussi les formes et les années les plus masculines et celles enregistrant le plus de jeunes de nationalité étrangère. 


\subsection{Répartition différenciée des effectifs de l'enseignement ordinaire selon le retard des élèves en 2005-2006}

FONDAMENTAL (MATERNEL ET PRIMAIRE)

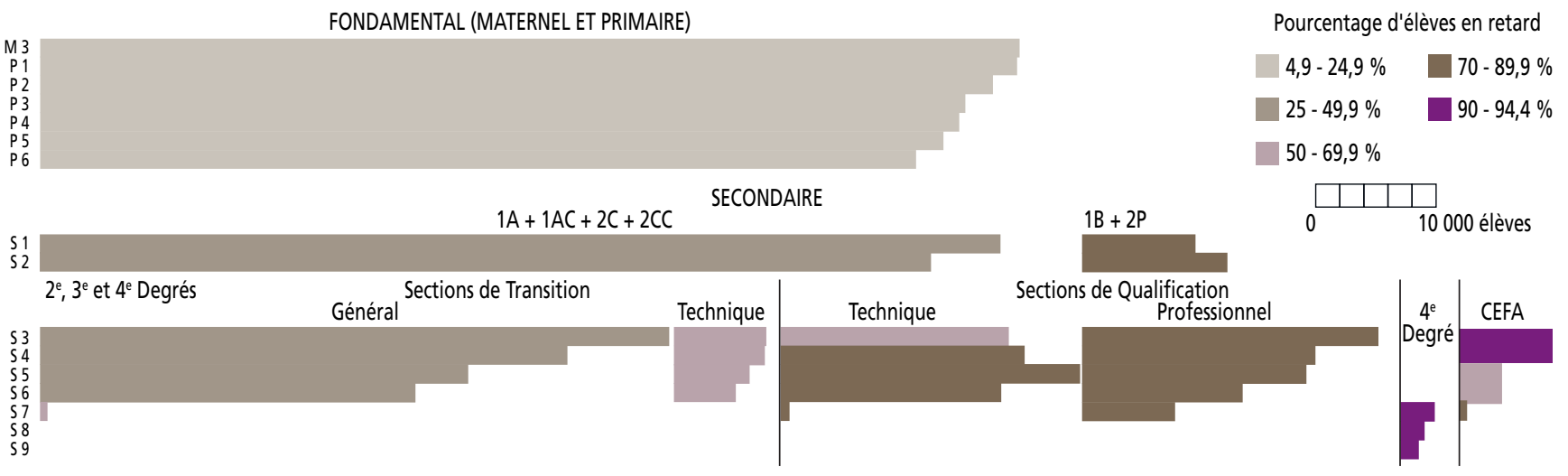

\subsection{Répartition différenciée des effectifs de l'enseignement ordinaire selon le sexe en 2005-2006}

\section{FONDAMENTAL (MATERNEL ET PRIMAIRE)}

$M 3$
$P 1$
$P 2$
$P 3$
$P 3$
$P 4$
$P 5$
$P 6$

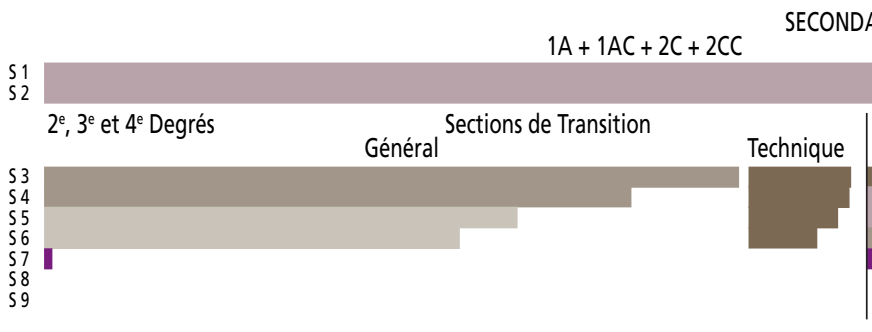

Pourcentage de garçons

$11,4-46,9 \% \square 53-62,9 \%$

$47-49,9 \%$

$63-79,2 \%$

$50-52,9 \%$

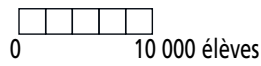

\subsection{Répartition différenciée des effectifs de l'enseignement ordinaire selon la nationalité en 2005-2006}

FONDAMENTAL (MATERNEL ET PRIMAIRE)
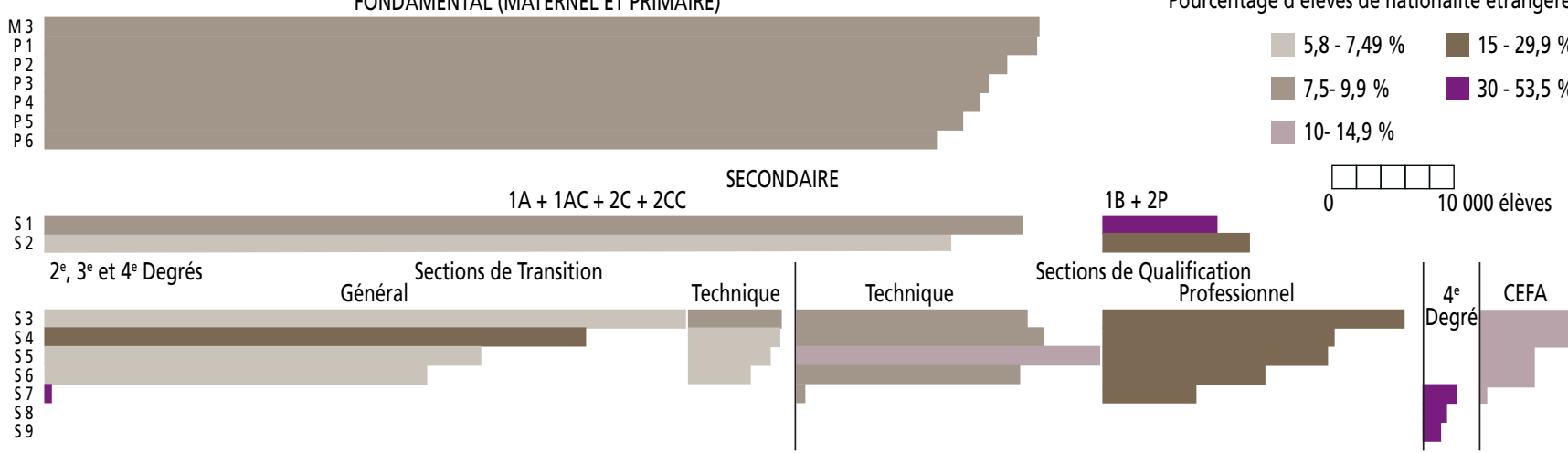

Pour chaque année d'études, afin de visualiser le poids relatif de celle-ci, la longueur de la barre horizontale du graphique est proportionnelle à l'effectif des élèves. La couleur indique la catégorie de valeurs des trois variables retenues : le retard, le sexe et la nationalité. Ainsi, dans l'ensemble des $2^{\mathrm{e}}$ et $3^{\mathrm{e}}$ degrés du secondaire professionnel, le nombre d'élèves en retard est compris entre 70 et $90 \%$ du total.
Pourcentage d'élèves de nationalité étrangère

$5,8-7,49 \% \square 15-29,9 \%$

$7,5-9,9 \%$

$30-53,5 \%$

$10-14,9 \%$ 


\section{Population scolaire, par réseau, dans l'enseignement fondamental et secondaire}

Depuis 1994-1995, la population scolaire de l'enseignement fondamental et secondaire se répartit à parts quasi égales entre, d'une part, les réseaux officiels (enseignement organisé par la Communauté française et enseignement officiel subventionné) et, d'autre part, le réseau libre subventionné. Cette répartition n'est pas identique à tous les niveaux d'enseignement. Ainsi, dans les niveaux maternel et primaire, la part majoritaire du réseau officiel subventionné progresse alors que dans le secondaire, la situation et la tendance sont inversées au profit du réseau libre subventionné.

'enseignement est une compétence des trois Communautés de Belgique. La Communauté française organise un enseignement et subventionne l'enseignement organisé par différents pouvoirs organisateurs. Ainsi, les pouvoirs organisateurs sont d'une part, la Communauté elle-même, des personnes morales de droit public (les provinces, les communes et la Cocof) qui organisent l'enseignement officiel et d'autre part, des personnes morales de droit privé (associations, congrégations religieuses), qui organisent l'enseignement libre, confessionnel ou non confessionnel. Ce mode d'organisation découle de la liberté d'enseignement inscrite dans la Constitution qui garantit également aux parents le libre choix de l'école dans laquelle ils scolarisent leur enfant.

La figure 1 présente l'évolution de la population scolaire et sa répartition par réseau dans l'enseignement fondamental et secondaire, ordinaire et spécialisé, de 1994-1995 à 2005-2006. En 2005-2006, l'enseignement libre subventionné accueille $48,7 \%$ de la population scolaire, l'enseignement officiel $51,3 \%$ dont 35,8 \% dans l'officiel subventionné et $15,5 \%$ dans l'enseignement organisé par la Communauté française. Sur les 12 années présentées, la population scolaire de l'enseignement fondamental et secondaire a augmenté de 3,2 \% et la répartition des élèves dans les réseaux est restée stable. Au sein de I'enseignement officiel, on note toutefois une baisse de 1,5 point dans I'enseignement organisé par la Communauté française et, dans le même temps, une augmentation de 1,4 point dans le réseau officiel subventionné.

Dans l'enseignement fondamental ordinaire (figure 2), l'enseignement officiel subventionné scolarise plus de la moitié des élèves : dans le niveau maternel, ses effectifs ont augmenté de 3,5 points sur les 12 années scolaires considérées, pour atteindre 54,2 \% en 2005-2006 et dans le primaire, $48,8 \%$ des élèves y sont scolarisés, après une augmentation de 4,1 points en 12 ans. Parallèlement, l'enseignement libre subventionné a connu une diminution de 1,5 point dans le maternel et de 2,4 points dans le primaire et l'enseignement organisé par la Communauté française, une diminution de 2,0 points dans le maternel et de 1,7 point dans le primaire.

Dans le secondaire ordinaire de plein exercice, l'enseignement libre subventionné accueille plus de la moitié des élèves : 59,5 \% en 2005-2006 (augmentation de 2,9 points depuis 1994-1995). En 2005-2006, I'enseignement officiel subventionné rassemble $16,2 \%$ de la population scolaire (diminution de 0,9 point sur 12 ans) et l'enseignement organisé par la Communauté française 24,3 \% (diminution de 2,0 points dans le même temps). Ces proportions diffèrent lorsqu'on considère uniquement l'enseignement secondaire qualifiant : I'enseignement libre accueille 54,1\% des élèves, l'officiel subventionné $26,2 \%$ et l'enseignement organisé par la Communauté française $19,7 \%$; l'enseignement officiel subventionné prenant ainsi une part plus importante dans l'enseignement qualifiant que dans l'ensemble de l'enseignement secondaire.

L'enseignement secondaire ordinaire en alternance (figure 3) a vu ses effectifs augmenter de plus de $65 \%$ sur 9 ans. L'enseignement libre subventionné, qui rassemblait $50,5 \%$ de la population scolaire des CEFA en 1997-1998 n'en compte plus que $47,4 \%$ en $2005-2006$, soit une diminution de 3,1 points; dans le même temps, la population scolaire de l'enseignement officiel subventionné augmente de 10,1 points et l'enseignement organisé par la Communauté française diminue de 6,0 points. On remarque que c'est en 2005-2006 que l'enseignement officiel subventionné a pris une part plus importante, au détriment de l'enseignement organisé par la Communauté française.

Dans l'enseignement spécialisé (figure 4), la population scolaire a augmenté de $6,7 \%$ en 12 ans et la répartition des élèves entre les réseaux est restée pratiquement stable : $48,3 \%$ dans le réseau libre (diminution de 0,4 point), $26,6 \%$ dans l'officiel subventionné (moins 0,8 point) et $25,1 \%$ dans le réseau organisé par la Communauté française (plus 1,2 point).

En résumé, la répartition des effectifs scolaires entre les réseaux se caractérise comme suit :

- pour l'ensemble de l'enseignement fondamental et secondaire, le réseau de la Communauté française compte 1 élève sur 6 , l'officiel subventionné 1 sur 3 et le libre subventionné 1 sur 2 ;

- le réseau libre subventionné scolarise près de 3 élèves sur 5 du secondaire ordinaire ;

- le réseau officiel subventionné accueille plus de la moitié des élèves du fondamental et se distingue, au niveau secondaire ordinaire de plein exercice, par une proportion d'élèves plus importante dans l'enseignement de qualification $(26,2 \%)$ que dans l'ensemble de l'enseignement secondaire $(16,2 \%)$;

- I'enseignement organisé par la Communauté française accueille, dans l'enseignement secondaire ordinaire de plein exercice, proportionnellement près de 3 fois plus d'élèves qu'au fondamental ordinaire et ce réseau est plus particulièrement représenté dans l'enseignement spécialisé. 
6.1 Évolution de la population scolaire et de sa répartition par réseau dans l'enseignement fondamental et secondaire de 1994-1995 à 2005-2006

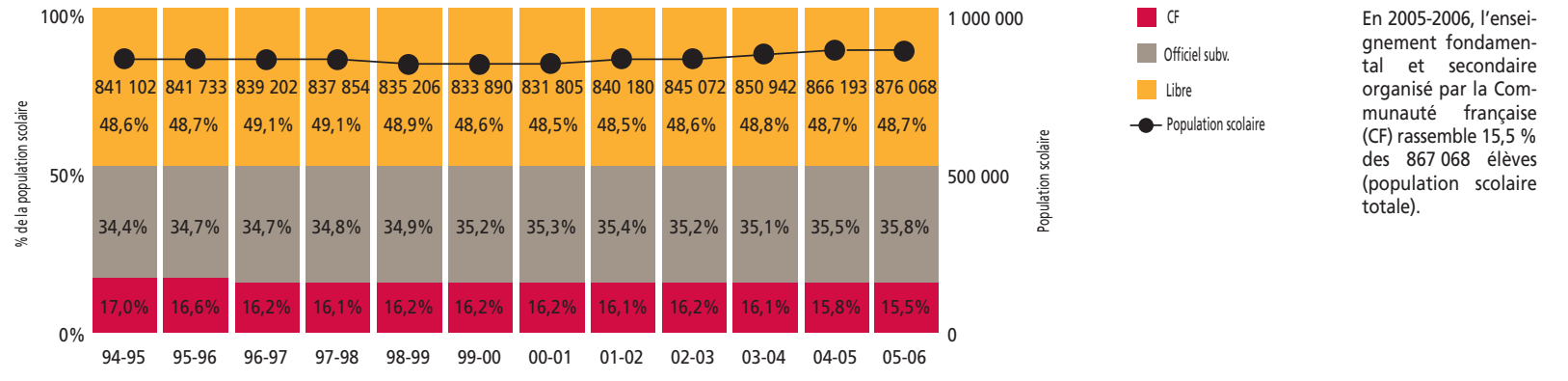

6.2 Évolution de la population scolaire et de sa répartition par réseau dans l'enseignement ordinaire de plein exercice de 1994-1995 à 2005-2006
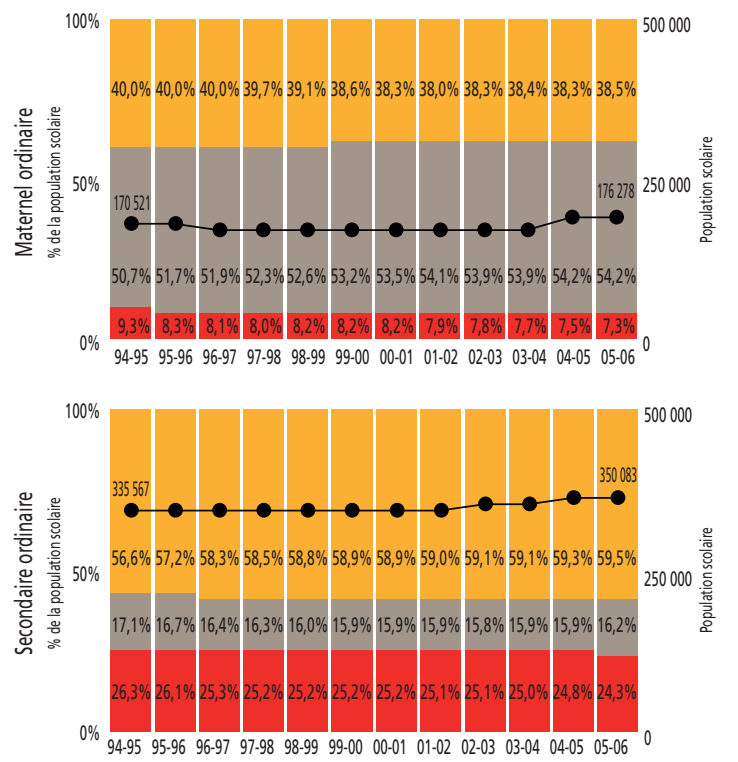

$100 \%$

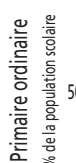

$\frac{\sum_{2}}{\frac{1}{2}}$

,

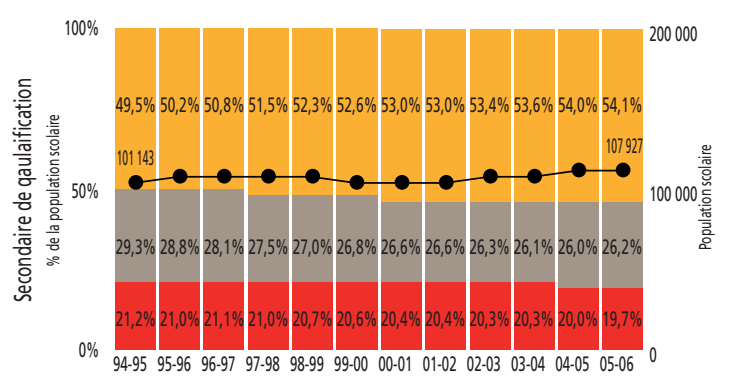

$\square$ CF

Officiel subv.

Libre

- Population scolaire

En 2005-2006, I'enseignement primaire ordinaire organisé par le réseau officiel subventionné rassemble $48,8 \%$ des 301932 élèves de ce niveau.
6.3 Évolution de la population scolaire et de sa répartition par réseau dans l'enseignement secondaire ordinaire en altemance (CEFA) de 1997-1998 à 2005-2006

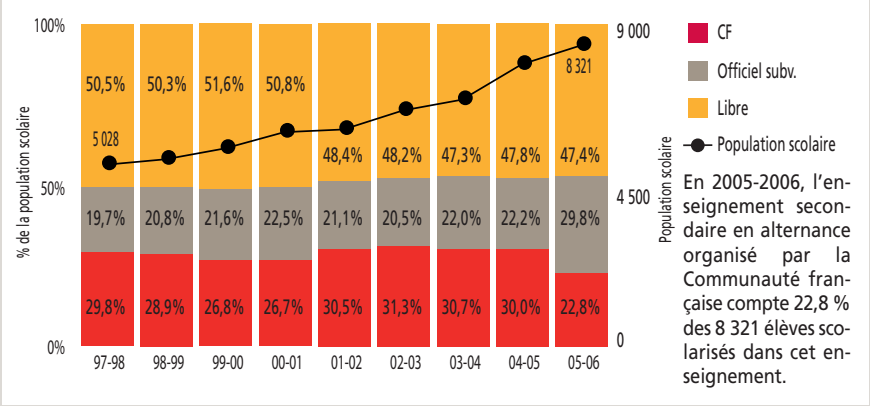

6.4 Évolution de la population scolaire et de sa répartition par réseau dans l'enseignement spécialisé de 1994-1995 à 2005-2006

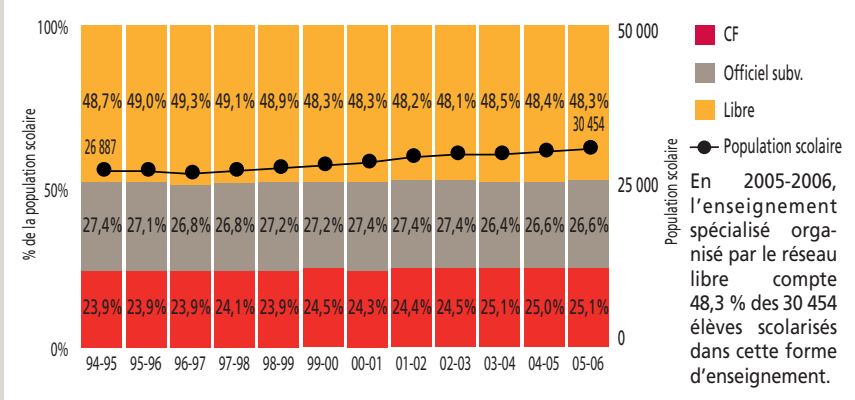


Une disparité se manifeste dans les niveaux, formes et années d'études lorsqu'ils sont mis en relation avec le niveau socioéconomique du quartier de résidence de l'élève.

La répartition différenciée des effectifs scolaires en Communauté française apparaît très tôt dans le parcours scolaire et se poursuit, en s'accentuant, tout au long du parcours dans l'enseignement obligatoire.

'étude porte sur le public de l'enseignement fondamental et secondaire, en 2005-2006, sur la base des caractéristiques socioéconomiques des quartiers où vivent les élèves.

Le niveau socioéconomique est abordé à partir d'indices déterminés par secteur ou quartier statistique ${ }^{1}$, indices calculés et actualisés selon 11 critères précis ${ }^{2}$. La valeur moyenne des indices est fixée à 0 . Un indice est ensuite attribué à chaque élève en fonction de son quartier de résidence ${ }^{3}$. Cette valeur appelée " indice socioéconomique » est par ailleurs le point de départ de la détermination des écoles en discrimination positive basée sur un classement des implantations scolaires en lien avec la moyenne des indices socioéconomiques des élèves qui les fréquentent.

La figure 1 présente les indices socioéconomiques moyens des quartiers où résident les élèves en fonction des niveaux et formes d'enseignement fréquentés. $\mathrm{Si}$, dans l'enseignement ordinaire, la valeur de I'indice est proche de 0 , l'enseignement spécialisé, quant à lui, se positionne, tous niveaux confondus, à l'indice $-0,43$. Un écart comparable se marque entre l'enseignement secondaire ordinaire de plein exercice et l'enseignement secondaire en alternance.

La figure 2 présente les indices socioéconomiques moyens des quartiers où résident les élèves de l'enseignement secondaire ordinaire en fonction des années d'études et des formes d'enseignement suivies ${ }^{4}$. Une disparité importante entre les formes de l'enseignement secondaire apparaît. Elle commence dès l'entrée dans le secondaire avec un écart de plus de 0,6 entre les indices moyens du $1^{\mathrm{er}}$ degré « commun » $(1 \mathrm{~A}-2 \mathrm{C})$ et du $1^{\text {er }}$ degré « différencié » (1B-2P), qui accueille une majorité d'élèves n'ayant pas obtenu de CEB (certificat d'études de base) en primaire. Cette dispersion s'accentue dans les $2^{\mathrm{e}}$ et $3^{\mathrm{e}}$ degrés où le niveau socioéconomique moyen pour les élèves fréquentant la forme professionnelle (de la $3 \mathrm{P}$ à la $6 \mathrm{P}$ ) est de $-0,33$, pour la forme technique de qualification (de la 3TQ à la 6TQ) de -0,06; pour la forme technique de transition (de la 3TT à la 6TT), l'indice moyen passe à $+0,21$ et enfin pour la forme générale (de la $3 G$ à la $6 G$ ), il atteint $+0,31$. Ainsi la $2 C$, à l'issue de laquelle intervient l'orientation, agit véritablement comme " gare de triage » en fonction du niveau socioéconomique des élèves pour leur choix en troisième année.

Comme le montre la figure 2, dans chaque forme d'enseignement, I'indice socioéconomique moyen progresse avec l'année d'études. Cela peut s'expliquer par la sortie, durant le parcours scolaire, d'élèves socioéconomiquement défavorisés, ou encore par une possible augmentation de la moyenne de l'indice dans d'autres formes d'enseignement, si des élèves socioéconomiquement plus favorisés y sont réorientés.

En conclusion, considérant le niveau socioéconomique du lieu de résidence des élèves, la répartition différenciée des effectifs scolaires en Communauté française est un fait : elle apparaît très tôt dans le parcours scolaire et se poursuit, en s'accentuant, tout au long du parcours dans l'enseignement obligatoire.

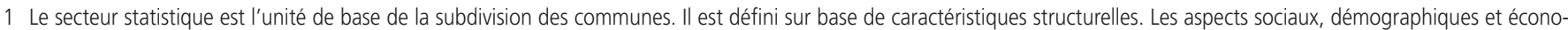
miques, généralement observables dans le bâti, sont déterminants. Un quartier ou secteur statistique est la plus petite entité administrative pour laquelle des données socioéconomiques et administratives sont disponibles.

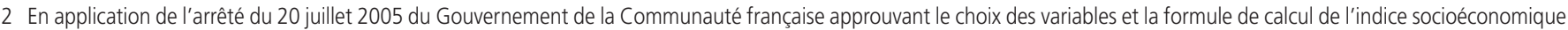
de chaque secteur statistique, pour chaque quartier (9.310 secteurs statistiques), l'indice composite socioéconomique est calculé sur la base des 11 variables socioéconomiques suivantes (avec indication de la dernière année disponible par variable) : (1) revenu moyen par habitant en euros (2001); (2) revenu médian par ménage en euros (2001); (3) part des personnes ayant terminé leurs études qui disposent au moins d'un diplôme de l'enseignement secondaire supérieur parmi les personnes ayant achevé leurs études (2001); (4) part des ménages avec enfant dont une personne au moins dispose d'un diplôme de l'enseignement secondaire supérieur parmi les ménages avec enfants (2001); (5) taux de chômage : ensemble des demandeurs d'emploi (y compris non rémunérés) parmi les personnes présentes sur le marché du travail (2001); (6) taux d'activité des femmes : ensemble des femmes occupant un emploi / femmes de 18 à 60 ans ayant terminé leurs études (2001); (7) taux de bénéficiaires du revenu mensuel minimum garanti (1998); (8) part des professions de bas standing : personnes exerçant une profession de bas standing / ensemble des personnes actives dont on connaît la profession (1991); (9) part des professions de haut standing : personnes exerçant une profession de haut standing / ensemble des personnes actives dont on connaît la profession (1991); (10) part des logements disposant du grand confort : logements disposant d'une cuisine d'au moins $4 \mathrm{~m}^{2}$, d'un téléphone, de l'eau courante, d'une salle de bain, du chauffage central et d'une voiture / total des logements (2001) ; (11) nombre de pièces pour 100 habitants : (nombre total de pièces x 100) / population (2001).

II convient de souligner que la formule de calcul prend en compte les dernières données statistiques disponibles, et ce pour les seuls secteurs statistiques de la région bilingue de Bruxelles-Capitale et de la région de langue française, comme le prescrit l'article 4 du décret du 27 mars 2002.

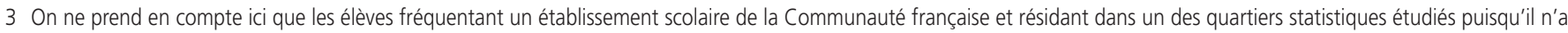
pas été possible d'attribuer un indice socioéconomique aux élèves résidant à l'étranger ou en région flamande.

4 II n'a pas été possible d'inclure les données de l'enseignement secondaire spécialisé et en alternance, car elles ne sont pas disponibles par année d'études. 
7.1 Indice socioéconomique moyen des quartiers où résident les élèves des différents niveaux et formes de l'enseignement fondamental et secondaire (de plein exercice et en alternance) - Année scolaire 2005-2006

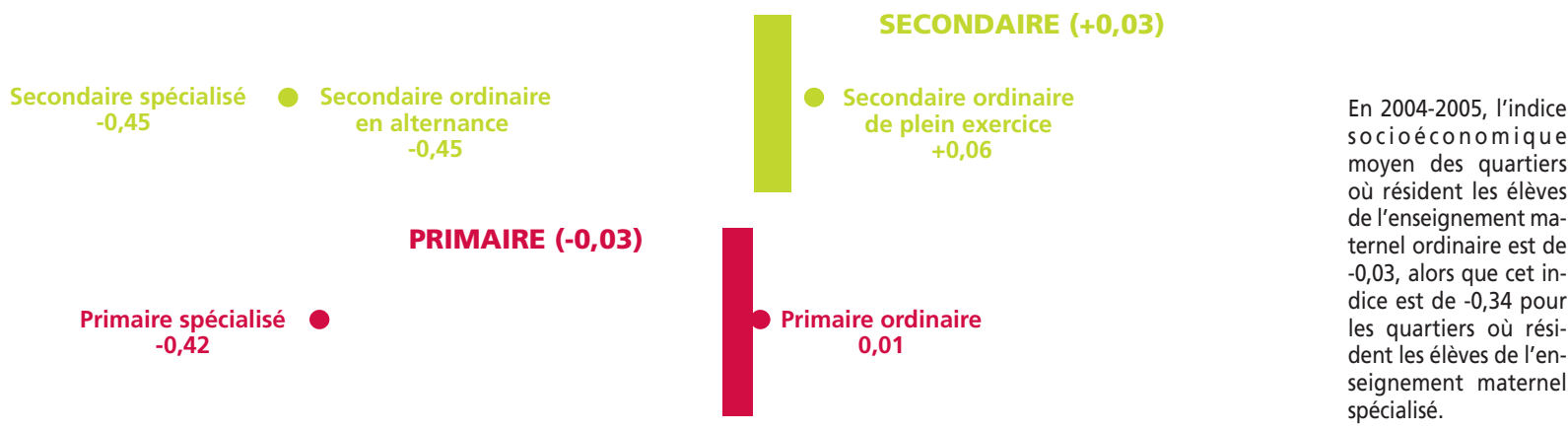

PRIMAIRE $(-0,03)$

Primaire spécialisé

$-0,42$

Primaire ordinaire

0,01

2004-2005, l'indice socioéconomique moyen des quartiers où résident les élèves de l'enseignement maternel ordinaire est de $-0,03$, alors que cet indice est de $-0,34$ pou les quartiers où résident les élèves de l'enseignement maternel spécialisé.

\section{MATERNEL $(-0,04)$}

Maternel spécialisé $-0,34$
0,2

Indices de milieux socioéconomiquement favorisés

7.2 Indice socioéconomique moyen des quartiers où résident les élèves des différentes années d'études et formes de l'enseignement secondaire ordinaire de plein exercice - Année scolaire 2005-2006
6e année
$3^{e}$ année
$5^{e}$ année
$4^{\mathrm{e}}$ année
$2^{\mathrm{e}}$ année
$1^{\text {re }}$ année
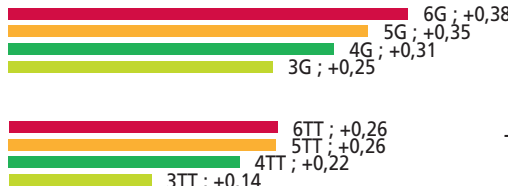

Technique et artistique de transition

(TT) $(+0,21)$

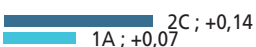

1er degré « commun »

Technique et artistique de qualification (TQ) $(-0,06)$

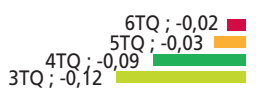

Professionnel

(P) $(-0,33)$

4 4P $;-0,41^{5 P} ;-0,28 ;-0,22$

$1^{\text {er }}$ degré « différencié » $(-0,51)$

$1 \mathrm{~B} ;-0,68$

$2 \mathrm{P} ;-0,38$

$-0,8$

$-0,6$

$-0,4$

$-0,2$

4 Indices de milieux socioéconomiquement défavorisés

Indice socioéconomique moyen

Indices de milieux socioéconomiquement favorisés
$(+0,10)$

Général

(G) $(+0,31)$

En 2005-2006, l'indice socioéconomique moyen des quartiers où résident les élèves de 1B est de -0,68 alors que cet indice atteint + 0,38 pour les quartiers où résident les élèves de 6G. 
En 2005-2006, 378 implantations de l'enseignement fondamental et 127 de l'enseignement secondaire étaient visées par la mise en œuvre de discriminations positives en Communauté française.

C'est dans la province du Hainaut que le nombre d'implantations en discrimination positive est le plus important et, pour une population scolaire quatre fois moins importante, Bruxelles concentre quasi autant d'élèves en discrimination positive que la Wallonie dans son ensemble.

Un élève sur huit est concerné par la mise en œuvre de discriminations positives en Communauté française, un élève sur dix en Wallonie, et un élève sur quatre à Bruxelles.

La répartition des effectifs scolaires en discrimination positive par réseaux présente également des différences significatives.

a discrimination positive, définie par le décret du 30.06.1998, permet d'attribuer des moyens complémentaires au bénéfice d'établissements ou d'implantations d'enseignement ordinaire fondamental et secondaire, organisés ou subventionnés par la Communauté française de Belgique sur la base de critères sociaux, économiques, culturels et pédagogiques ${ }^{1}$. Le décret vise à assurer à tous les élèves des chances égales d'émancipation sociale, et donc à rencontrer une des quatre missions prioritaires de l'enseignement.

La détermination des écoles en discrimination positive est réalisée à partir d'un indice socio-économique calculé pour chaque implantation scolaire ${ }^{2}$. Tous les trois ans, les implantations sont classées par ordre croissant, des moins favorisées aux plus favorisées. Sont retenues pour bénéficier des discriminations positives les implantations les moins favorisées jusqu'à atteindre $12,5 \%$ des effectifs scolaires dans l'enseignement fondamental et $13,5 \%$ dans l'enseignement secondaire.

La figure 1 propose une cartographie des implantations fondamentales et secondaires en discrimination positive. Celles qui bénéficient du dispositif de la discrimination positive se concentrent principalement sur 3 zones géographiques: Bruxelles, la province du Hainaut et la province de Liège. A Bruxelles, 90 implantations fondamentales et 49 implantations secondaires sont visées par la discrimination positive. Les implantations bruxelloises représentent près d'un quart des implantations en discrimination positive au niveau fondamental. Dans le Hainaut, les implantations en discrimination positive sont au nombre de 170 pour le fondamental et d'une soixantaine pour le secondaire. En ce qui concerne Liège, 98 implantations fondamentales et 16 implantations secondaires sont concernées par la discrimination positive. Quant à la province de Namur, il y a 16 implantations fondamentales et 2 implantations secondaires. Une implantation fondamentale est visée par la discrimination positive dans le Brabant wallon ; 3 implantations sont concernées dans le Luxembourg pour l'année scolaire 2005-2006.

La figure 2 présente la répartition des effectifs scolaires en discrimination positive par niveau d'enseignement en Communauté française, effectifs partagés entre la Région bruxelloise et la Région wallonne. Le premier diagramme montre que le pourcentage des effectifs scolaires en discrimination positive est égal à 12,3\% pour le fondamental et $13,1 \%$ la population scolaire de la Communauté française. Le deuxième et le troisième diagramme révèlent des disparités régionales. En Région bruxelloise, l'effectif scolaire en discrimination positive représente $23,7 \%$ dans le fondamental et $27,5 \%$ dans le secondaire. En Région wallonne, l'effectif en discrimination positive représente $9,2 \%$ dans le fondamental et 9,4\% dans le secondaire ${ }^{3}$.

La figure 3 distingue les effectifs scolaires en discrimination positive par réseau d'enseignement. Les trois premiers diagrammes montrent la répartition des effectifs scolaires au niveau fondamental. Pour l'ensemble de la Communauté française, les 12,3 \% d'élèves en discrimination positive se répartissent à raison d'1 $1 \%$ dans le réseau de la Communauté française, $7 \%$ dans le réseau officiel subventionné et $4 \%$ dans le réseau libre subventionné. Ainsi, 1 élève sur 10 est en discrimination positive dans le réseau de la Communauté française, près de 1 élève sur 7 dans le réseau officiel subventionné et près de 1 sur 11 dans le réseau libre subventionné. En Région bruxelloise, ces rapports sont respectivement de 1 élève sur 2, 1 élève sur 4 et 1 élève sur 5 , tandis qu'en Région wallonne, ils sont de 1 élève sur 26, 1 élève sur 9 et 1 élève sur 12 . Les trois derniers diagrammes présentent la répartition dans l'enseignement secondaire. Pour l'ensemble de la Communauté française, les $13 \%$ de l'effectif scolaire en discrimination positive se répartissent entre les réseaux de la manière suivante : $3,4 \%$ dans le réseau de la Communauté française, 4,3\% dans celui de l'officiel subventionné et $5,4 \%$ dans le réseau libre subventionné. Les rapports au sein des réseaux sont respectivement de 1 élève en discrimination positive sur 7, 1 élève sur 4 et 1 élève sur 11. En Région bruxelloise, ces rapports sont de 1 élève sur 3 pour le réseau de la Communauté française et l'officiel subventionné, et de 1 élève sur 5 dans le réseau libre subventionné. En Région wallonne, 1 élève sur 11 est en discrimination positive dans le réseau de la Communauté française, 1 sur 4 dans le réseau officiel subventionné et 1 sur 16 dans le réseau libre subventionné.

Finalement, trois points sont à retenir : les implantations en discrimination positive se situent principalement à Bruxelles, à Liège et dans le Hainaut ; à Bruxelles, les élèves en discrimination positive représentent un quart de l'effectif scolaire de cette région ; la proportion des élèves en discrimination positive est plus importante dans le réseau officiel subventionné et dans le réseau de la Communauté française que dans le réseau libre subventionné.

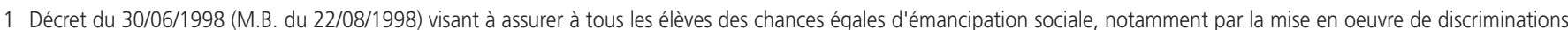
positives.

2 Voir indicateur 7 - Disparités socioéconomiques dans l'enseignement fondamental et secondaire.

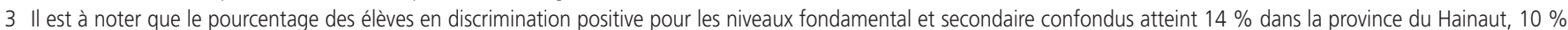
dans la province de Liège, 1,3\% dans la province de Namur et 0,3\% dans la province du Luxembourg. 

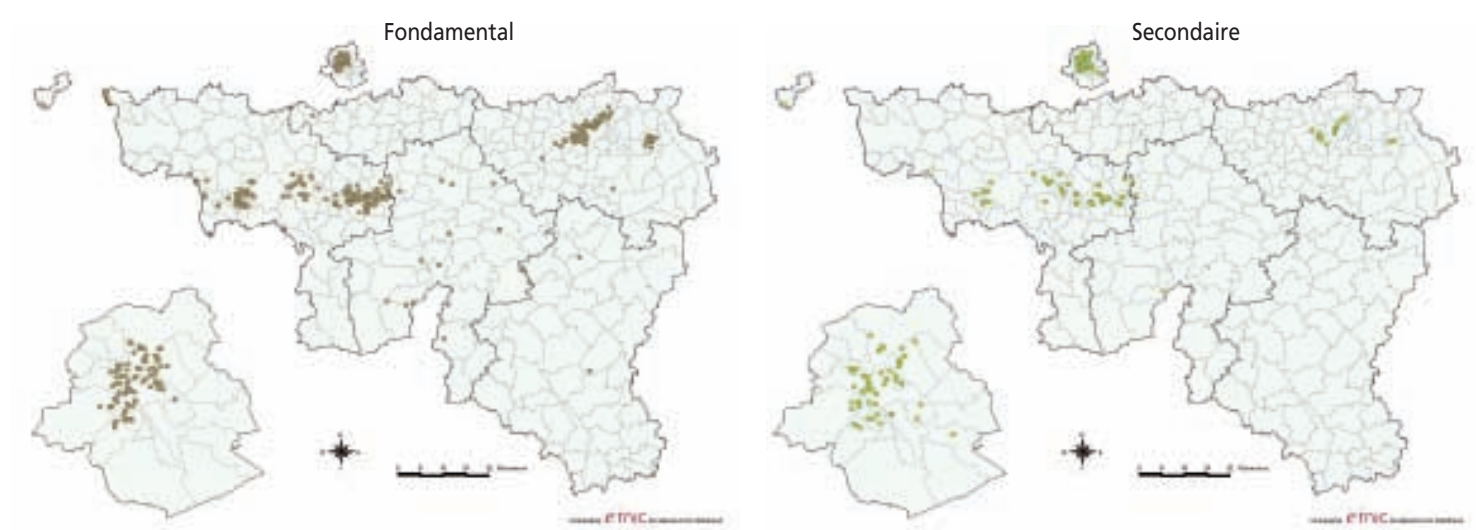

En 2005-2006, les écoles d'enseignement fondamental en discrimination positive, tous réseaux confondus, se situent principalement dans les provinces du Hainaut, de Liège et dans la Région bruxelloise.

8.2 Répartition des effectifs scolaires de l'enseignement fondamental et secondaire ordinaire en discrimination positive en Communauté française, effectifs partagés entre la Région bruxelloise et wallonne en 2005-2006

Communauté française

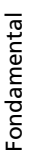

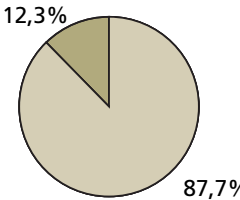

$87,7 \%$

음

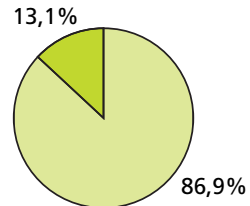

Région bruxelloise

$23,7 \%$

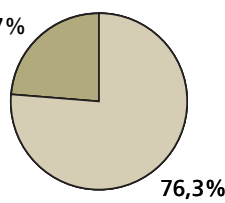

$27,5 \%$

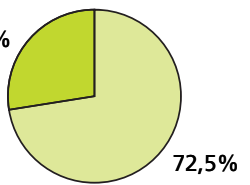

Région Wallonn
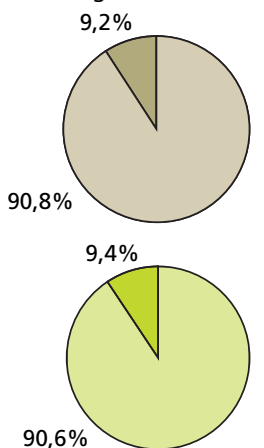

Non D+ D+

Fondamental

Secondaire

En 2005-2006, les élèves en discrimination positive représentent $13,1 \%$ de I'effectif de l'enseignement secondaire ordinaire de plein exercice, en Communauté française.

\subsection{Répartition des effectifs scolaires en discrimination positive par réseaux en 2005-2006}

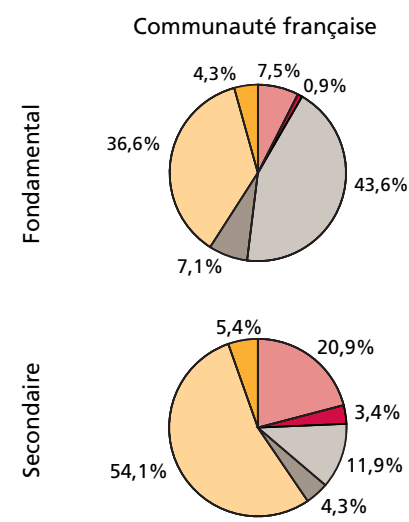

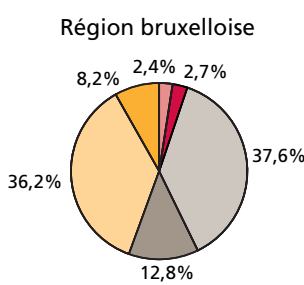

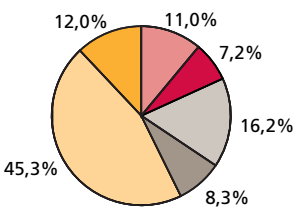

$36,7 \%$

\section{Région Wallonne}

$3,2 \% \quad 8,9 \%$

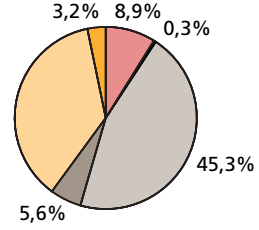

$56,4 \%$

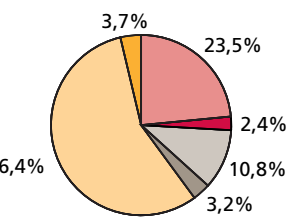

Non $\mathrm{D}+\quad \mathrm{D}+$

Réseau de la

Communauté française

Réseau officiel

subventionné

Réseau libre subventionné

En 2005-2006, parmi les effectifs de l'enseignement fondamental ordinaire, en Région wallonne, $6 \%$ fréquentent des établissements en discrimination positive dans le réseau officiel subventionné. 
Entre 6 ans et 12 ans, la proportion de jeunes inscrits dans l'enseignement spécialisé par rapport à la population totale de l'enseignement obligatoire quadruple.

A partir de 13 ans, on assiste à un repli substantiel de la proportion de jeunes inscrits dans l'enseignement spécialisé, suite à une transition, essentiellement des jeunes inscrits dans le type 8, vers l'enseignement secondaire ordinaire.

Le taux de fréquentation de l'enseignement spécialisé est très sensible au niveau socioéconomique de l'élève : il est de 1,5\% chez les $10 \%$ des élèves résidant dans les quartiers les plus favorisés et s'élève à $5,9 \%$ chez les $10 \%$ des élèves résidant dans les quartiers les plus défavorisés.

'et indicateur montre le rapport entre le nombre d'élèves inscrits dans

I'enseignement spécialisé et le nombre total d'élèves inscrits dans I'enseignement maternel et obligatoire ${ }^{1}$ selon l'âge (figure 1) et le niveau socioéconomique du quartier de résidence de l'élève (figure 2 ). Seuls les élèves domiciliés en Belgique sont pris en considération dans cet indicateur.

La figure 1 met en évidence, pour chaque âge, la proportion de jeunes inscrits dans l'enseignement spécialisé par rapport à la population totale de l'enseignement maternel et obligatoire, en distinguant les types $^{2} \mathrm{~d}^{\prime}$ enseignement spécialisé fréquentés. Cette figure permet ainsi de visualiser à quel moment du cursus scolaire l'enseignement spécialisé accueille la plus forte proportion d'élèves.

C'est à l'âge de 12 ans que la proportion d'élèves inscrits dans l'enseignement spécialisé est la plus forte : $7 \%$ chez les garçons et 4,3\% chez les filles. A l'âge de 6 ans, ces mêmes proportions sont respectivement de $1,7 \%$ et $0,9 \%$. La croissance de la proportion d'élèves dans le spécialisé au fil des âges se produit, pour l'essentiel, dans deux types d'enseignement, le type 8 (troubles instrumentaux) et le type 1 (arriération mentale légère), où la proportion de jeunes inscrits passe de $0,7 \%$ chez les garçons de 6 ans à 4,9\% chez les garçons de 12 ans et de 0,3\% à $3,4 \%$ chez les filles du même âge.

A partir de 13 ans, on assiste à un repli substantiel de la proportion de jeunes inscrits dans l'enseignement spécialisé ; chez les garçons, ils ne sont plus que 5,1\% et, chez les filles, 3,3 \% à fréquenter cet enseignement. D'une manière plus précise, cette chute est due à une forte diminution du taux de fréquentation dans le type 8 (troubles instrumentaux), légèrement compensée par une hausse du taux de fréquentation dans le type 1 (arriération mentale légère). La raison est qu'entre 12 et 13 ans, une partie importante des élèves de l'enseignement spécialisé passent du niveau primaire au niveau secondaire et que ce passage, pour les jeunes inscrits dans le type 8 (troubles instrumentaux), se traduit soit par un transfert vers le type 1 (arriération mentale légère), le secondaire spécialisé n'organisant pas le type 8 , soit par un transfert vers l'enseignement secondaire ordinaire.

Pour réaliser la figure 2 , la population de l'enseignement maternel et obligatoire de la Communauté française a tout d'abord été répartie en déciles par niveau socioéconomique (du quartier de résidence de l'élève) croissant. Dans chaque classe de population, la proportion d'élèves inscrits dans l'enseignement spécialisé a ensuite été calculée, en distinguant les types d'enseignement fréquentés. La figure 2 montre combien le taux de fréquentation de l'enseignement spécialisé est sensible au niveau socioéconomique de l'élève (capté au travers de l'indice socioéconomique de son quartier de résidence).

Pour les $10 \%$ des élèves résidant dans les quartiers les plus favorisés (niveau 10 de la figure 2), la proportion de jeunes inscrits dans l'enseignement spécialisé est de 1,8\% chez les garçons et de 1,2\% chez les filles. A l'opposé, pour les $10 \%$ des élèves résidant dans les quartiers les plus défavorisés (niveau 1 de la figure 2), le taux de fréquentation de l'enseignement spécialisé est de 7,3\% chez les garçons et de 4,5\% chez les filles, soit quatre fois supérieur.

L'écart des taux de fréquentation de l'enseignement spécialisé en fonction du niveau socioéconomique est surtout accentué dans le type 1 (arriération mentale légère). Dans la classe de population au niveau socioéconomique le plus élevé, la proportion de jeunes inscrits dans le type 1 est ainsi de 0,3\% (chez les garçons et chez les filles) tandis que dans la classe de population au niveau socioéconomique le plus bas, le taux de fréquentation du type 1 est de 2,9 \% chez les garçons et de 2,1\% chez les filles, soit huit fois supérieur.

\section{Glossaire}

Types d'enseignement spécialisé :

Type 1 : arriération mentale légère

Type 2 : arriération mentale modérée ou sévère

Type 3 : troubles caractériels et/ou de personnalité

Type 4 : déficiences physiques

Type 5 : maladies

Type 6 : déficiences visuelles

Type 7 : déficiences auditives

Type 8 : troubles instrumentaux

\footnotetext{
1 Enseignement secondaire en alternance (CEFA) inclus.

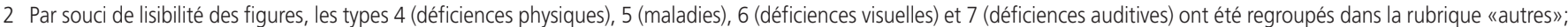
leurs effectifs cumulés représentent moins de $10 \%$ du total des effectifs de l'enseignement spécialisé.
} 
9.1 Proportion d'élèves inscrits dans l'enseignement spécialisé par rapport à la population totale de l'enseignement maternel et obligatoire, selon l'âge et le sexe - Année scolaire 2005-2006

Arriération mentale légère Arriération mentale modérée ou sévère

Garçons

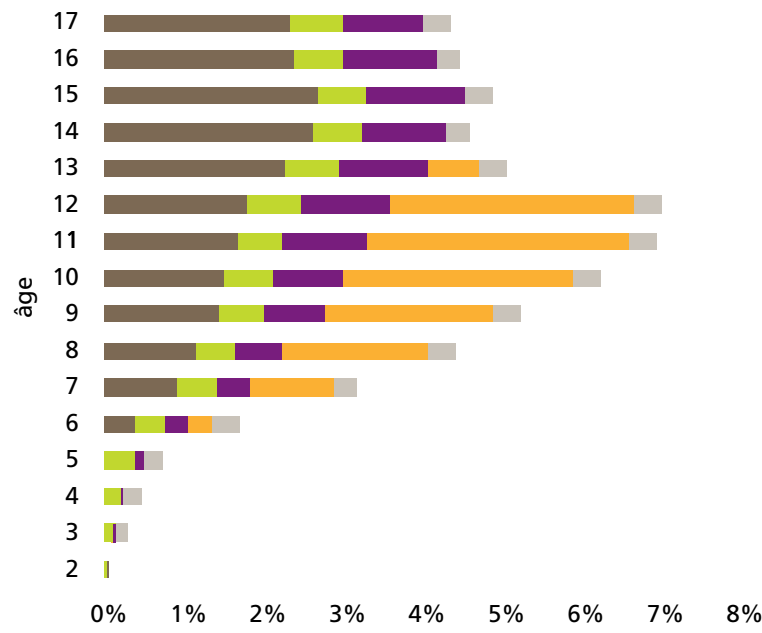

Troubles caractériels et/ou de personnalité

Troubles instrumentaux

Autres

$7 \%$ des garçons et 4,3\% des filles âgés de 12 ans sont inscrits dans l'enseignement spécialisé (tous niveaux d'enseignement confondus).

9.2 Proportion d'élèves inscrits dans l'enseignement spécialisé par rapport à la population totale de l'enseignement maternel et obligatoire, selon le niveau socioéconomique du quartier de résidence et le sexe - Année scolaire 2005-2006

Arriération mentale légère Arriération mentale modérée ou sévère

Garçons

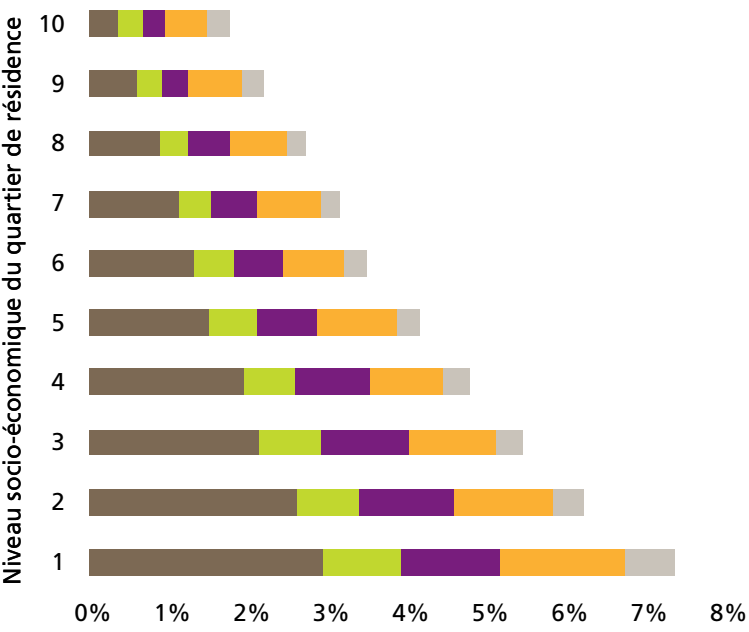

Troubles caractériels et/ou de personnalité

Troubles instrumentaux

Autres

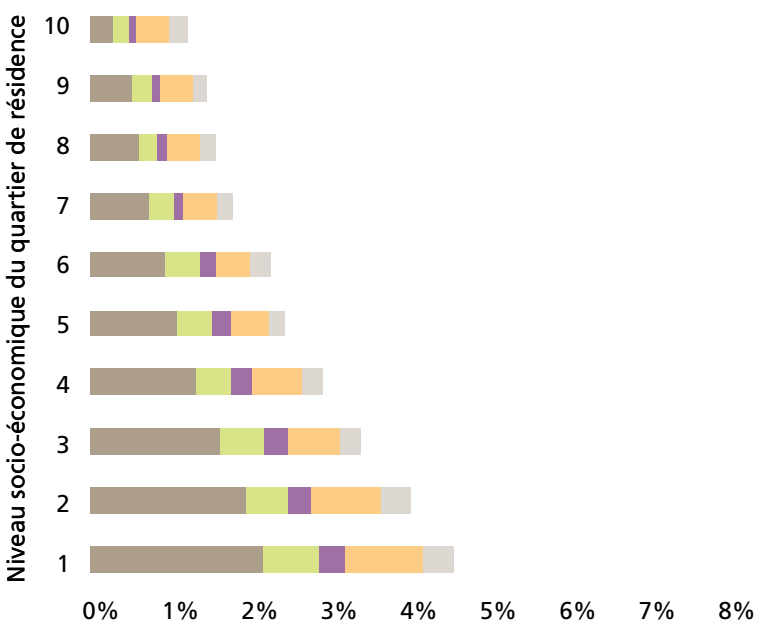

Au niveau 10 les $10 \%$ des élèves résidant dans les quartiers les plus favorisés, la proportion de jeunes inscrits dans l'enseignement spécialisé est de $1,8 \%$ chez les garcons et de $1,2 \%$ chez les filles; au niveau 1, les $10 \%$ des élèves résidant dans les quartiers les plus défavorisés, le taux de fréquentation de l'enseignement spécialisé est par contre de $7,3 \%$ chez les garçons et de $4,5 \%$ chez les filles. 
Dans les niveaux primaire et secondaire, l'enseignement spécialisé accueille environ $4 \%$ des élèves scolarisés.

Les garçons constituent les deux tiers des effectifs de l'enseignement spécialisé.

On observe une augmentation significative de la part de l'enseignement spécialisé dans l'enseignement primaire, passant de $4,1 \%$ à $4,9 \%$ entre 1998-1999 et 2004-2005.

Entre 1993-1994 et 2005-2006, la répartition des élèves de l'enseignement spécialisé dans les différents types d'enseignement est demeurée très similaire, tant au niveau fondamental qu'au niveau secondaire.

a figure 1 présente l'évolution du nombre d'élèves dans l'enseignement spécialisé entre 1993-1994 et 2005-2006, selon le sexe et le niveau d'enseignement. Dans l'enseignement spécialisé, le nombre de garçons est supérieur à celui des filles : ils y forment deux tiers des effectifs alors qu'ils en représentent environ la moitié dans l'enseignement ordinaire.

Au cours de la période observée, le nombre d'élèves, filles et garçons, a augmenté tant au niveau fondamental (+21\%) qu'au niveau secondaire $(+14 \%)$, passant, dans le fondamental, de 8726 à 10436 garçons et de 4944 à 6149 filles et, dans le secondaire, de 8386 à 9509 garçons et de 4373 à 5089 filles. Entre 2004-2005 et 2005-2006, le nombre d'élèves de l'enseignement spécialisé est demeuré stable au niveau fondamental mais s'est encore accru de 2,7 \% dans le secondaire.

La figure 2 montre qu'à partir du primaire, l'enseignement spécialisé accueille environ $4 \%$ des élèves scolarisés. A titre de comparaison, la majorité des autres pays européens scolarise moins de $3 \%$ des enfants dans ce type de structure (Eurydice, 2005).

Au niveau primaire, la croissance des effectifs de l'enseignement spécialisé (figure 1) contraste, à partir de 1999-2000, avec la diminution des populations de l'enseignement ordinaire. Il en résulte une augmentation significative de la part de l'enseignement spécialisé dans l'enseignement primaire à partir de cette année, passant de 4,1 \% en 1998-1999 à 4,9\% en 2004-2005 (figure 2). Depuis 2001-2002, on assiste également à une légère augmentation de la part de l'enseignement spécialisé dans l'enseignement secondaire, passant de 3,7 \% en 2000-2001 à 3,9 \% en 2004-2005 et à $4 \%$ en 2005-2006, cette évolution demeure cependant trop récente et d'ampleur trop faible pour conclure à une réelle tendance à la hausse.

Les figures 3 et 4 présentent la répartition des élèves dans les différents types de l'enseignement spécialisé, respectivement pour le fondamental et le secondaire. Les deux figures comparent cette répartition entre l'année scolaire 1993-1994 et les deux dernières années scolaires disponibles, 2004-2005 et 2005-2006.

En 2005-2006, quatre des huit types d'enseignement accueillent près de neuf dixièmes de la population scolaire de l'enseignement fondamental spécialisé. Le type troubles instrumentaux scolarise $38,3 \%$ des élèves du fondamental spécialisé (38,8 \% en 2004-2005), le type arriération mentale légère en scolarise $24,5 \%$ (24,1 \% en 2004-2005), le type arriération mentale modérée ou sévère, 14,1 \% (14\% en 2004-2005), et le type troubles caractériels et/ou de personnalité, 10,7 \% (10,9\% en 2004-2005). En ce qui concerne les autres types d'enseignement, le type déficiences physiques accueille $5 \%$ des élèves du fondamental spécialisé (comme en 2004-2005), le type maladies en accueille 4,2 \% (4,1 \% en 2004-2005), le type déficiences auditives, 2,5\% (2,4\% en 2004-2005), et le type déficiences visuelles, 0,6 \% (comme en 2004-2005).

Entre 1993-1994 et 2005-2006, les parts relatives des huit types d'enseignement de l'enseignement fondamental spécialisé sont demeurées très stables.

L'enseignement secondaire spécialisé se répartit en sept types d'enseignement liés aux caractéristiques des élèves, le type troubles instrumentaux n'existant pas à ce niveau. La majorité des élèves $(53,7 \%$ en 2005-2006 contre $54 \%$ en 2004-2005) sont scolarisés dans le type arriération mentale légère. Deux autres types d'enseignement comptent également une part importante de la population scolaire : le type troubles caractériels et/ou de personnalité (18,7 \% en 2005-2006 contre $18,8 \%$ en 2004-2005) ainsi que le type arriération mentale modérée ou sévère (18,5 \% en $2005-2006$ contre $17,8 \%$ en $2004-2005)$. En ce qui concerne les autres types d'enseignement, le type déficiences physiques scolarise $5,3 \%$ des élèves du secondaire spécialisé en 2005-2006 (5,5 \% en 20042005), les types maladies et déficiences auditives en accueillent chacun environ 1,5\%, tandis que le type déficiences visuelles en scolarise 0,9\%.

De 1993-1994 à 2005-2006, comme au niveau fondamental, la répartition relative des élèves entre les différents types d'enseignement est demeurée très stable dans l'enseignement secondaire spécialisé. 


\subsection{Nombre d'élèves selon le sexe dans l'enseignement fondamental et secondaire spécialisé - Années scolaires 1993-1994 à 2005-2006}

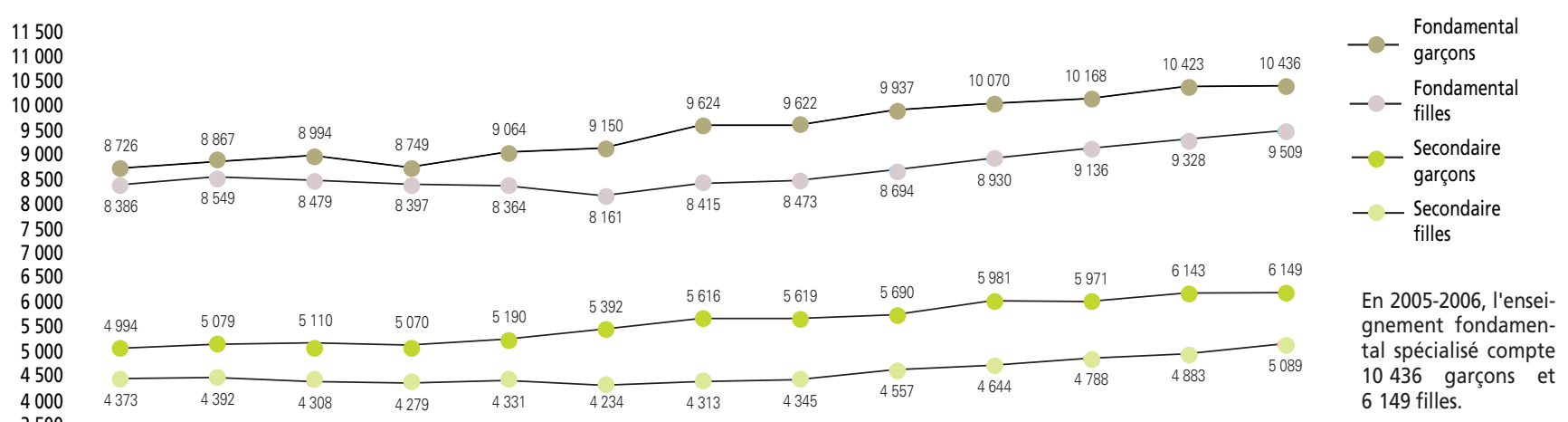

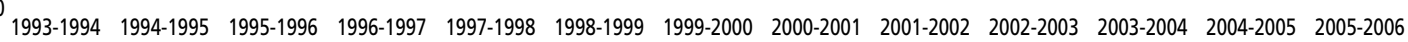

\subsection{Part de l'enseignement spécialisé dans l'enseignement fondamental et secondaire de plein exercice - Années scolaires 1993-1994 à 2005-2006}

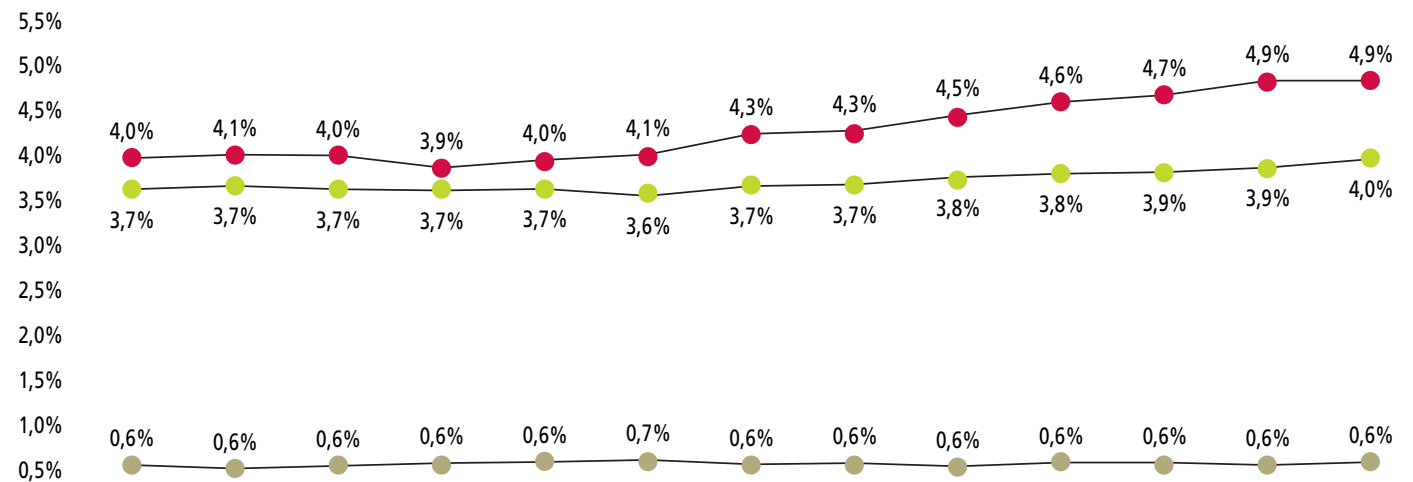

0,0\% 1993-1994 1994-1995 1995-1996 1996-1997 1997-1998 1998-1999 1999-2000 2000-2001 2001-2002 2002-2003 2003-2004 2004-2005 2005-2006

10.3 Répartition des élèves dans les différents types de l'enseignement fondamental spécialisé - Comparaison 1993-1994, 2004-2005 et 2005-2006

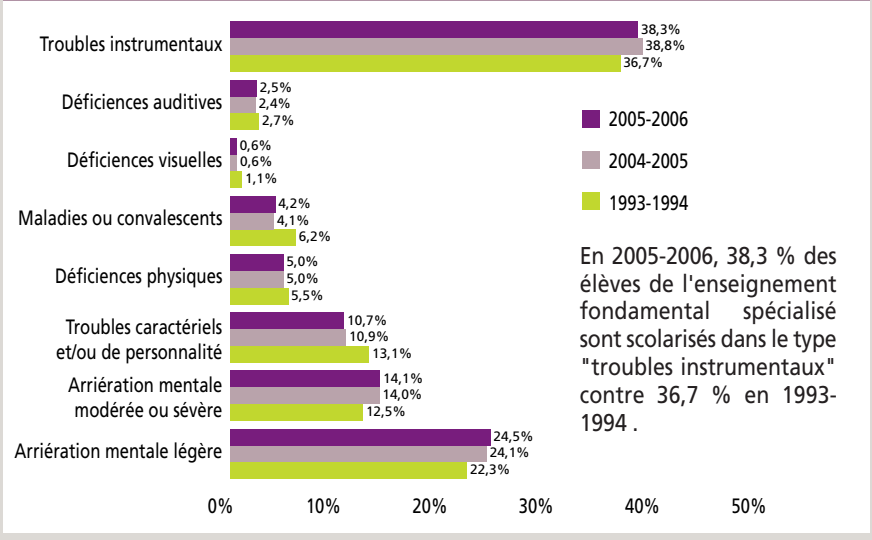

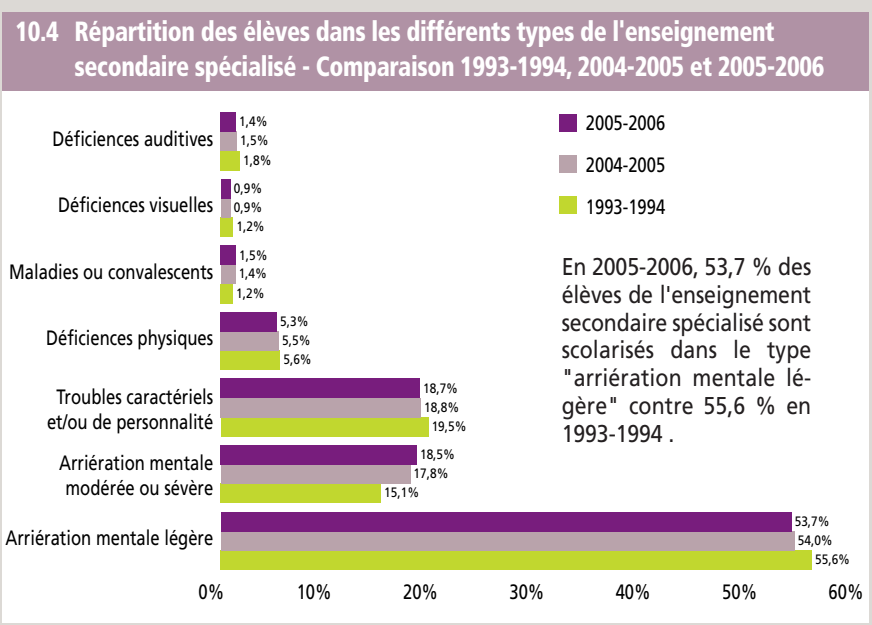

(c) Ministère de la Communauté française de Belgique/ETNIC - 200725
Maternel - part du spécialisé

Primaire - part du spécialisé

Secondaire (de plein exercice) - part du spécialisé

En 2005-2006, 4,9 \% des élèves de l'enseignement primaire sont scolarisés dans l'enseignement spécialisé contre $4 \%$ en 1993-1994. 


\begin{abstract}
La part des élèves de nationalité étrangère dans l'enseignement spécialisé est plus élevée que dans l'enseignement ordinaire.
Deux facteurs se conjuguent pour expliquer cette situation : d'une part, l'attrait extérieur exercé sur les élèves de nationalité française par notre structure d'enseignement spécialisé et, d'autre part, l'origine socioculturelle et socio-économique déterminant une proportion plus importante de certaines populations dans la fréquentation de l'enseignement spécialisé.
\end{abstract}

'importance relative des élèves de nationalité étrangère dans l'enseignement spécialisé est présentée sous trois facettes. La première approche (figure 1) relève la part des élèves de nationalité étrangère dans l'enseignement spécialisé en comparaison avec l'enseignement ordinaire. La deuxième approche (figures 2 et 3 ) présente les parts relatives des principales nationalités étrangères. Enfin, la troisième approche (figures 4 et 5) montre la part des élèves dans l'enseignement spécialisé selon leur nationalité.

Tant au niveau fondamental qu'au niveau secondaire, l'enseignement spécialisé compte une part plus importante d'élèves de nationalité étrangère (figure 1). En 2005-2006, on en compte 15,6\% dans l'enseignement maternel spécialisé contre 9,5\% dans l'enseignement maternel ordinaire, $12,8 \%$ dans l'enseignement primaire spécialisé contre 9,1\% dans l'enseignement primaire ordinaire et 20,7 \% dans l'enseignement secondaire spécialisé contre $10,5 \%$ dans l'enseignement secondaire ordinaire de plein exercice.

L'évolution à la baisse de la part des élèves de nationalité étrangère entre 1993-1994 et 2005-2006 apparaît par contre similaire dans les deux formes d'enseignement. L'augmentation du nombre d'acquisitions de la nationalité belge au cours de la période en est très vraisemblablement la principale explication. Dans le maternel, la part des élèves de nationalité étrangère se réduit de l'ordre de $5 \%$, passant de $14,5 \%$ à $9,5 \%$ dans l'ordinaire et de $20,5 \%$ à $15,6 \%$ dans le spécialisé ; dans le primaire, cette part se réduit de l'ordre de $8 \%$, passant de $16,8 \%$ à $9,1 \%$ dans l'ordinaire et de $22,2 \%$ à $12,8 \%$ dans le spécialisé ; dans le secondaire, cette même part se réduit de quelque $7 \%$, passant de $17,9 \%$ à $10,5 \%$ dans l'ordinaire de plein exercice et de $27,4 \%$ à $20,7 \%$ dans le spécialisé.

La deuxième facette (figures 2 et 3 ) montre la part importante de Français dans l'enseignement spécialisé (il correspond au double de leur part dans l'enseignement ordinaire), tant au niveau fondamental qu'au niveau secondaire, sur la période observée (de 1993-1994 à 2005-2006). Ainsi, en 2005-2006, la part des Français par rapport à l'ensemble des élèves de nationalité étrangère dans l'enseignement secondaire spécialisé s'élève à $68 \%$, contre $32 \%$ dans l'enseignement secondaire ordinaire de plein exercice ; ces deux mêmes parts, dans l'enseignement primaire, sont respectivement de $41 \%$ et $19 \%$, et, dans l'enseignement maternel, de $37 \%$ et $19 \%$. Un tel écart entre les deux formes d'enseignement s'explique probablement par un attrait extérieur, exercé sur la France en l'occurrence, de notre structure d'enseignement spécialisé.

La troisième facette (figures 4 et 5) indique qu'en 2005-2006, la proportion ${ }^{1}$ des élèves de nationalité belge fréquentant l'enseignement spécialisé est de 3,1 \% dans le fondamental et de 3,5 \% dans le secondaire. Pour les principales autres nationalités fréquentant l'enseignement en Communauté française, cette proportion est supérieure : respectivement de 4,6 et $5 \%$ pour les élèves de nationalité marocaine, de 4,3 et $4,9 \%$ pour les élèves de nationalité turque et de 5,7 et $4,6 \%$ pour les élèves de nationalité congolaise. L'origine socioculturelle et socio-économique de ces populations peut ici être évoquée pour expliquer cette part supérieure dans la fréquentation de l'enseignement spécialisé. La proportion des élèves de nationalité française dans l'enseignement spécialisé est quant à elle encore nettement plus élevée, puisqu'elle est de 9,4\% dans l'enseignement fondamental et de $14,6 \%$ dans l'enseignement secondaire. Le facteur attractif de notre structure d'enseignement spécialisé évoqué précédemment est, dans ce cas, l'explication de cette concentration.

Il apparaît également que la part relative de fréquentation de l'enseignement spécialisé a augmenté entre 1993-1994 et 2005-2006, mais dans des proportions différentes selon la nationalité : de $0,1 \%$ pour les élèves de nationalité marocaine, de $0,4 \%$ pour les élèves de nationalité belge, de $0,9 \%$ pour les élèves turcs, de $2,5 \%$ pour les élèves de nationalité congolaise et de $3 \%$ pour les élèves français. 


\subsection{Part des élèves de nationalité étrangère dans l'enseignement spécialisé}

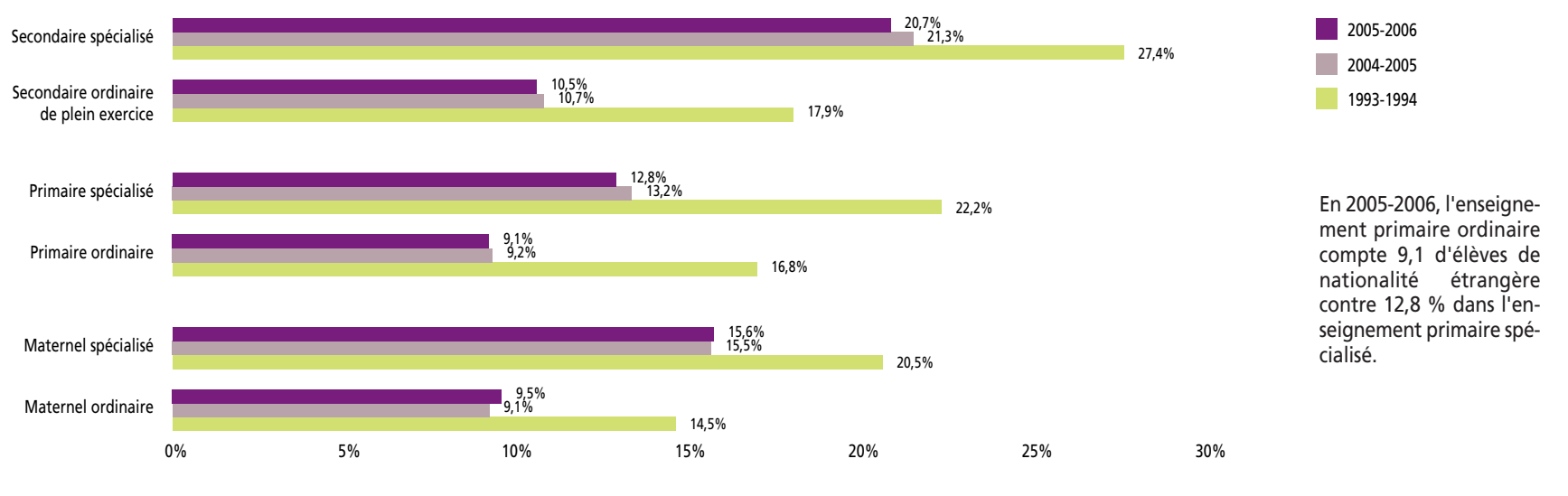

11.2 Répartition des élèves de nationalité étrangère dans l'enseignement spécialisé et ordinaire en 1993-1994

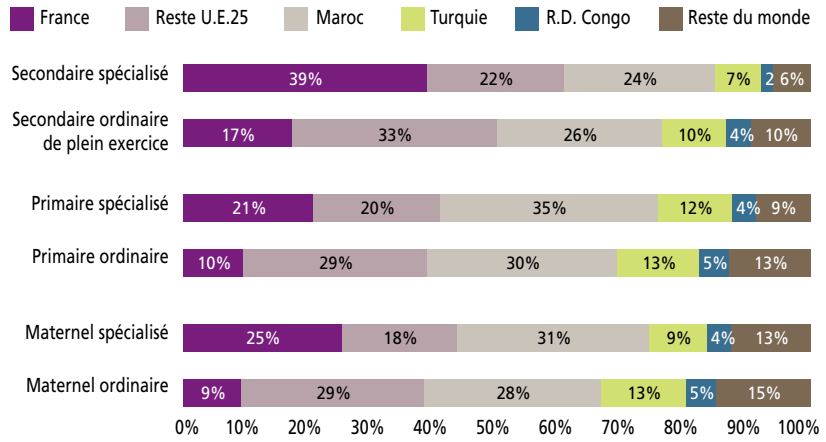

En 1993-1994, 39 \% des élèves de nationalité étrangère sont de nationalité française dans l'enseignement secondaire spécialisé.

\subsection{Proportion des élèves de l'enseignement fondamental fréquentant l'enseignement spécialisé, selon la nationalité 1993-1994, 2004-2005 et 2005-2006}

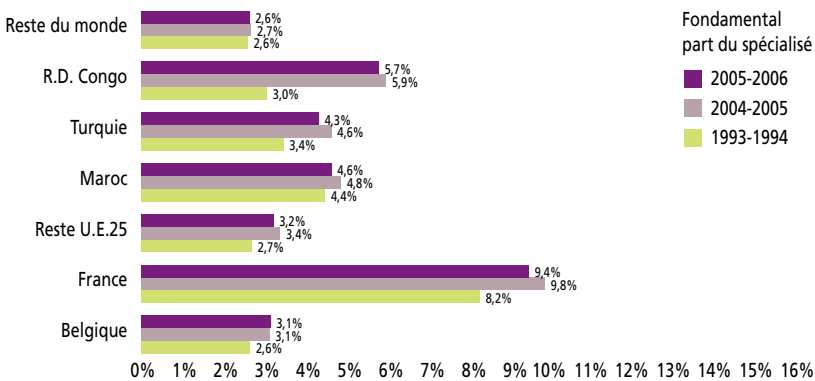

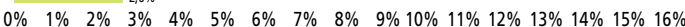

En 2005-2006, 9,4\% des élèves de nationalité française de l'enseignement fondamental sont scolarisés dans l'enseignement spécialisé contre $3,1 \%$ des élèves belges.
11.3 Répartition des élèves de nationalité étrangère dans l'enseignement spécialisé et ordinaire en 2005-2006

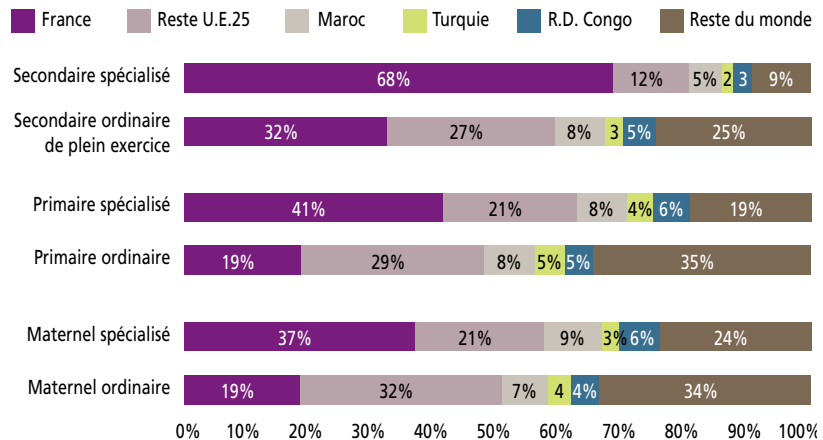

En 2005-2006, 68 \% des élèves de nationalité étrangère sont de nationalité française dans l'enseignement secondaire spécialisé.

11.5 Proportion des élèves de l'enseignement secondaire fréquentant l'enseignement spécialisé, selon la nationalité 1993-1994, 2004-2005 et 2005-2006

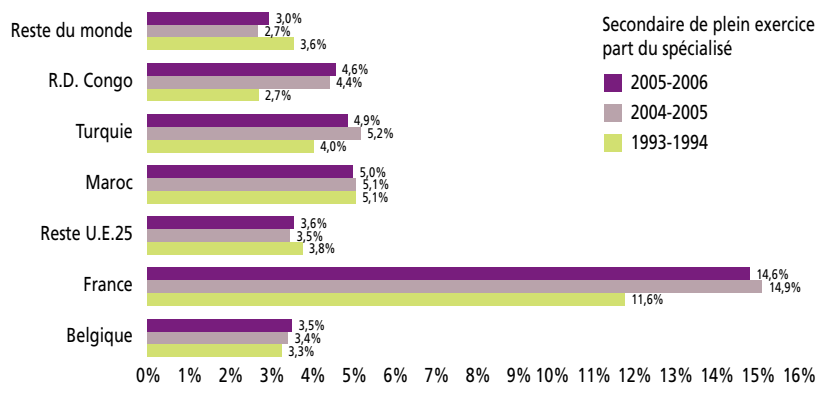

En 2005-2006, 14,6\% des élèves français de l'enseignement secondaire de plein exercice sont scolarisés dans l'enseignement spécialisé contre 3,5\% des élèves belges. 


\begin{abstract}
L'enseignement général en Communauté française attire, en 2005-2006, 47 \% de la population de l'enseignement secondaire ordinaire de plein exercice, pourcentage très stable dans le temps.

Parmi les élèves de l'enseignement secondaire au $2^{\mathrm{e}}$ degré, deux sur cinq se trouvent dans la forme qualifiante alors qu'au $3^{\mathrm{e}}$ degré, ils sont près d'un sur deux.

A 15 ans, plus d'un élève sur deux est à l'heure alors qu'à 17 ans, il n'y en a plus que deux sur cinq. La dispersion des élèves tout au long du parcours dans l'enseignement secondaire ordinaire de plein exercice augmente avec l'âge et pourrait expliquer les résultats obtenus lors des évaluations PISA mais aussi les abandons précoces en fin de parcours.
\end{abstract}

et indicateur dresse un portrait du public de l'enseignement secondaire ordinaire de plein exercice, particulièrement celui des $2^{\mathrm{e}}$ et $3^{\mathrm{e}}$ degrés. Il décrit les taux de fréquentation des différentes sections et formes d'enseignement, ainsi que les répartitions des effectifs à des âges donnés.

La figure 1 présente l'évolution des effectifs fréquentant les formes générale, technique de transition, technique de qualification et professionnelle, par sexe, de 1990-1991 à 2005-2006. On y observe la part prépondérante de la forme générale regroupant près de la moitié des effectifs. Elle se caractérise également par une présence majoritaire de filles, contrairement aux autres formes. En outre, c'est principalement dans l'enseignement qualifiant que l'on observe une évolution significative des effectifs : depuis 8 ans, la forme technique de qualification prend le dessus sur la forme professionnelle, particulièrement chez les filles.

La figure 2 représente l'évolution de la répartition des effectifs par forme et par section, sur 15 ans, pour le $2^{\mathrm{e}}$ et pour le $3^{\mathrm{e}}$ degrés. Ces deux degrés présentent des répartitions d'élèves différentes selon la section et la forme suivies : en 2005-2006, 2 élèves sur 5 choisissent la section de qualification au $2^{\mathrm{e}}$ degré (appelé aussi degré d'orientation); il y en a 1 sur 2 au $3^{\text {e }}$ degré (appelé degré de détermination). Depuis 1990-1991, on observe dans le $2^{\mathrm{e}}$ degré une augmentation importante du choix de la forme technique de qualification qui se confirme dans le $3^{\text {e }}$ degré. Par contre, la forme générale prend, depuis 15 ans, une part plus importante de l'effectif dans le $2^{\mathrm{e}}$ degré et une moindre part dans le $3^{\mathrm{e}}$ degré. Ces deux graphiques mettent en évidence les transferts d'effectifs entre les formes durant le parcours scolaire à mettre en lien avec les attestations d'orientation délivrées ${ }^{1}$ de la $3^{\mathrm{e}}$ à la $5^{\mathrm{e}}$.

Afin de prendre en compte les rythmes scolaires individuels des élèves aux $2^{\mathrm{e}}$ et $3^{\mathrm{e}}$ degrés de l'enseignement secondaire de plein exercice, la figure 3 présente la répartition des élèves ayant 15 ans en 2003-2004 et des élèves ayant 17 ans en 2005-2006 et fréquentant cet enseignement. On remarque une déperdition de 3915 élèves de cette cohorte (soit 7,5\% de l'effectif des 15 ans). Les filles y sont systématiquement plus « à l'heure " que les garçons.

À 15 ans, plus de la moitié des élèves (52,3\%) sont « à l'heure " (en $4^{\mathrm{e}}$ année) et $1,5 \%$ (798 élèves) fréquentent déjà le $3^{\mathrm{e}}$ degré. Par ailleurs on remarque que la part cumulée des élèves en $3 \mathrm{P}$ et $3 \mathrm{TQ}$ en $3^{\mathrm{e}}$ année est plus importante que la part cumulée des élèves en 4P et 4TQ « à l'heure » en $4^{\mathrm{e}}$ année, ceci à l'inverse de la section de transition. Sachant que les évaluations internationales "PISA » examinent les compétences des élèves de 15 ans, cette dispersion des effectifs dans le parcours du secondaire ordinaire de plein exercice, liée à une grande variation du rythme scolaire, peut expliquer une part des résultats obtenus ${ }^{2}$.

À 17 ans, il n'y a plus que $41,3 \%$ des élèves présents dans l'enseignement ordinaire de plein exercice qui sont à l'heure (en $6^{\mathrm{e}}$ année). La dispersion des élèves de 17 ans est importante : ainsi on retrouve, dans la section qualifiante, $10 \%$ des effectifs en $6^{\mathrm{e}}, 21 \%$ en $5^{\mathrm{e}}$ et encore $13 \%$ en $4^{\mathrm{e}}$. Sachant que ces élèves atteignent l'âge de 18 ans à la fin de l'année d'études en cours, on peut faire l'hypothèse d'un taux important de départs durant le $2^{\mathrm{e}}$ degré vers d'autres types d'enseignement comme l'alternance, l'apprentissage, ou encore d'abandons scolaires, sans obtention de certification ${ }^{3}$.

1 Voir l'indicateur 16 - Attestations d'orientation délivrées par les Conseils de classe dans l'enseignement secondaire ordinaire de plein exercice.

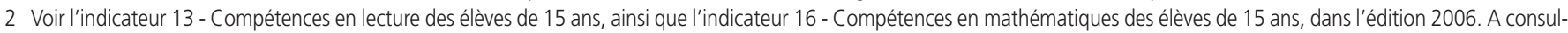
ter aussi sur le site : www.enseignement.be

3 Voir l'indicateur 26 - Taux de certification en 6 e année de l'enseignement secondaire de plein exercice. 
12.1 Évolution des effectifs dans les $2^{\mathrm{e}}$ et $3^{\mathrm{e}}$ degrés du secondaire ordinaire de plein exercice, selon la forme fréquentée, par sexe, de 1990-1991 à 2005-2006

Garçons

Filles

$30 \%$

$30 \%$

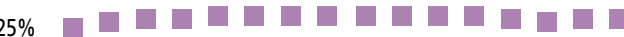

a $\mathrm{G}$

$25 \%$

$20 \%$

$\square \square \square \square \square \square \square \square \square \square \square \square \square \square \square \square$

$20 \%$

TT

$15 \%$

$10 \%$

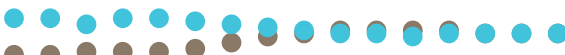

$15 \%$

$10 \%$

$5 \% \quad \square \square \square \square \square \square \square \square \square \square \square \square \square \square \square \square$

$5 \% \quad \square \square \square \square \square \square \square \square \square \square \square \square \square \square \square \square$

$0 \%$ $0 \%$

$90-91 \quad 93-94 \quad 96-97 \quad 99-00 \quad 02-03 \quad 05-06$

96-97

$99-00$

$02-03$

05-06

- TQ

- $\mathrm{P}$

12.2 Évolution de la répartition des élèves inscrits dans les $2^{\mathrm{e}}$ et $3^{\mathrm{e}}$ degrés de l'enseignement ordinaire de plein exercice selon la forme et la section fréquentée, de 1990-1991 à 2005-2006

$2^{\mathrm{e}}$ degré

$60 \%$

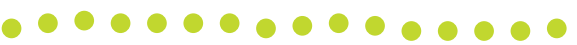

$50 \%$

$40 \%$

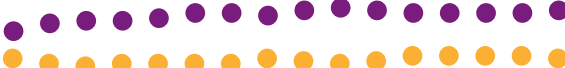

$30 \%$

$20 \%$

$10 \%$

$0 \%$

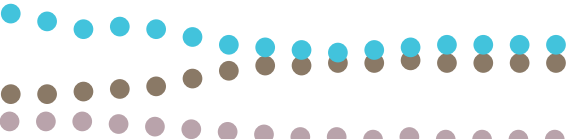

$\begin{array}{llllll}90-91 & 93-94 & 96-97 & 99-00 & 02-03 & 05-06\end{array}$ $3^{e}$ degré

G

$60 \%$

\% $40 \%$

$30 \%$

$10 \%$

$0 \%$

90-91
En 2005-2006, $11 \%$ de l'effectif sont des filles fréquentant l'enseignement technique de qualification dans les $2^{\mathrm{e}}$ et $3^{\mathrm{e}}$ degrés de l'enseignement secondaire ordinaire de plein exercice.

12.3 Répartition des élèves de 15 ans (en 2003-2004) et de 17 ans (en 2005-2006) inscrits dans l'enseignement secondaire ordinaire de plein exercice par degré ou année d'études et par sexe

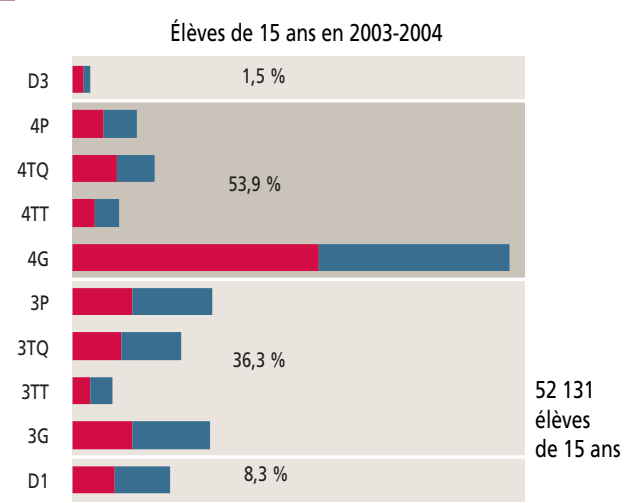

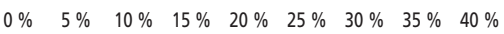

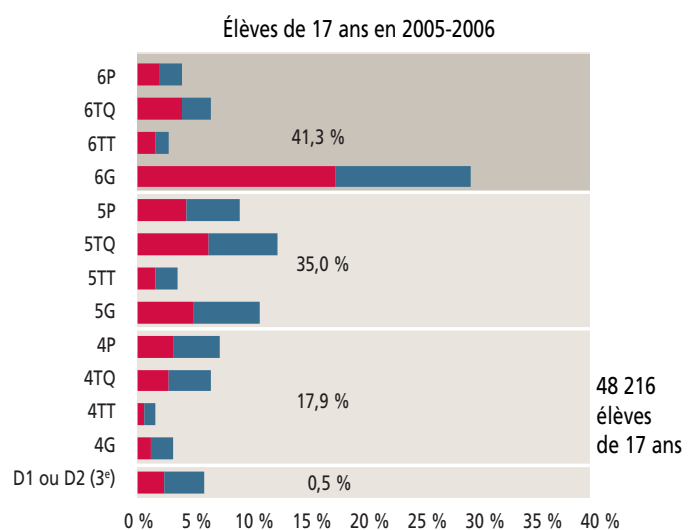

- Garçons

a Filles

En 2003-2004, il y a 52131 élèves de 15 ans qui fréquentent l'enseignement secondaire ordinaire de plein exercice dont $53,9 \%$ sont " à I'heure " en 4 alors qu'il n'y a plus que $41,3 \%$ des 48216 élèves de 17 ans fréquentant ce même enseignement en 2005-2006 qui sont " à I'heure» en 6 e 
En Communauté française, les élèves réalisent « normalement « un parcours de douze ans dans l'enseignement obligatoire. Une proportion importante d'élèves s'écarte de cette norme.

En 2005-2006, en moyenne, près d'un élève sur 5 est en retard scolaire en primaire et c'est le cas de près d'un élève sur 2 en secondaire. Ces proportions ont évolué dans le temps, en relation, entre autres avec certaines modifications des structures de l'enseignement. Des différences s'observent également selon le sexe et les formes d'enseignement choisies.

$E_{\text {entitan }}^{n}$ Communauté française, un enfant ayant 6 ans durant l'année civile entre en première année primaire. En fin de secondaire, après un parcours de douze ans, il doit donc sortir l'année de ses 18 ans. Cela n'est pas souvent le cas : sur l'ensemble du parcours, on observe un pourcentage élevé d'élèves présentant un retard scolaire ${ }^{1}$. Afin de visualiser ce phénomène, on peut représenter les rythmes scolaires individuels ${ }^{2}$ en 20052006 (figures 1 et 4) mais, pour mieux comprendre les origines possibles du retard, il est nécessaire d'en étudier l'évolution dans le temps (figures 2 et 3 ).

La figure 1 montre en 2005-2006, une progression du retard scolaire quasi linéaire (en lien avec l'accumulation du retard au fil des années) dès la $3^{\mathrm{e}}$ maternelle ${ }^{3}$ : en $5^{\mathrm{e}}$ primaire, plus d'un élève sur 5 est en retard; en $4^{\mathrm{e}}$ secondaire, c'est le cas pour plus d'un élève sur 2 . II y a néanmoins deux sauts importants : d'une part entre la $6^{\mathrm{e}}$ primaire et la $1^{\text {re }}$ secondaire (de $22 \%$ à $34 \%$ de retard), puis d'autre part entre les $2^{\mathrm{e}}$ et $3^{\mathrm{e}}$ années de l'enseignement secondaire (le retard passe de $35 \%$ à $47 \%$ ). Dans l'enseignement primaire, on peut réaliser le parcours en 6 ans, voire 7 et exceptionnellement en 8 ans $^{4}$ et, de ce fait, un certain nombre d'élèves quittent prématurément la $5^{\mathrm{e}}$ ou même la $4^{\mathrm{e}}$ primaire pour entrer en $1^{\text {re }}$ année secondaire, ce qui peut expliquer le taux de retard moins important en $6^{\mathrm{e}}$ primaire. De même, la diminution du retard scolaire en $6^{\mathrm{e}}$ secondaire peut s'expliquer par les départs vers l'enseignement ou la formation en alternance et les abandons, principalement pour des élèves ayant atteint l'âge de 18 ans, limite de l'obligation scolaire.

La figure 2 donne une approche globale des taux de retard scolaire durant les 17 dernières années. En moyenne, un élève sur 5 est en retard scolaire en primaire et près de 1 sur 2 en secondaire. On observe une diminution lente et continue du taux de retard jusqu'en 1998-1999. À partir de l'année suivante, cette tendance s'inverse tant dans le primaire que dans le secondaire. Invariablement, les garçons sont plus en retard que les filles et cette différence augmente durant le parcours scolaire.

Pour analyser plus finement les moments de production du retard scolaire, la figure 3 décrit les taux de retard en début et fin de primaire (en $5^{\mathrm{e}}$, vu l'anomalie notée en $6^{\mathrm{e}}$ ) ainsi que, dans l'enseignement secondaire, au $1^{\mathrm{er}}$ degré, qui a connu plusieurs réformes, et au début des $2^{\mathrm{e}}$ et $3^{\mathrm{e}}$ degrés, moments d'orientation scolaire. En moyenne, en $1^{\text {re }}$ année primaire, plus d' 1 élève sur 10 est en retard scolaire et plus d' 1 sur 5 en $5^{\mathrm{e}}$ année primaire. En $1^{\mathrm{re}}$ secondaire, on passe de $39,3 \%$ de retard en 1989-1990 à un minimum de $25,3 \%$ en $2000-2001$. En $2^{\mathrm{e}}$, cette variation se répercute sur les taux observés : $50,1 \%$ en 1989-1990 à un minimum de 30,9 \% en 1999-2000. Au début du secondaire, la tendance à la diminution semble s'inverser. À partir de 1995-1996, la diminution nette du retard scolaire en début de secondaire (et ses effets sur le reste du parcours) peut s'expliquer comme une conséquence de la réforme instaurée dans le premier degré 5 , lequel doit dorénavant s'effectuer en un maximum de trois ans. Le faible impact de cette réforme sur la fin du secondaire suppose une production simultanée et plus importante de retard scolaire en $4^{\mathrm{e}}, 5^{\mathrm{e}}$ et $6^{\mathrm{e}}$ secondaires.

Pour analyser les lieux de production du retard scolaire, la figure 4 présente l'état du retard des élèves en $6^{\mathrm{e}}$ primaire et au début des $2^{\mathrm{e}}$ et $3^{\mathrm{e}}$ degrés en 2005-2006 selon la forme suivie. Les différences dans la structure des retards en fonction des formes choisies sont importantes. Le retard moyen d'un élève en $3^{\mathrm{e}}$ est d'environ 4 mois dans le général ; il passe à 8 mois dans le technique de transition, à plus d'un an dans le technique de qualification et à 1 an et 3 mois dans le professionnel. Les filles sont systématiquement plus à l'heure que les garçons, avec toutefois un écart moins important dans la section qualifiante. Sur base de l'étude des retards scolaires, un phénomène de relégation apparaît à l'entrée du deuxième degré, moment de l'orientation, et se renforce à l'abord du troisième degré, moment de confirmation de la section et de la forme choisies.

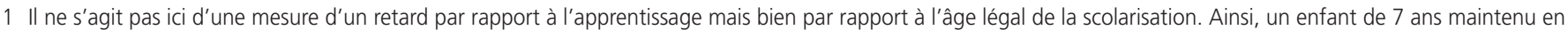
maternelle à 6 ans et entrant en $1^{\text {re }}$ primaire à 7 ans est considéré en retard durant toute sa scolarité même si celle-ci se passe sans redoublement.

2 Un élève est dit « à l'heure » s'il a au plus l'âge légal de scolarisation dans l'année d'études où il se trouve, sinon il sera dit " en retard scolaire».

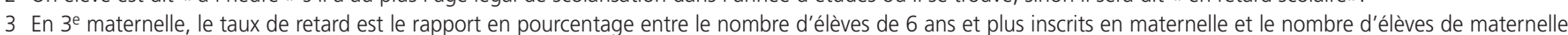
âgés de 5 ans et plus.

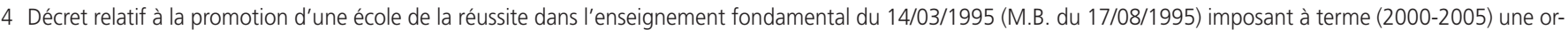
ganisation en cycles à l'intérieur desquels l'élève parcourt sa scolarité de manière continue, à son rythme et sans redoublement.

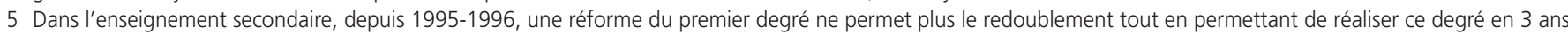
maximum. On a donc instauré une année complémentaire en fin de $2^{\mathrm{e}}$. Depuis 2000-2001, il est également possible d'effectuer en fin de 1 re cette année complémentaire. 


\subsection{Rythmes scolaires individuels dans l'enseignement ordinaire - Année scolaire 2005-2006}

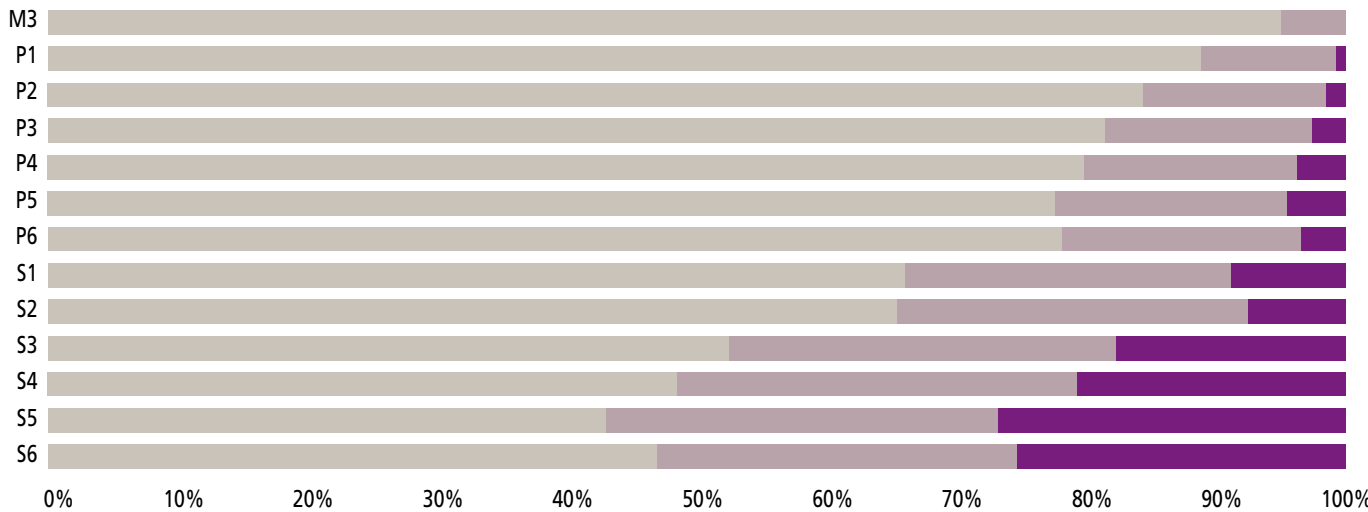

\section{Al'heure \\ Retard d'un an \\ Retard de 2 ans et plus}

En $4^{e}$ année de l'enseignement primaire ordinaire (P4), $80 \%$ des élèves sont à l'heure, $16 \%$ en retard scolaire d'un an, $4 \%$ en retard de 2 ans et plus; en 4 année du secondaire ordinaire (S4), $48 \%$ des élèves sont à l'heure, $31 \%$ sont en retard scolaire d'un an, $21 \%$ de 2 ans ou plus.
13.2 Évolution du taux de retard scolaire par sexe dans l'enseignement ordinaire - Années 1989-1990 à 2005-2006

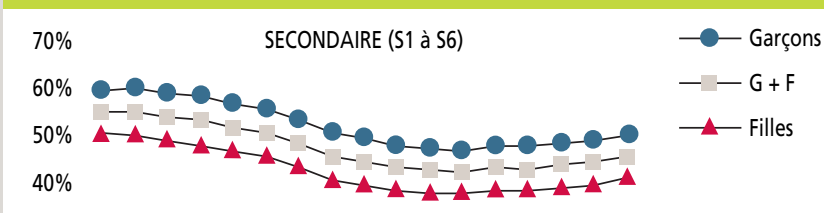

$30 \% \quad$ PRIMAIRES (P1 à P6)

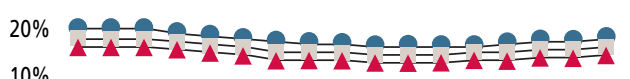

$0 \%$
$89-90$
93-94
$97-98$
01-02
$05-06$

En 2005-2006, dans le primaire, $20 \%$ des garçons et $16 \%$ des filles sont en retard scolaire d'au moins un an ; dans le secondaire, ce sont $51 \%$ des garçons et $41 \%$ des filles qui sont en retard scolaire d'au moins un an.

\section{3 Évolution du taux de retard scolaire dans certaines années d'études de} lenseignement ordinaire - Années 1989-1990 à 2005-2006

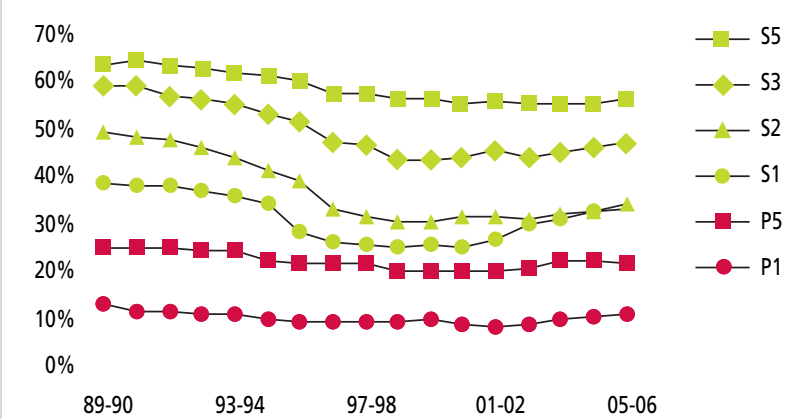

En 2005-2006, $11 \%$ des élèves de $1^{\text {re }}$ année primaire ( $\mathrm{P} 1$ ) sont en retard et $22 \%$ le sont en $5^{\mathrm{e}}$ primaire (P5); dans le secondaire ordinaire (toutes sections confondues), le taux de retard s'élève à $35 \%$ en $2^{\mathrm{e}}$ année (S2) et à $57 \%$ en $5^{\mathrm{e}}$ année (S5).

\subsection{Rythmes scolaires individuels dans l'enseignement ordinaire selon la forme et le sexe en $6^{\circ}$ année primaire, $3^{\circ}$ et $5^{\circ}$ années secondaires - Année scolaire $2005-2006$}

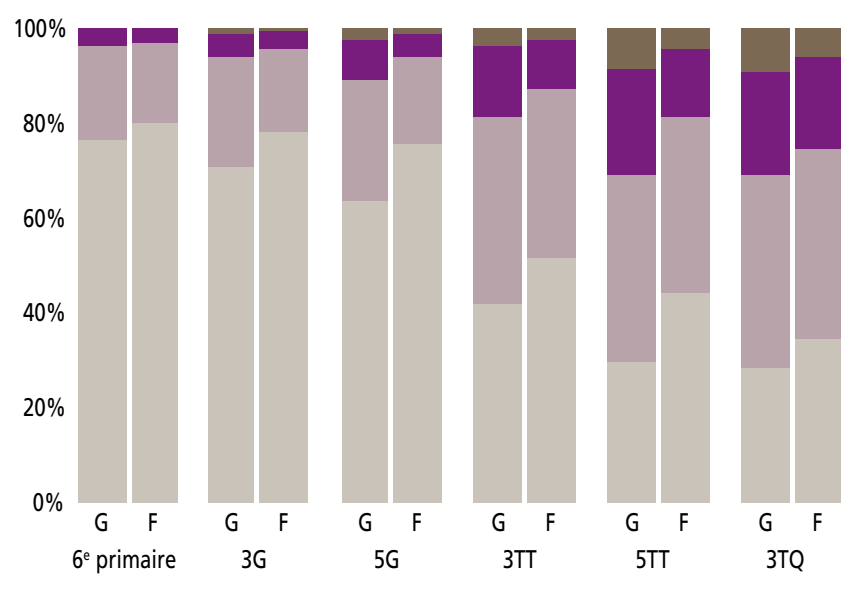

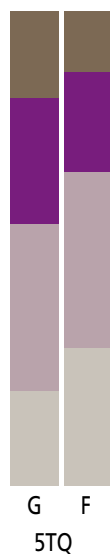

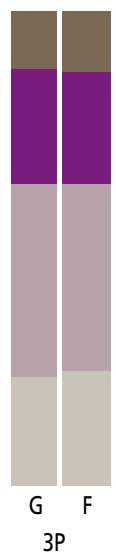

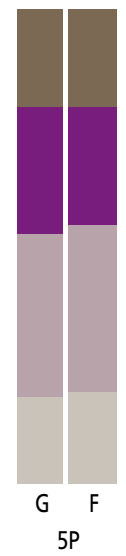

Retard de 3 ans et +

Retard de 2 ans

Retard d'un an

A l'heure

En 2005-2006, les filles présentes en $6^{\text {e }}$ primaire sont près de $80 \%$ « à l'heure "; ce taux est encore de $78 \%$ dans les classes de $3^{\mathrm{e}}$ secondaire générale (3G); en $3^{e}$ technique de transition (y compris la forme artistique de transition) (3TT), elles ne sont plus que $52 \%$; en $3^{e}$ technique de qualification (y compris la forme artistique de qualification) (3TQ) environ $34 \%$ et en $3^{\mathrm{e}}$ professionnelle (3P) $24 \%$. En $5^{5}$ générale $(5 \mathrm{G}), 63 \%$ des garçons sont à l'heure pour $76 \%$ de filles. 


\section{Redoublement dans l'enseignement ordinaire de plein exercice}

L'étude longitudinale des taux de redoublement montre que dans une classe de l'école primaire, en moyenne, plus d'1 élève sur 25 redouble, alors qu'à l'école secondaire, on dénombre plus de 3 élèves redoublants sur 25.

Le taux de redoublement subit des variations parfois importantes au cours du temps mais aussi selon les années et les formes d'études considérées.

es redoublements ${ }^{1}$ dans l'enseignement ordinaire de plein exercice ${ }^{2}$

sont traités ici par une observation de leur évolution de 1992-1193 à 2005-2006, dans l'enseignement primaire d'abord, dans le secondaire ensuite.

Dans l'enseignement primaire, la figure 1 montre que le taux de redoublement est le plus élevé en $1^{\text {re }}$ et $2^{\mathrm{e}}$ années (de l'ordre de $6 \%$ ). Cette caractéristique du début du parcours scolaire obligatoire est stable dans le temps. Cela dit, en $1^{\text {re }}$ année, on observe une diminution régulière du taux de redoublement jusqu'en 1999-2000, puis une chute plus forte en 2000-2001 suivie d'une remontée. En $2^{\mathrm{e}}$ année, le taux de redoublement, relativement stable jusqu'en 1999-2000, remonte ensuite. Le décret ${ }^{3}$ permettant depuis 2000 le redoublement en fin de $2^{\mathrm{e}}$ année uniquement pourrait expliquer la chute du taux de redoublement en $1^{\text {re }}$ et l'accroissement de ce taux en $2^{\mathrm{e}}$. De la $3^{\mathrm{e}}$ à la $6^{\mathrm{e}}$ primaire, les taux de redoublement varient autour de $3 \%$. Ils changent peu durant la période analysée même si on observe une tendance à la diminution qui s'inverse à partir de 2001-2002. La 6 e présente les taux de redoublement les plus bas. Une explication en serait que, sauf dérogation, les élèves de 13 ans ou ayant déjà redoublé en primaire passent directement en secondaire.

La figure 2 affiche, par année d'études, les taux de redoublement dans l'enseignement secondaire de 1992-1993 à 2005-2006. Le $1^{\mathrm{er}}$ degré présente des caractéristiques particulières. En $1^{\text {re }}$ année, le taux de redoublement s'articule autour de deux années charnières : en 1995-1996, il chute de 8 à $2 \%$ alors qu'en 2002-2003, après une période de stabilité, il atteint à nouveau les $8 \%$. Depuis, il a encore progressé pour atteindre $10,7 \%$ en 2005-2006. En $2^{\mathrm{e}}$ année, le taux de redoublement diminue jusqu'en 1996-1997 où il est de 6,2 \%, puis augmente régulièrement pour atteindre $10,4 \%$ en 2002-2003, diminue à nouveau en 2003-2004 (7,4 \%) et augmente en 2004-2005 et en 2005-2006 (7,7\% et 7,8\%). Cette dynamique des taux de redoublement est à mettre en lien avec la réforme du $1^{\text {er }}$ degré. Depuis $1995^{4}$, ce degré doit en effet être réalisé en 3 ans maximum. Une " $2 \mathrm{CC}$ ", année complémentaire après la $2^{\mathrm{e}}$, a donc été instaurée. Depuis $2001^{5}$, il est également possible d'effectuer une " $1 \mathrm{AC}$ ", année complémentaire en fin de $1^{\text {re }}$, pourvu que le $1^{\text {er }}$ degré soit effectué en 3 ans seulement ${ }^{6}$. Les $3^{\mathrm{e}}, 4^{\mathrm{e}}$ et $5^{\mathrm{e}}$ années du secondaire présentent des taux de redoublement aux profils proches, avec, en $3^{\mathrm{e}}$ et $5^{\mathrm{e}}$, les taux les plus élevés. Ces taux augmentent régulièrement au fil du temps (jusqu'à 19,2\% pour la $3^{\mathrm{e}}$ en 2005-2006) avec cependant une inversion de la tendance en $5^{\mathrm{e}}$ ces dernières années. Les taux de redoublement plus bas en $6^{\mathrm{e}}(7,1 \%$ en $2005-2006)$ peuvent entre autres être liés aux abandons en fin de parcours (décrochage scolaire, arrivée à l'âge de la majorité et donc fin de l'obligation scolaire, choix de l'enseignement en alternance ou de promotion sociale).

Les taux de redoublement observés en $3^{\mathrm{e}}$ et $5^{\mathrm{e}}$ années de l'enseignement secondaire méritent une analyse plus fine, selon les sections et formes d'enseignement, car ce sont deux moments clés dans l'orientation en secondaire. Dans la figure 3, on observe que, pour les formes techniques, le taux de redoublement a progressé régulièrement particulièrement en $3^{e}$ année : en 2005-2006, en moyenne, plus d' 1 élève sur 4 est redoublant en $3^{\mathrm{e}}$ année du technique de transition et 1 sur 3 en $3^{\mathrm{e}}$ année du technique de qualification. La pertinence des choix d'orientation est peut-être en cause. Pour l'enseignement professionnel, le taux de redoublement progresse également mais moins fortement que dans les formes techniques (en 2005-2006, 23,2\% en $3^{\mathrm{e}}$ et $19,9 \%$ en $5^{\mathrm{e}}$ ). Cette moindre progression est peut-être en relation avec le taux d'abandon en fin de parcours scolaire.

Notons enfin la chute significative des taux de redoublement en 1996-1997, qui résulte vraisemblablement des grèves qui se sont déroulées durant le premier semestre 1996.

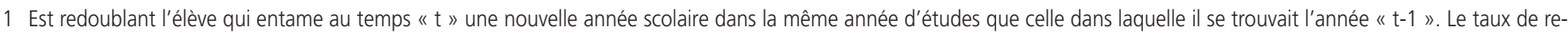
doublement est alors calculé par rapport à l'effectif de cette année d'études au temps « $t$ ».

2 Ne sont pas pris en compte ici les élèves fréquentant l'enseignement en alternance et l'enseignement de promotion sociale.

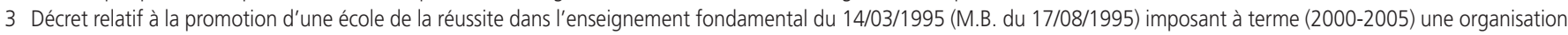
en cycles à l'intérieur desquels l'élève parcourt sa scolarité de manière continue, à son rythme et sans redoublement.

4 Arrêté royal relatif à l'organisation de l'enseignement secondaire du 29/06/1984 (M.B. du 03/08/1984) tel que modifié par A.Gt du 20/06/1994.

5 Décret relatif à l'organisation du premier degré de l'enseignement secondaire du 19/07/2001 (M.B. du 23/08/2001).

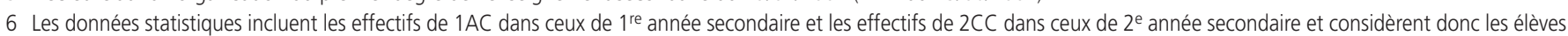
de $1 \mathrm{AC}$ et $2 \mathrm{CC}$ comme redoublants. 


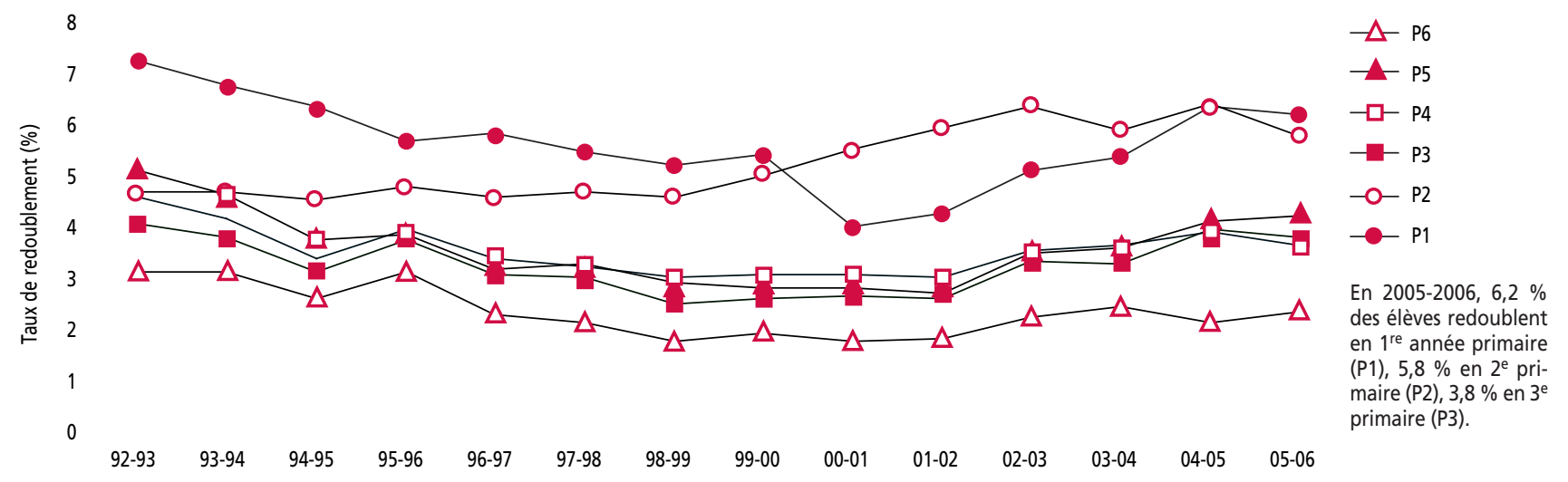

\section{2 Évolution du taux de redoublement dans lenseignement secondaire ordinaire de 1992-1993 à 2005-2006}

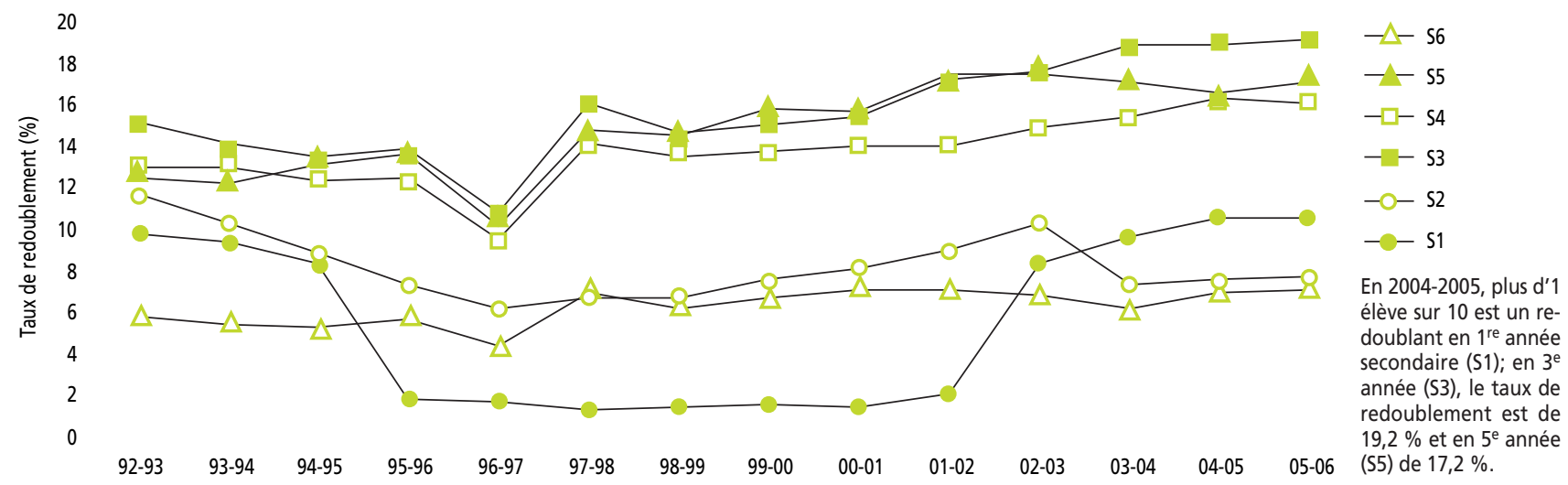

\section{3 Évolution du taux de redoublement en troisième et cinquième secondaire ordinaire, selon la forme d'enseignement suivie de 1992-1993 à 2005-2006}

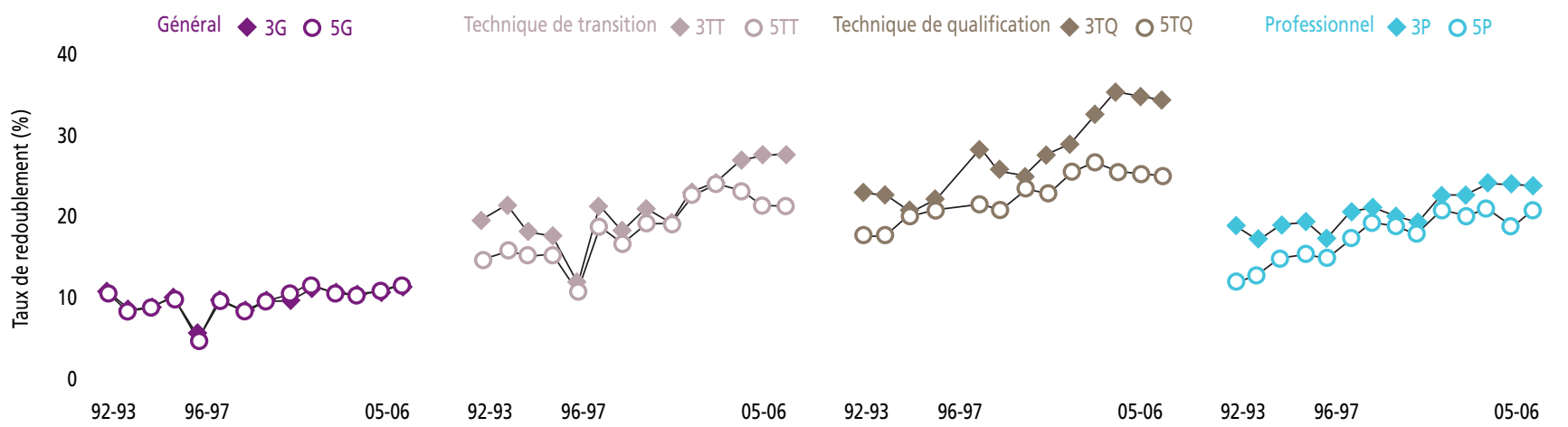

En 2005-2006, on observe des taux de redoublement très différents en $3^{\mathrm{e}}$ année secondaire selon la forme d'enseignement suivie : 11,4 \% dans la forme générale (3G), 26,2 \% en technique (et artistique) de transition (3TT), 32,8 \% en technique (et artistique) de qualification (3TQ) et 23,2 \% dans la forme professionnelle (3P). 
En Communauté française, durant le parcours du primaire ou du secondaire ordinaire, le changement d'établissement est fréquent : en 20052006, il touche $7,5 \%$ des élèves au primaire et $17 \%$ au secondaire, en plus des $2 \%$ concomitants au changement de domicile.

Ces nombreux changements d'établissement produisent, outre des difficultés d'organisation, une ségrégation des publics tout au long du parcours scolaire.

Le redoublement peut expliquer une partie de ces changements d'établissement : le redoublement est en moyenne trois fois plus important pour les élèves changeant d'établissement et ce lien est plus particulièrement accentué en début de cycle ou de degré.

iés en partie au libre choix de l'école par les familles, les changements -d'établissement en cours de scolarité sont fréquents dans l'enseignement en Communauté française. Les données analysées concernent l'enseignement ordinaire de plein exercice, à l'exception des $6^{\mathrm{e}}$ années du primaire et du secondaire, à l'issue desquelles le changement d'établissement en fin d'année scolaire est la règle.

La figure 1 présente les changements d'établissement qui se sont produits entre le 15 janvier 2005 et le 15 janvier 2006 dans l'enseignement ordinaire de plein exercice, qu'ils aient été ou non associés à un changement de domicile ${ }^{1}$. Pour l'ensemble de la population considérée, les changements simultanés de domicile et d'établissement sont de l'ordre de $2 \%$. Dans l'enseignement primaire, en moyenne, 7,5\% des élèves ont changé d'établissement sans changer de domicile et 2,5\% d'élèves ont changé à la fois d'établissement et de domicile. Autrement dit, de la $1^{\text {re }}$ à la $5^{\mathrm{e}}$ primaire, 1 élève sur 10 a changé d'école. Dans l'enseignement secondaire, les mouvements sont plus marqués. Dans le $1^{\text {er }}$ degré (S1 et S2), ce sont les élèves qui ont terminé une deuxième année complémentaire (2CC) qui sont les plus nombreux à poursuivre leur scolarité en changeant d'établissement (43\%) et les élèves qui ont terminé la $1 \mathrm{~A}$ qui en changent le moins (13\%). En moyenne toutefois, le taux de changement au sein du $1^{\text {er }}$ degré est de $16 \%$, et concerne donc 1 élève sur 6 . Dans le $2^{\mathrm{e}}$ degré (S3 et $\mathrm{S} 4$ ), les taux de changement sont assez proches quelle que soit la forme suivie : $19 \%$ des élèves dans ce degré changent d'établissement, avec le taux de changement le plus faible dans l'enseignement général (16\%) et le taux le plus élevé (27\%) dans l'enseignement professionnel. En $5^{\mathrm{e}}$ secondaire, ce sont encore $8 \%$ des élèves qui changent d'établissement à la fin de l'année. Ces changements d'établissement tout au long du parcours scolaire posent la question de l'effectivité du continuum pédagogique et de l'efficacité des orientations proposées.

La figure 2 présente l'écart moyen entre les indices socioéconomiques des établissements ${ }^{2}$ fréquentés successivement par les élèves qui ont changé d'établissement entre le 15 janvier 2005 et le 15 janvier 2006. Un déménagement est généralement l'explication d'un changement d'établissement, c'est pourquoi les données concernent ici les élèves qui ont changé d'établissement sans avoir changé de domicile et dont l'explication doit être trouvée ailleurs. En moyenne, les élèves ayant changé d'école primaire après le 15 janvier 2005 se retrouvent le 15 janvier sui- vant dans une école dont l'indice socioéconomique est plus élevé ${ }^{3}$. On note donc des parcours ascendants dans l'échelle socioéconomique des établissements fréquentés. En revanche, dans l'enseignement secondaire, les élèves ayant changé d'établissement vont, en moyenne, vers un établissement $d^{\prime}$ 'indice socioéconomique plus faible. Dans le $1^{\text {er }}$ degré, l'écart est le plus marqué pour les élèves des années complémentaires (1 AC et $2 \mathrm{CC}$ ) et dans les années d'études ultérieures, pour les élèves de la section de transition. Au secondaire, les changements d'établissement produisent donc des parcours descendants dans l'échelle socioéconomique des établissements fréquentés. Outre l'impact qu'ils peuvent avoir sur l'organisation des établissements, ces mouvements importants de population scolaire caractérisés par des parcours ascendants au primaire et descendants au secondaire, contribuent à la ségrégation des publics scolaires entre établissements.

La figure 3 compare les taux de redoublement ${ }^{4}$ des élèves selon qu'ils changent ou non d'établissement. Ici sont pris en compte les élèves ayant changé d'établissement entre deux années de l'enseignement ordinaire de plein exercice, à l'exception des élèves ayant changé de domicile. A la fin d'une année d'étude effectuée au primaire en 2004-2005, en moyenne $14 \%$ des élèves changeant d'établissement recommencent la même année d'études en 2005-2006 pour $4 \%$ des élèves restés dans le même établissement. Pour les élèves terminant la $1^{\text {re }}$ année primaire, ces rapports atteignent respectivement $21 \%$ et $5 \%$. Dans le $1^{\text {er }}$ degré de I'enseignement secondaire, les taux de redoublement sont assez semblables que les élèves aient changé d'établissement ou pas, sauf pour les élèves ayant terminé la $1 \mathrm{~A}$ en 2004-2005: $28 \%$ de redoublement pour ceux qui ont changé d'établissement en 2005-2006, $8 \%$ pour les autres. Dans le $2^{\mathrm{e}}$ degré, on observe un rapport moyen de 3 à 1 entre les taux de redoublement des élèves selon qu'ils ont changé d'établissement ou pas. En $5^{\mathrm{e}}$ secondaire, le redoublement d'un élève de $5 \mathrm{G}$ est 10 fois plus fréquent s'il change d'établissement, et seulement 5 fois dans les autres formes. Si le redoublement n'est pas la seule cause des changements d'établissement, il semble toutefois en expliquer la plupart, particulièrement aux moments de début de cycle ou de degré (P1, S1, S3 et S5).

De nouvelles dispositions décrétales visant à limiter les changements d'école dans l'enseignement fondamental et au 1er degré du secondaire entreront en application le $1^{\mathrm{er}}$ septembre 2008. L'évolution des données de cet indicateur permettra d'apprécier les effets de ce décret.

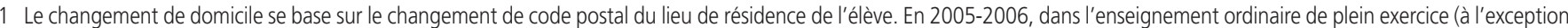
des $6^{e}$ années), on observe qu'un élève sur trois qui a changé de domicile a également changé d'établissement.

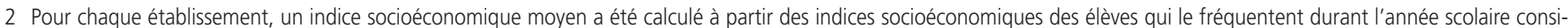
dérée. Plus d'information sur cet indice socioéconomique dans l'indicateur 5.

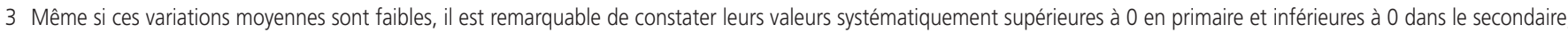
avec de plus une similarité des variations selon les sections en secondaire.

4 Les données statistiques incluent dans les effectifs des redoublants les élèves inscrits dans les années complémentaires d'un cycle ou d'un degré 
15.1 Pourcentages d'élèves ayant changé d'établissement en 2005-2006 (avec ou sans changement de domicile) en fonction de l'année d'études fréquentée en 20042005

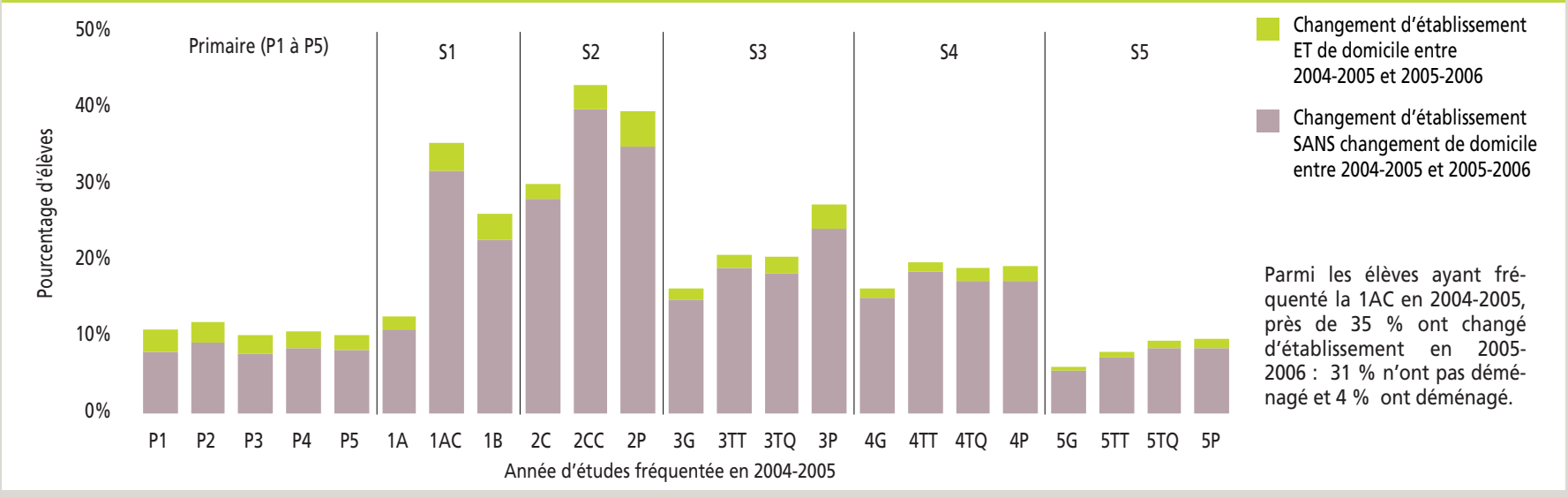

15.2 Écart moyen entre les indices socioéconomiques (ISE) des établissements fréquentés en 2004-2005 et 2005-2006 en fonction de l'année d'études fréquentée en 2004-2005 par les élèves changeant d'établissement (sans changement de domicile)

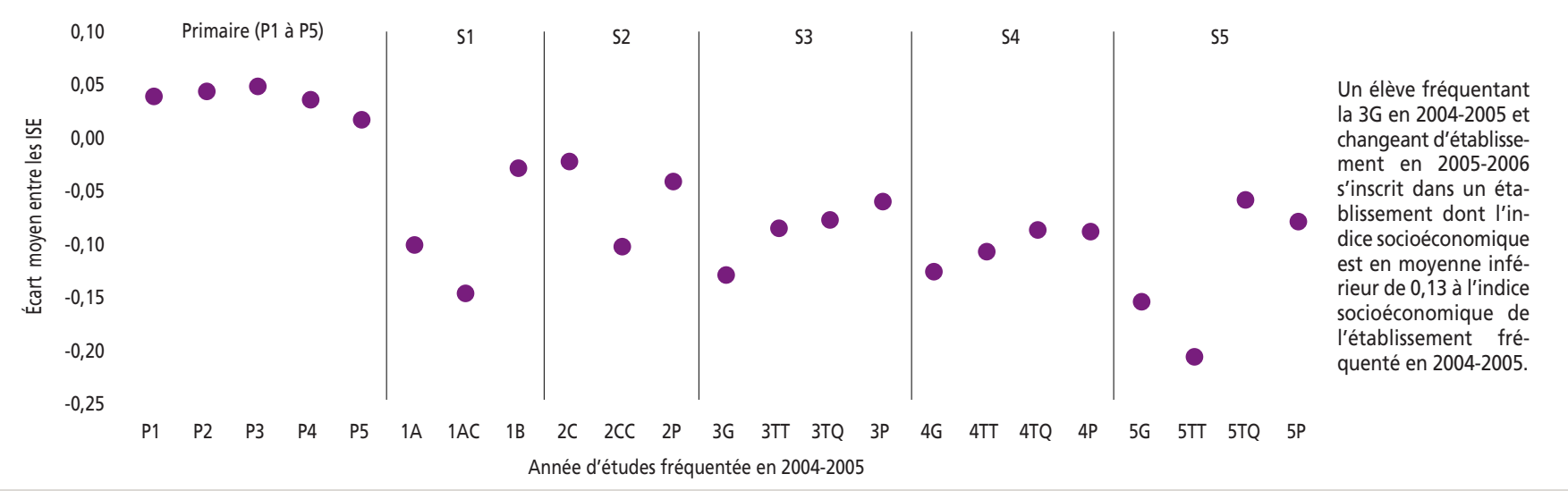

15.3 Taux de redoublement des élèves changeant ou non d'établissement en 2005-2006 (sans changer de domicile) et poursuivant leurs études dans l'enseignement ordinaire de plein exercice, en fonction de l'année d'études fréquentée en 2004-2005

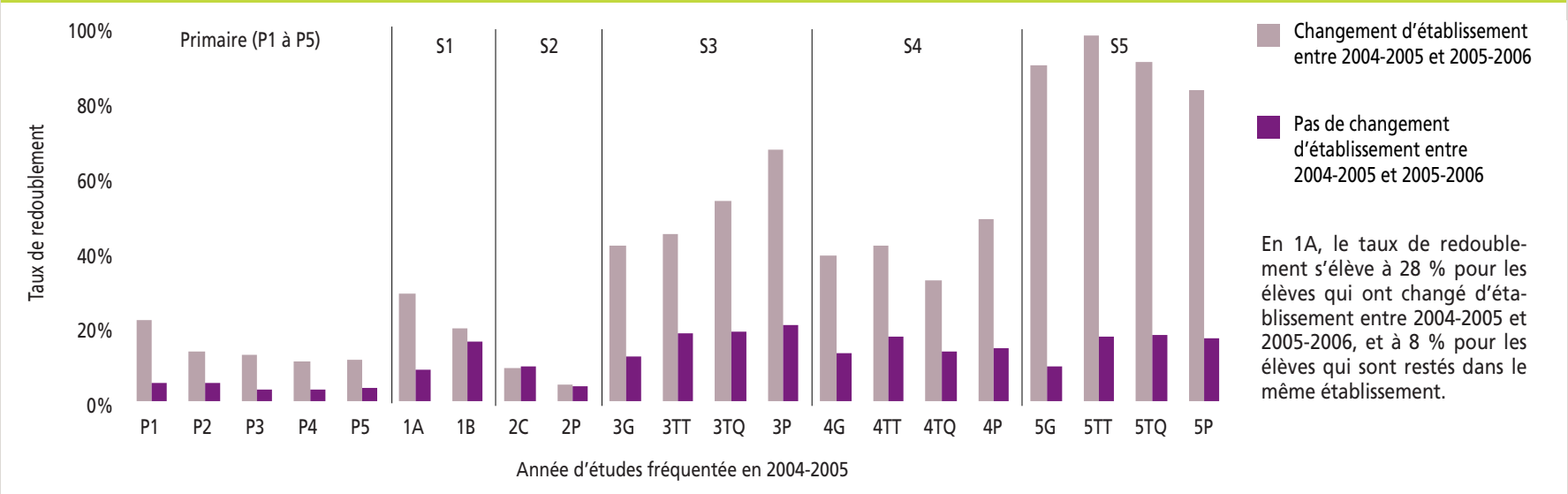


Au terme de l'année scolaire 2004-2005, environ $15 \%$ des élèves en fin de $1^{\text {er }}$ degré ont vu leur année sanctionnée par une attestation de réorientation (attestation $\mathrm{B}$ ). C'est aussi le cas d'environ $10 \%$ des jeunes en cours de $2^{\mathrm{e}}$ degré.

Trois tendances se dessinent concernant les attestations B : celle de l'enseignement professionnel qui en délivre très peu ; la tendance de l'enseignement général qui attribue majoritairement des attestations $B$ incitant l'élève à poursuivre sa scolarité dans la section de qualification ; la tendance de toutes les formes d'enseignement qui octroient peu d'attestations proposant un changement d'option dans la même forme d'enseignement.

D ns l'enseignement secondaire ordinaire, les attestations d'orientation délivrées par le Conseil de classe en fin d'année scolaire sont l'attestation de réussite ( $A O A)$, l'attestation d'échec ( $A O C$ ) et l'attestation de réussite avec restriction ( $A O B)$. Cette dernière vise la réorientation de l'élève en permettant à celui-ci de passer dans l'année supérieure s'il s'oriente vers une autre forme, une autre section ou une autre option. L'élève peut refuser la restriction ou réorientation et choisir de redoubler. Ainsi, l'attestation BG propose à un élève de l'enseignement général de s'orienter vers l'enseignement secondaire technique de transition ou de qualification, ou vers l'enseignement professionnel. L'attestation BT est une restriction sur la section de transition (général et technique). L'attestation $B Q$ dirige l'élève vers l'enseignement professionnel. Enfin, les attestations BA sont des attestations d'orientation vers d'autres options. Ces attestations d'orientation sont délivrées à partir de la $2^{\mathrm{e}}$ année secondaire. Les analyses présentées ici, pour les résultats de l'année scolaire 2004-2005, excluent donc la $1^{\text {re }}$ année. Elles excluent également le $3^{\mathrm{e}}$ degré qui est peu concerné par la réorientation : les jeunes sont censés effectuer tout le degré dans la même section, la même forme et la même option d'enseignement. Seules les attestations de réussite $(A)$ ou d'échec (C) leur sont délivrées ${ }^{1}$.

Les données globales relatives aux attestations révèlent que les attestations $B$ (visant la réorientation) concernent environ $15 \%$ des élèves du $1^{\mathrm{er}}$ degré et $10 \%$ des élèves du $2^{\mathrm{e}}$ degré. La figure 1 détaille, pour chacun des deux degrés, la distribution des attestations $A, B$ et $C$ par année d'études, selon les formes et sections d'enseignement. Trois constats peuvent être faits. Premièrement, le $1^{\text {er }}$ degré de l'enseignement général affiche, en fin de $2^{\mathrm{e}}$ année, le taux d'échec le plus faible, $5,4 \%$ pour les filles et 7,7 \% pour les garçons, ainsi qu'un taux d'attestations d'orientation $B$ de $16,0 \%$ pour les filles et de $19,0 \%$ pour les garçons. Deuxièmement, la figure 1 indique que les attestations $B$ sont encore fréquentes durant le $2^{\mathrm{e}}$ degré de l'enseignement général, du technique de transition et du technique de qualification : dans l'enseignement général, au moins $9,8 \%$ des élèves se voient décerner une attestation $B$ en fin de $3^{\mathrm{e}}$ année et au moins $15,2 \%$ en fin de $4^{\mathrm{e}}$ année ; le technique de qualification affiche des taux moyens (pour les deux sexes) $d^{\prime}$ 'attestations d'orientation $B$ de $11,9 \%$ en $3^{\mathrm{e}}$ et $11,5 \%$ en $4^{\mathrm{e}}$ année ; c'est le technique de transition qui attribue le plus d'attestations d'orientation $B$ (jusqu'à $20,2 \%$ pour les filles de $4^{e}$ année). Troisièmement, la figure 1 met en exergue le peu d'attestations $B$ délivrées en professionnel.
Dans cette forme d'enseignement, seules les attestations BA sont attribuées. Ces restrictions sur l'option concernent 1,9\% des filles et 2,0 \% des garçons qui terminent la $2^{\mathrm{e}}$ année, et entre 1,5 et $2,8 \%$ des élèves en cours de $2^{\mathrm{e}}$ degré. Peu de jeunes de l'enseignement professionnel reçoivent donc une attestation d'orientation B. Cela peut être dû à la " spécialisation technique » de cet enseignement. Le changement d'option s'accompagnerait souvent d'un redoublement pour que l'élève bénéficie de la totalité de sa nouvelle formation professionnelle.

La figure 2 fournit le détail des types d'attestations B délivrées aux $1^{\text {er }}$ et $2^{\mathrm{e}}$ degrés par l'enseignement général, le technique de transition et le technique de qualification ${ }^{2}$. On constate que, quelle que soit l'année d'études, l'enseignement général délivre plus d'attestations BT qui restreignent l'accès à toute la section de transition que d'attestations BG (restriction sur la forme générale seule). Plus généralement, pour toutes les années d'études et toutes les formes d'enseignement, la tendance est à l'attribution d'attestations proposant à l'élève un changement de forme d'enseignement ou même de section. On voit, par exemple, que I'enseignement technique de qualification a tendance à proposer aux élèves des attestations B les incitant à poursuivre leur cursus dans l'enseignement professionnel plutôt que de changer d'option en restant dans la même forme d'enseignement. Les attestations BA, qui proposent un changement d'option, sont donc peu délivrées, sauf pour la $4^{\mathrm{e}}$ année du général au terme de laquelle $44,5 \%$ des élèves recevant une attestation $B$ se voient suggérer un changement d'option de base. Le début $d u 3^{e}$ degré semble ainsi être un lieu où s'affine encore l'orientation des élèves de l'enseignement général.

\section{Glossaire}

AOA : Attestation d'Orientation A ou attestation de réussite de l'année d'études délibérée.

$A O B$ : Attestation d'Orientation $\mathrm{B}$ ou attestation de réussite avec, pour le passage à l'année supérieure, restriction sur le choix de la section, de la forme ou de l'option.

$A O C$ : Attestation d'Orientation $C$ ou attestation d'échec.

$B G$ : $\quad A O B$ dont la restriction porte sur l'enseignement général.

$B T$ : $A O B$ avec restriction sur toute la section de transition.

$B Q$ : $\quad A O B$ orientant l'élève vers l'enseignement professionnel.

$B A$ : $\quad A O B$ « Autre » qui propose à l'élève un changement d'option.

\footnotetext{
1 Pour les statistiques de réussite en fin de $3^{e}$ degré (à l'exclusion des $7^{\text {es }}$ ), on consultera les indicateurs relatifs à la certification en $6^{\mathrm{e}}$ secondaire qui présentent aussi les statistiques d'échecs en $5^{\mathrm{e}}$.

2 L'enseignement professionnel ne délivrant que des attestations BA, il n'est pas repris ici.
} 
16.1 Répartition des trois types d'attestations d'orientation (A, B ou C) par degré de l'enseignement secondaire, année d'études et sexe - Année de certification 2005

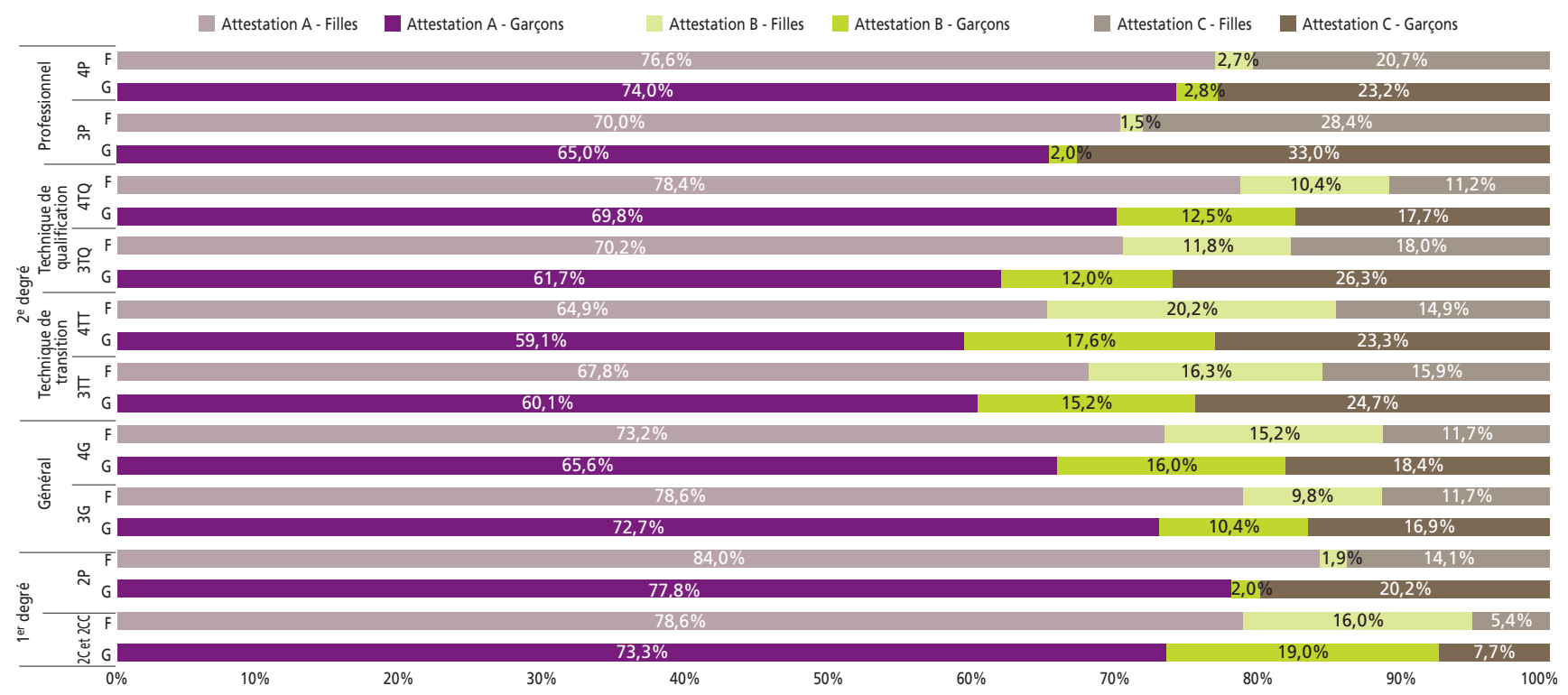

Parmi les attestations d'orientation en $4^{e}$ année de l'enseignement technique de transition, 20,2 \% et 17,6 \% sont des attestations B délivrées respectivement aux filles et aux garçons.

16.2 Répartition des différents types d'attestations d'orientation B (BG, BT, BQ, BA) délivrées aux $1^{\text {er }}$ et $2^{\mathrm{e}}$ degrés, selon la forme d'enseignement, l'année d'études et le sexe-Année de certification 2005

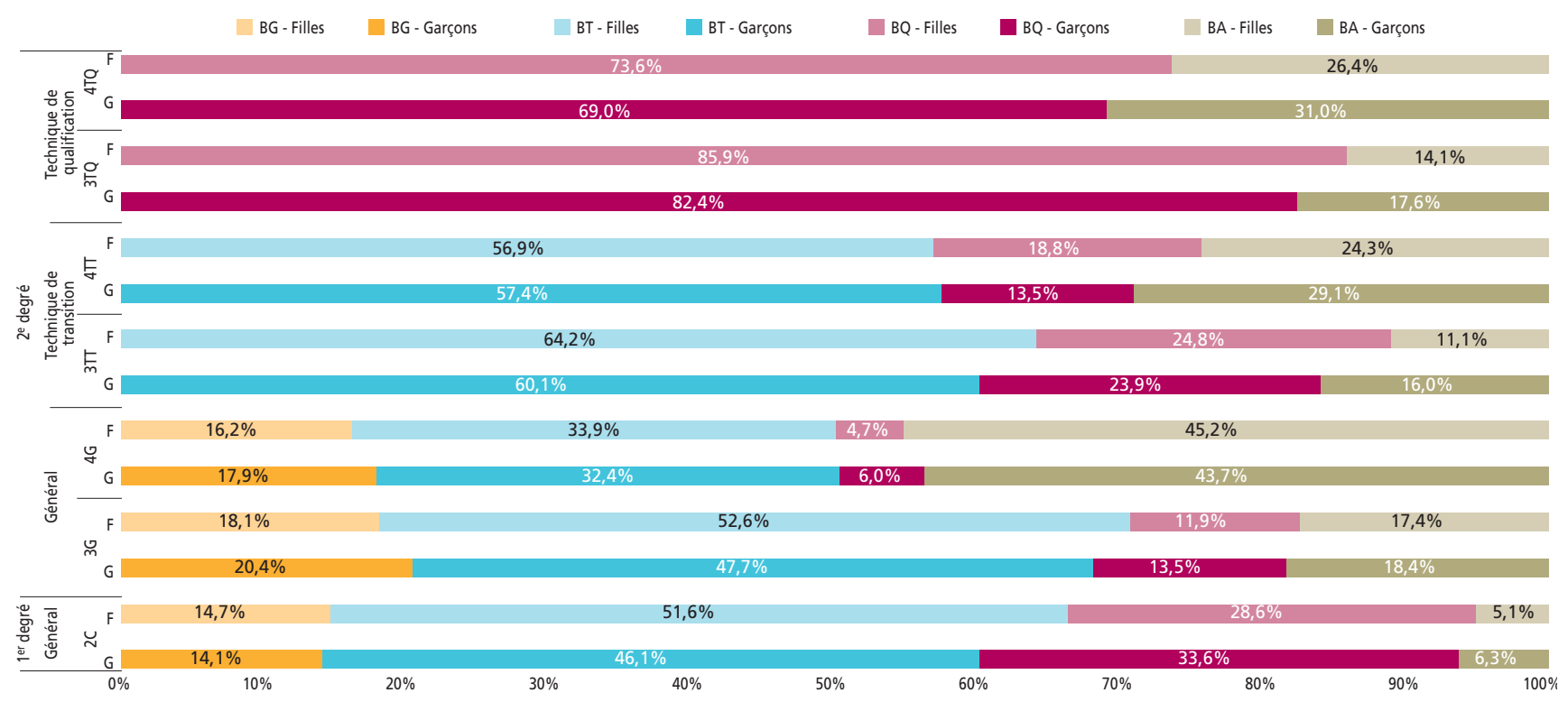

Parmi les attestations d'orientation B délivrées aux garçons de 4 e année technique de transition, $57,4 \%$ sont des attestations avec restriction pour l'enseignement de transition (BT), 13,5\% orientent l'élève vers l'enseignement professionnel (BQ) et 29,1 \% lui proposent un changement d'option (BA). 
Les élèves inscrits en $1^{\text {re }}$ année A durant l'année scolaire 2003-2004 sont plus de $60 \%$ à poursuivre, 2 années plus tard, leurs études secondaires dans l'enseignement ordinaire de transition.

Inversement, les élèves inscrits en $1^{\text {re }}$ année B en 2003-2004 sont près de $70 \%$ à fréquenter l'enseignement ordinaire qualifiant en 2005-2006.

$E^{2}$ 2003-2004, 55047 élèves débutaient l'enseignement secondaire de plein exercice en Communauté française. Plus de $90 \%$ d'entre eux (49 625 élèves) étaient en $1^{\text {re }}$ année $A$ (1A), les autres (5 422 élèves) étaient en 1re année $B$ (1B). Le présent indicateur retrace les parcours scolaires de ces élèves sur 3 années scolaires, de 2003-2004 à 2005-2006.

La figure 1 décrit les parcours de la cohorte des 49625 élèves de $1 \mathrm{~A}$ en 2003-2004 et indique d'abord (ligne de total) que, I'année suivante, $80,9 \%$ de ces élèves passent en $2^{\mathrm{e}}$ année commune (2C) et qu'ils poursuivent majoritairement dans l'enseignement général. En effet, en 2005-2006, $54 \%$ des élèves de la cohorte sont en $3^{\mathrm{e}}$ générale (3G). Ensuite, la figure 1 montre que 9,6 \% des élèves de 1A en 2003-2004 effectuent une $1^{\text {re }}$ année complémentaire (1AC) en 2004-2005 et qu'ils ont majoritairement tendance, I'année suivante, à rejoindre la $2 \mathrm{C}(6,7 \%$ de la cohorte). Enfin, le passage direct de la $1 \mathrm{~A}$ en 2003-2004 à la $2^{\mathrm{e}}$ année professionnelle (2P) en 2004-2005 se fait pour 6,0\% des élèves, dont $5,6 \%$ poursuivent l'enseignement professionnel (3P) durant la troisième année considérée. La figure 1 révèle aussi que moins de $0,6 \%$ et moins de $0,2 \%$ des élèves de $1 \mathrm{~A}$ rejoignent respectivement l'enseignement en alternance (CEFA) et l'enseignement spécialisé au cours des 2 années scolaires suivantes.

La figure 2 présente les parcours des 5422 élèves inscrits en 1B en 2003-2004. On note, premièrement, que la grande majorité $(70,7 \%)$ passe en $2 \mathrm{P}$ l'année suivante et que leurs parcours se diversifient peu par la suite : $61,7 \%$ de la cohorte se trouvent en 3P en 2005-2006 et 5,3\% dans l'enseignement en alternance. Deuxièmement, $15,3 \%$ des élèves rejoignent la $1 \mathrm{~A}$ au terme de la $1 \mathrm{~B}$ et un total de $10,4 \%$ de la cohorte poursuit alors en $2 \mathrm{C}$ ou en $1 \mathrm{AC}$ en 2005-2006. Troisièmement, un total de $4,9 \%$ des élèves de $1 \mathrm{~B}$ rejoint l'enseignement spécialisé, soit en 2004-2005, soit en 2005-2006.

La figure 3 montre comment filles et garçons, inscrits en 2003-2004 en $1 A$ et en $1 B$, se répartissent dans l'enseignement en 2005-2006. La ré- partition est organisée par sections et types d'enseignement, auxquels $s^{\prime}$ ajoutent 2 catégories : les élèves toujours présents au $1^{\text {er }}$ degré ( 2 ans après leur entrée dans le secondaire) et la catégorie "Sorties". La comparaison des parcours selon l'année d'études de départ (1A ou 1B) met en exergue plusieurs éléments. Premièrement, deux tendances majoritaires se dessinent, celle des élèves de $1 \mathrm{~A}$ à poursuivre leur cursus en section de transition (64,5\% des filles et $54,8 \%$ des garçons), et celle des élèves de $1 \mathrm{~B}$ à poursuivre leur scolarité en section de qualification ( $70,8 \%$ des filles et $63,9 \%$ des garçons). Deuxièmement, on note que le maintien de l'élève durant 3 années au sein du $1^{\text {er }}$ degré concerne 12,9\% des filles et $18,0 \%$ des garçons de $1 \mathrm{~A}, 15,2 \%$ des filles et $12,7 \%$ des garçons de $1 \mathrm{~B}$. Troisièmement, les élèves de $1 \mathrm{~A}$ dirigés vers I'enseignement en alternance ou vers l'enseignement spécialisé sont extrêmement rares. Par contre, parmi les élèves ayant fréquenté la 1B en 2003-2004, I'enseignement en alternance accueille 4,5\% des filles et $6,6 \%$ des garçons, et l'enseignement spécialisé 3,0 \% des filles et 5,6 \% des garçons. Quatrièmement, ce sont plus souvent les élèves de $1 \mathrm{~B}$ qui, 2 ans plus tard, se trouvent dans la catégorie "Sorties ", celle des élèves qui ont quitté l'enseignement scolaire.

La figure 3 permet enfin, pour chacune des 2 années d'études de départ, une comparaison des parcours des garçons et des filles. Ainsi les garçons de $1 \mathrm{~A}$ sont-ils un peu plus nombreux que les filles à rejoindre la section de qualification de l'enseignement secondaire ordinaire de plein exercice (23,8 \% des garçons contre $20,4 \%$ des filles). Par contre, à partir de la $1 \mathrm{~B}$, ce sont les filles qui alimentent davantage cette section : $70,8 \%$ contre $63,9 \%$ des garçons. Cette moindre participation des garçons à la section de qualification de l'enseignement ordinaire de plein exercice se compense, non par une plus grande insertion dans la section de transition, mais par une participation relativement plus importante, en 2005-2006, à l'enseignement en alternance, à l'enseignement spécialisé et à la catégorie « Sorties ". 
17.1 Parcours des élèves de tre année A (1A) de l'enseignement secondaire ordinaire de plein exercice - Années scolaires 2003-2004 à 2005-2006

\begin{tabular}{|c|c|c|c|c|}
\hline Année scolaire 2003-2004 & Année scolaire 2004-2005 & Année scolaire 2005-2006 & Nombre d'élèves dans le parcours & Pourcentage de la cohorte \\
\hline $1 \mathrm{~A}$ & $2 \mathrm{C}$ & $3 G$ & 26819 & $54,0 \%$ \\
\hline $1 \mathrm{~A}$ & $2 \mathrm{C}$ & 3TQ & 4962 & $10,0 \%$ \\
\hline $1 \mathrm{~A}$ & $2 \mathrm{C}$ & $2 \mathrm{CC}$ & 3951 & $8,0 \%$ \\
\hline $1 \mathrm{~A}$ & $2 \mathrm{C}$ & 3TT & 2635 & $5,3 \%$ \\
\hline $1 \mathrm{~A}$ & $2 \mathrm{C}$ & $3 P$ & 1688 & $3,4 \%$ \\
\hline $1 \mathrm{~A}$ & $2 \mathrm{C}$ & Ens. en alternance & 62 & $0,1 \%$ \\
\hline $1 \mathrm{~A}$ & $2 \mathrm{C}$ & Ens. spécialisé & 6 & $0,0 \%$ \\
\hline \multicolumn{3}{|c|}{ Nombre total d'élèves passant de $1 \mathrm{~A}$ en $2 \mathrm{C}$} & 40123 & $80,9 \%$ \\
\hline $1 \mathrm{~A}$ & $1 \mathrm{AC}$ & $2 \mathrm{C}$ & 3346 & $6,7 \%$ \\
\hline $1 \mathrm{~A}$ & $1 \mathrm{AC}$ & 3P & 1024 & $2,1 \%$ \\
\hline $1 \mathrm{~A}$ & $1 \mathrm{AC}$ & 3TQ & 341 & $0,7 \%$ \\
\hline $1 \mathrm{~A}$ & $1 \mathrm{AC}$ & Ens. en alternance & 33 & $0,1 \%$ \\
\hline $1 \mathrm{~A}$ & $1 \mathrm{AC}$ & Ens. spécialisé & 7 & $0,0 \%$ \\
\hline \multicolumn{3}{|c|}{ Nombre total d'élèves passant de $1 \mathrm{~A}$ en $1 \mathrm{AC}$} & 4751 & $9,6 \%$ \\
\hline $1 \mathrm{~A}$ & $2 \mathrm{P}$ & 3P & 2759 & $5,6 \%$ \\
\hline $1 \mathrm{~A}$ & $2 \mathrm{P}$ & Ens. en alternance & 149 & $0,3 \%$ \\
\hline $1 \mathrm{~A}$ & $2 \mathrm{P}$ & $2 \mathrm{C}$ & 76 & $0,2 \%$ \\
\hline $1 \mathrm{~A}$ & $2 P$ & Ens. spécialisé & 12 & $0,0 \%$ \\
\hline \multicolumn{3}{|c|}{ Nombre total d'élèves passant de $1 \mathrm{~A}$ en $2 \mathrm{P}$} & 2996 & $6,0 \%$ \\
\hline $1 \mathrm{~A}$ & Ens. spécialisé & & 34 & $0,1 \%$ \\
\hline $1 \mathrm{~A}$ & Ens. en alternance & & 10 & $0,0 \%$ \\
\hline $1 \mathrm{~A}$ & Sorties & & 1711 & $3,4 \%$ \\
\hline \multicolumn{3}{|c|}{ Total de la cohorte de 1 A en 2003-2004 } & 49625 & $100,0 \%$ \\
\hline
\end{tabular}

Parmi les élèves inscrits en $1^{\text {re }}$ année $A(1 A)$ en 2003-2004, 80,9 \% passent en $2^{\mathrm{e}}$ année Commune (2C) en 2004-2005.

17.2. Parcours des élèves de $1^{r e}$ année B (1B) de lenseignement secondaire ordinaire de plein exercice - Années scolaires 2003-2004 à 2005-2006

\begin{tabular}{|c|c|c|c|c|}
\hline Année scolaire 2003-2004 & Année scolaire $2004-2005$ & Année scolaire 2005-2006 & Nombre d'élèves dans le parcours & Pourcentage de la cohorte \\
\hline 1B & 2P & 3P & 3347 & $61,7 \%$ \\
\hline 1B & $2 \mathrm{P}$ & Ens. en alternance & 289 & $5,3 \%$ \\
\hline 1B & $2 \mathrm{P}$ & Ens. spécialisé & 96 & $1,8 \%$ \\
\hline 1B & $2 \mathrm{P}$ & $2 \mathrm{C}$ & 37 & $0,7 \%$ \\
\hline 1B & $2 \mathrm{P}$ & $2 \mathrm{CC}$ & 37 & $0,7 \%$ \\
\hline 1B & $2 \mathrm{P}$ & $2 \mathrm{P}$ & 30 & $0,6 \%$ \\
\hline \multicolumn{3}{|c|}{ Nombre d'élèves passant de 1B en 2P } & 3836 & $70,7 \%$ \\
\hline 1B & $1 \mathrm{~A}$ & $2 \mathrm{C}$ & 504 & $9,3 \%$ \\
\hline 1B & $1 \mathrm{~A}$ & $3 \mathrm{P}$ & 206 & $3,8 \%$ \\
\hline 1B & $1 \mathrm{~A}$ & $2 \mathrm{P}$ & 64 & $1,2 \%$ \\
\hline 1B & $1 \mathrm{~A}$ & $1 \mathrm{AC}$ & 57 & $1,1 \%$ \\
\hline \multicolumn{3}{|c|}{ Nombre d'élèves passant de $1 \mathrm{~B}$ en $1 \mathrm{~A}$} & 831 & $15,3 \%$ \\
\hline 1B & $3 \mathrm{P}$ & 4P & 27 & $0,5 \%$ \\
\hline \multicolumn{3}{|c|}{ Nombre d'élèves passant de $1 B$ en $3 P$} & 27 & $0,5 \%$ \\
\hline 1B & Ens. spécialisé & & 167 & $3,1 \%$ \\
\hline 1B & Ens. en alternance & & 13 & $0,2 \%$ \\
\hline 1B & Sorties & & 548 & $10,1 \%$ \\
\hline \multicolumn{3}{|c|}{ Total de la cohorte de 1B en 2003-2004 } & 5422 & $100,0 \%$ \\
\hline
\end{tabular}

Parmi les élèves inscrits en $1^{\text {re }}$ année B (1B) en 2003-2004, 61,7 \% poursuivent, sans redoublement, leur scolarité les 2 années suivantes dans l'enseignement professionnel.

17.3 Répartition, selon le sexe, des élèves de 1A et de 1B par degré, section et type d'enseignement dans lesquels ils sont inscrits 2 ans plus tard - Années scolaires 2003-2004 et 2005-2006

$80 \%$

$70 \%$

$60 \%$

$50 \%$

$40 \%$

$30 \%$

$20 \%$

$10 \%$

$0 \%$

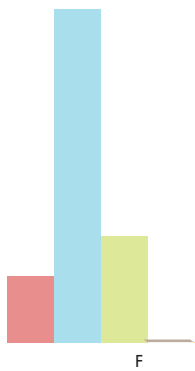

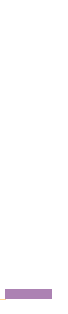

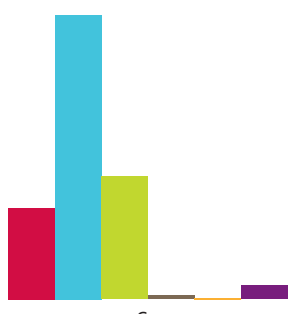

Année scolaire 2003-2004
Année scolaire 2005-2006

1er degré

Section de transition

- Section de qualification

Enseignement en alternance

Enseignement spécialisé

Autres (dont "non scolarisés")

Parmi les filles inscrites en $1^{\text {re }}$ année $B(1 B), 70,8 \%$ se trouvent en section de qualification 2 ans plus tard. C'est le cas de 63,9\% des garçons. 
C'est à l'âge de 7 ans qu'a lieu le maximum d'entrées dans l'enseignement spécialisé : $14 \%$.

$70 \%$ des entrées dans l'enseignement spécialisé se font par le niveau primaire et $21 \%$ par le niveau secondaire.

$30 \%$ des sorties de l'enseignement spécialisé se produisent à 13 et 14 ans, donnant lieu à une réorientation vers l'enseignement ordinaire.

$43 \%$ des sorties de l'enseignement spécialisé ont lieu après 17 ans, et correspondent à des sorties définitives de l'enseignement obligatoire.

Entre 2004-2005 et 2005-2006, $81 \%$ des élèves de l'enseignement spécialisé sont restés dans le même type d'enseignement, 5 \% ont changé de type d'enseignement spécialisé, $6 \%$ se sont réorientés vers l'enseignement ordinaire et $8 \%$ sont sortis définitivement de l'enseignement obligatoire.

et indicateur étudie les flux d'entrée et de sortie de l'enseignement spécialisé ainsi que l'ampleur des réorientations au sein de l'enseignement spécialisé et vers l'enseignement ordinaire. Seuls les élèves domiciliés en Belgique sont pris en considération dans cet indicateur.

Les figures 1 et 2 présentent la répartition des entrées ${ }^{1}$ dans l'enseignement spécialisé en 2005-2006, en fonction de l'âge des élèves et selon le niveau et le type ${ }^{2} \mathrm{~d}^{\text {enseignement fréquenté. }}$

La figure 1 montre que le maximum d'entrées, $14 \%$, s'observe à l'âge de 7 ans et que près de trois-quart des entrées se déroulent entre 6 et 12 ans. Les entrées dans l'enseignement spécialisé se font donc essentiellement au niveau primaire (70\%) mais, comme la figure 2 l'indique, $9 \%$ des entrées se font dès le niveau maternel et $21 \%$ des entrées ont encore lieu au niveau secondaire.

En observant les entrées par type d'enseignement spécialisé, on constate que $35 \%$ des élèves entrent dans l'enseignement de type 8 (troubles instrumentaux), au niveau primaire, $35 \%$ entrent dans le type 1 (arriération mentale légère), dont $22 \%$ au niveau primaire et $13 \%$ au niveau secondaire, $14,4 \%$ entrent dans le type 3 (troubles caractériels et/ou de personnalité) et 7,8 \% entrent dans le type 2 (arriération mentale modérée ou sévère); le reste des élèves $(15,6 \%)$ entrent dans les autres types d'enseignement.

Les figures 3 et 4 présentent la répartition des sorties ${ }^{3}$ de l'enseignement spécialisé en fin d'année scolaire 2004-2005. La figure 3 indique ces sorties en fonction de l'âge des élèves tandis que la figure 4 montre ces sorties par "destination» de sortie.

Dans la tranche d'âge des élèves en obligation scolaire, c'est à 13 et 14 ans qu'a lieu le maximum des sorties de l'enseignement spécialisé, $30 \%$, dont les deux-tiers sont des sorties de l'enseignement de type 8 (troubles instrumentaux) ${ }^{4}$, tandis que $14 \%$ des élèves sortent de l'enseignement spécialisé entre 15 et 17 ans. Il faut en outre noter que $43 \%$ des sorties de l'enseignement spécialisé se produisent après l'âge de 17 ans.
En étudiant les «destinations» de sortie de l'enseignement spécialisé, on observe que $57 \%$ des sorties de l'enseignement spécialisé sont des sorties définitives de l'enseignement obligatoire ${ }^{5}$ tandis que $37 \%$ sont des sorties vers l'enseignement secondaire ordinaire (le reste, soit $6 \%$, s'oriente vers l'enseignement primaire ordinaire).

La figure 5 décrit le parcours des 27667 élèves (domiciliés en Belgique) inscrits dans l'enseignement spécialisé en 2004-2005, répartis dans les 8 types d'enseignement (axe des ordonnées). Elle indique la "position» de ces élèves en 2005-2006 (axe des abscisses), soit dans un des types d'enseignement spécialisé, soit dans l'enseignement ordinaire, soit sortis de l'enseignement obligatoire.

II apparaît ainsi que $81 \%$ des élèves de l'enseignement spécialisé sont restés, d'une année à l'autre, dans le même type d'enseignement tandis que $5 \%$ d'entre eux, tout en restant dans le spécialisé, ont changé de type d'enseignement, la part la plus importante $(1,7 \%)$ étant la réorientation du type 8 (troubles instrumentaux) vers le type 1 (arriération mentale légère $)^{6}$. En ce qui concerne les autres élèves, $6 \%$ ont été réorientés vers l'enseignement ordinaire (essentiellement vers le niveau secondaire) et $8 \%$ sont sortis définitivement de l'enseignement obligatoire.

\section{Glossaire}

Types d'enseignement spécialisé :

Type 1 : arriération mentale légère

Type 2 : arriération mentale modérée ou sévère

Type 3 : troubles caractériels et/ou de personnalité

Type 4 : déficiences physiques

Type 5 : maladies

Type 6 : déficiences visuelles

Type 7 : déficiences auditives

Type 8 : troubles instrumentaux

1 En 2005-2006, 4218 élèves entrent pour la première fois dans l'enseignement spécialisé.

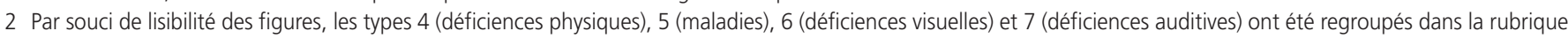
"autres" ; leurs effectifs cumulés représentent moins de $10 \%$ du total des effectifs de l'enseignement spécialisé.

3 En fin d'année scolaire 2004-2005, 3885 élèves sortent définitivement de l'enseignement spécialisé.

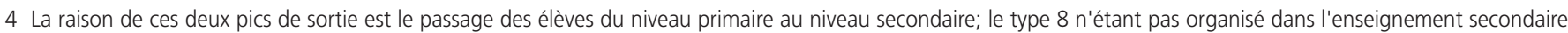
spécialisé, ce passage se traduit par un transfert vers l'enseignement secondaire ordinaire.

5 Enseignement secondaire en alternance (CEFA) inclus.

6 Passage du niveau primaire au niveau secondaire. 
18.1 Répartition des entrées dans le spécialisé par âge et type d'enseignement - Année scolaire 2005-2006

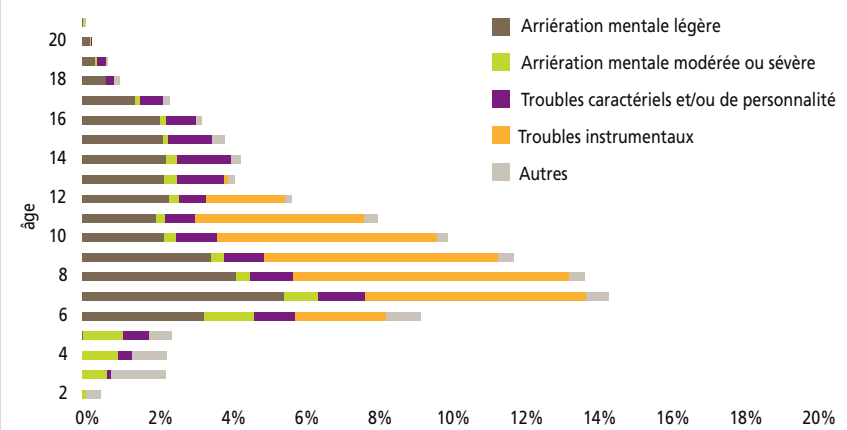

$14 \%$ des entrées se font à l'âge de 7 ans.

18.3 Répartition des sorties du spécialisé par âge et type d'enseignement - Fin d'année scolaire 2004-2005

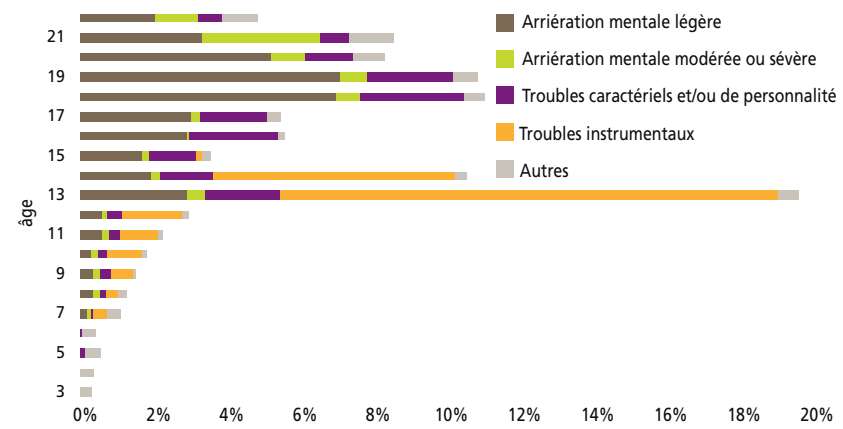

Près de $20 \%$ des sorties ont lieu à 13 ans.
18.2 Répartition des entrées dans le spécialisé par niveau et type d'enseignement - Année scolaire 2005-2006

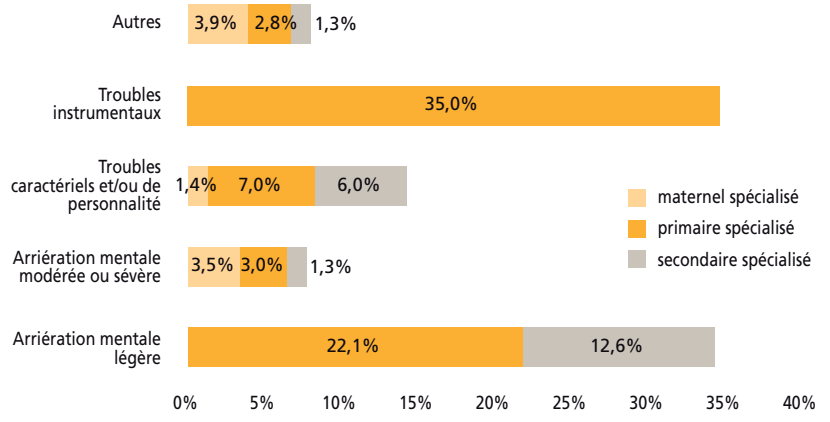

$70 \%$ des entrées se font au niveau primaire, dont la moitié dans le type 8 (troubles instrumentaux).

\subsection{Répartition des udestinations» de sortie du spécialisé par type d'enseignement - Année scolaire 2005-2006}

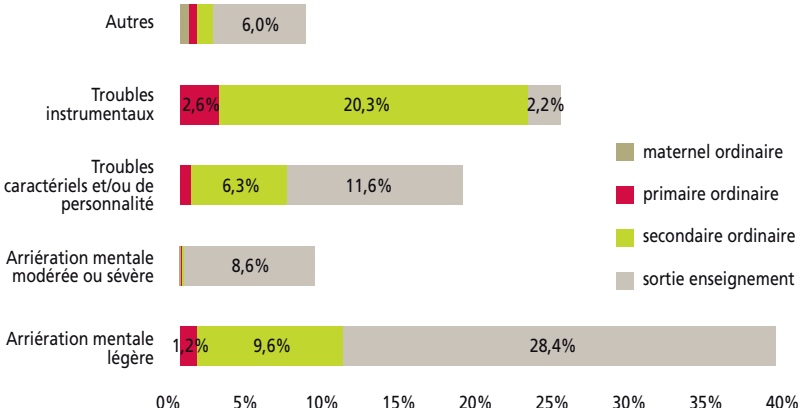

$57 \%$ des sorties sont des sorties définitives de l'enseignement obligatoire, le reste se dirige essentiellement vers le secondaire ordinaire.

\subsection{Transition des élèves inscrits dans l'enseignement spécialisé en 2004-2005 vers l'année scolaire 2005-2006}

\begin{tabular}{|c|c|c|c|c|c|c|c|c|c|c|c|}
\hline type 8 & 477 & 28 & 132 & 8 & 6 & & 3 & 4690 & 100 & 790 & 84 \\
\hline type 7 & 6 & 4 & 1 & 3 & 2 & 1 & 513 & 13 & 8 & 11 & 50 \\
\hline type 6 & 1 & 3 & & 1 & & 206 & & 1 & 4 & 0 & 18 \\
\hline type 5 & 6 & 10 & 23 & 3 & 68 & & & 34 & 10 & 16 & 40 \\
\hline type 4 & 3 & 13 & 1 & 1302 & 2 & 2 & 1 & 6 & 1 & 11 & 127 \\
\hline type 3 & 139 & 45 & 3095 & 3 & 5 & & 2 & 25 & 27 & 246 & 449 \\
\hline type 2 & 66 & 3661 & 21 & 15 & 3 & & 2 & 4 & 3 & 4 & 333 \\
\hline type 1 & 8801 & 129 & 176 & 6 & 2 & & 4 & 35 & 45 & 373 & 1105 \\
\hline & type 1 & type 2 & type 3 & type 4 & type 5 & type 6 & type 7 & type 8 & $\begin{array}{l}\text { fondamental } \\
\text { ordinaire }\end{array}$ & $\begin{array}{l}\text { secondaire } \\
\text { ordinaire }\end{array}$ & $\begin{array}{c}\text { sortie } \\
\text { enseignement }\end{array}$ \\
\hline
\end{tabular}

Parmi les 6318 élèves présents dans le spécialisé de type 8 (troubles instrumentaux) en 2004-2005, 4690 d'entre eux y sont restés en 2005-2006, 790 sont passés dans le secondaire ordinaire et 477 sont passés dans le spécialisé de type 1 (arriération mentale légère). 
Le taux d'accès à l'enseignement supérieur est abordé sous deux angles.

Dans la première approche, le utaux d'accès à l'enseignement supérieur des élèves présents à 17 ans dans l'enseignement secondaire ordinaire de plein exercice" de la génération 1983 s'élève à $56 \%$, avec un écart important entre les hommes (51\%) et les femmes (61 \%) et d'importants écarts selon la forme d'enseignement secondaire fréquentée (91\% pour les élèves du secondaire général, $59 \%$ pour le technique de transition, $37 \%$ pour le technique de qualification et $4 \%$ pour le professionnel).

La seconde approche s'intéresse au utaux d'accès à l'enseignement supérieur des détenteurs du CESS de l'enseignement secondaire ordinaire de plein exercice» délivré en fin d'année 2002-2003, et ne tient donc compte que des élèves du secondaire ayant obtenu le titre donnant accès au supérieur. Ce taux d'accès est de $75 \%$, avec un urattrapage» des hommes, les taux d'accès respectifs des hommes et des femmes étant cette fois de $72 \%$ et 78 \%.

e taux d'accès à l'enseignement supérieur est abordé sous deux an-gles, chacun apportant un éclairage spécifique sur l'entrée des élèves de l'enseignement secondaire dans l'enseignement supérieur.

La première approche présente le suivi de la génération des enfants nés en 1983, en Belgique ou à l'étranger, qui, à l'âge de 17 ans, sont présents dans l'enseignement secondaire ordinaire de plein exercice ${ }^{1}$ en Communauté française (en 2000-2001). La figure 1 montre la proportion de cette génération qui accède à l'enseignement supérieur. La cohorte a été subdivisée selon le sexe et la forme d'enseignement secondaire choisie par l'élève au moment de l'observation. Il faut noter que les élèves de 17 ans ainsi observés peuvent se trouver non seulement en $6^{\mathrm{e}}$ année, ou parfois en $7^{\mathrm{e}}$ année, mais aussi en $5^{\mathrm{e}}, 4^{\mathrm{e}}$ ou même en $3^{\mathrm{e}}$ année de l'enseignement secondaire ordinaire. Ainsi définie, la génération 1983 entre dans l'enseignement supérieur ${ }^{2}$ à partir de l'année académique 20012002 et les observations s'étendent jusqu'à l'année 2005-2006, c'est-àdire jusqu'à l'âge de 22 ans inclus ${ }^{3}$. L'enseignement supérieur a pour sa part été subdivisé en universités, supérieur hors universités (SHU) de type long et SHU de type court.

La figure 1 montre que le «taux d'accès à l'enseignement supérieur des élèves présents à 17 ans dans l'enseignement secondaire ordinaire de plein exercice» est au total de $56 \%(21,4 \%$ à l'université, $6 \%$ dans le SHU de type long et $28,6 \%$ dans le SHU de type court). Il s'élève cependant à $61 \%$ chez les femmes et n'est que de $51 \%$ chez les hommes. Selon la forme d'enseignement secondaire fréquentée par les élèves, le taux d'accès à l'enseignement supérieur varie également. Ainsi, $91 \%$ des élèves du secondaire général accèdent à l'enseignement supérieur, $56 \%$ se dirigeant vers l'enseignement supérieur de niveau universitaire (universités et SHU de type long) et $35 \%$ vers le supérieur de type court. Le taux $d$ 'accès des élèves provenant du secondaire technique de transition est par contre seulement de $59 \%$, dont $17 \%$ s'orientent vers le supérieur de niveau universitaire et $42 \%$ vers le supérieur de type court. Les élèves issus du secondaire technique de qualification accèdent à l'enseignement supérieur à raison de $37 \%$, dont moins de $3 \%$ se dirigent vers le supé- rieur de niveau universitaire et quelque $34 \%$ vers le supérieur de type court. Quant au taux d'accès à l'enseignement supérieur, limité au type court, des élèves provenant du secondaire professionnel, il n'est que d'environ $4 \%$.

La seconde approche consiste à évaluer la proportion d'élèves détenteurs du CESS qui décident d'entrer dans l'enseignement supérieur. Les élèves choisis pour la figure 2 ont obtenu leur CESS en 2003 dans l'enseignement secondaire ordinaire de plein exercice en Communauté française. Ils ont été subdivisés selon le sexe et la forme d'enseignement secondaire fréquentée. L'entrée dans l'enseignement supérieur de ces élèves a lieu à partir de l'année académique 2003-2004' . Comme précédemment, l'enseignement supérieur a été subdivisé en universités, SHU de type long et SHU de type court.

Tout à fait logiquement, puisqu'on ne tient compte que des élèves du secondaire ayant obtenu le droit d'accéder au supérieur, la figure 2 présente des taux supérieurs à la première approche. Ainsi, le «taux d'accès à l'enseignement supérieur des détenteurs du CESS de l'enseignement secondaire ordinaire de plein exercice» s'élève au total à $75 \%$ ( $28,3 \%$ à l'université, $7,6 \%$ dans le SHU de type long et 39,3\% dans le SHU de type court). Il est de $78 \%$ chez les femmes et de $72 \%$ chez les hommes. Les détenteurs du CESS délivré par l'enseignement secondaire général sont $99 \%$ à accéder au supérieur, dont $60 \%$ se dirigent vers le supérieur de niveau universitaire et $39 \%$ vers le supérieur de type court. Le taux d'accès des élèves détenteurs d'un CESS du secondaire technique de transition est, quant à lui, de $78 \%$, dont $22 \%$ s'orientent vers le supérieur de niveau universitaire et $56 \%$ vers le supérieur de type court. Le taux d'accès des élèves détenteurs d'un CESS du secondaire technique de qualification est de $47 \%$, dont $4,5 \%$ se dirigent vers le supérieur de niveau universitaire et $42,5 \%$ vers le supérieur de type court. Quant au taux d'accès à l'enseignement supérieur, limité au type court, des élèves ayant obtenu un CESS en fin d'enseignement secondaire professionnel, il n'est que de $14 \%$.

1 Hors formes artistique de transition et de qualification, les Écoles Supérieures des Arts n'entrant pas en compte, par manque de données disponibles, dans le calcul du nombre d'entrants dans l'enseignement supérieur.

2 L'enseignement supérieur se compose des Universités, des Hautes Écoles et des Instituts Supérieurs d'Architecture ; les Écoles Supérieures des Arts n'entrent pas en compte par manque de données disponibles pour le calcul des indicateurs.

3 Les individus entrant pour la première fois dans l'enseignement supérieur à l'âge de 23 ans et plus n'ont pas pu être pris en compte dans le calcul des taux d'accès de la figure 1.

4 Les élèves ayant obtenu le CESS en 2003 et entrant pour la première fois dans l'enseignement supérieur en 2003-2004, 2004-2005 et 2005-2006 sont pris en compte dans le calcul des taux d'accès de la figure 2 ; par contre, les élèves qui entrent pour la première fois dans l'enseignement supérieur en 2006-2007 et après n'ont pas pu être comptabilisés. 
$100 \%$

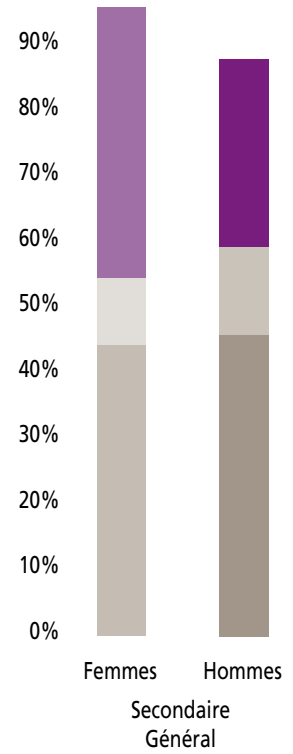

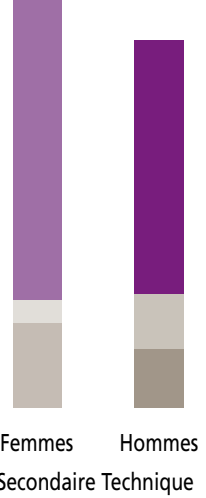
de transition

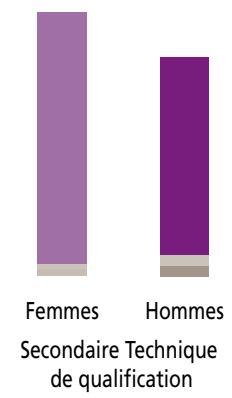

SHU de type court

SHU de type long

Universités

Le taux d'accès à l'enseignement supérieur des femmes nées en 1983 et présentes dans l'enseignement secondaire général en 2000-2001, c'est-à-dire à l'âge des 17 ans, est de 95 , dont $44 \%$ s'orientant vers l'université, $10 \%$ vers

le SHU de type long et $41 \%$ vers le SHU de type court.
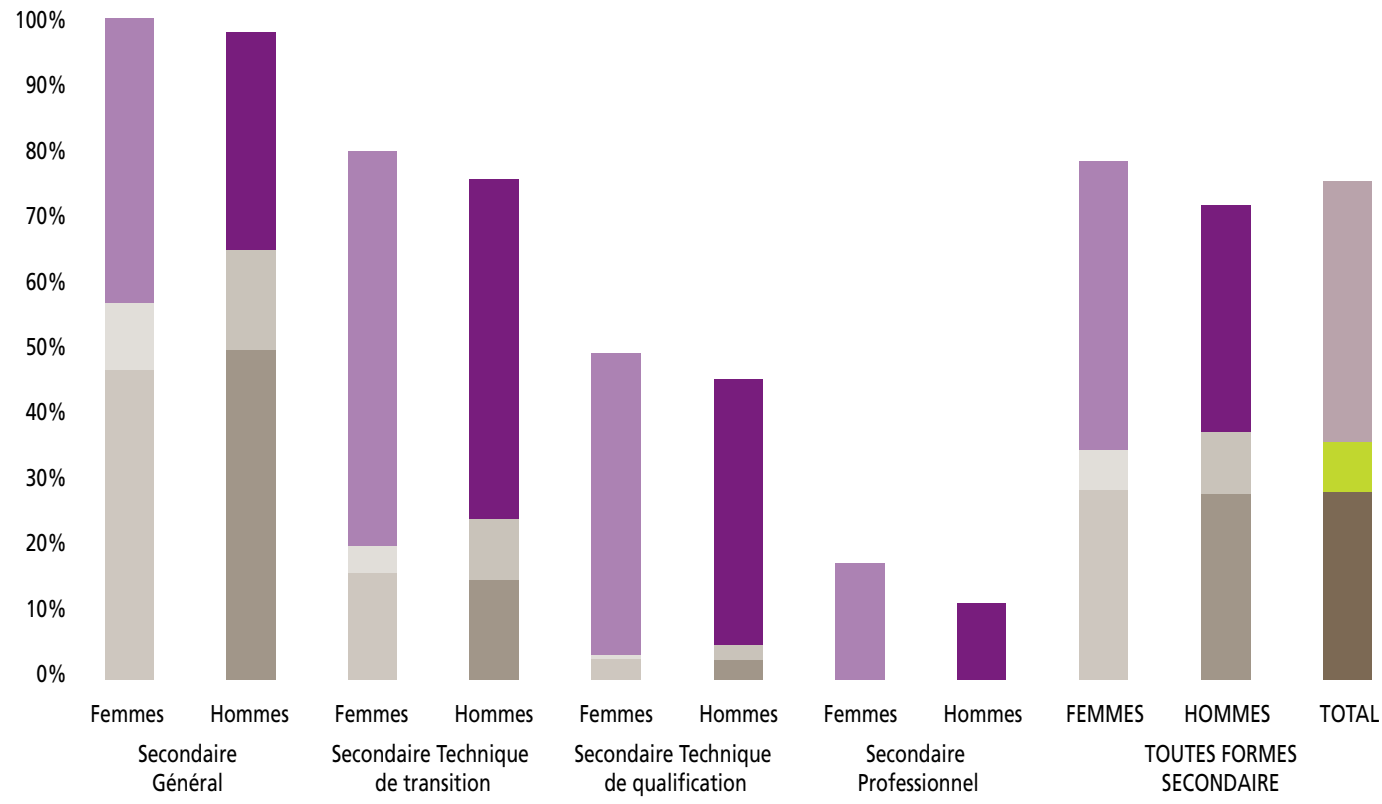

Le taux d'accès à l'enseignement supérieur des femmes ayant obtenu le CESS de l'enseignement secondaire général en fin d'année 2002-2003 est proche des $100 \%$, dont $47 \%$ s'orientant vers l'université, $10 \%$ vers le SHU de type long et $43 \%$ vers le SHU de type court. 
En $2^{\mathrm{e}}$ primaire, $60 \%$ des élèves font preuve de stratégies efficaces de compréhension en lecture.

Les caractéristiques individuelles des élèves influencent peu leurs résultats au test, mais elles jouent déjà sur l'attitude par rapport à la lecture.

Les classes qui accueillent un nombre significatif d'élèves « à risque » ont en moyenne des résultats plus faibles que les autres classes.

es premières évaluations externes ont été organisées par le Service général du Pilotage du système éducatif en 1994. Depuis 2006-2007, ces évaluations sont organisées chaque année en $2^{\mathrm{e}}$ et $5^{\mathrm{e}}$ années primaires et en $2^{\mathrm{e}}$ année secondaire dans une discipline. Ces évaluations non certificatives concernent l'ensemble des établissements d'enseignement en Communauté française. Elles n'ont pas d'incidence sur le parcours des élèves. Elles constituent un outil diagnostique informant les équipes éducatives et les responsables du système sur le niveau d'avancement des élèves. L'exploitation des résultats par les équipes éducatives, soutenues par les services d'inspection et par les services de conseil pédagogique, vise à construire et mettre en œuvre des stratégies pédagogiques et organisationnelles susceptibles d'améliorer les performances des élèves.

II faut attirer l'attention sur le fait que les épreuves de $2^{\mathrm{e}}$, de $5^{\mathrm{e}}$ primaire et de $2^{\mathrm{e}}$ secondaire sont $\mathrm{d}^{\prime}$ une difficulté inégale. Les compétences évaluées, les supports (longueur, difficulté des textes), le mode de questionnement sont en partie différents et aucun procédé technique ne permet de comparer directement les résultats, qu'ils soient exprimés en pourcentage de réussite ou en niveaux de compétences. Dans ces conditions, l'examen des chiffres ne permet pas de conclure à une baisse de niveau de la $2^{\mathrm{e}}$ à la $5^{\mathrm{e}}$ primaire ou à la $2^{\mathrm{e}}$ secondaire.

Les acquis en lecture des élèves de $2^{\mathrm{e}}$ primaire ${ }^{1}$ ont été évalués en février 2007 (Ministère de la Communauté française, 2007)². L'épreuve de $2^{\mathrm{e}}$ primaire comporte à la fois des questions relatives à la compréhension en lecture proprement dite (compréhension de phrases, compréhension d'un texte entier) ainsi que des questions portant sur des compétences plus techniques (localisation de syllabes ou de sons, maîtrise des concepts de lettre, mot, phrase, ...). La figure 1 détaille les scores moyens des élèves aux différentes parties de l'épreuve. Le score moyen à la partie portant sur la compréhension en lecture est de 71,8 \%. Le score moyen à la partie de l'épreuve portant sur la compréhension des concepts métalinguistiques (lettre, mot, phrase, ponctuation) est de 72,1 \%. La partie de l'épreuve consacrée à la reconnaissance des syllabes et des sons est très bien réussie, avec un score moyen de $89,6 \%$.

La partie du test portant sur la compréhension en lecture a été soumise à une analyse spécifique à l'épreuve administrée. Cette analyse a permis d'établir des profils de compétences en lecture et de déterminer la proportion d'élèves de $2^{\mathrm{e}}$ primaire aux différents niveaux. Ainsi, la figure 2 indique qu'une infime partie des élèves (1,3\%) peuvent être qualifiés de " non-lecteurs ", n'ayant pas encore acquis les compétences techniques de base qui permettent d'accéder à la compréhension. Un cinquième des élèves ne dépassent pas le niveau « élémentaire » (niveau 1). À ce niveau, les élèves sont capables de localiser et restituer telle quelle une information donnée dans un texte, d'apparier paroles et locuteur dans un dialogue simple, de comprendre des phrases isolées composées de mots simples, d'effectuer une inférence simple. Au niveau 2, les élèves sont capables de traiter, au sein de phrases isolées, des unités lexicales et grammaticales, de faire un lien entre elles, d'effectuer des inférences simples, parfois en combinant plusieurs informations du texte. Cette description s'applique à un peu plus de quinze pour cent des élèves. Enfin, plus de $60 \%$ des élèves se situent aux niveaux 3 et 4 : au milieu de la $2^{\mathrm{e}}$ primaire, nombreux sont ceux qui peuvent décoder des informations complexes ou implicites, établir des relations anaphoriques (entre un pronom et son référent par exemple). Au niveau 4, les élèves peuvent développer une réponse construite qui atteste de leur compréhension globale, de leur capacité à distinguer le réel de l'imaginaire, de leur capacité à faire des inférences complexes. Ces élèves font déjà preuve, à leur niveau, de stratégies efficaces de compréhension en lecture.

Les comparaisons des scores moyens de catégories d'élèves contrastées (figure 3) indiquent que les différences entre élèves sont peu accusées en fonction de facteurs tels que le sexe, le nombre de livres à la maison, la profession des parents ou le fait de redoubler la $2^{\mathrm{e}}$. Par contre, les élèves qui ne parlent pas toujours le français chez eux ont en moyenne de moins bons résultats en lecture, ce qui indique qu'une attention particulière doit être accordée à cette catégorie d'élèves.

Par contre, pour toutes les catégories d'élèves examinées, on constate des différences plus marquées en termes d'attitudes envers la lecture $^{3}$ (figure 4). Ceci indique que, dès le début des apprentissages, les facteurs motivationnels, qui ont un rôle important dans le maintien et le développement ultérieur des compétences, doivent être pris en compte pour prévenir les décrochages.

Enfin, bien que les caractéristiques des élèves n'affectent pas, en moyenne, leurs résultats au test (figure 3), elles ont un impact au niveau du résultat des classes (figure 5). En effet, les classes qui accueillent un nombre significatif d'élèves potentiellement en difficulté (parce qu'ils vivent dans un milieu peu favorisé, qu'ils ne parlent pas le français chez eux, qu'ils ont déjà redoublé) ont des résultats plus faibles que les autres classes.

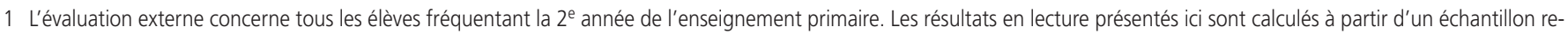
présentatif composé de 3226 élèves de l'enseignement ordinaire répartis dans 238 classes et 117 écoles. Une évaluation des compétences en production écrite a été menée parallèlement à celle des compétences en lecture. Ces résultats ne font pas l'objet de cet indicateur.

2 Les carnets de test, les résultats et les pistes didactiques sont disponibles sur http://www.enseignement.be.

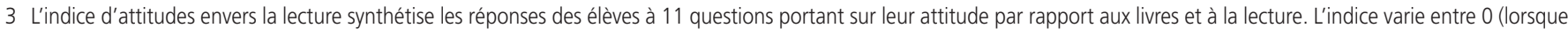
toutes les réponses fournies témoignent d'une attitude négative par rapport à la lecture) et 11 (lorsque toutes les réponses témoignent d'une attitude positive par rapport à la lecture). 
20.1 Score moyen à l'épreuve de lecture, selon le type de compétences mobilisées, $2^{\mathrm{e}}$ primaire - 2007

\begin{tabular}{|c|c|c|}
\hline $\begin{array}{c}\text { Score } \\
\text { « compréhension » }\end{array}$ & $\begin{array}{c}\text { Score } \\
\text { " structuration » }\end{array}$ & $\begin{array}{c}\text { Score } \\
\text { « métalinguistique » }\end{array}$ \\
\hline $\begin{array}{l}\text { Lecture de texte } \\
\text { et phrases } \\
\text { (30 items) }\end{array}$ & $\begin{array}{l}\text { Phonologie et } \\
\text { segmentation } \\
\text { syllabique } \\
\text { (13 items) }\end{array}$ & $\begin{array}{l}\text { Compréhension des } \\
\text { concepts de lettes, } \\
\text { phrases, mots, } \\
\text { ponctuation } \\
\text { (7 items) }\end{array}$ \\
\hline $71,8 \%$ & $89,6 \%$ & $72,1 \%$ \\
\hline
\end{tabular}

Le score moyen en compréhension en lecture est de $71,8 \%$. Le score « structuration » est de $89,6 \%$

20.3 Score moyen à l'ensemble de l'épreuve de lecture, selon les caractéristiques individuelles des élèves, $2^{\mathrm{e}}$ primaire -2007

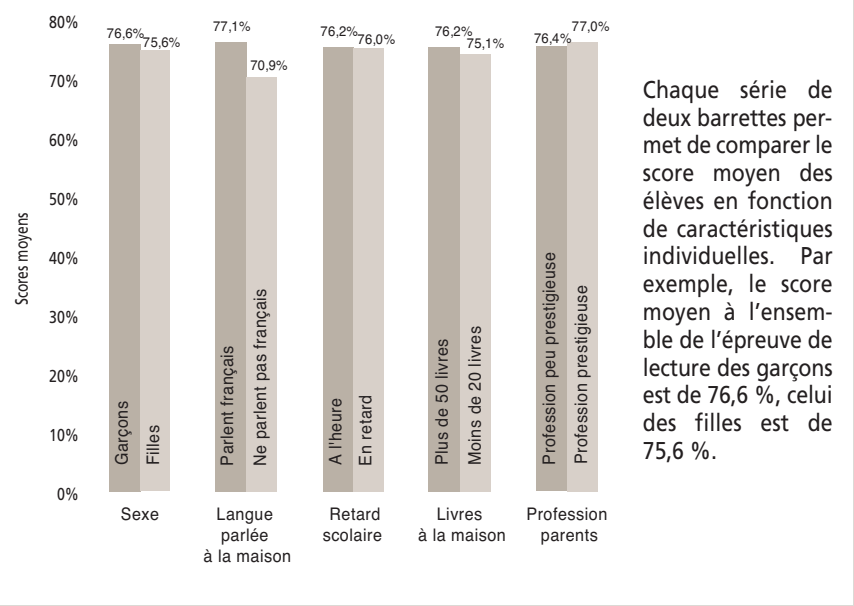

20.2 Répartition des élèves de $2^{\mathrm{e}}$ primaire selon leur niveau de compétences en compréhension en lecture - 2007

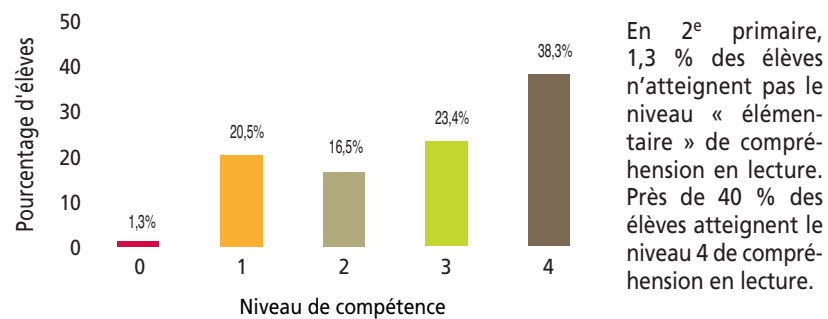

\subsection{Indice d'attitudes envers la lecture, selon les caractéristiques personnelles et scolaires des élèves, $2^{\mathrm{e}}$ primaire -2007}

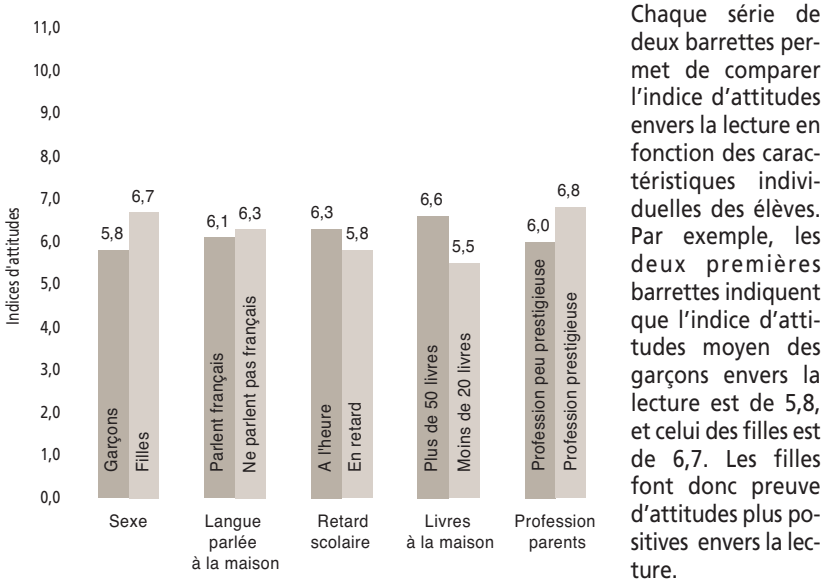

20.5 Score moyen des classes selon les caractéristiques de leurs élèves, $2^{\mathrm{e}}$ primaire - 2007

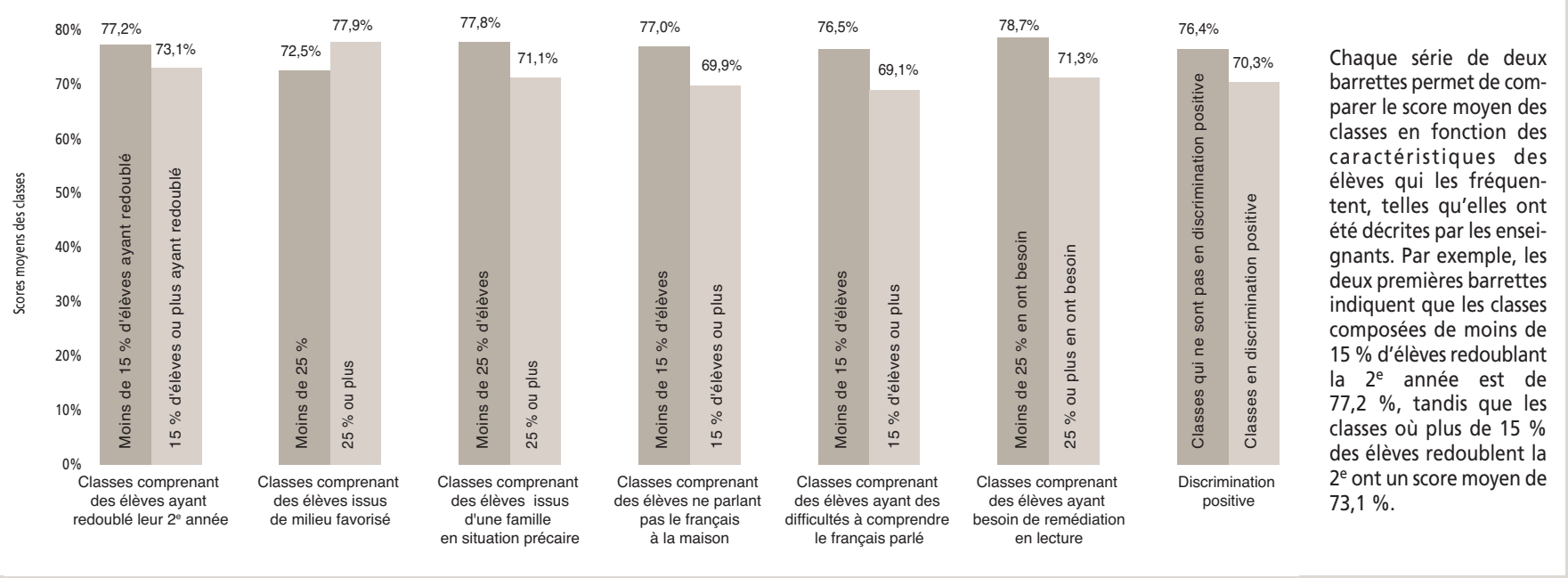


En $5^{\mathrm{e}}$ primaire, un tiers des élèves font preuve d'une bonne maîtrise de la compréhension en lecture. Les autres élèves éprouvent des difficultés à dépasser une analyse très locale des textes. Une minorité d'entre eux sont en grande difficulté.

Tous les facteurs traduisant un contexte socioéconomique ou culturel défavorisé sont associés à une moins bonne maîtrise de la lecture. Ces clivages sociaux se marquent également au niveau des différences entre classes.

es premières évaluations externes ont été organisées par le Service - général du Pilotage du système éducatif en 1994. Depuis 2006-2007, ces évaluations sont organisées chaque année en $2^{\mathrm{e}}$ et $5^{\mathrm{e}}$ années primaires et en $2^{\mathrm{e}}$ année secondaire dans une discipline. Ces évaluations non certificatives concernent l'ensemble des établissements d'enseignement en Communauté française. Elles n'ont pas d'incidence sur le parcours des élèves. Elles constituent un outil diagnostique informant les équipes éducatives et les responsables du système sur le niveau d'avancement des élèves. L'exploitation des résultats par les équipes éducatives, soutenues par les services d'inspection et par les services de conseil pédagogique, vise à construire et mettre en œuvre des stratégies pédagogiques et organisationnelles susceptibles d'améliorer les performances des élèves.

Il faut attirer l'attention sur le fait que les épreuves de $2^{\mathrm{e}}$, de $5^{\mathrm{e}}$ primaire et de $2^{\mathrm{e}}$ secondaire sont d'une difficulté inégale. Les compétences évaluées, les supports (longueur, difficulté des textes), le mode de questionnement sont en partie différents et aucun procédé technique ne permet de comparer directement les résultats, qu'ils soient exprimés en pourcentage de réussite ou en niveaux de compétences. Dans ces conditions, l'examen des chiffres ne permet pas de conclure à une baisse de niveau de la $2^{\mathrm{e}}$ à la $5^{\mathrm{e}}$ primaire ou à la $2^{\mathrm{e}}$ secondaire.

Les acquis en lecture des élèves de $5^{\mathrm{e}}$ primaire ont été évalués en février 2007 (Ministère de la Communauté française, 2007) ${ }^{1}$. L'épreuve ne permet pas d'évaluer l'ensemble des compétences à certifier en fin de $6^{\mathrm{e}}$, mais elle offre un bon diagnostic des performances des élèves dans la plupart des grands domaines de compétences prévus par les Socles de compétences : élaborer des significations, dégager l'organisation d'un texte, percevoir la cohérence entre phrases ainsi que traiter les unités lexicales et grammaticales en contexte.

Le score moyen des élèves est de $67,1 \%$. Ce score moyen sert de point de référence auquel comparer les résultats d'une partie du test ou de certaines catégories d'élèves (figures 1 et 4). Ainsi, la figure 1 montre de nettes différences selon que les questions portent sur des éléments explicites ou implicites, ces dernières étant bien moins réussies. D'autres paramètres ont influencé la difficulté de l'épreuve : la longueur du texte, le texte lui-même et, dans une moindre mesure le type de texte (narratif vs informatif).

Les items du test de lecture ont été soumis à une analyse spécifique à l'épreuve administrée (il n'y a donc pas de comparaison possible avec les résultats des autres années d'études). Cette analyse a permis de dé- terminer la proportion d'élèves aux différents niveaux. Ainsi, la figure 2 indique que plus d'un tiers des élèves de $5^{\mathrm{e}}$ primaire se situent aux niveaux 3 et 4 : ces élèves font preuve d'une bonne, voire d'une très bonne maîtrise, dans différents contextes, de la plupart des compétences évaluées. Ainsi, au niveau 3, les élèves peuvent mettre en relation plusieurs informations explicitement mentionnées dans un texte long, construire une nouvelle information à partir de plusieurs informations explicites, comprendre des doubles sens, et identifier des organisateurs textuels. Au niveau 4, les élèves comprennent de manière approfondie des textes longs, effectuent des inférences complexes en mettant en relation des informations situées à différents endroits du texte, et peuvent interpréter un texte en le mettant en relation avec leurs connaissances personnelles. La moitié des élèves se situent au niveau 2 : ils sont capables de repérer une information dans un texte, d'effectuer une interprétation, de dégager la cohérence d'un extrait de texte, mais à condition que l'analyse reste très " locale ", et ils éprouvent des difficultés pour entrer dans l'implicite des textes qui leur ont été proposés, ce qui indique la direction du travail à poursuivre avec eux en classe. Quant aux $13 \%$ d'élèves situés au niveau 1 , ils réussissent principalement les questions nécessitant de repérer une information courte explicitement mentionnée dans le texte ou de percevoir le lien entre des informations présentées dans une seule phrase pour, par exemple, désigner un personnage ou comprendre le sens d'un mot élémentaire en langue française, ce qui est tout à fait insuffisant à ce niveau d'enseignement.

Lorsque l'on compare les résultats des élèves en fonction de leurs caractéristiques individuelles ou scolaires (figure 3), on constate d'importantes différences pour toutes les caractéristiques envisagées, à l'exception du sexe. Tous les facteurs traduisant un contexte socioéconomique ou culturel moins favorable (profession des parents, nombre de livres, pratiques de lecture des parents, langue parlée à la maison...) sont associés à des résultats moyens en lecture nettement inférieurs à ceux des élèves favorisés. Ces informations interrogent l'École sur sa difficulté à compenser les handicaps socioéconomiques, et ce, dès l'enseignement primaire.

La figure 4 apporte un premier élément de réponse à un problème qui semble structurel. En effet, on sait qu'en Communauté française, les différences entre écoles sont très importantes, notamment en raison de clivages sociaux marqués. La figure 4 met en évidence ce type de phénomènes, en montrant clairement les différences entre classes en fonction de leur composition sociale.

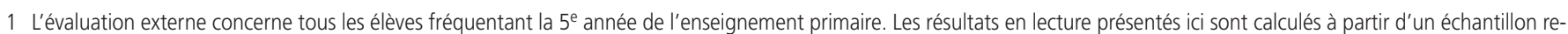
présentatif composé de 3262 élèves fréquentant la $5^{\mathrm{e}}$ primaire ordinaire, répartis dans 226 classes et 117 écoles. Une évaluation des compétences en production écrite a été menée parallèlement à celle des compétences en lecture. Ces résultats ne font pas l'objet de cet indicateur.

2 Les carnets de test, les résultats et les pistes didactiques sont disponibles sur http://www.enseignement.be. 


\subsection{Score moyen à l'ensemble de l'épreuve de lecture, et selon le type de compétences mobilisées et le type de textes, $5^{\mathrm{e}}$ primaire - 2007}

\begin{tabular}{|c|c|c|c|c|c|c|c|c|}
\hline $\begin{array}{l}\text { Score moyen à } \\
\text { l'ensemble de } \\
\text { l'épreuve de } \\
\text { lecture }\end{array}$ & $\begin{array}{c}\text { Score } \\
\text { "dégager des } \\
\text { informations } \\
\text { explicites » }\end{array}$ & $\begin{array}{c}\text { Score } \\
\text { " dégager des } \\
\text { informations } \\
\text { implicites " }\end{array}$ & $\begin{array}{c}\text { Score } \\
\text { " dégager } \\
\text { l'organisation } \\
\text { d'un texte " }\end{array}$ & $\begin{array}{l}\text { Score « traiter } \\
\text { les unités } \\
\text { grammaticales } \\
\text { et lexicales en } \\
\text { contexte» }\end{array}$ & $\begin{array}{c}\text { Score } \\
\text { " textes courts }\end{array}$ & $\begin{array}{c}\text { Score } \\
\text { « textes longs » }\end{array}$ & $\begin{array}{c}\text { Score } \\
\text { " textes } \\
\text { narratifs » }\end{array}$ & $\begin{array}{c}\text { Score } \\
\text { "textes } \\
\text { informatifs " }\end{array}$ \\
\hline (80 items) & (26 items) & (17 items) & (15 items) & (16 items) & (39 items) & (41 items) & (47 items) & (33 items) \\
\hline $67,1 \%$ & $75,8 \%$ & $65,4 \%$ & $67,3 \%$ & $62,5 \%$ & $71,1 \%$ & $63,5 \%$ & $68,6 \%$ & $64,9 \%$ \\
\hline
\end{tabular}

Le score moyen à l'épreuve de lecture est de $67,1 \%$. Le sous-score spécifique en traitement de l'information explicite est de $75,8 \%$, il est supérieur au score moyen, tandis que le sous-score en traitement de l'information implicite $(65,4 \%)$ est inférieur au score moyen.

\subsection{Répartition des élèves de $5^{\mathrm{e}}$ primaire selon leur niveau de compétences compréhension en lecture - 2007}

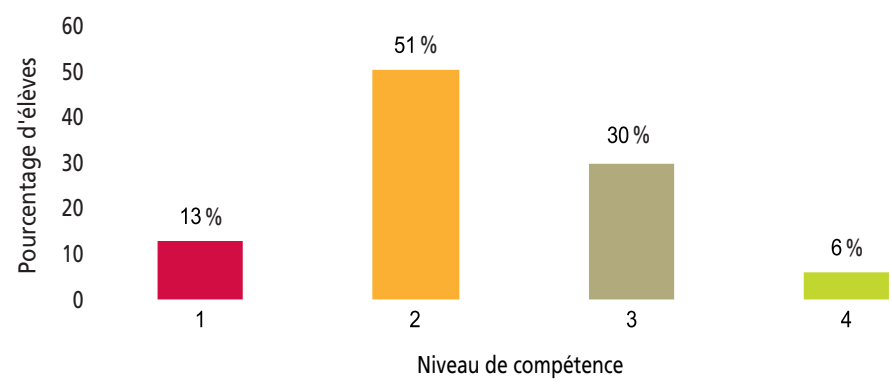

En $5^{e}$ primaire, $13 \%$ des élèves ont un niveau de lecture rudimentaire de compréhension en lecture. Trente pour cent des élèves atteignent le niveau 3 : les compétences devant être maîtrisées en fin de $6^{\mathrm{e}}$ primaire sont en très bonne voie d'acquisition.

Niveau de compétence

21.3 Scores moyens en lecture, selon les caractéristiques individuelles et scolaires des élèves, $5^{e}$ primaire - 2007

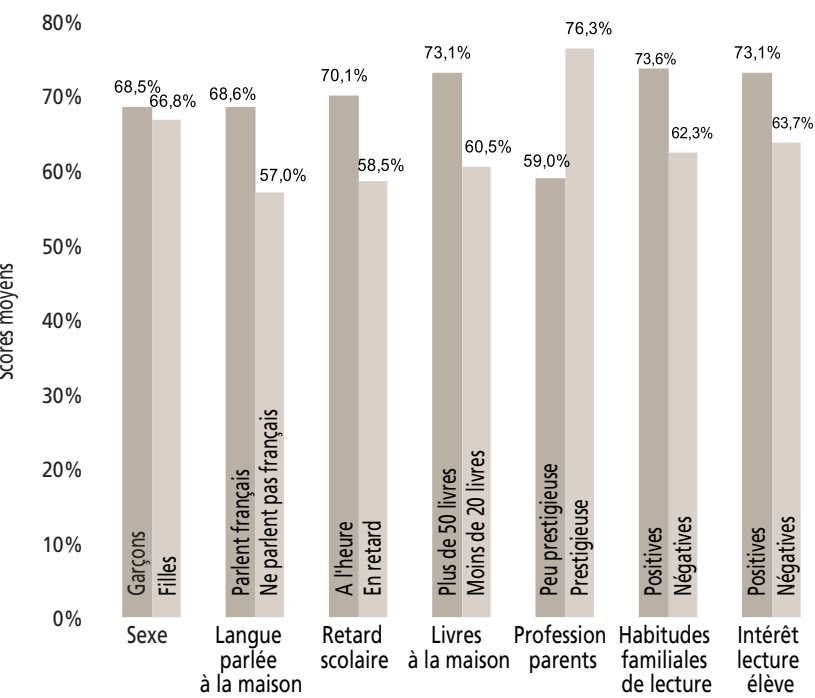

Chaque série de deux barrettes présente les scores moyens de catégories d'élèves contrastées. La $2^{\mathrm{e}}$ série de barrettes indique que les élèves qui ne parlent pas le français chez eux ont un score moyen de $57 \%$. Ceux qui disent toujours parler le français chez eux ont un score moyen de $68,6 \%$.
21.4 Scores moyens en lecture des classes, selon leur composition, $5^{e}$ primaire - 2007

$80 \%$

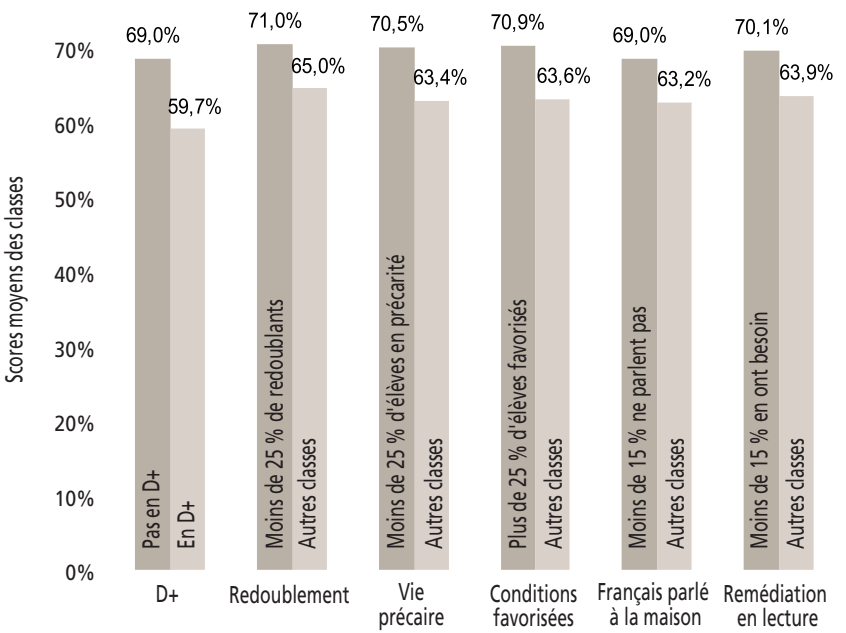

Chaque série de deux barrettes permet de comparer le score moyen des classes en fonction de leurs caractéristiques, telles qu'elles ont été décrites par les enseignants. Ainsi, les deux premières barrettes montrent que le score moyen d'une classe en discrimination positive est de $59,7 \%$, et celui d'une classe non en discrimination positive est de $69 \%$. 


\section{Compétences en lecture des élèves de $2^{e}$ secondaire - 2007}

En $2^{\mathrm{e}}$ secondaire commune et complémentaire, la majorité des élèves ont acquis des compétences de base en lecture, mais ont encore des difficultés à interagir personnellement avec un texte ou à faire des inférences complexes.

La majorité des élèves de $2^{\mathrm{e}}$ secondaire professionnelle sont en grande difficulté de lecture.

Les clivages sociaux se marquent au niveau des résultats en lecture, tant au niveau individuel qu'au niveau des classes qui accueillent un nombre significatif d'élèves « à risque ».

es premières évaluations externes ont été organisées par le Service général du Pilotage du système éducatif en 1994. Depuis 2006-2007, ces évaluations sont organisées chaque année en $2^{\mathrm{e}}$ et $5^{\mathrm{e}}$ années primaires et en $2^{\mathrm{e}}$ année secondaire dans une discipline. Ces évaluations non certificatives concernent l'ensemble des établissements d'enseignement en Communauté française. Elles n'ont pas d'incidence sur le parcours des élèves. Elles constituent un outil diagnostique informant les équipes éducatives et les responsables du système sur le niveau d'avancement des élèves. L'exploitation des résultats par les équipes éducatives, soutenues par les services d'inspection et par les services de conseil pédagogique, vise à construire et mettre en œuvre des stratégies pédagogiques et organisationnelles susceptibles d'améliorer les performances des élèves.

Il faut attirer l'attention sur le fait que les épreuves de $2^{\mathrm{e}}$, de $5^{\mathrm{e}}$ primaire et de $2^{\mathrm{e}}$ secondaire sont d'une difficulté inégale. Les compétences évaluées, les supports (longueur, difficulté des textes), le mode de questionnement sont en partie différents et aucun procédé technique ne permet de comparer directement les résultats, qu'ils soient exprimés en pourcentage de réussite ou en niveaux de compétences. Dans ces conditions, l'examen des chiffres ne permet pas de conclure à une baisse de niveau de la $2^{\mathrm{e}}$ à la $5^{\mathrm{e}}$ primaire ou à la $2^{\mathrm{e}}$ secondaire.

Les acquis en lecture des élèves de $2^{\mathrm{e}}$ secondaire ${ }^{1}$ ont été évalués en février 2007 (Ministère de la Communauté française, 2007)². L'évaluation de $2^{\mathrm{e}}$ secondaire est centrée sur les compétences liées à l'élaboration de significations. Deux épreuves comportant une partie commune ont été conçues. Les compétences évaluées sont identiques pour les élèves de $2^{\mathrm{e}}$ professionnelle $\left(2^{\mathrm{e}} \mathrm{P}\right)$, de $2^{\mathrm{e}}$ commune et de $2^{\mathrm{e}}$ complémentaire $\left(2^{\mathrm{e}}\right.$ C) : elles portent sur la compréhension globale et locale d'un texte, la compréhension du vocabulaire, l'organisation du texte et la maîtrise des anaphores. Cependant, l'épreuve mise au point pour les élèves de $2^{\mathrm{e}}$ commune et de $2^{\mathrm{e}}$ complémentaire $\left(2^{\mathrm{e}} \mathrm{C}\right)$ est plus longue et plus complexe que l'épreuve proposée aux $2^{\mathrm{e}} \mathrm{P}$.

Le score moyen des élèves de $2^{e} \mathrm{C}$ est de $58,6 \%$. En $2^{\mathrm{e}} \mathrm{P}$, les élèves ont un score moyen de $48,1 \%$.

Les items du test de lecture ont été soumis à une analyse spécifique à l'épreuve administrée (il n'y a donc pas de comparaison possible avec les résultats des autres années d'études, ni entre les élèves de $2^{\mathrm{e}} \mathrm{C}$ et de $\left.2^{\mathrm{e}} \mathrm{P}\right)$. Cette analyse a permis d'établir des profils de compétence en lecture et de déterminer la proportion d'élèves aux différents niveaux de compétences.
La figure 1 indique que la majorité des élèves de $2^{\mathrm{e}} \mathrm{C}$ se situent au niveau 2 de compétences. À ce niveau, les élèves sont capables de combiner plusieurs informations provenant d'un texte long, d'effectuer des inférences simples et de dégager le sens global d'un texte. Ils éprouvent cependant des difficultés lorsqu'il s'agit d'interagir personnellement avec un texte ou d'effectuer des inférences plus complexes. Un cinquième des élèves de $2^{\mathrm{e}} \mathrm{C}$ font preuve d'une maîtrise plus avancée, puisqu'ils peuvent faire des inférences à partir d'informations implicites dispersées dans un texte, proposer une interprétation personnelle en se fondant sur un texte, et dégager le sens global d'un récit long et « résistant » (niveau 3). Par contre, près d'un élève sur cinq éprouve des difficultés à dépasser le traitement local des informations (localisation et restitution d'une information donnée dans un texte) (niveau 1).

La figure 2 montre que la majorité des élèves de $2^{\mathrm{e}} \mathrm{P}$ sont en difficulté, dans la mesure où ils appliquent essentiellement des stratégies de repérage de mots et effectuent des inférences très simples (niveau 1). Près d'un quart des élèves de $2^{\mathrm{e}} \mathrm{P}$ se situent au niveau 2 . À ce niveau, les élèves sont capables de localiser une information courte explicitement mentionnée dans le texte, sans que les termes utilisés dans la question correspondent à ceux utilisés dans le texte, et d'effectuer des inférences simples. Enfin, $17 \%$ d'entre eux se situent aux niveaux 3 ou 4, où il s'agit de combiner plusieurs informations, de réaliser des interprétations plus complexes ou encore de dégager le sens global d'un récit.

Lorsque l'on compare les résultats des élèves à l'ensemble du test de lecture en fonction de leurs caractéristiques individuelles ou scolaires (figures 3 et 4), on constate que les différences entres les filles et les garçons sont peu importantes, mais que les autres caractéristiques des élèves, comme leur statut par rapport à l'immigration, les pratiques de lecture au sein des familles, les pratiques et attitudes personnelles des élèves par rapport à la lecture ou l'année d'études fréquentée un an avant l'évaluation, sont autant d'éléments liés à des différences de résultats.

Les figures 5 et 6 présentent les moyennes des classes en fonction des caractéristiques des élèves qui les composent, telles qu'elles ont été décrites par les enseignants. Que ce soit en $2^{\mathrm{e}}$ commune ou en $2^{\mathrm{e}}$ professionnelle, les classes qui regroupent des élèves moins favorisés ont presque systématiquement des résultats inférieurs aux autres classes. Les moyens accordés dans le cadre des mesures de discrimination positive ne permettent pas de compenser suffisamment le handicap dû à la concentration d'élèves moins favorisés.

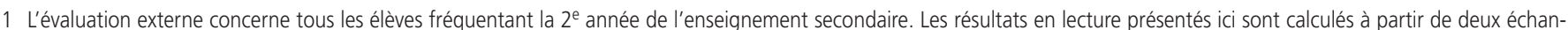
tillons représentatifs composés de 1850 élèves répartis dans 100 classes et 100 écoles pour ce qui est des $2^{\mathrm{e}}$ communes et complémentaires, et de 1093 élèves répartis dans 100 classes et 100 écoles en ce qui concerne les élèves de $2^{e}$ professionnelle. Une évaluation des compétences en production écrite a été menée parallèlement à celle des compétences en lecture. Ces résultats ne font pas l'objet de cet indicateur.

2 Les carnets de test, les résultats et les pistes didactiques sont disponibles sur http://www.enseignement.be. 
22.1 Répartition des élèves de $2^{\mathrm{e}} \mathrm{C}$ selon leur niveau de compétences compréhension en lecture $-\mathbf{2 0 0 7}$

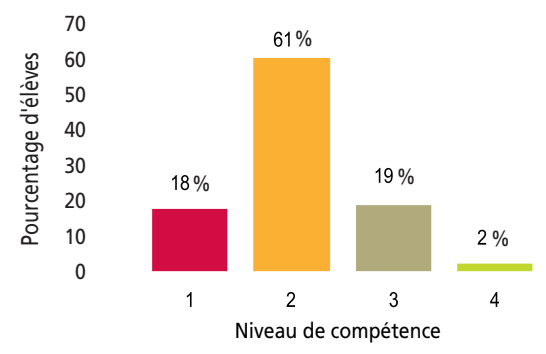

En $2^{\mathrm{e}}$ commune et complémentaire $\left(2^{\mathrm{e}} \mathrm{C}\right), 18 \%$ des élèves se situent au niveau " élémentaire » de compréhension en lecture. Dix-neuf pour cent des élèves de $2^{\mathrm{e}} \mathrm{C}$ atteignent le niveau 3 de compréhension en lecture.
22.3 Scores moyens en lecture, selon les caractéristiques personnelles et scolaires des élèves, $2^{\mathrm{e}} \mathrm{C}-2007$

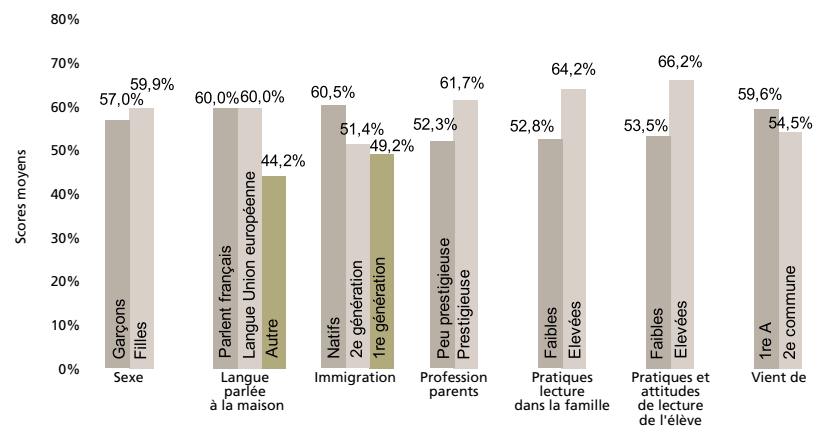

Chaque série de deux barrettes permet de comparer le score moyen des élèves en fonction de caractéristiques individuelles. Par exemple, le score moyen à l'ensemble de l'épreuve de lecture des garçons de $2^{\mathrm{e}}$ commune et complémentaire $\left(2^{\mathrm{e}} \mathrm{C}\right.$ ) est de $57,0 \%$, celui des filles est de $59,9 \%$.

\subsection{Scores moyens des classes selon les caractéristiques de leurs élèves,} $2^{\mathrm{e}}$ secondaire C - 2007

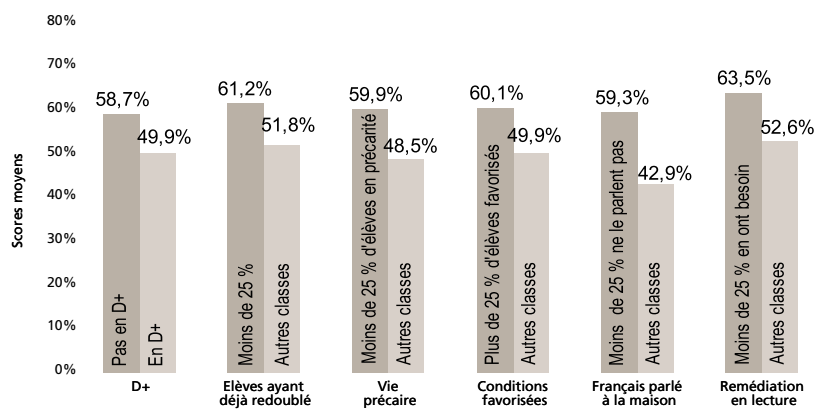

Chaque série de deux barrettes permet de comparer le score moyen des classes en fonction de leur composition, telle qu'elle a été décrite par les enseignants. Ainsi, les deux premières barrettes montrent que le score moyen d'une classe de $2^{\mathrm{e}}$ commune ou complémentaire en discrimination positive est de $49,9 \%$, et celui d'une classe non en discrimination positive est de $58,7 \%$.
22.2 Répartition des élèves de $2^{\mathrm{e}} \mathrm{P}$ selon leur niveau de compétences compréhension en lecture - 2007

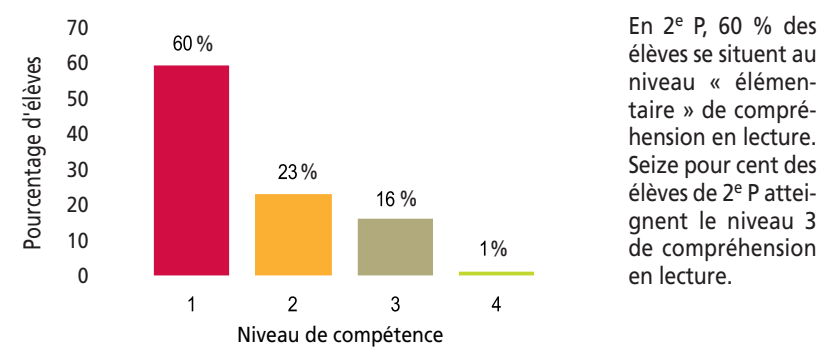

\subsection{Scores moyens en lecture, selon les caractéristiques personnelles et scolaires des élèves, $2^{e}$ P -2007}

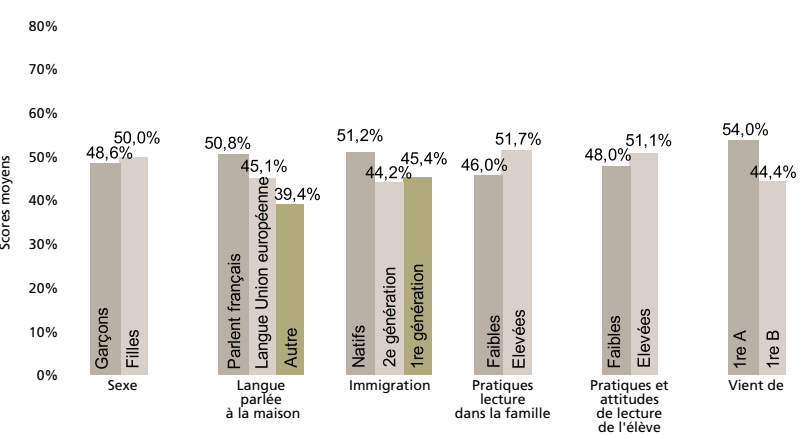

Chaque série de deux barrettes permet de comparer le score moyen des élèves en fonction de caractéristiques individuelles. Par exemple, le score moyen à l'ensemble de l'épreuve de lecture des garçons de $2^{\mathrm{e}} \mathrm{P}$ est de $48,6 \%$, celui des filles est de $50,0 \%$. La profession des parents n'a pas été retenue pour les $2^{\mathrm{e}} \mathrm{P}$ : la majorité des parents d'élèves de $2^{\mathrm{e}} \mathrm{P}$ ont des statuts socioprofessionnels peu élevés. La catégorie « professions prestigieuses » compte trop peu de sujets pour être pertinente.

\subsection{Scores moyens des classes selon les caractéristiques de leurs élèves,} $2^{\mathrm{e}}$ secondaire $\mathrm{P}-2007$

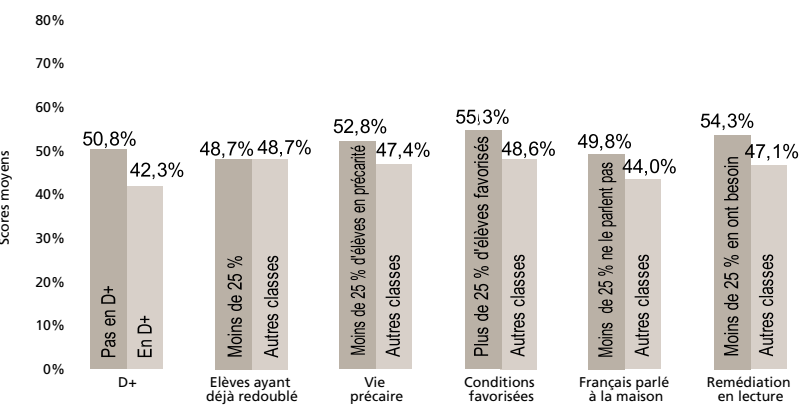

Chaque série de deux barrettes permet de comparer le score moyen des classes en fonction de leur composition, telle qu'elle a été décrite par les enseignants. Ainsi, les deux premières barrettes montrent que le score moyen d'une classe de $2^{\mathrm{e}} \mathrm{P}$ en discrimination positive est de $42,3 \%$, et celui d'une classe non en discrimination positive est de $50,8 \%$ 


\section{Compétences en sciences des élèves de 15 ans - 2006}

À 15 ans, les élèves de la Communauté française savent faire la part des choses entre ce qui relève de la science et ce qui n'en relève pas, reconnaître une démarche scientifique et interpréter des données fournies. En revanche, ils ont en moyenne de faibles résultats lorsque intervient la mobilisation de connaissances scientifiques.

Les différences de performances entres écoles sont importantes. Ces différences s'expliquent en grande partie par des facteurs socioéconomiques.

es pays de l'OCDÉ ont mis en place le Programme International pour le Suivi des Acquis des élèves (PISA). L'objectif de PISA est d'évaluer les performances en lecture, mathématique et sciences des jeunes de 15 ans, où qu'ils soient dans le cursus scolaire. En effet, dans de nombreux systèmes éducatifs, les élèves de 15 ans arrivent en fin d'obligation scolaire et les pays se préoccupent du niveau de compétences des futurs adultes. C'est pourquoi la définition des compétences évaluées résulte d'un consensus international sur les outils à acquérir pour participer activement à la vie civile et professionnelle.

Après avoir mis l'accent sur la lecture en 2000 et les mathématiques en 2003, en 2006, l'évaluation a porté principalement sur la culture scientifique. Le test vise à cerner les connaissances scientifiques et l'usage que I'on en fait pour identifier des questions d'ordre scientifique, acquérir de nouvelles connaissances, expliquer des phénomènes de manière scientifique et tirer des conclusions fondées sur des faits; la compréhension des éléments caractéristiques de la science en tant que forme de connaissance et de compréhension de l'humain ; la conscience du rôle de la science et de la technologie dans la modification de notre environnement matériel, intellectuel et culturel et la volonté de s'engager dans des problématiques scientifiques en tant que citoyen réfléchi.

Le score moyen de la Communauté française ${ }^{1}$ en sciences est de 486, ce qui est inférieur à la moyenne de l'ensemble des pays de l'OCDÉ, égale à 500 , ainsi qu'à la moyenne des autres Communautés belges ou de pays limitrophes tels que l'Allemagne et les Pays-Bas. Par contre, ce score n'est pas statistiquement différent de celui de pays européens comme le Danemark ou la France. L'écart type de la Communauté française, qui mesure la dispersion des scores autour de la moyenne, est quant à lui plus élevé qu'en moyenne dans les pays industrialisés (figure 1).

L'examen de résultats de deux sous-échelles de sciences permet d'affiner ce premier constat (figure 1). Le score moyen de la Communauté française pour la sous-échelle "Connaissances à propos des sciences" est comparable à la moyenne internationale. Cette sous-échelle évalue la capacité à reconnaître les questions qui peuvent faire l'objet d'une investigation scientifique par opposition à des questions d'ordre éthique ou économique par exemple, ou encore la capacité à analyser des résultats scientifiques donnés, ainsi que l'identification des méthodes d'investigation scientifique. Le score de la Communauté française pour la sous-échelle "Expliquer des phénomènes de manière scientifique ", qui évalue l'utilisation des connaissances scientifiques dans des situations proches de la vie réelle, est plus préoccupant, puisqu'il est nettement inférieur à la moyenne internationale. En d'autres termes, les élèves de la
Communauté française ont en moyenne de faibles résultats pour les questions impliquant la mobilisation de connaissances scientifiques, et des résultats satisfaisants pour les questions portant sur les connaissances à propos des sciences.

La figure 2 illustre la proportion d'élèves à chacun des 6 niveaux de compétence sur l'échelle globale de sciences. Le niveau 2 constitue un niveau charnière pour l'OCDÉ, car sous ce niveau, les élèves ont des compétences très limitées qu'ils peuvent seulement utiliser dans quelques situations familières. La Communauté française se caractérise par un nombre important - $24 \%$ - d'élèves sous ce niveau (la moyenne internationale est de $19 \%$ ). Ce sont les élèves qui ont redoublé et/ou qui sont dans des formes qualifiantes qui se situent majoritairement aux niveaux les plus bas de l'échelle de compétences en sciences. En revanche, sept pour cent des élèves de la Communauté française se situent aux niveaux 5 et 6 de l'échelle (pour $9 \%$ au niveau international). Ces élèves peuvent reconnaître les composantes scientifiques dans des contextes complexes, faire preuve d'esprit critique et utiliser leurs connaissances en sciences pour résoudre des problèmes. Environ $15 \%$ des élèves à l'heure de l'enseignement de transition se situent à ces niveaux.

La figure 3 montre les relations entres les caractéristiques des élèves et leurs performances en sciences. Si la différence de résultats en fonction du sexe n'est pas significative, les caractéristiques sociodémographiques telles que le milieu social et le statut par rapport à l'immigration influent fortement sur les résultats. La différence de performance en fonction du niveau socioéconomique est l'une des plus importantes des pays industrialisés. En Communauté française, des variables comme l'image que l'on s'est forgée de ses compétences en sciences et la conscience des problématiques environnementales sont également liées aux résultats en sciences.

Les évaluations internationales permettent de pointer les particularités de notre système éducatif en le comparant à d'autres systèmes. Deux caractéristiques récurrentes de la Communauté française sont illustrées par la figure 4. D'une part, les différences entre écoles sont plus accusées que dans la plupart des pays, puisque près de la moitié des différences de résultats en sciences sont expliquées par l'établissement fréquenté. La Communauté française partage cette caractéristique avec certains pays voisins qui ont également fait le choix d'une différenciation précoce via les mécanismes de filières, d'options et de redoublement. D'autre part, une proportion importante de ces différences entre écoles s'explique par des facteurs socioéconomiques, les écoles ayant tendance à concentrer des élèves provenant de milieux sociaux identiques. 
23.1 Performances moyennes des élèves de 15 ans en culture scientifique et sur deux sous-échelles et dispersion des résultats dans une sélection de pays* -2006

\begin{tabular}{|c|c|c|c|c|c|c|c|c|c|c|}
\hline \multicolumn{3}{|c|}{$\begin{array}{l}\text { Échelle combinée } \\
\text { de culture scientifique }\end{array}$} & \multicolumn{2}{|c|}{ Écarts types } & \multicolumn{3}{|c|}{$\begin{array}{c}\text { Sous-échelle " Expliquer des phénomènes } \\
\text { de manière scientifique » }\end{array}$} & \multicolumn{3}{|c|}{$\begin{array}{l}\text { Sous-échelle "Connaissances } \\
\text { à propos des sciences » }\end{array}$} \\
\hline Finlande & 563 & $(2,0) * *$ & 86 & $(1,0)^{* *}$ & Finlande & 566 & $(2,0) * *$ & Finlande & 558 & $(1,7)^{* *}$ \\
\hline C. flamande & 529 & $(3,2)$ & 93 & $(2,8)$ & C. flamande & 525 & $(3,3)$ & C. flamande & 535 & $(2,9)$ \\
\hline Pays-Bas & 525 & $(2,7)$ & 96 & $(1,6)$ & Pays-Bas & 522 & $(2,7)$ & Pays-Bas & 530 & $(2,6)$ \\
\hline C. germanophone & 516 & $(2,9)$ & 97 & $(2,5)$ & Allemagne & 519 & $(3,7)$ & C. germanophone & 523 & $(3,7)$ \\
\hline Allemagne & 516 & $(3,8)$ & 100 & $(2,0)$ & Royaume-Uni & 517 & $(2,3)$ & Royaume-Uni & 517 & $(1,9)$ \\
\hline Royaume-Uni & 515 & $(2,3)$ & 107 & $(1,5)$ & Autriche & 516 & $(4,0)$ & Irlande & 513 & $(2,7)$ \\
\hline Autriche & 511 & $(3,9)$ & 98 & $(2,4)$ & C. germanophone & 515 & $(2,9)$ & Allemagne & 512 & $(3,1)$ \\
\hline Irlande & 508 & $(3,2)$ & 94 & $(1,5)$ & Suède & 510 & $(2,9)$ & France & 507 & $(3,1)$ \\
\hline Suède & 503 & $(2,4)$ & 94 & $(1,4)$ & Irlande & 505 & $(3,2)$ & Autriche & 504 & $(3,3)$ \\
\hline OCDE & 500 & $(0,5)$ & 95 & $(0,3)$ & Danemark & 501 & $(3,3)$ & OCDE & 500 & $(0,5)$ \\
\hline Danemark & 496 & $(3,1)$ & 93 & $(1,4)$ & OCDE & 500 & $(0,5)$ & Suède & 498 & $(2,2)$ \\
\hline France & 495 & $(3,4)$ & 102 & $(2,1)$ & Espagne & 490 & $(2,4)$ & C. française & 497 & $(3,9)$ \\
\hline Espagne & 488 & $(2,6)$ & 91 & $(1,0)$ & Luxembourg & 483 & $(1,1)$ & Danemark & 493 & $(2,6)$ \\
\hline Luxembourg & 486 & $(1,1)$ & 97 & $(0,9)$ & France & 481 & $(3,2)$ & Espagne & 489 & $(2,0)$ \\
\hline C. française & 486 & $(4,3)$ & 103 & $(3,0)$ & Italie & 480 & $(2,0)$ & Luxembourg & 488 & $(1,3)$ \\
\hline Italie & 475 & $(2,0)$ & 96 & $(1,3)$ & Grèce & 476 & $(3,0)$ & Portugal & 481 & $(2,7)$ \\
\hline Portugal & 474 & $(3,0)$ & 89 & $(1,7)$ & C. française & 473 & $(4,3)$ & Italie & 472 & $(1,8)$ \\
\hline Grèce & 473 & $(3,2)$ & 92 & $(2,0)$ & Portugal & 469 & $(2,9)$ & Grèce & 471 & $(2,8)$ \\
\hline Mexique & 410 & $(2,7)$ & 81 & $(1,5)$ & Mexique & 406 & $(2,7)$ & Mexique & 413 & $(2,1)$ \\
\hline
\end{tabular}

En vert, les pays dont la performance moyenne est supérieure à celle de la Communauté française ; en rouge, ceux dont la performance moyenne est inférieure à la CF : en noir ceux dont la performa nce performance moyenne ne diffère pas significativement de la CF. Le degré de certitude est de $95 \%$.

Les pays sont classés par ordre décroissant, en fonction de leurs performances moyennes.

* La sélection reprend les 3 Communautés de Belgique, des pays européens, et deux pays aux résultats particulièrement élevés ou faibles.

** Il s'agit de l'erreur de mesure. Elle permet de dire si des différences de résultats sont significatives ou non : ainsi, les scores moyens « bruts » de la CF et du Luxembourg sont différents, mais les marges d'erreurs associées à ces scores ne permettent pas de conclure à de réelles différences entre les deux pays.

23.2 Répartition des élèves de 15 ans selon leur niveau de compétences en sciences, en fonction de la section et de l'année d'études, Communauté française - 2006

\begin{tabular}{|c|c|c|c|c|c|c|c|}
\hline & \multirow{2}{*}{$\begin{array}{l}\text { Toutes années } \\
\text { et formes confondues }\end{array}$} & \multirow{2}{*}{$\begin{array}{c}1^{\text {er }} \text { degré } \\
7 \% \text { des élèves de } 15 \text { ans }\end{array}$} & \multicolumn{2}{|c|}{ Qualification } & \multicolumn{2}{|c|}{ Transition } & \multirow{9}{*}{$\begin{array}{l}\text { Dix-sept pour cent } \\
\text { des élèves de } 15 \text { ans } \\
\text { en } 3^{e} \text { année de l'en- } \\
\text { seignement de quali- } \\
\text { fication sont sous le } \\
\text { niveau } 1 \text { de compé- } \\
\text { tences en sciences. } \\
\text { En } 3^{e} \text { année de l'en- } \\
\text { seignement de tran- } \\
\text { sition, seulement } \\
2 \% \text { des élèves se si- } \\
\text { tuent à ce niveau. }\end{array}$} \\
\hline & & & $\begin{array}{c}3^{\text {e }} \text { année } \\
21 \% \text { des élèves de } 15 \text { ans }\end{array}$ & $\begin{array}{c}4^{e} \text { année } \\
12 \% \text { des élèves de } 15 \text { ans }\end{array}$ & $\begin{array}{c}3^{e} \text { année } \\
16 \% \text { des élèves de } 15 \text { ans }\end{array}$ & $\begin{array}{c}4 \text { e année } \\
39 \% \text { des élèves de } 15 \text { ans }\end{array}$ & \\
\hline Niveau 6 & $1 \%(0,2)$ & - & - & - & - & $1 \%(0,5)$ & \\
\hline Niveau 5 & $6 \%(0,8)$ & - & - & - & $2 \%(0,9)$ & $14 \%(1,9)$ & \\
\hline Niveau 4 & $19 \%(1,0)$ & $1 \%(0,7)$ & $2 \%(0,7)$ & $9 \%(1,8)$ & $16 \%(2,2)$ & $36 \%(2,0)$ & \\
\hline Niveau 3 & $26 \%(1,5)$ & $7 \%(2,4)$ & $14 \%(2,3)$ & $28 \%(3,7)$ & $36 \%(3,7)$ & $33 \%(1,6)$ & \\
\hline Niveau 2 & $24 \%(1,6)$ & $22 \%(4,7)$ & $35 \%(3,3)$ & $33 \%(4,4)$ & $31 \%(2,6)$ & $13 \%(1,5)$ & \\
\hline Niveau 1 & $16 \%(1,2)$ & $35 \%(4,1)$ & $33 \%(3,7)$ & $23 \%(3,5)$ & $13 \%(2,1)$ & $2 \%(0,7)$ & \\
\hline Inférieur niv. 1 & $8 \%(1,2)$ & $35 \%(5,1)$ & $17 \%(2,6)$ & $6 \%(1,6)$ & $2 \%(0,8)$ & - & \\
\hline
\end{tabular}

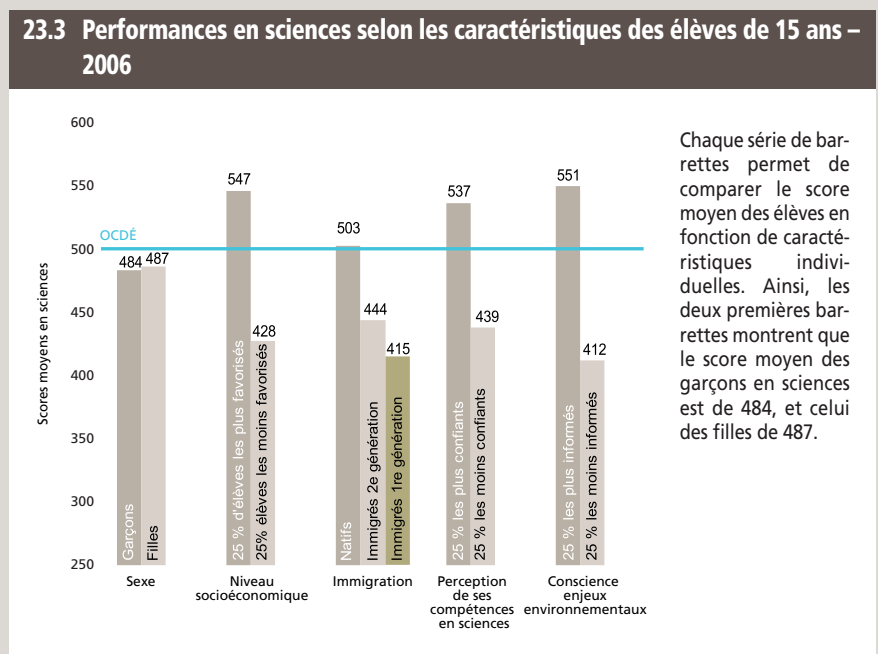

\subsection{Différences de performances en sciences entre écoles - 2006}

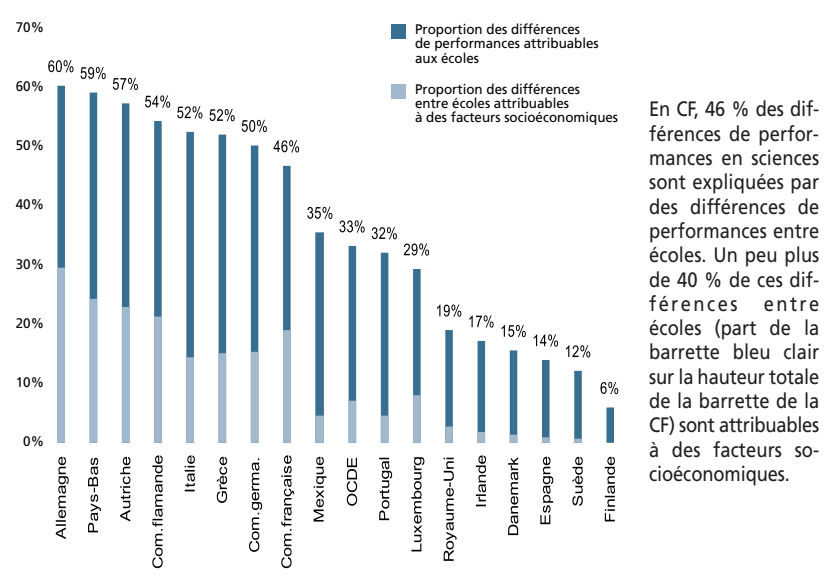


En 2005, 45512 élèves, soit un peu plus de $93 \%$ de l'effectif de $6^{e}$ année de l'enseignement primaire ordinaire, ont obtenu le Certificat d'Études de Base (CEB). Sur les sept dernières années, ce taux varie peu et est en moyenne de $93,3 \%$.

Sur les 52720 élèves inscrits en $5^{\circ}$ primaire en 2002-2003, 90,4 \% obtiennent le CEB en 2 ans, pour 95,4\% en 3 ans et 2443 élèves quittent le primaire sans CEB.

L'enseignement secondaire octroie également le CEB : en 2005 à 2737 élèves de 1B et 1223 élèves de 2P. En 1B, ces élèves ont un âge moyen de 14 ans et en $2 \mathrm{P}$ de 15 ans.

D ns l'enseignement ordinaire, le Certificat d'Études de Base (CEB) peut être octroyé en fin de $6^{\mathrm{e}}$ primaire mais aussi en $1^{\mathrm{re}}$ et $2^{\mathrm{e}}$ années de l'enseignement secondaire. Dans l'enseignement spécialisé, le CEB est parfois délivré en fin de primaire, parfois au cours du secondaire.

L'obtention du CEB est envisagée ici sous quatre angles. Premièrement, la figure 1 fournit, par sexe, le nombre de CEB délivrés au terme de chaque année d'études et dans chaque type d'enseignement : en 2005, en $6^{\mathrm{e}}$ année primaire de l'enseignement ordinaire, 45512 enfants obtiennent le CEB ; dans le $1^{\text {er }}$ degré du secondaire, c'est le cas pour 2737 élèves de $1 B$ et 1223 de $2 \mathrm{P}$; quant à l'enseignement spécialisé, il délivre très peu de CEB en primaire (183), un peu plus en secondaire (330 pour les garçons et 195 pour les filles).

Deuxièmement, la figure 2 présente l'évolution sur sept ans (19992005) du taux d'obtention du CEB à l'issue de la $6^{e}$ primaire. Sur les 7 années scolaires considérées, le taux moyen d'obtention du CEB des élèves de $6^{\text {e }}$ est de $93,3 \%$, filles et garçons confondus. II varie peu : l'écart le plus grand est de 2,1 points $(94,0 \%$ en $2004,91,9 \%$ en 2000$)$. La différence entre les sexes est à l'avantage des filles : en moyenne, sur les sept ans, $94,0 \%$ des filles obtiennent le CEB en fin de $6^{\mathrm{e}}$ primaire contre $92,6 \%$ des garçons.

Troisièmement, l'obtention du CEB n'étant pas systématique en primaire, une analyse du parcours et de la certification d'élèves inscrits en $5^{\mathrm{e}}$ primaire a été réalisée ${ }^{1}$ (figure 3 ). Sur l'ensemble de la cohorte des 52720 élèves de $5^{e}$ année en 2002-2003, 1406 quittent le primaire en fin d'année scolaire, 1846 redoublent et 49468 passent en $6^{\mathrm{e}}$. Parmi ces derniers, en 2003-2004, 47668 obtiennent le CEB, 731 quittent le primaire et 1069 redoublent la $6^{\mathrm{e}}$. De ce fait en 2004-2005, de la cohorte de départ, 2797 élèves se trouvent encore en $6^{\mathrm{e}}$ primaire, dont 2609 obtiennent le CEB et 188 quittent le primaire sans CEB. On peut ainsi calculer, pour la cohorte de $5^{\mathrm{e}}$ primaire de 2002-2003, un taux de certification. Il est de 93,8 \% en 2 ans et de 95,4 \% en 3 ans. L'approche permet aussi d'évaluer le nombre d'élèves qui, en $5^{\mathrm{e}}$ ou en $6^{\mathrm{e}}$, quittent l'enseignement primaire sans CEB : de la cohorte de départ, 2,9\% quitte à la fin de la $5^{\mathrm{e}}$ et près de $1,7 \%$ à la fin de la $6^{\mathrm{e}}$. On peut émettre l'hypothèse qu'une majorité de ces 2443 élèves sans CEB a gagné l'enseignement secondaire.

Quatrièmement, une étude de l'obtention du CEB est possible par âge pour les deux premières années de l'enseignement secondaire ordinaire. En 2005, 3960 CEB ont en effet été octroyés en 1B et 2P, dont près des trois quarts en $1 \mathrm{~B}$. Les figures 4 et 5 présentent la répartition des âges en fonction de l'année d'études au terme de laquelle les élèves obtiennent ce certificat. En $1^{\text {re }}$ année (figure 4), 53,1 \% des élèves qui reçoivent le CEB ont 14 ans, soit 2 ans de retard sur l'âge normal $\mathrm{d}^{\prime}$ obtention de ce certificat ( 12 ans en fin de $6^{\mathrm{e}}$ primaire). En $2^{\mathrm{e}}$ année (figure 5), 54,0\% des élèves qui obtiennent le CEB ont 15 ans et $17,7 \%$ ont 16 ans. Dans l'enseignement secondaire, le CEB est donc majoritairement délivré en $1 \mathrm{~B}$ et ce, à des élèves ayant 14 ans.

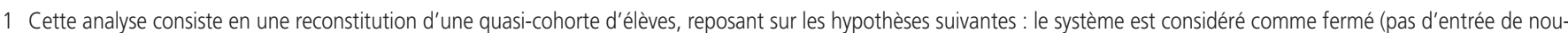
veaux élèves en $5^{\mathrm{e}}$ ou $6^{\mathrm{e}}$ primaire, pas de sortie par émigration ou décès) ; les élèves redoublent au plus une fois en $5^{\mathrm{e}}$ ou en $6^{\mathrm{e}}$ primaire ; les taux de redoublement et de certification sont les mêmes pour tous les élèves d'une année d'études, pour une année scolaire donnée (redoublants ou non).

Pour des informations supplémentaires, voir l'annexe de cet indicateur sur http://www.enseignement.be/prof/dossiers/indicateurs/index.asp.
} 


\subsection{Nombre de CEB délivrés par sexe, selon les types, niveaux d'enseignement et années d'études - Année de certification 2005}

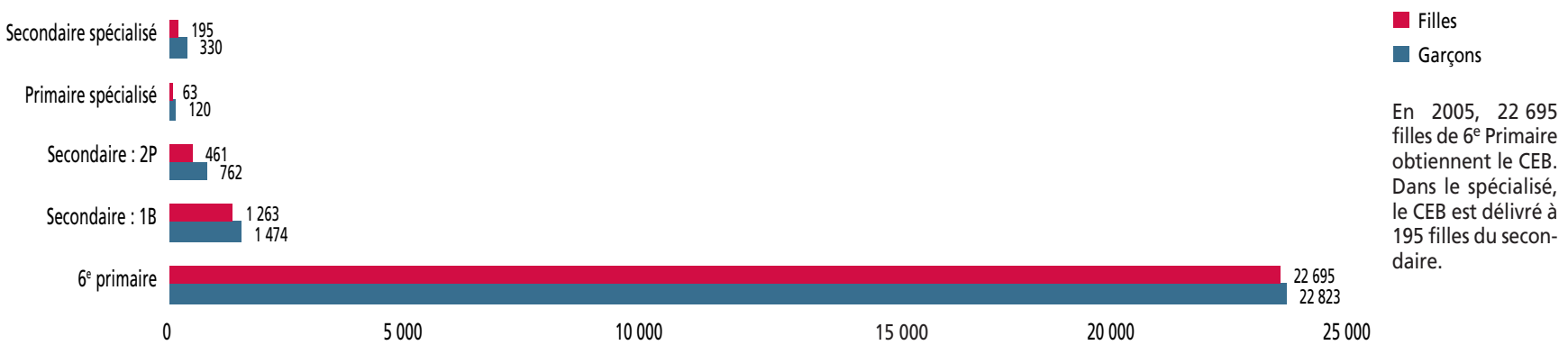

\section{2 Évolution du taux d'obtention du CEB en $6^{e}$ primaire, par sexe, sur la base des effectifs de $6^{e}$ année - Années de certification 1999 à 2005}

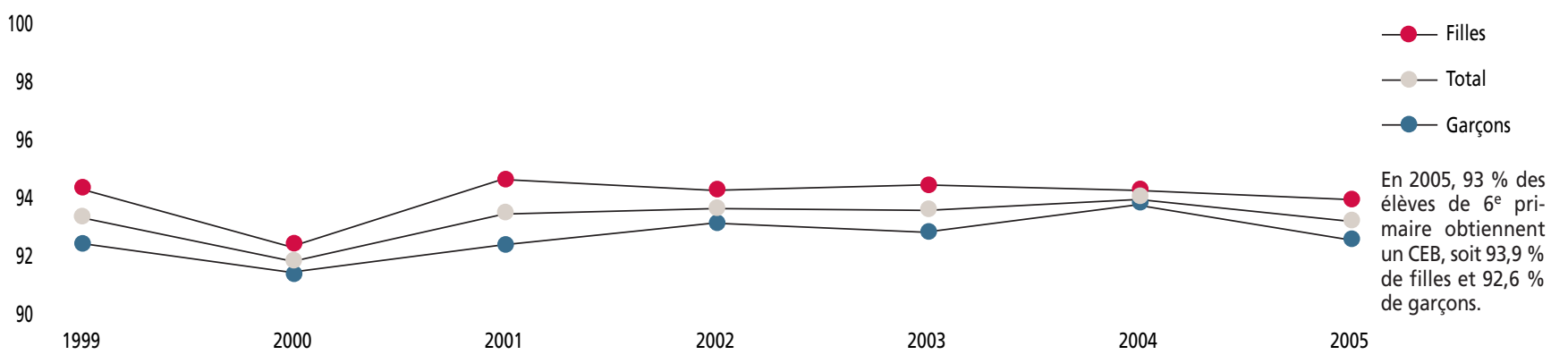

24.3 Parcours des élèves inscrits en $5^{e}$ primaire en 2002-2003 jusqu'à leur sortie du primaire, avec ou sans certificat d'études de base (CEB)

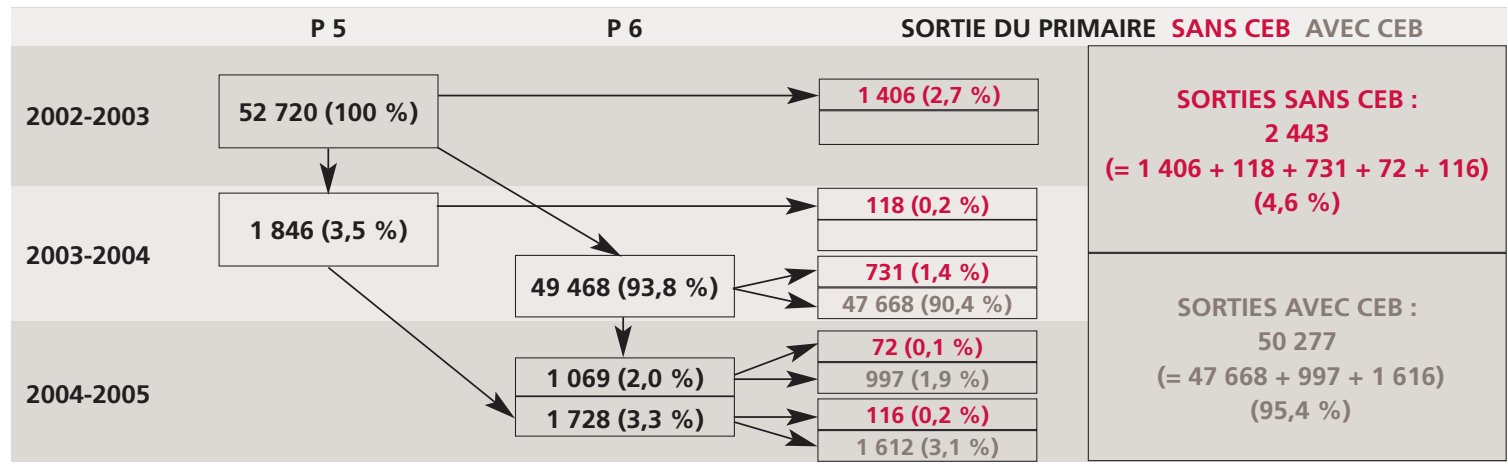

Des 52720 élèves qui se trouvent en $5^{\mathrm{e}}$ primaire en 2002-2003, 50277 obtiennent le CEB et 2443 quittent I'enseignement primaire sans CEB.

\subsection{Obtention du CEB en 1B et 2P - Proportion par âge - Année de certification 2005}
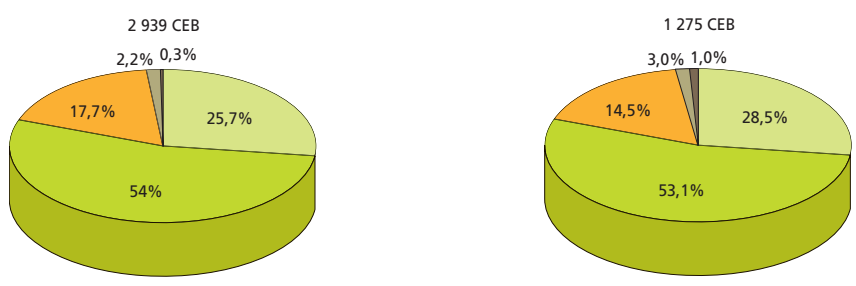

13 ans et moins
14 ans
15 ans
16 ans
17 ans et plus

Parmi les élèves qui obtiennent le CEB en fin de 1B, $53,1 \%$ ont 14 ans. Quand ils obtiennent le CEB en fin de $2 \mathrm{P}$, les élèves ont majoritairement 15 ans (54\%). 


\section{Rythmes scolaires individuels et types de certification en $\sigma^{e}$ année de l'enseignement secondaire ordinaire de plein exercice}

Lorsqu'ils reçoivent un certificat de $6^{\mathrm{e}}$ année du secondaire, nombre d'élèves ont accumulé au moins un an de retard : c'est le cas de plus de la moitié des garçons. Des différences dans ce rythme individuel existent cependant entre les sections d'enseignement : dans la section de transition, une majorité d'élèves sont "à l'heure" alors que l'image est inversée pour la section de qualification.

Par ailleurs, deux traits caractérisent la section de qualification : d'abord, la majorité des élèves de cette section se dote de la double certification (certificat de qualification et certificat d'études ou d'enseignement) ; ensuite, un lien se dessine, tant en technique de qualification qu'en professionnel, entre la double certification et le rythme scolaire individuel, les plus jeunes étant plus enclins à décrocher les deux certificats.

e rythme scolaire individuel est la présence ou l'absence de retard sco-laire. Les élèves qui ont 18 ans ou moins lorsqu'ils reçoivent leur certificat de 6 e sont dits « au moins à l'heure ", ceux qui ont 19 ans sont dits " en retard d'un an » et ceux qui ont plus de 19 ans « en retard de plus d'un an ». Par ailleurs, la finalité de l'enseignement et la certification diffèrent selon la section et la forme d'enseignement. L'enseignement général, artistique de transition et technique de transition délivre le CESS, I'enseignement artistique et technique de qualification délivre le CESS et/ou un CQ6, l'enseignement professionnel permet d'obtenir un CE et/ou un CQ6. Ces certificats n'ont en principe pas les mêmes débouchés: c'est l'éventualité d'une valorisation sur le marché de l'emploi pour les CQ6, I'enseignement supérieur pour le CESS, la $7^{\mathrm{e}}$ professionnelle pour le CE. Les calculs concernant ces certificats ont été effectués pour l'année scolaire 2004-2005.

La figure 1 montre que, toutes sections et formes confondues, sont à l'heure $55,9 \%$ des filles et $43,8 \%$ des garçons qui reçoivent un certificat de fin de $6^{\mathrm{e}}$ année. En distinguant les sections, on voit que les diplômés à l'heure sont majoritaires seulement dans l'enseignement de transition ( $74,6 \%$ des filles et $61,3 \%$ des garçons) et que la différence en faveur des filles à I'heure persiste dans toutes les sections. Le ralentissement du rythme scolaire individuel montre par contre des nuances selon le sexe dans les formes de qualification. Ainsi, au terme du technique de qualification, la majeure partie des filles affiche un retard d' 1 an tandis que la majeure partie des garçons a un retard de 2 ans. En professionnel, les diplômés de $6^{\mathrm{e}}$, filles et garçons, ont le plus souvent un retard de 2 ans.

La figure 2 indique la proportion d'élèves qui, dans les formes qualifiantes, obtiennent les différents types de certificats. On voit que la majorité des élèves a la double possibilité d'accéder à l'emploi (CQ6) et soit à la $7^{\mathrm{e}}$ année professionnelle (CE), soit à l'enseignement supérieur (CESS). Quand un seul certificat est obtenu, il s'agit, en professionnel, du $C E$, en technique de qualification, du CESS. Ceux qui n'obtiennent que le CQ6 sont au plus $4,8 \%$. Ainsi, dans cette section censée préparer à une profession, plus de $95 \%$ des élèves peuvent théoriquement poursuivre des études même si la majorité d'entre eux s'arme aussi de la possibilité d'un accès à l'emploi. Pourtant, une différence apparaît lorsqu'on compare les diplômes obtenus par les filles et par les garçons, surtout dans l'enseignement professionnel : les garçons sont plus nombreux à obtenir la double certification (75,5\% contre $62,2 \%$ ) et les filles plus nombreuses à obtenir le $\mathrm{CE}$ qui peut donner accès à des études supplémentaires. Un profil similaire mais moins marqué apparaît dans l'enseignement technique de qualification.

La possibilité d'accéder au marché de l'emploi offerte par la qualification pourrait, même si cette possibilité n'est pas systématique, lier l'ob- tention d'un CQ6 à l'âge des élèves, les plus âgés étant peut-être davantage pressés de s'engager dans la vie active. La figure 3 détaille donc la figure 2 en fonction du rythme scolaire individuel des élèves. D'abord, quelle que soit la forme et quel que soit le sexe, on voit que même si l'obtention d'un CQ6 seul est rare, c'est pour le groupe des élèves en retard qu'elle est la plus fréquente. Mais le constat est identique pour l'obtention du CE seul ou du CESS seul : comparés aux élèves à l'heure, les élèves en retard sont plus nombreux à recevoir le certificat d'études sans la qualification. Il est donc difficile de lier le retard scolaire à l'obtention préférentielle d'une qualification professionnelle. Tout au plus peut-on dire que les élèves les plus lents ont, plus que les autres, tendance à n'obtenir qu'un seul certificat.

Parallèlement, la figure 3 indique un lien global entre rythme scolaire individuel et double certification. Dans l'enseignement de qualification, la double certification est en effet obtenue par $60,6 \%$ des filles et $73,4 \%$ des garçons à l'heure, par $55,0 \%$ des filles et $64,9 \%$ des garçons en retard d'un an, et par $53,3 \%$ des filles et $54,4 \%$ des garçons en retard de deux ans et plus. Dans l'enseignement professionnel, une même tendance à la double certification des plus jeunes se dessine aussi : reçoivent simultanément le CE et le CQ6 68,2 \% des filles et 83,9\% des garçons à l'heure, $63,6 \%$ des filles et $78,5 \%$ des garçons en retard d'un an, $57,2 \%$ des filles et $68,8 \%$ des garçons en retard d'au moins deux ans.

Glossaire

Sections et formes d'enseignement : dans l'enseignement secondaire ordinaire, deux sections existent. La section de transition regroupe les formes générale, artistique de transition et technique de transition qui préparent à l'enseignement supérieur. La section de qualification regroupe les formes artistique de qualification, technique de qualification et professionnelle, dont la finalité est davantage de préparer à la vie active.

CESS : Certificat d'Enseignement Secondaire Supérieur délivré en 6e année de l'enseignement de transition ou de l'enseignement artistique et technique de qualification. II donne accès à l'enseignement supérieur de type court et de type long.

CE : Certificat d'Études délivré en $6^{e}$ année de l'enseignement professionnel, sanctionnant une année d'études réussie sans obtention du CQ6. Le CE peut donner accès à la $7^{\mathrm{e}}$ année de l'enseignement professionnel.

CQ6 : Certificat de Qualification obtenu au terme d'une $6^{\mathrm{e}}$ année de l'enseignement professionnel ou de l'enseignement artistique et technique de qualification. Le CQ6 est spécifique à l'option choisie et peut être valorisé sur le marché de l'emploi. La présentation de l'examen qui le délivre est optionnelle. 


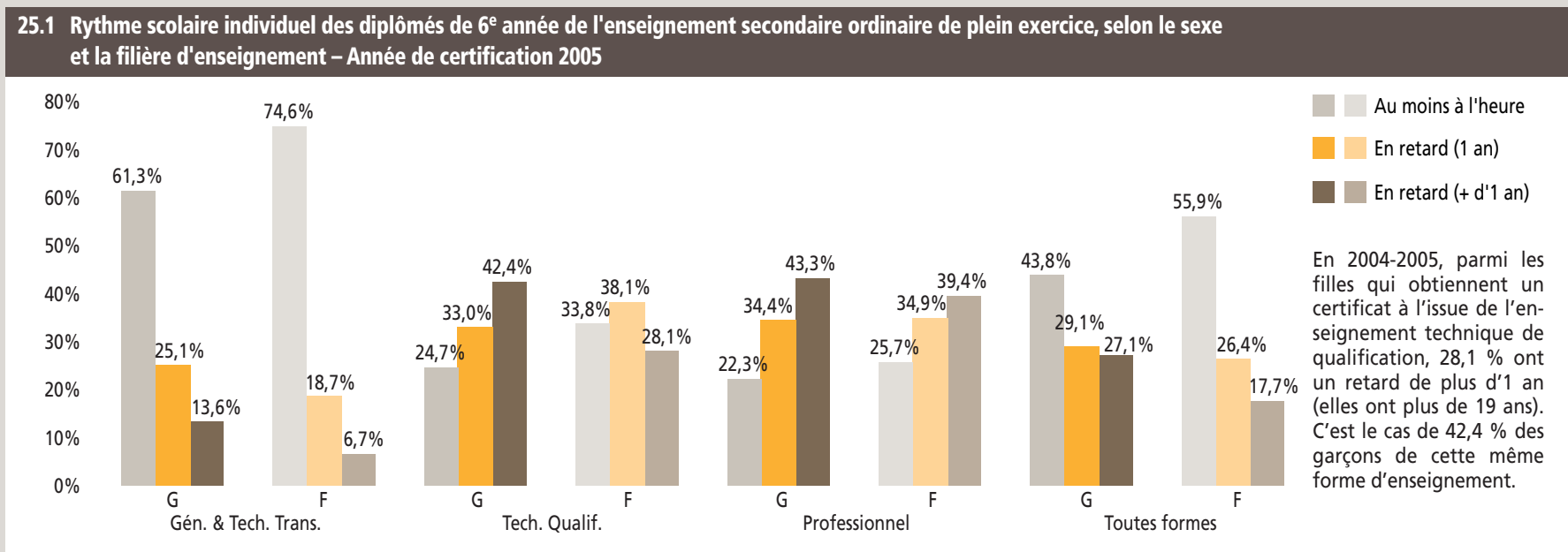

25.2 Types de certificats obtenus en $6^{e}$ année du secondaire, selon le sexe, dans les formes qualifiantes : technique de qualification et professionnel - Année de certification 2005

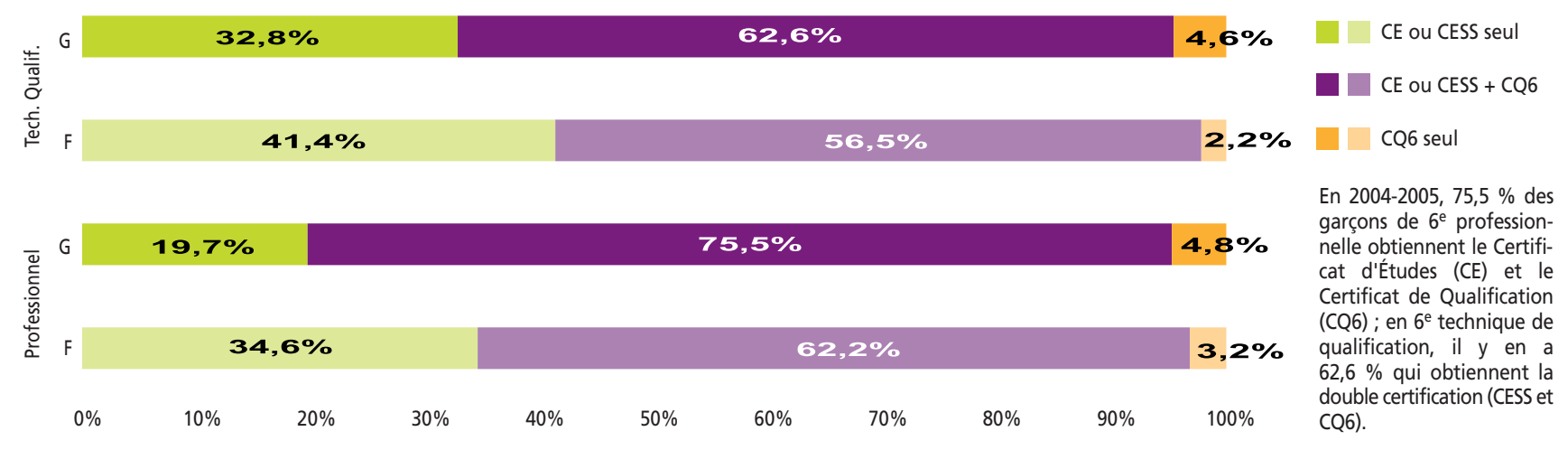

25.3 Rythme scolaire individuel et types de certificats obtenus dans les deux formes qualifiantes, selon le sexe - Année de certification 2005

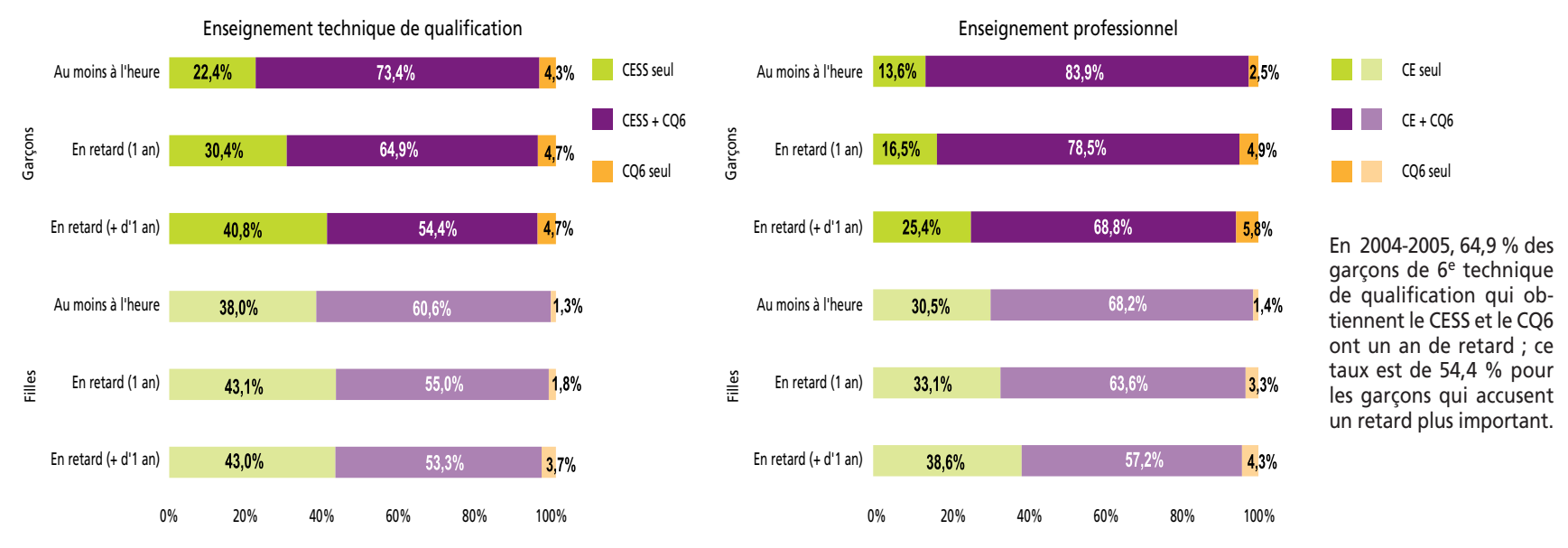




\section{Taux de certification en $6^{e}$ année de l'enseignement secondaire de plein exercice}

En 2005, les taux de certification en $6^{\mathrm{e}}$ année du secondaire de plein exercice, sur base des effectifs de cette année d'études, montrent un ordonnancement des taux de certification entre les formes d'enseignement secondaire, en faveur du général et au détriment du professionnel. Le suivi d'une cohorte d'élèves de $5^{\mathrm{e}}$ secondaire pendant 4 ans révèle des taux de certification de près de $84 \%$ pour toutes les formes d'enseignement confondues, mais un taux de moins de $73 \%$ dans l'enseignement professionnel.

e taux de certification en fin de $6^{\mathrm{e}}$ année de l'enseignement secondaire est ici approché de deux façons : d'abord, par un taux de certification au terme de la $6^{\mathrm{e}}$ sur la base des effectifs y afférents, puis par le suivi d'une cohorte d'élèves durant le $3^{\mathrm{e}}$ degré. Les types de certificats délivrés par les différentes formes d'enseignement de même que l'âge d'obtention de ces certificats font l'objet d'un autre indicateur. Donc, ici, dans les calculs des taux de certification, un élève est comptabilisé comme « certifié » dès lors qu'il obtient au moins un des certificats de $6^{\mathrm{e}}$ existant dans la forme d'enseignement qu'il fréquente. ${ }^{1}$

Sur la base des effectifs de $6^{\mathrm{e}}$ année, la figure 1 fournit, pour 2005, un « instantané » des taux de certification selon les formes d'enseignement. Ces taux s'ordonnent comme suit : I'enseignement général a le plus haut taux de réussite $(93,4 \%$ des élèves) puis viennent le technique de transition $(90,7 \%)$, le technique de qualification $(87,6 \%)$ et enfin le professionnel dont le taux de certification des élèves de $6^{\mathrm{e}}$ est de $81,3 \%$. La différence entre les sexes est à l'avantage des filles dans les quatre formes d'enseignement.

Les figures 2 et 3 présentent les résultats du suivi de la cohorte des élèves présents, en 2001-2002, en $5^{\mathrm{e}}$ année du secondaire de plein exercice, dans son cheminement vers la certification de fin de $6^{\mathrm{e}}$. La figure 2 présente ce parcours pour toutes les sections et formes d'enseignement confondues. En effet, les élèves qui redoublent la $5^{\mathrm{e}}$ changent trop souvent de section et/ou de forme pour qu'une analyse par forme soit possible. En cela, l'enseignement professionnel fait exception puisque, dans le professionnel, la réorientation n'est pratiquement possible qu'à l'intérieur de la forme. La figure 3 lui est donc consacrée. Dans ces figures, les flèches noires désignent les redoublements, les bleues les passages en $6^{e}$, les vertes l'accès aux certificats et les rouges les sorties, sans certificat, de l'enseignement de plein exercice ${ }^{2}$. Entre parenthèses figurent les pourcentages de la cohorte que représentent les nombres bruts.

La figure 2 montre que des 52169 élèves inscrits dans toutes les $5^{\text {es }}$ années du secondaire de plein exercice en 2001-2002, la majorité

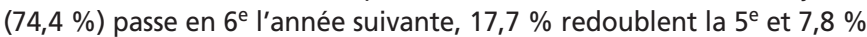
quittent l'enseignement de plein exercice. Les élèves passés en $6^{\mathrm{e}}$ sont 34769 , soit $66,5 \%$ de la cohorte, à décrocher un certificat au terme de l'année scolaire 2002-2003. Durant cette même année scolaire, les sorties du plein exercice sans certificat se dénombrent d'une part en $5^{\mathrm{e}}$ (2 179), d'autre part en $6^{e}(1527)$. Ensuite, de la cohorte cible, l'année scolaire 2003-2004 accueille, en 6e, 2530 redoublants et 7060 élèves de $5^{e}$. Parmi ces 9590 élèves, 8575 décrochent un certificat en fin d'année, alors que 511 élèves quittent le plein exercice sans certificat. Enfin, en 2004-2005, il subsiste de la cohorte 1,0\% d'élèves, c'est-à-dire 504 redoublants de 6 . Parmi eux, 450 obtiennent le certificat cette année-là. Le taux de certification de l'ensemble de la cohorte s'élève donc à $83,7 \%$ pour les 3 années de certification. Quant au taux de sortie sans certificat, il est de $12,3 \%$ en $5^{\mathrm{e}}$ et de $4,0 \%$ en $6^{\mathrm{e}}$. Ainsi, $16,3 \%$ de la cohorte ont quitté, sans certificat, l'enseignement de plein exercice durant le $3^{\mathrm{e}}$ degré du secondaire.

Le suivi des élèves de l'enseignement professionnel de plein exercice fournit les résultats de la figure 3. En 2001-2002, l'effectif est de 10802 élèves en début de $5^{\mathrm{e}}$. L'année suivante, $68 \%$ de ces élèves sont en $6^{\mathrm{e}}$, $20,1 \%$ redoublent la $5^{\mathrm{e}}$ et $11,9 \%$ ont quitté l'enseignement de plein exercice. En 2002-2003, 55,5\% de la cohorte décrochent un certificat alors que 694 élèves de $5^{\mathrm{e}}$ et 688 élèves de $6^{\mathrm{e}}$ arrêtent le cursus sans certificat. En 2003-2004, il reste 2136 élèves de la cohorte en $6^{\mathrm{e}}$ professionnelle : 1738 d'entre eux obtiennent un certificat en fin d'année alors que 246 abandonnent. Enfin, les 152 élèves de la cohorte (1,4\%) qui, en 2004-2005, redoublent la $6^{\mathrm{e}}$ sont 124 à recevoir un certificat en fin d'année et 28 à quitter sans certificat. Le taux de certification pour la cohorte est donc, en professionnel, de $72,8 \%$, le taux d'abandon en $5^{\mathrm{e}}$, de $18,3 \%$ et en $6^{\mathrm{e}}$, de $8,9 \%$. Durant le $3^{\mathrm{e}}$ degré de l'enseignement professionnel, $27,2 \%$ des élèves de la cohorte ont donc quitté l'enseignement de plein exercice sans certificat. ${ }^{3}$

En conclusion, comme l'évaluation des taux de certification en $6^{\mathrm{e}}$ année de l'enseignement secondaire (figure 1), le suivi d'une cohorte $d^{\prime}$ 'élèves du $3^{\mathrm{e}}$ degré (figures 2 et 3 ) révèle un contraste entre l'enseignement professionnel et les autres formes d'enseignement secondaire. Ainsi, toutes formes d'enseignement confondues, près de $84 \%$ des élèves du $3^{\mathrm{e}}$ degré obtiennent un certificat de fin de $6^{\mathrm{e}}$ et, dans l'ensemble, les abandons ne dépassent pas $13 \%$ en $5^{\mathrm{e}}$ et $4 \%$ en $6^{\mathrm{e}}$. Par contre, dans l'enseignement professionnel, moins de $73 \%$ des élèves décrochent un certificat de fin de $6^{\mathrm{e}}$, près $\mathrm{d}^{\prime} 1$ élève sur 5 abandonne en $5^{\mathrm{e}}$ et près $d^{\prime} 1$ élève sur 10 quitte sans certificat la $6^{\mathrm{e}}$. Cette tendance à l'abandon de la forme professionnelle pourrait être liée, d'une part, au fait qu'elle accueille les élèves les plus âgés qui, à partir de 18 ans, ne sont plus soumis à l'obligation scolaire, d'autre part, au fait que ces élèves ont la possibilité de poursuivre leur cursus dans l'enseignement en alternance (CEFA, IFAPME).

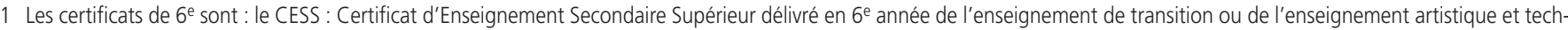
nique de qualification ; le CE : Certificat d'Études délivré en 6e année de l'enseignement professionnel, sanctionnant une année d'études réussie avec ou sans obtention du CQ6 ; le CQ6 : Certificat de Qualification optionnel obtenu au terme d'une 6e année de l'enseignement professionnel ou de l'enseignement artistique et technique de qualification. 2 L'élève abandonne l'enseignement de plein exercice mais peut poursuivre sa scolarité dans l'enseignement en alternance (CEFA, IFAPME).

3 ETNIC - Calculs propres.
} 


\subsection{Certification en $6^{e}$ secondaire, par sexe et par forme d'enseignement, en fonction des effectifs de $6^{e}$ année - Année de certification 2005}

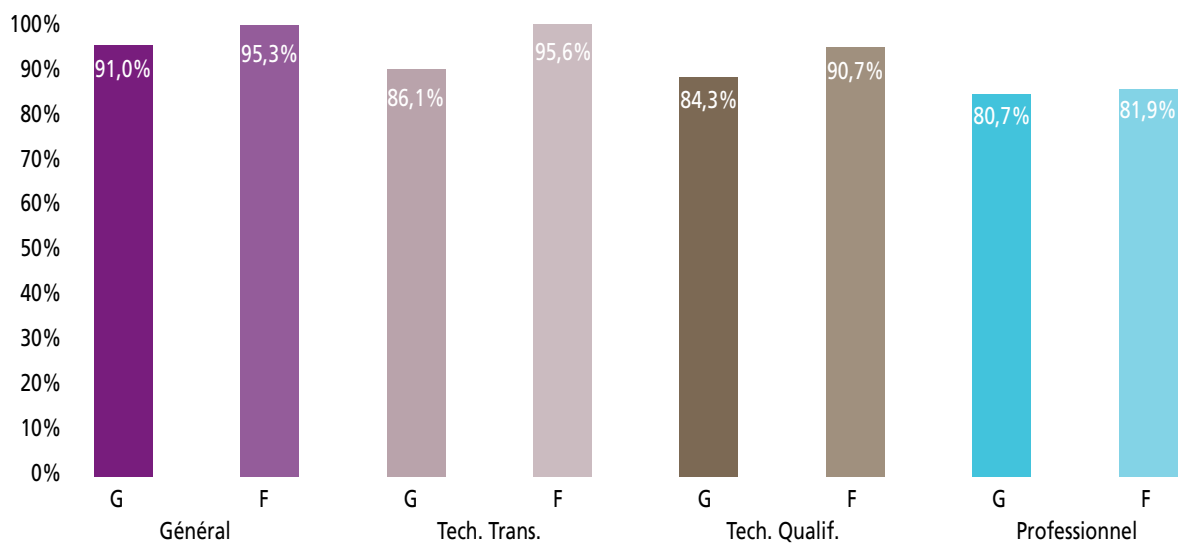

En 2005, 95,3 \% des filles de 6e année de l'enseignement général décrochent un certificat en fin d'année. C'est le cas de 81,9\% des filles de 6 e professionnelle.

26.2 Parcours des élèves présents en $5^{\mathrm{e}}$ secondaire (toutes formes) en 2001-2002 jusqu'à leur sortie du secondaire, avec ou sans certificat de $6^{\mathrm{e}}$

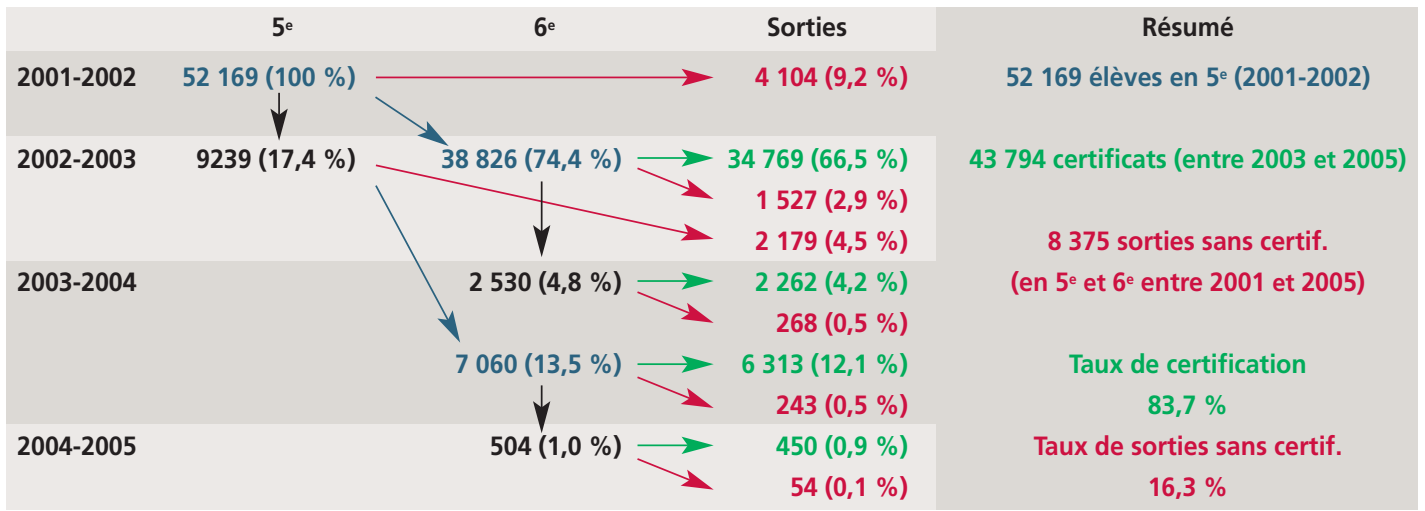

Des 52169 élèves de $5^{e}$ secondaire en 2001 $2002,66,5 \%$ obtiennent un certificat de 6 e en fin d'année 20022003.

Les flèches noires désignent les redoublements, les bleues les passages en $6^{\mathrm{e}}$, les vertes I'accès aux certificats et les rouges les sorties, sans certificat, de l'enseignement de plein exercice.

26.3 Parcours des élèves présents en $5^{e}$ professionnelle en 2001-2002 jusqu'à leur sortie du secondaire, avec ou sans certificat de $6^{e}$

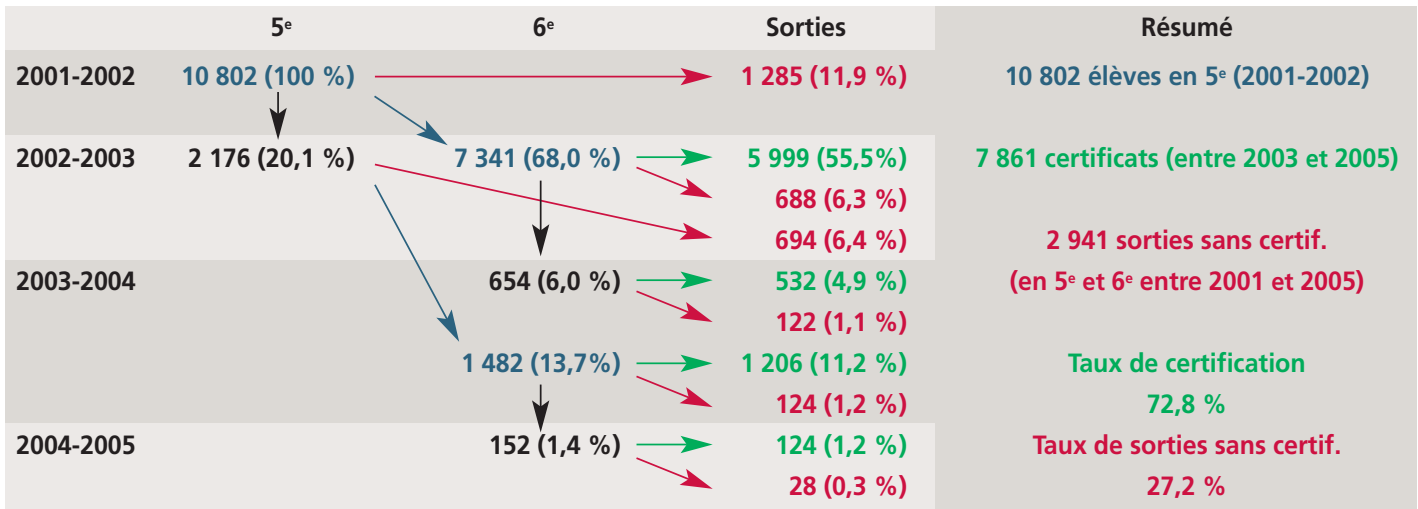

Des 52169 élèves de $5^{\mathrm{e}}$ secondaire en 2001$2002,66,5 \%$ obtiennent un certificat de $6 \mathrm{e}$ en fin d'année 20022003.

Les flèches noires désignent les redoublements, les bleues les passages en $6 \mathrm{e}$, les vertes l'accès aux certificats et les rouges les sorties, sans certificat, de l'enseignement de plein exercice. 
En fin d'année académique 2004-2005, dans l'enseignement supérieur hors universités, le taux de réussite moyen des étudiants de première génération issus du secondaire de la Communauté française ou germanophone et inscrits dans l'enseignement supérieur de type court (Hautes Écoles) est de 41,3\%, contre 40,1\% en fin d'année 2003-2004. Dans l'enseignement supérieur de type long (Hautes Écoles et Instituts Supérieurs d'Architecture), le taux de réussite s'élève à 47,1 \% (40,9\% en fin d'année 2003-2004).

Le taux d'abandon est pour sa part de $21,2 \%$ dans l'enseignement de type court et de $13,2 \%$ dans l'enseignement de type long (respectivement $22,6 \%$ et $12,6 \%$ l'année précédente).

Les variables observées, telles que le sexe, l'âge et la forme d'enseignement secondaire fréquentée influencent fortement ces taux.

\section{Population étudiée et groupes d'étudiants}

L'étude des taux de réussite, de redoublement et d'abandon porte sur les étudiants de première génération dont le titre d'accès à l'enseignement supérieur est le CESS délivré par la Communauté française ou germanophone de Belgique. Cette étude se limite aux Hautes Écoles et aux Instituts Supérieurs d'Architecture et concerne les résultats en fin d'année académique 2004-2005. Les populations étudiantes ont été distinguées en fonction du type d'études supérieures - type court et type long - et réparties en fonction de trois critères: le sexe, l'âge et la forme d'enseignement secondaire suivie.

\section{Enseignement supérieur de type court}

Dans l'enseignement supérieur de type court (figure 1), le taux de réussite des étudiants de première génération est de $41,3 \%$ (contre $40,1 \%$ en fin d'année 2003-2004). À âge et forme d'enseignement secondaire équivalents, les femmes ont un taux de réussite supérieur à celui des hommes. Globalement, le taux de réussite des femmes est de $47,6 \%$ tandis que celui des hommes est de $32 \%$. L'âge - en tant que variable de remplacement du parcours scolaire de l'étudiant - s'inscrit aussi comme un déterminant important de la réussite. À sexe et forme d'enseignement secondaire équivalents, les étudiants "à l'heure " (18 ans et moins) réussissent mieux que les étudiants " en retard " (19 ans et plus). Globalement, le taux de réussite des étudiants " à l'heure " est de 54,2\% tandis que celui des étudiants " en retard " est de $30,9 \%$ (25,3\% chez les étudiants avec au moins deux ans de retard et 35, $1 \%$ chez les étudiants avec seulement un an de retard). Le troisième critère de répartition des étudiants - la forme d'enseignement secondaire suivie - se révèle également discriminant pour le taux de réussite. A sexe et âge équivalent, les étudiants issus du secondaire général (dont le taux global de réussite est de $53,5 \%$ ) réussissent mieux que ceux issus du secondaire technique de transition (39,9\% de réussite), qui, à leur tour, réussissent mieux que ceux issus du secondaire technique de qualification ( $24,5 \%$ de réussite). Ces derniers réussissent mieux que les étudiants issus du secondaire professionnel ( $16,7 \%$ de réussite).

Le taux d'abandon des étudiants de première génération dans l'enseignement supérieur de type court est de 21,2 \%, c'est-à-dire qu'un étudiant sur cinq abandonne l'enseignement supérieur dès la fin de l'année de sa première inscription. Le taux d'abandon est l'image inversée du taux de réussite. Ainsi, à titre d'exemple, les femmes âgées de 18 ans provenant du secondaire général ont un taux d'abandon de 7,9\% alors que les hommes âgés de 20 ans et plus provenant du secondaire technique de qualification ont un taux d'abandon de 40,9\%. Néanmoins, deux différences apparaissent par rapport aux conclusions tirées pour les taux de réussite. La première est qu'à même taux de réussite, les femmes abandonnent plus rapidement que les hommes. La seconde est qu'à même taux de réussite, les étudiants " âgés " (deux ans de retard et plus) abandonnent plus rapidement que les étudiants avec seulement un an de retard.

\section{Enseignement supérieur de type long}

Dans l'enseignement supérieur de type long (figure 2), le taux de réussite des étudiants de première génération est de 47,1 \% (en fin d'année 2003-2004, ce taux n'était que de $40,9 \%$ ), soit un taux supérieur à celui observé dans le type court. Le taux d'abandon dans l'enseignement supérieur de type long n'est, pour sa part, globalement que de $13,2 \%$, soit un taux nettement plus bas que celui du type court (21,2\%). Cette différence est essentiellement due à la présence massive dans l'enseignement supérieur de type long des étudiants " à l'heure » issus du secondaire général. Le taux de redoublement dans le type long ${ }^{1}$ est globalement de 39,7 \% (contre 46,5 \% en fin d'année 2003-2004), soit un taux assez proche de celui observé dans le type court $(37,5 \%)$. Comme dans l'enseignement supérieur de type court, les trois variables - sexe, âge et forme d'enseignement secondaire - sont discriminantes pour les taux de réussite et $d$ 'abandon, $d$ 'une manière très semblable à celle décrite pour l'enseignement supérieur de type court. La principale différence par rapport à l'enseignement supérieur de type court réside dans l'écart entre les hommes et les femmes parmi les étudiants issus de l'enseignement secondaire technique de transition et de qualification où il apparaît que les hommes réussissent sensiblement mieux que les femmes (il convient cependant de souligner que les effectifs d'étudiants issus de ces deux formes d'enseignement secondaire sont très réduits dans l'enseignement supérieur de type long).

\section{Glossaire}

Étudiant de première génération : étudiant qui s'inscrit pour la première fois dans un enseignement supérieur.

Taux de réussite : pourcentage d'étudiants passant, l'année académique suivante, dans une année d'études supérieure (dans ce cas, en $2^{\mathrm{e}}$ année).

Taux de redoublement : pourcentage d'étudiants demeurant, l'année académique suivante, dans la même année d'études (dans ce cas, en 1 ère année).

Taux d'abandon : pourcentage d'étudiants non présents l'année académique suivante en Hautes écoles ou Instituts Supérieurs d'Architecture.

1 II est à noter qu'une partie des ces redoublants se réorientent vers l'enseignement supérieur de type court. 


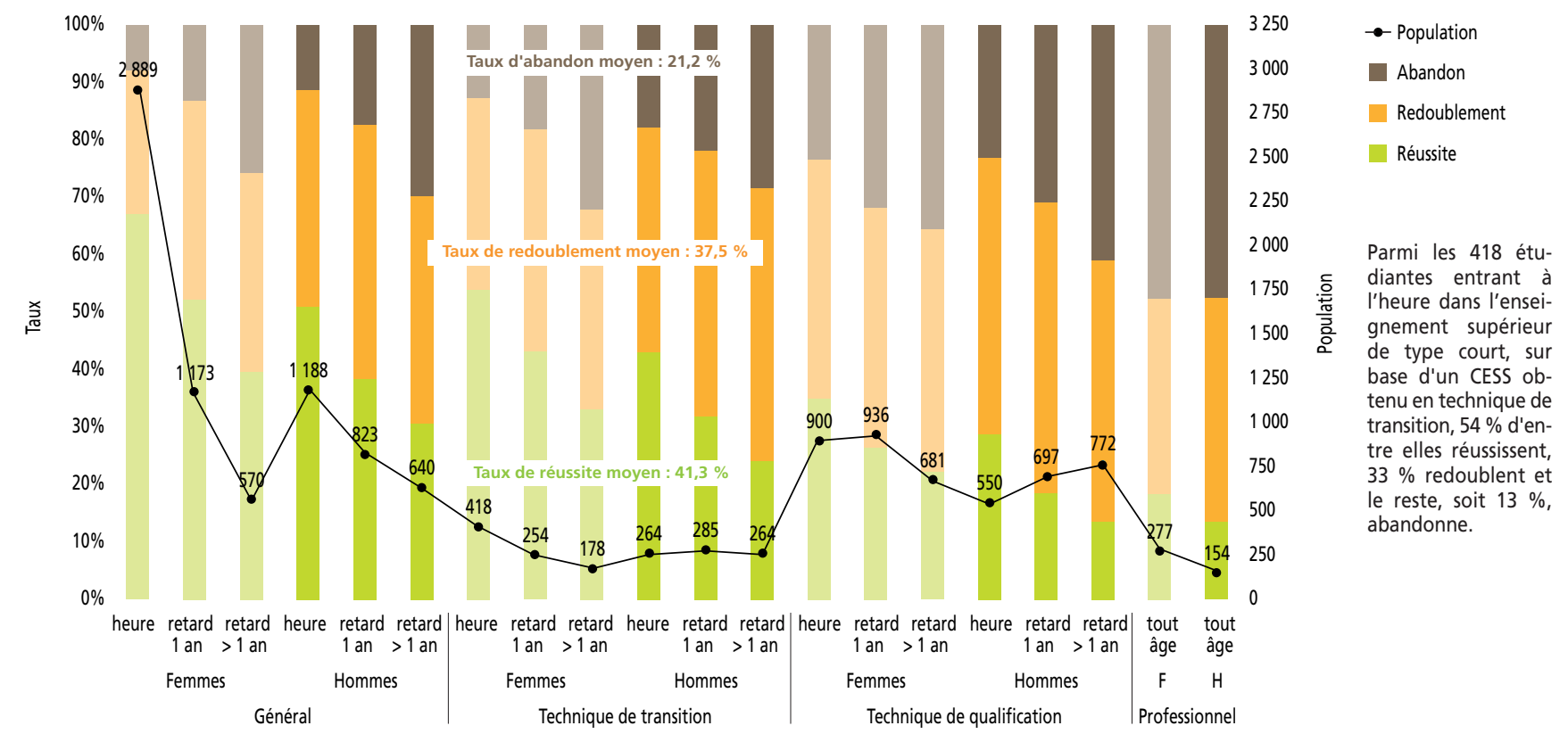

27.2 Résultats en fin d'année académique 2004-2005 des étudiants de $1^{\text {re }}$ génération dont le titre d'accès est le CESS de la Communauté française ou germanophone - Enseignement de type long en Hautes écoles et Instituts Supérieurs d'Architecture

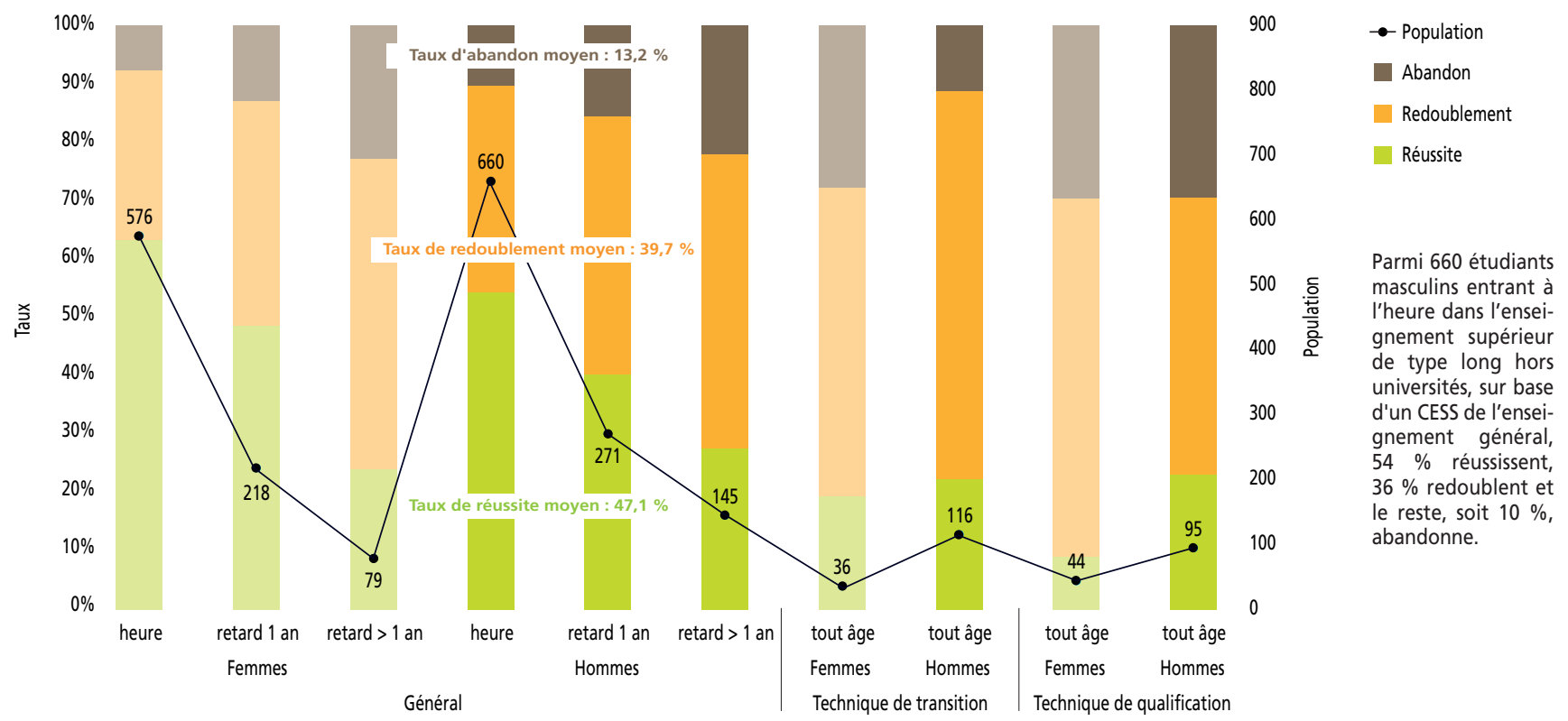


En fin d'année académique 2004-2005, dans l'enseignement universitaire, le taux de réussite moyen des étudiants de première génération issus du secondaire de la Communauté française ou germanophone est de $44,6 \%$ tandis que le taux d'abandon (sortie de l'enseignement universitaire) est de $21,4 \%$ (contre respectivement $42,8 \%$ et $24,4 \%$ en fin 2003-2004). Un tiers des étudiants de première génération de l'enseignement universitaire redoublent donc au sein du système universitaire leur première année d'études supérieures.

Parmi les variables qui peuvent être observées, l'âge ainsi que la forme d'enseignement secondaire fréquentée s'inscrivent comme les principaux facteurs de la réussite bien que le sexe apparaisse aussi comme un élément déterminant.

e présent indicateur complète l'étude des taux de réussite, de redoublement et d'abandon dans l'enseignement supérieur. II porte sur les étudiants de première génération entrant dans l'enseignement universitaire en Communauté française. Le titre d'accès à l'enseignement supérieur des étudiants observés est le CESS délivré par la Communauté française ou germanophone de Belgique. L'indicateur s'appuie sur les résultats de fin d'année académique 2004-2005. Les populations étudiantes ont été réparties selon trois critères : le sexe, l'âge et la forme d'enseignement secondaire suivie.

Dans l'enseignement universitaire, le taux de réussite global des étudiants de première génération (détenteurs d'un CESS délivré par la Communauté française ou germanophone) est de 44,6\% (en fin d'année 2003-2004, ce taux était de $42,8 \%$ ), soit un taux de réussite situé, cette année, entre le taux de réussite de l'enseignement supérieur hors universités (SHU) de type court, de $41,3 \%$, et celui du SHU de type long, de $47,1 \%$.

Comme dans le SHU, les femmes ont un taux de réussite supérieur à celui des hommes, à âge et forme d'enseignement secondaire équivalents. Globalement, le taux de réussite des femmes est de $49,1 \%(52,5 \%$ dans le SHU de type long et $47,6 \%$ dans le SHU de type court) tandis que celui des hommes est de 39,4\% (43,2 \% dans le SHU de type long et $32 \%$ dans le SHU de type court). Dans l'ensemble, l'écart entre les taux de réussite des hommes et des femmes est donc moindre dans l'enseignement supérieur de niveau universitaire que dans le SHU de type court, de l'ordre de $9 \%$ dans le premier cas, de l'ordre de $15 \%$ dans le second. Cet écart moindre se confirme si on compare les groupes de populations masculines et féminines à âge et forme d'enseignement équivalents.

L'âge - en tant que variable de remplacement du parcours scolaire de l'étudiant - s'inscrit comme le principal déterminant de la réussite dans l'enseignement universitaire. Ainsi, les étudiant(e)s de 18 ans et moins (" à l'heure ") issus de l'enseignement secondaire général ont un taux de réussite de $54,1 \%$ tandis que les étudiant(e)s de 19 ans (" en retard d'un an») ont un taux de réussite de 34,3\%. Chez les étudiant(e)s de 20 ans et plus (" en retard de plus d'un an »), le taux de réussite n'est plus que de $15,9 \%$. Il est à noter que des écarts quasi similaires s'observent dans le SHU de type long et de type court.

Le troisième critère de répartition des étudiants - la forme d'enseignement secondaire suivie - se révèle également très discriminant pour le taux de réussite quoiqu'il ne concerne qu'un nombre réduit d'étudiants dans l'enseignement universitaire. Les étudiants issus du secondaire général représentent $92,6 \%$ de l'effectif des étudiants de première génération dans l'enseignement universitaire, tandis que les étudiants issus du technique de transition ne représentent que $4,3 \%$ de l'effectif, et les étudiants issus du technique de qualification 3,1\%. Les étudiants issus du secondaire général présentent un taux de réussite de $47,1 \%$, tandis que ceux issus du secondaire technique de transition ont un taux de réussite de $16 \%$, et ceux issus du secondaire technique de qualification de $8,5 \%$.

Le taux d'abandon des étudiants de première génération dans l'enseignement universitaire s'élève à $21,4 \%$, soit un taux largement supérieur à celui observé dans l'enseignement supérieur hors universités de type long $(13,2 \%)$ alors que la composition de la population des deux types d'enseignement - en fonction du sexe, de l'âge et de la forme d'enseignement secondaire - est sensiblement la même. L'explication de cette différence entre les deux taux d'abandon réside en réalité essentiellement dans la réorientation des étudiants. Dans le SHU de type long, une partie des étudiants échouant en première année se réorientent vers le SHU de type court et ont donc été considérés comme des redoublants du SHU. Ils viennent " renforcer » le taux de redoublement dans le SHU de type long. Dans l'enseignement universitaire, une partie des étudiants échouant en première année se réorientent vers le SHU et, dès lors, sont considérés comme des " abandons " (l'indicateur étant centré sur l'enseignement universitaire). Ils « renforcent » donc le taux d'abandon dans l'enseignement universitaire mais ne sont pas de véritables sorties de l'enseignement supérieur considéré dans son intégralité.

\section{Glossaire}

Étudiant de première génération : étudiant qui s'inscrit pour la première fois dans un enseignement supérieur.

Taux de réussite : pourcentage d'étudiants passant, l'année académique suivante, dans une année d'études supérieure (dans ce cas, en $2^{\mathrm{e}}$ année).

Taux de redoublement : pourcentage d'étudiants demeurant, l'année académique suivante, dans la même année d'études (dans ce cas, en $1^{\text {re }}$ année).

Taux d'abandon : pourcentage d'étudiants non présents l'année académique suivante dans l'enseignement universitaire. 


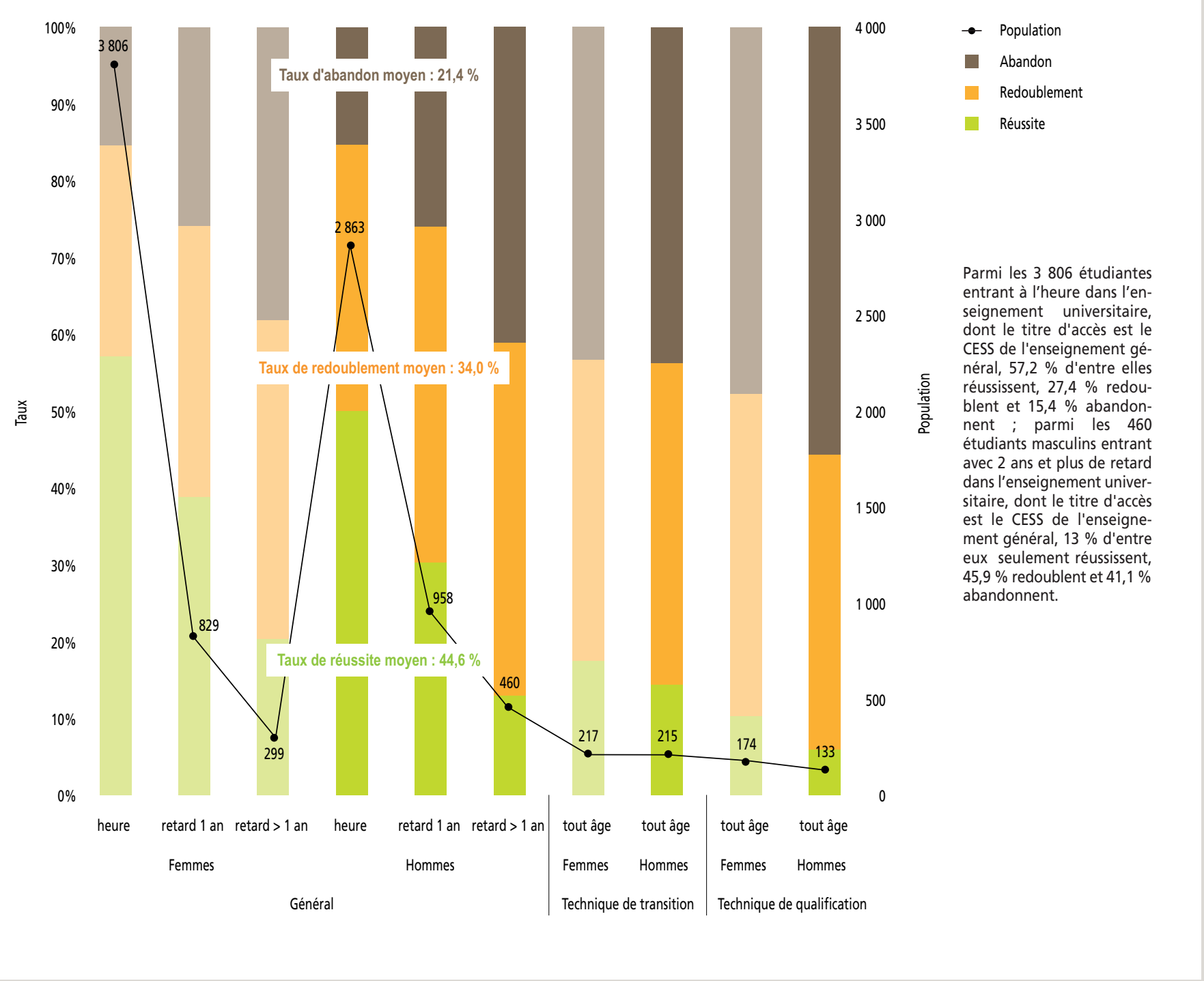




\section{Formation initiale des enseignants de l'enseignement obligatoire}

Après une chute de fréquentation des filières de formation initiale des enseignants dans la deuxième moitié des années 1990 , passant de 17312 inscrits en 1994-1995 à 12312 inscrits en 1998-1999, on observe actuellement une augmentation des inscriptions dans ces matières (16 777 inscrits en 2004-2005 et 16953 inscrits en 2005-2006).

Cette tendance à la réorientation du choix des étudiants vers les filières de formation initiale des enseignants s'observe depuis 2002-2003 dans toutes les sections (à l'exception de la normale technique moyenne), et plus particulièrement dans les sections normales primaire et secondaire dont le nombre d'inscriptions, tant en 2004-2005 qu'en 2005-2006, dépasse les pics de 1994-1995 et 1995-1996. On constate néanmoins en 2005-2006 un repli ou une stagnation des effectifs en normale primaire et en AESS.

Sur la période observée, le nombre de diplômés dans les filières de formation initiale des enseignants est passé de 3395 diplômés, en 1993 , à 4 239 diplômés, en 1997, pour se réduire à 3042 diplômés en 2002 et remonter ensuite à 3526 diplômés en 2004 et 3967 diplômés en 2005 . La croissance des inscriptions permet en outre de prévoir encore une croissance du nombre des diplômés entre 2006 et 2008.

a formation initiale des enseignants en Communauté française relève Lde l'ensemble des institutions organisant l'enseignement supérieur :

- I'enseignement de niveau universitaire (les universités ainsi que les Hautes Écoles et les Écoles Supérieures des Arts organisant l'enseignement de type long) organise et délivre l'agrégation de l'enseignement secondaire supérieur (AESS) formant les enseignants du secondaire supérieur ;

- I'enseignement de type court de la catégorie pédagogique des Hautes Écoles forme les instituteurs préscolaires et primaires ainsi que les agrégés de l'enseignement secondaire inférieur; dans le type court du domaine de la musique, les Écoles Supérieures des Arts forment également des agrégés de l'enseignement secondaire inférieur (AESI);

- I'enseignement de promotion sociale du niveau supérieur de type court organise et délivre le certificat d'aptitude pédagogique (CAP) destiné à la formation des enseignants des cours techniques et de pratique professionnelle ${ }^{1}$

Dans la première moitié des années 1990, on observe une croissance des inscriptions (figure 1) dans les différentes filières d'enseignement de la formation initiale des enseignants, à l'exception de la section normale technique moyenne, d'emblée extrêmement peu fréquentée et en baisse continue sur toute la période observée. Ainsi, les étudiants en section normale préscolaire passent de 2978 inscrits en 1992-1993 à 4136 inscrits en 1995-1996, les étudiants en section normale primaire passent de 4090 à 5447 inscrits sur la même période ; de même, les étudiants en section normale secondaire passent de 3867 inscrits en 1992-1993 à 4578 inscrits en 1994-1995 et les étudiants inscrits en AESS voient leur nombre passer de 2643 à 3099 unités sur la même période. La période 1992-1993 à 1995-1996 correspond aussi à une période de croissance globale de la population dans l'enseignement supérieur, principalement dans les Hautes Écoles qui voient leur population passer de 57731 étudiants à 64893 étudiants.

Dans la seconde moitié des années 1990, alors que la population étudiante continue d'augmenter au sein des Hautes Écoles (67 904 étudiants en 1999-2000) et se met à décroître dans les universités (60 300 étudiants en 1999-2000), on assiste à une chute générale de la fréquentation des domaines de la formation initiale des enseignants, dont le total des ins- criptions, tous domaines confondus, passe de 17308 étudiants en 19951996 à 12404 étudiants en 1999-2000, soit une diminution de $28 \%$ en quatre années. La détérioration du climat social, stigmatisée par plusieurs grèves, ainsi que la polémique sur l'excès d'étudiants dans les métiers de l'enseignement $n$ 'y sont certainement pas étrangères.

Au début des années 2000, différentes mesures et plans d'action parmi lesquels les augmentations salariales, l'alignement du salaire des instituteurs de l'enseignement fondamental et des régents de l'enseignement secondaire inférieur, les décrets en faveur de la revalorisation du métier d'enseignant et les campagnes médiatiques axées sur l'existence d'une pénurie d'enseignants dans les années à venir - ont contribué au redressement du nombre d'inscriptions. En 2003-2004, dans la section normale primaire, le nombre d'inscrits est passé à 5857 étudiants, soit au-dessus du pic de 1995-1996, et cette croissance s'est poursuivie en 2004-2005 avec 6022 inscriptions, pour quelque peu se réduire en 2005-2006 à 5809 inscriptions; dans la section normale secondaire, le nombre d'inscrits en 2004-2005 (5 086 étudiants) a dépassé le pic de 1994-1995 (4 578 inscriptions), cette hausse des inscriptions s'est poursuivie en 2005-2006 avec 5305 étudiants ; dans la section normale préscolaire et au niveau des AESS, une reprise de la croissance des effectifs est apparue depuis 2002-2003 mais le nombre d'inscriptions (3 429 étudiants en normale préscolaire et 2246 étudiants en AESS, en 2005-2006) demeure encore largement sous les maxima atteints au milieu des années 1990 (4 136 étudiants en normale préscolaire en 1995-1996 et 3099 étudiants en AESS en 1994-1995).

L'observation des diplômes délivrés (figure 2) indique qu'en 1993 (fin d'année académique 1992-1993), 1019 AESS et 2376 graduats avaient été délivrés. Sur la période étudiée, le maximum de délivrance d'AESS a été atteint en 1995 (1 309 AESS) et le maximum de délivrance de graduats a été atteint en 1997 (3 308 graduats). En 2005, dernière année observée, 1088 AESS et 2879 graduats ont été délivrés. L'évolution des inscriptions décrite précédemment permet de prévoir ${ }^{2}$ (en pointillés dans la figure 2) une croissance du nombre des gradués ${ }^{3}$ à I'horizon 2008 (estimation de 3282 graduats délivrés).

\footnotetext{
1 Données non disponibles.

2 ETNIC - Estimations propres.

3 Bachelier, selon la nouvelle terminologie.
} 


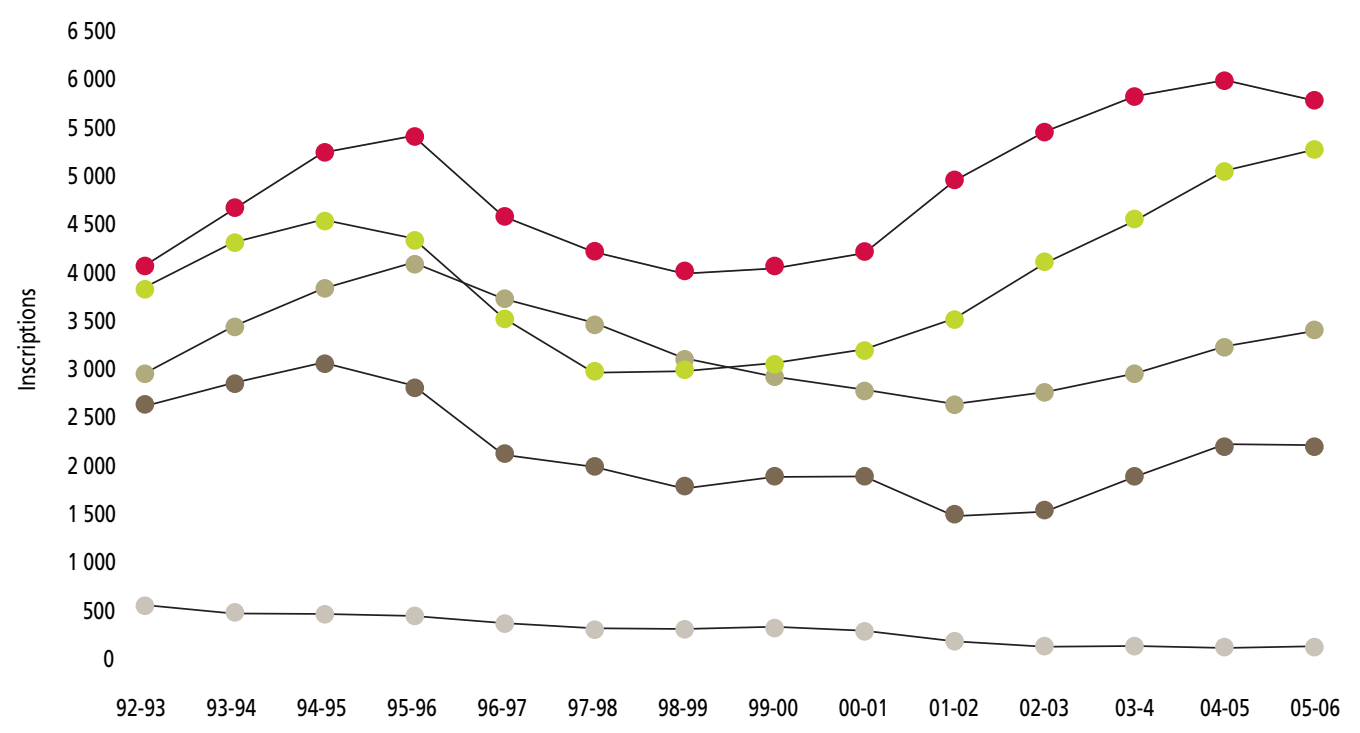

\subsection{Nombre de diplômes délivrés en AESS et dans les sections normales (en fin d'année académique)}

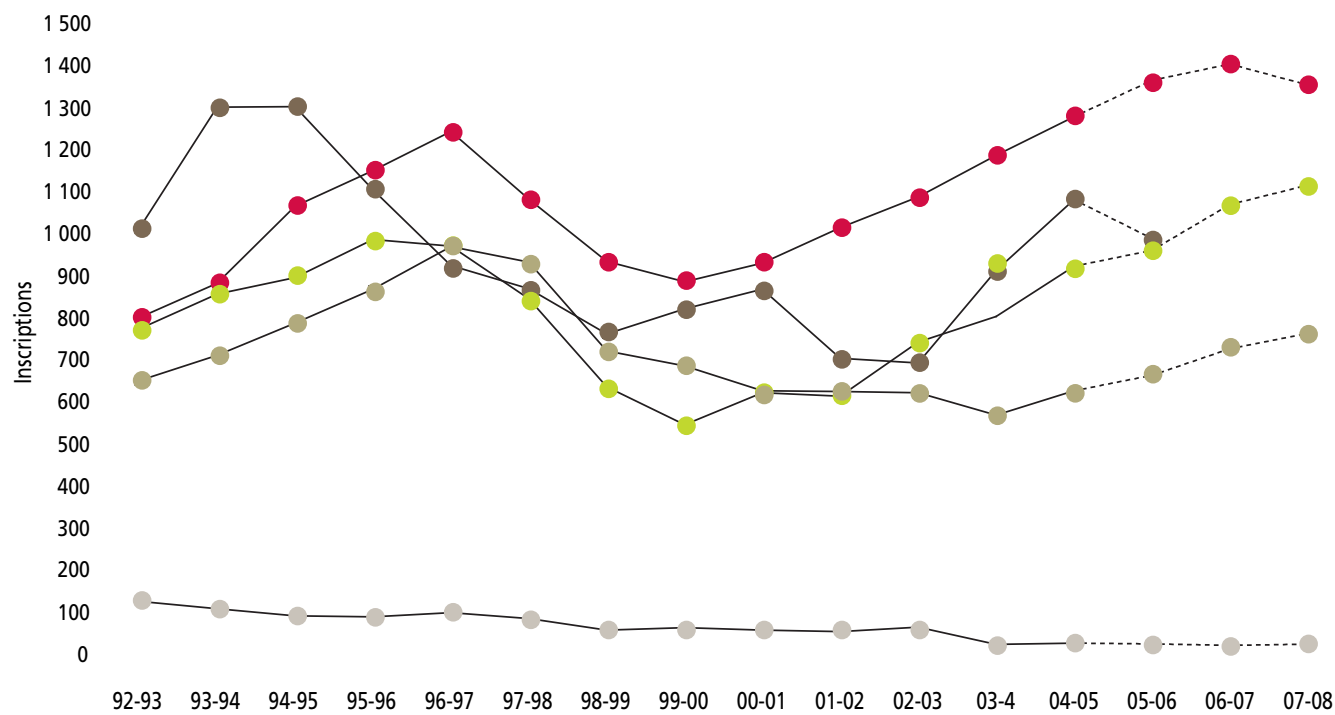

- AESS
- Normale préscolaire
- Normale primaire
- Normale secondaire
- Normale technique moyenne

En 2005-2006, le nombre d'inscriptions dans la section normale primaire est de 5809 étudiants alors que ce nombre était de 4090 étudiants en 1992-1993 et 5447 étudiants en 1995-1996.

- Normale préscolaire

- Normale primaire

- - Normale secondaire

- - Normale technique moyenne

En fin d'année 2004-2005, le nombre de diplômes d'instituteurs primaires délivrés est de 1285 , contre 807 en 1992-1993; les perspectives pour l'année académique 2007-2008 prévoient 1360 diplômes d'instituteurs primaires délivrés. 


\section{A Nombre de charges enseignantes de l'enseignement ordinaire fondamental et secondaire}

De 1994-1995 à 2005-2006, la population enseignante, exprimée en équivalents temps-plein, est restée stable dans l'enseignement fondamental ordinaire et a chuté de $8 \%$ dans l'enseignement secondaire ordinaire

a population étudiée concerne les enseignants calculés en " équivaLlents temps-plein " ${ }^{1}(\mathrm{ETP})$. Les " équivalents temps-plein " pris en considération sont ceux rémunérés par la Communauté française ${ }^{2}$, que la charge d'enseignement soit prestée ou non. Toutefois, les personnes ayant opté pour un départ ${ }^{3}$ anticipé à la retraite ont été exclues des statistiques, leur choix étant irréversible.

Les figures 1 et 3 présentent l'évolution des effectifs enseignants (ETP) par niveau d'enseignement. A titre indicatif, cette évolution est mise en parallèle avec celle des élèves (figures 2 et 4). Eu égard à la population étudiée, il est primordial de garder à l'esprit que le rapport " élèves / enseignant (ETP) " proposé ci-dessous ne représente pas un taux d'encadrement de terrain, vu la notion « d'équivalents tempsplein " pris en compte.

En ce qui concerne l'enseignement fondamental ordinaire, la figure 1 montre l'évolution du nombre d'enseignants (ETP) recensés par réseau, de 1994-1995 à 2005-2006. Tous réseaux confondus, ce nombre est resté stable, il est passé de 31040 à 31298 ETP sur les années étudiées. En revanche, sur cette période, le réseau de la Communauté a connu une décroissance importante (-23 \%) de son personnel enseignant. Ces mêmes effectifs ont très légèrement diminué dans le libre $(-2 \%)$ et ont augmenté dans l'officiel communal et provincial (+ $9 \%)$. Pour ce niveau d'enseignement, la figure 2 met en regard l'évolution des enseignants (ETP) et celle des élèves, de 1994-1995 à 2005-2006. Elle fait logiquement apparaître un rapport constant entre les deux populations, l'encadrement étant réglementé sur la base du nombre d'élèves présents dans le système. Ce rapport s'élève à environ 16 élèves par enseignant (ETP) dans le réseau libre et à environ 15 élèves dans les autres réseaux. Cette différence provient du fait que les charges comprennent celles que gé- nèrent les professeurs de cours philosophiques. En effet, le poids des cours philosophiques ne pèse pas de la même façon dans l'enseignement libre confessionnel et dans l'enseignement officiel. Dans le libre confessionnel, les deux périodes hebdomadaires de religion sont assurées par un enseignant, dans l'officiel, elles sont assurées par autant d'enseignants qu'il y a de cours philosophiques demandés par les parents. Par conséquent, six cours philosophiques potentiels peuvent engendrer la présence de six enseignants en parallèle, ce qui a un impact sur le ratio élèves/enseignants.

En ce qui concerne l'enseignement secondaire ordinaire, la figure 3 représente l'évolution du nombre d'enseignants (ETP) par réseau, de 1994-1995 à 2005-2006. Tous réseaux confondus, le nombre d'enseignants (ETP) a diminué de $8 \%$ au cours de cette période, chutant de 40229 à 36939 ETP. C'est au sein du réseau de la Communauté et du réseau officiel communal et provincial que sont enregistrées les plus fortes diminutions de personnel enseignant (ETP), respectivement -19\% et $-12 \%$. En revanche, si les effectifs enseignants (ETP) du libre ont également diminué de $1 \%$ en moyenne sur les années étudiées, ils augmentent depuis 2000-2001. La figure 4 compare l'évolution du nombre d'enseignants (ETP) à celle du nombre d'élèves pour ce niveau d'enseignement par réseau, depuis 1997-19984. Ce ratio varie de 9 élèves par enseignant (ETP) dans l'officiel à 10 élèves dans le libre. La comparaison de ces ratios doit également être nuancée en tenant compte du poids relatif des charges des professeurs des cours philosophiques par réseau, tel qu'expliqué précédemment. Une autre hypothèse, en ce qui concerne l'enseignement subventionné, est la part d'élèves plus importante dans les sections qualifiantes (cf indicateur 6) qui bénéficient d'un taux d'encadrement plus favorable.

1 Un « équivalent temps-plein » représente l'unité de mesure d'une charge budgétaire.

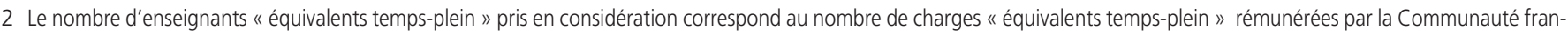
çaise au 15 janvier de l'année scolaire. II prend en compte non seulement les enseignants en poste mais également ceux qui sont détachés, en mission, en congé de maternité, etc.

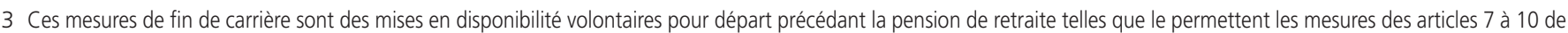
l'Arrêté Royal du 31 mars 1984.

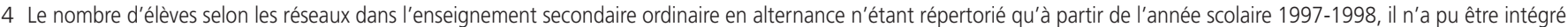
à l'évolution du total des élèves de l'enseignement secondaire ordinaire qu'à partir de l'année scolaire 1997-1998. 
30.1 Évolution du personnel enseignant (ETP) de l'enseignement fondamental ordinaire par réseau - Années 1994-1995 à 2005-2006

- Communauté française Libre Communal + Provincial

18000

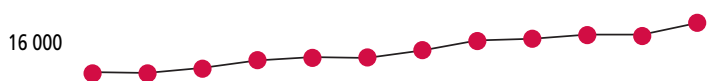

14000

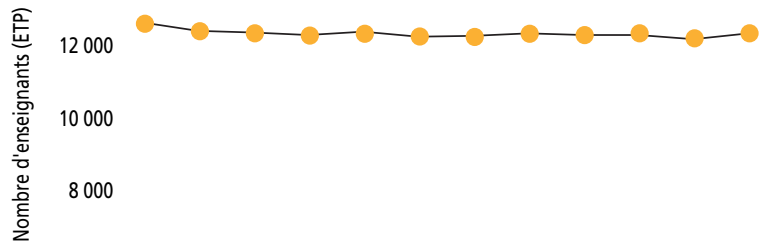

6000

4000

2000

94-95 95-96 96-97 97-98 98-99 99-00 00-01 01-02 02-03 03-04 04-05 05-06

En 2005-2006, le nombre d'équivalents temps-plein du personnel enseignant s'élève à 12213 au niveau fondamental du réseau libre.

\section{3 Évolution du personnel enseignant (ETP) de l'enseignement} secondaire ordinaire par réseau - Années 1994-1995 à 2005-2006

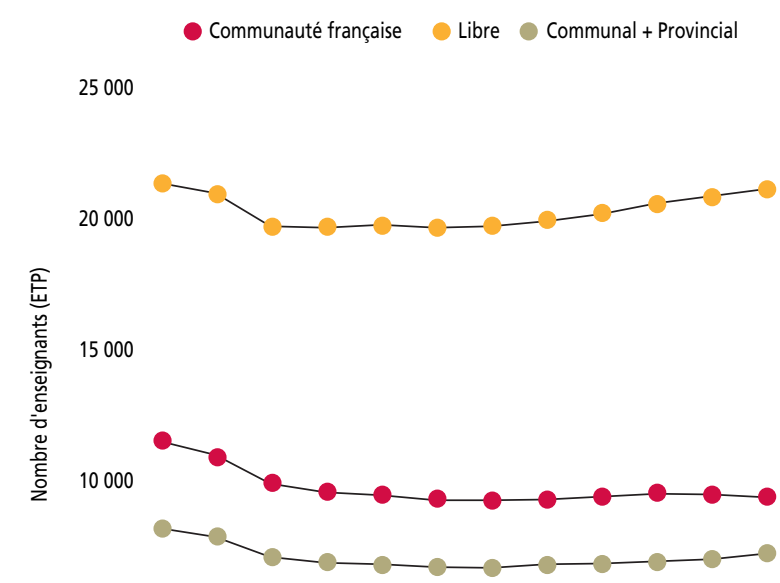

5000

$\begin{array}{lllllllllll}94-95 & 95-96 & 96-97 & 97-98 & 98-99 & 99-00 & 00-01 & 01-02 & 02-03 & 03-04 & 04-05\end{array}$ En 2005-2006, le nombre d'équivalents temps-plein du personnel enseignant s'élève à 9106 au niveau secondaire du réseau de la Communauté française.
30.2 Évolution du ratio « élèves / enseignants (ETP) ॥ de l'enseignement fondamental ordinaire par réseau - Années 1994-1995 à 2005-2006

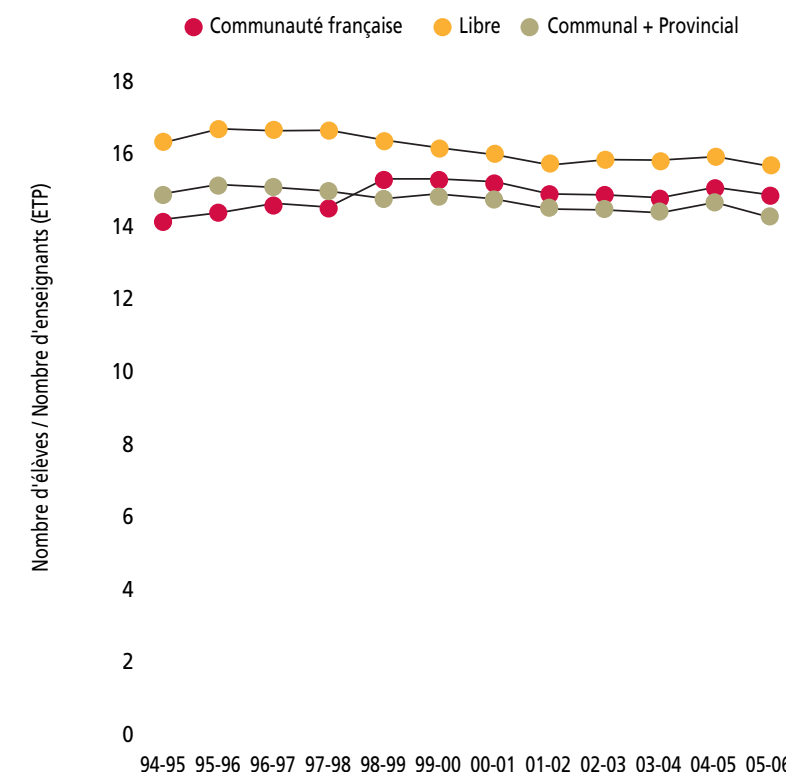

En 2003-2004, le ratio " élèves / enseignants (ETP) » est de 14,5 pour le réseau officiel subventionné « communal + provincial ».

\section{4 Évolution du ratio « élèves / enseignants (ETP) ॥ de l'enseignement} secondaire ordinaire par réseau - Années 1997-1998 à 2005-2006

Communauté française Libre Communal + Provincial

18

16

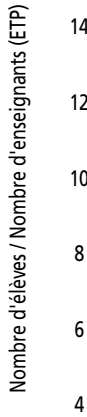

$\begin{array}{lllllllllllllll}94-95 & 95-96 & 96-97 & 97-98 & 98-99 & 99-00 & 00-01 & 01-02 & 02-03 & 03-04 & 04-05 & 05-06\end{array}$ En 2003-2004, le ratio " élèves / enseignants (ETP) » est de 8,5 pour le réseau officiel subventionné " communal + provincial ». 
En 2006, en termes d'âge moyen, le personnel enseignant du niveau fondamental ordinaire est 4 ans plus jeune que celui du niveau secondaire ordinaire.

Plus de la moitié des enseignants ont plus de $\mathbf{4 1}$ ans. Le vieillissement des enseignants est plus marqué au niveau secondaire ordinaire.

La plupart des enseignants profitent des possibilités de retraite anticipée proposées à partir de 55 ans, quittant ainsi l'enseignement avant l'âge minimal de départ à la retraite et ce, d'autant plus tôt au niveau fondamental.

e personnel de l'enseignement pris en considération correspond à Ll'ensemble des enseignants rémunérés par la Communauté française, que la charge d'enseignement soit prestée ou non ${ }^{1}$, à l'exception des personnes ayant opté pour un départ ${ }^{2}$ anticipé à la retraite ; elles ont été exclues des statistiques, leur choix étant irréversible.

La figure 1 compare l'âge ${ }^{3}$ moyen des enseignants de l'enseignement obligatoire (y compris le maternel) en 1992 et en 2006. Durant cette période, on constate que dans le réseau libre, l'âge moyen a progressé de 2 ans au niveau fondamental ordinaire, de 3 ans au niveau secondaire ordinaire et a diminué de 1 an dans l'enseignement spécialisé. Dans le réseau de la Communauté française, l'âge moyen a baissé de 3 années dans le fondamental ordinaire et de 1 an dans le secondaire ordinaire et le spécialisé. Un vieillissement de 4 ans du personnel enseignant est observé au niveau secondaire ordinaire communal ainsi que dans l'enseignement spécialisé provincial. En 2006, tous réseaux confondus, l'âge moyen est de 39 ans au niveau fondamental ordinaire, de 43 ans au niveau secondaire ordinaire et de 41 ans dans l'enseignement spécialisé. De 1992 à 2006, seul l'âge moyen du niveau secondaire ordinaire a augmenté (+ 2 ans).

La figure 2 représente l'évolution de la distribution par âge du personnel enseignant de 1992 à $2006^{4}$ et met en évidence la tendance au vieillissement. En 1992, la moitié des enseignants considérés ont plus de 40 ans et, à partir de 1996 jusque 2006, ils ont plus de 41 ans. Cette particularité se retrouve dans les différents niveaux et plus spécifiquement dans l'enseignement secondaire ordinaire qui a connu une période d'engagements massifs de jeunes enseignants vers la fin des années 70 et le début des années 80 . Cette période se traduit par un pic de fréquences très importantes dans la distribution par âge. Cette affluence d'enseignants résulte de l'augmentation des effectifs scolaires combinée aux réformes structurelles engendrées par l'enseignement rénové à cette époque. Le fait que ce pic se déplace au fil des années confirme l'idée que le vieillissement des enseignants est la conséquence de l'évolution au cours du temps de notre système scolaire. De même, le creux observé dans la tranche des 35 à 42 ans en 2006 peut notamment s'expliquer par trois réformes introduites au début des années 90 . Celles-ci ont affecté le mode de calcul du nombre de charges enseignantes, ont engendré la fusion d'écoles et ont conduit à une diminution des redoublements au premier degré. Autant de paramètres qui, associés à la chute des effectifs des élèves du secondaire apparue simultanément, n'ont fait que freiner les recrutements.

La figure 3 représente la déperdition du nombre d'enseignants observée par niveau d'enseignement à partir de 55 ans, âge officiel du départ à la retraite anticipée. Un taux de déperdition a été calculé successivement pour chaque âge (à partir de 55 ans), en comparant les effectifs en activité âgés de " $x$ " années en 2005 avec les effectifs encore en activité âgés de " $x+1$ » années en 2006. Le fait d'appliquer le taux de déperdition observé à 55 ans à une base de 100 enseignants de 54 ans revient à illustrer le pourcentage d'enseignants qui décident de bénéficier des mesures de fin de carrière à partir de 55 ans $^{5}$. Puis, si on applique le taux de déperdition observé à 56 ans au nombre d'enseignants de 55 ans encore en activité ainsi déduit, on obtient le nombre d'enseignants qui choisissent de mettre fin à leur carrière à 56 ans. En procédant de la sorte pour les années suivantes, on peut déduire que la plupart des enseignants optent pour une retraite anticipée entre 55 et 60 ans. Ces derniers représentent respectivement $91 \%, 82 \%$ et $81 \%$ aux niveaux fondamental, secondaire et spécialisé. Une grande majorité le fait avant 59 ans dans le fondamental contre un peu plus de la moitié dans le secondaire et le spécialisé. Ces disparités s'expliquent notamment par le nombre de femmes plus important au niveau fondamental (cf indicateur 32).

En moyenne, dans les dix ans à venir, environ 2600 enseignants atteindront l'âge de 55 ans chaque année (fond : 800, sec : 1600 et spécialisé : 200) et pourront opter pour un départ anticipé dans le cadre des mesures d'aménagement de fin de carrière, à condition que celles-ci soient reconduites au-delà de l'année scolaire 2008-2009. L'indicateur 29 montre que 3967 personnes ${ }^{6}$ ayant suivi des formations pédagogiques de niveau supérieur de plein exercice ont été diplômées en 2005.

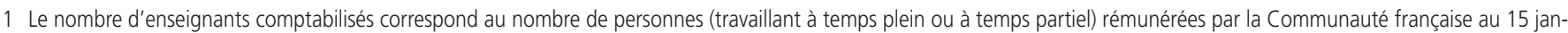
vier de l'année scolaire. II prend en compte non seulement les enseignants en poste mais également ceux qui sont détachés, en mission, en congé de maternité, de maladie, etc.

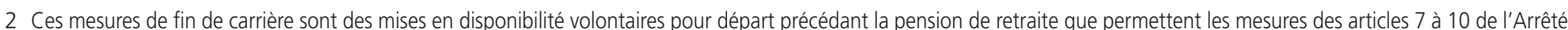
Royal du 31 mars 1984.

3 L'âge considéré est celui atteint au 31 décembre 2006 pour l'année scolaire 2005-2006, soit, par exemple, 40 ans pour les personnes nées en 1966.

4 Les années de référence disponibles avant 2004 sont 1992, 1996 et 2002. Elles correspondent aux années de publication des annuaires statistiques.

5 Sur le graphique, ils se retrouvent principalement à 56 ans car la collecte s'établit au 15-01-2006 et l'âge considéré est celui atteint au 31-12-2006.

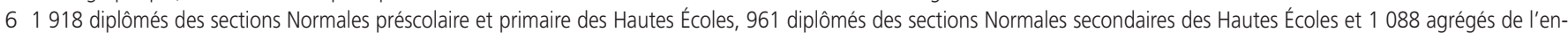
seignement secondaire supérieur. 


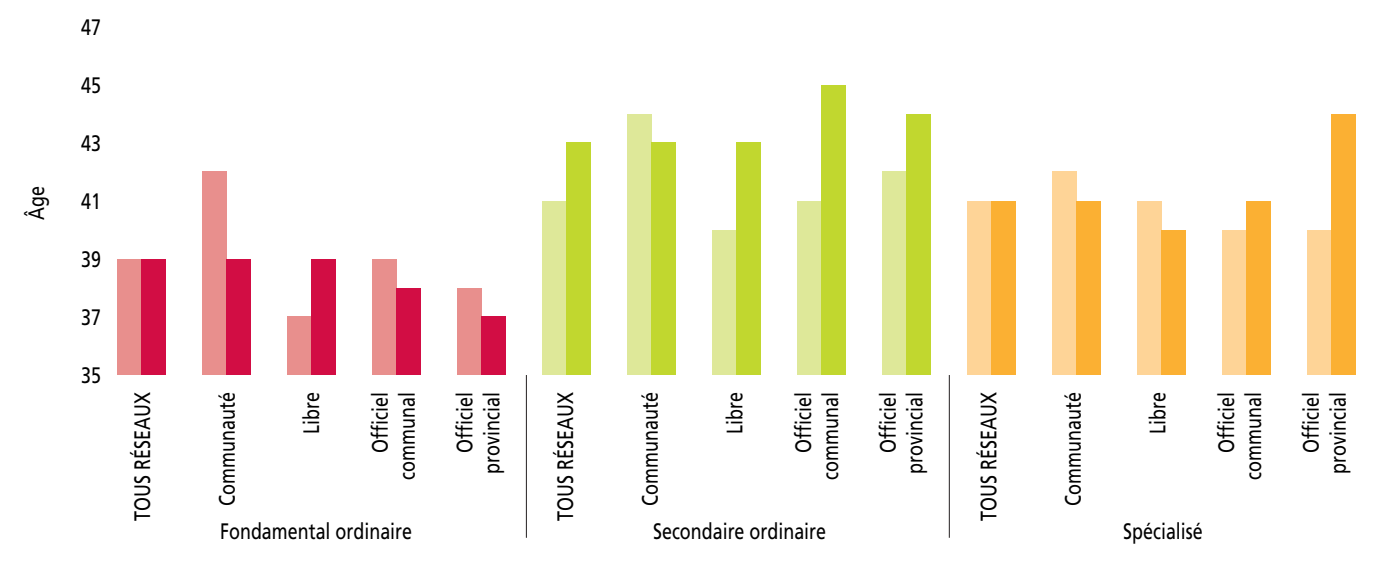

—1991-1992

2005-2006

Dans l'enseignement fondamental ordinaire, l'âge moyen du personnel enseignant de la Communauté est de 42 ans en 1992 et de 39 ans en 2006.

31.2 Distribution par âge du personnel enseignant de l'enseignement obligatoire - Années 1992 à 2006

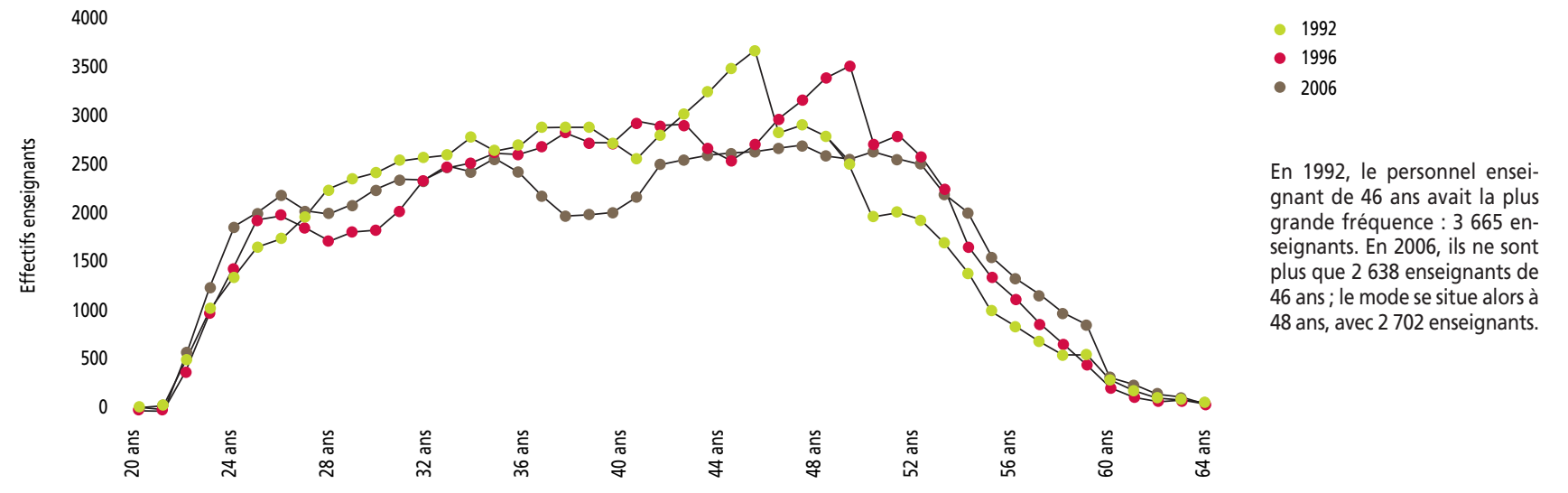

31.3 Déperdition du nombre d'enseignants à partir de 54 ans en base 100 - Année 2006

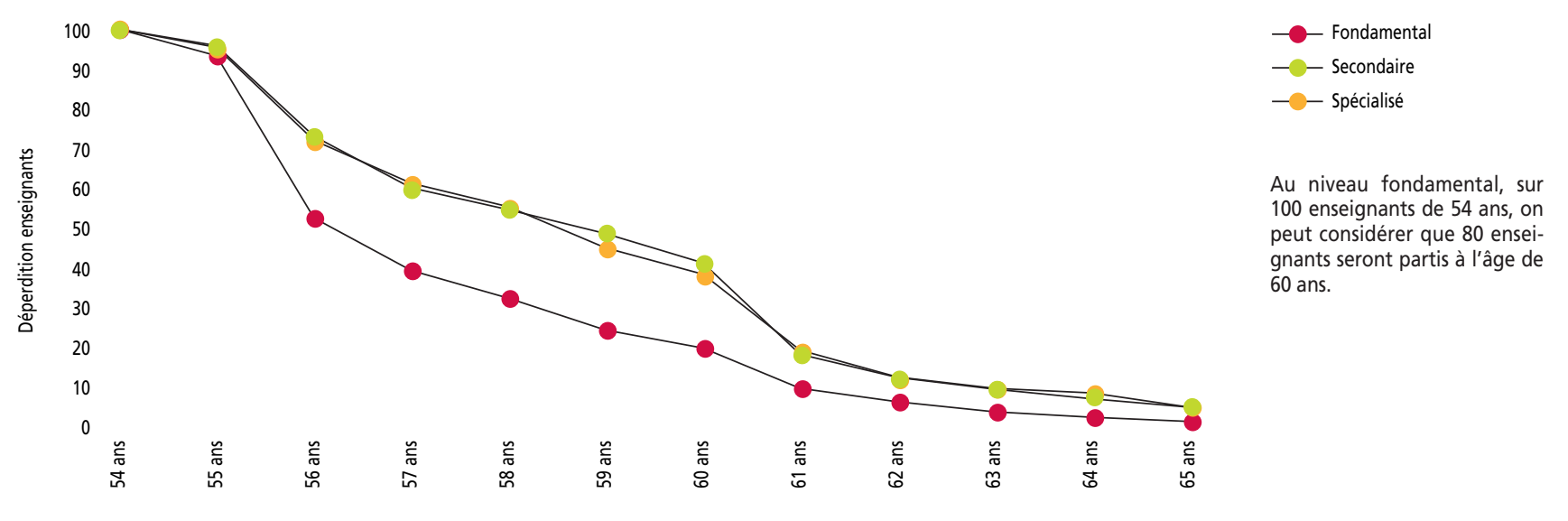


En 2006, dans l'enseignement fondamental ordinaire, plus de $85 \%$ des instituteurs sont des femmes ; dans l'enseignement secondaire ordinaire et dans l'enseignement spécialisé, elles représentent plus de $60 \%$ des enseignants.

La proportion de femmes diminue avec l'âge. Elles deviennent minoritaires à partir de 61 ans.

Au fil des années, l'enseignement se féminise de plus en plus.

e personnel de l'enseignement pris en considération correspond à

Ll'ensemble des enseignants rémunérés par la Communauté française, que la charge d'enseignement soit prestée ou non ${ }^{1}$, à l'exception des personnes ayant opté pour un départ ${ }^{2}$ anticipé à la retraite ; elles ont été exclues des statistiques, leur choix étant irréversible.

La figure 1 présente l'évolution de la proportion de femmes dans l'enseignement fondamental et secondaire ordinaire ainsi que dans l'enseignement spécialisé, de 1992 à 2006. II apparaît clairement que le taux de féminisation progresse, et ce, dans les mêmes proportions quel que soit le niveau ou la forme d'enseignement considéré.

L'enseignement fondamental ordinaire est le plus féminisé ; en 20052006 , la proportion de femmes y est de plus de $85 \%$. Ce taux se situe à $60 \%$ dans l'enseignement secondaire ordinaire et à près de $66 \%$ dans l'enseignement spécialisé.

Face à ces résultats, il semble que si l'enseignement est un choix professionnel principalement féminin, ce phénomène s'est renforcé au cours des dernières années. C'est également le cas de nombreux autres systèmes scolaires européens.

La figure 2 représente l'évolution du taux de féminisation selon l'âge du personnel enseignant de 1992 à $2006^{3}$. Elle permet de visualiser, d'une part, l'évolution de la proportion de femmes au fil de la carrière et, d'autre part, l'ampleur du phénomène dans le temps.

Elle montre tout d'abord que, l'âge avançant, la proportion de femmes décroît et, à partir de 55 ans, de manière significative. En 20052006, la diminution du taux de féminisation s'articule autour de trois moments charnières : de $89 \%$ à 21 ans, il n'est plus que de $59 \%$ à 55 ans et chute à $27 \%$ à 65 ans. Le premier moment se caractérise par le fait que le taux de féminisation le plus important concerne les femmes les plus jeunes. Ceci s'explique principalement par le fait que les femmes sont diplômées plus jeunes que les hommes et qu'elles enseignent majoritairement dans l'enseignement fondamental ordinaire qui nécessite un cursus initial moins long que pour l'enseignement secondaire supérieur. Le deuxième moment est lié au fait qu'à 55 ans, la proportion des enseignantes est affectée par les mesures de fin de carrière. En effet, de 55 à 60 ans, elles sont presque $15 \%$ de plus que les hommes à opter pour un départ anticipé à la retraite. Le troisième moment montre qu'après 60 ans, ce constat s'accentue puisque les femmes ont davantage tendance à partir à la retraite dès l'âge de 60 ans.

La figure 2 montre également que de 1992 à 2006, le taux de féminisation a progressé à tous les âges, à l'exception de la tranche des moins de 25 ans dans laquelle la proportion de femmes a régressé d'environ $5 \%$.

Les raisons qui peuvent expliquer cette évolution sont multiples. On évoquera notamment le fait que le métier permet de travailler à temps partiel et donc d'aider les femmes à concilier travail et famille. En effet, le nombre d'enseignants travaillant à temps partiel a considérablement augmenté depuis 1992, tout particulièrement dans l'enseignement fondamental ordinaire où l'on observe une croissance de l'ordre de $30 \%$, provoquée principalement par des femmes. La féminisation de l'enseignement n'est cependant pas la seule cause de cette augmentation. Le phénomène découle également de facteurs tels que l'évolution des charges : la difficulté de constituer des horaires complets dans un établissement produit une offre d'enseignement à temps partiel. Ainsi, le choix professionnel des hommes ne semble pas se porter aussi aisément que celui des femmes vers le secteur de l'enseignement. Le temps partiel ne constituant généralement qu'un salaire d'appoint, les hommes pourraient avoir tendance à s'orienter davantage vers d'autres horizons plus lucratifs et plus porteurs.

En conclusion, la fonction enseignante se caractérise par une féminisation croissante de l'enseignement et une réduction du temps de travail qui l'accompagne.

1 Le nombre d'enseignants comptabilisés correspond au nombre de personnes (travaillant à temps plein ou à temps partiel) rémunérées par la Communauté française au 15 janvier de l'année scolaire. Il prend en compte non seulement les enseignants en poste mais également ceux qui sont détachés, en mission, en congé de maternité, de maladie, etc.

2 Ces mesures de fin de carrière sont des mises en disponibilité volontaires pour départ précédant la pension de retraite que permettent les mesures des articles 7 à 10 de l'Arrêté Royal du 31 mars 1984.

3 Les années de référence choisies avant 2004 sont 1992, 1996 et 2002 parce qu'elles correspondent aux années de publication des annuaires statistiques. 
100

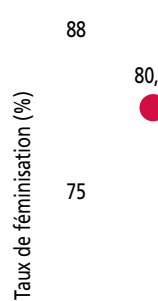

63
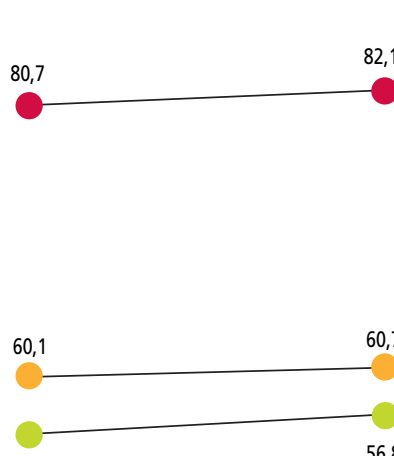

55,6
82,1

84,0

-
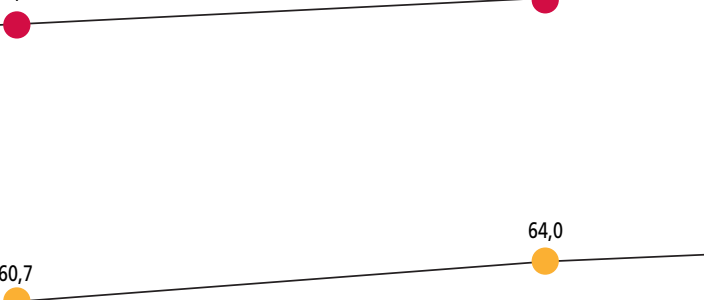

58,7
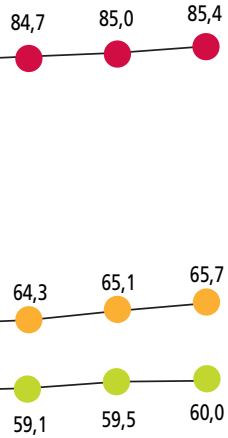

50

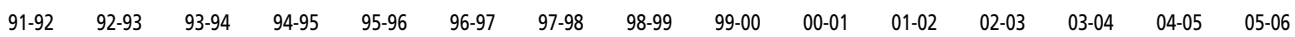

- fondamental ord

- spécialisé

- - secondaire ordin

En 2005-2006, le nombre d'inscriptions dans la section normale primaire est de 5809 étudiants alors que ce nombre était de 4090 étudiants en 1992-1993 et 5447 étudiants en 19951996.

\subsection{Distribution par âges du taux de féminisation du personnel enseignant de l'enseignement obligatoire - Années 1992 à 2006}

100

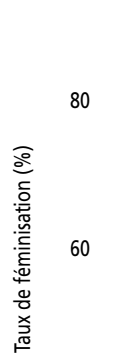

40

20

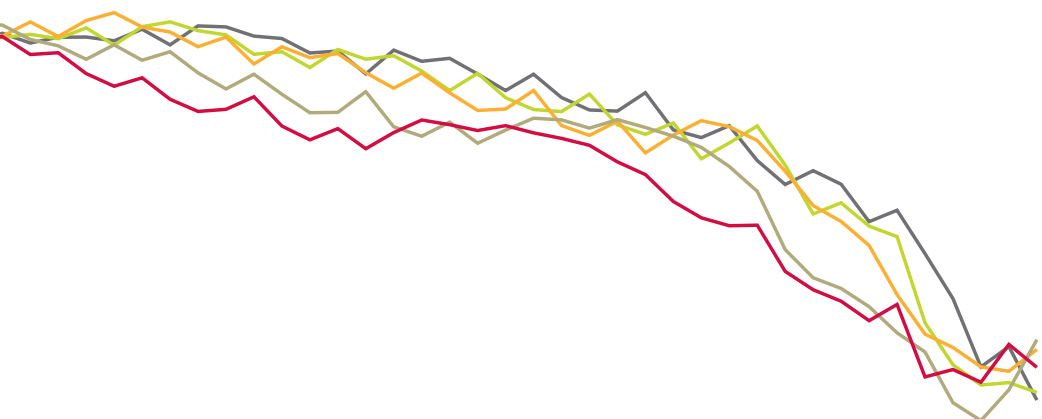

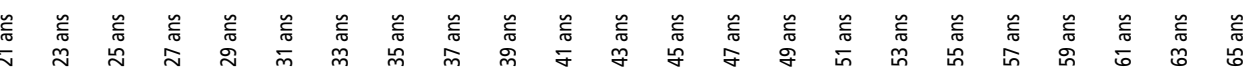

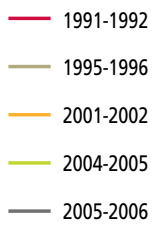

En fin d'année 20042005 , le nombre de diplômes d'instituteurs primaires délivrés est de 1285 , contre 807 en 1992-1993 ; les perspectives pour l'année académique 2007-2008 prévoient 1360 diplômes d'instituteurs primaires délivrés. 


\section{Sources}

\section{SOURCES COMMUNES}

Concernant l'enseignement maternel, primaire, secondaire et supérieur non universitaire en Communauté française.

Données statistiques portant sur les années scolaires 1998-1999 à 1993-1994

Service des Statistiques. Annuaires statistiques, Bruxelles: Ministère de l'Éducation, de la Recherche et de la Formation, Communauté française de Belgique.

Données statistiques portant sur les années scolaires 1994-1995 à 2003-2004

Service général de l'informatique et des Statistiques. Annuaires statistiques, Bruxelles: Ministère de la Communauté française de Belgique.

Données statistiques portant sur les années scolaires 1996-1997 et suivantes

AGERS (DGEO et DGENORS) et ETNIC, Service des Statistiques

http://www.statistiques.cfwb.be/

\section{SOURCES SUPPLEMENTAIRES PAR INDICATEUR}

\section{INDICATEUR 1}

Institut national de Statistique, Statistiques démographiques, Bruxelles, Ministère des Affaires économiques, Direction générale Statistique et Information économique.

OCDÉ (1997 à 2005). Regards sur l'Éducation, Les indicateurs de I'OCDÉ, OCDÉ - CERI, Paris : OCDÉ.

\section{INDICATEUR 2}

Institut national de Statistique, Statistiques démographiques, Bruxelles, Ministère des Affaires économiques, Direction générale Statistique et Information économique.

Conseil des recteurs francophones.

\section{INDICATEUR 3}

Ministère de la Communauté française (1997 à 2005). Regroupement économique des dépenses de la Communauté française.

\section{INDICATEUR 4}

Institut national de Statistique (2001). Perspectives de population 2000-2050, Bruxelles, Ministère des Affaires économiques, Direction générale Statistique et Information économique.

\section{INDICATEUR 7}

Demeuse, M. (2000). La politique de discrimination positive en Communauté française de Belgique: une méthode d'attribution des moyens supplémentaires basée sur des indicateurs objectifs. Les Cahiers du Service de Pédagogie expérimentale, 1-2, 115-135

(http://www.ulg.ac.be/pedaexpe/cahiers.html)

\section{INDICATEUR 19}

Conseil des recteurs francophones. 


\section{INDICATEUR 20}

Ministère de la Communauté française, Administration générale de l'Enseignement et de la Recherche scientifique, Service général du Pilotage du Système éducatif (2007). Évaluation externe non certificative. Lecture et production d'écrit. $2^{\mathrm{e}}$ année de l'enseignement primaire. Résultats et commentaires. Bruxelles: Auteur.

\section{INDICATEUR 21}

Ministère de la Communauté française, Administration générale de l'Enseignement et de la Recherche scientifique, Service général du Pilotage du Système éducatif (2007). Évaluation externe non certificative. Lecture et production d'écrit. $5^{e}$ année de l'enseignement primaire. Résultats et commentaires. Bruxelles: Auteur.

\section{INDICATEUR 22}

Ministère de la Communauté française, Administration générale de l'Enseignement et de la Recherche scientifique, Service général du Pilotage du Système éducatif (2007). Évaluation externe non certificative. Lecture et production d'écrit. $2^{\mathrm{e}}$ année de I'enseignement secondaire. Résultats et commentaires. Bruxelles: Auteur.

\section{INDICATEUR 23}

OCDÉ (2007a). Les compétences en sciences, un atout pour réussir. Volume 1 : Analyses des résultats. Paris : Auteur.

OCDÉ (2007b). Les compétences en sciences, un atout pour réussir. Volume 2 : Données. Paris : Auteur.

\section{INDICATEUR 24}

Droesbeke J.-J., Hecquet I., Wattelar C. (2001). La population étudiante. Description, évolution, perspectives. Bruxelles : ULB.

\section{INDICATEUR 28}

Conseil des recteurs francophones.

\section{INDICATEUR 25}

Beckers J., Jaspar S. et Voos M.-C. (2003). Attirer, former et retenir des enseignants de qualité. Rapport présenté par la Communauté française dans le cadre de l'étude thématique de l'OCDE.

\section{INDICATEURS 30, 31 ET 32}

ETNIC, Service des Statistiques, fichiers historiques de la cellule de calcul des traitements.

Indicateurs, sources et annexes complémentaires à consulter sur le site : www.enseignement.be 
Cette série d'indicateurs de l'enseignement n'aurait pu être réalisée sans la collaboration de nombreuses personnes : les auteurs, les membres du groupe du projet, les relecteurs ainsi que les membres de la Commission de Pilotage du système éducatif.

\section{AUTEURS DES INDICATEURS}

Abdelkrim ABDELKHALED, Service des Statistiques, ETNIC

Ariane BAYE, Unité d'Analyse des Systèmes et des Pratiques d'Enseignement de I'Université de Liège Violaine DEFOURNY, Service des Statistiques, ETNIC Philippe DIEU, Service des Statistiques, ETNIC Jean-Marie DUPIERREUX, Service des Statistiques, ETNIC

Béatrice GHAYE, Service général du Pilotage du système éducatif

Catherine LEFEVRE, Service des Statistiques, ETNIC Lamia MECHBAL, Service général du Pilotage du système éducatif

\section{MEMBRES DU GROUPE DU PROJET}

Emmanuel de PATOUL, Service " Développement ", ETNIC

Carine D'HAMERS, Service « Développement ", ETNIC

Jean-Marie DUPIERREUX, Service des Statistiques, ETNIC

Béatrice GHAYE, Service général du Pilotage du système éducatif

Martine HERPHELIN, Service général du Pilotage du système éducatif

Julien NICAISE, Cabinet de la Ministre-Présidente Yves VANDEBOSSCHE, Service général du Pilotage du système éducatif

\section{RELECTEURS}

André CAUSSIN, Inspection de l'enseignement spécialisé

Pol COLLIGNON, Inspection de l'enseignement fondamental

Louis DELARGE, Service général du Pilotage du système éducatif

Sébastien DELATTRE, Service général du Pilotage du système éducatif

Michel GEORIS, Service général du Pilotage du système éducatif

Lise-Anne HANSE, Direction générale de

I'enseignement obligatoire

Nathalie JAUNIAUX, Direction des relations internationales

Arlette VANDERKELEN, Inspection de

I'enseignement fondamental

Georges VANLOUBBEECK, Inspection de

I'enseignement secondaire

Anne WILMOT, Service général du Pilotage du système éducatif

Léon ZAKS, Direction générale des affaires générales et d'audit budgétaire et financier 